\title{
Modulating Bond Lengths via Backdonation: A First- Principles Investigation of a Quinonoid Zwitterion Adsorbed to Coinage Metal Surfaces
}

\section{SUPPORTING INFORMATION}

Scott Simpson, James Hooper, Daniel P. Miller, Donna A. Kunkel, Axel Enders, and Eva Zurek

S1. ZI DOS

S2. Further Comments on the Validity of the Molecular Clusters.

S3. Bond lengths: Comparison between the experimental and optimized lattice constant....................S7

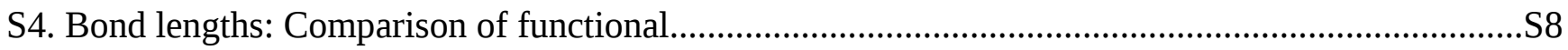

S5. Partial Charge Density Isovalue Plots of the ZI frontier orbitals on the metal surfaces.

S6. Bond Lengths of ZI with Fractional Occupations.

S7. Alternative Networks.

S8. Binding site analysis of p-benzoquinonemonoimine on $\mathrm{Cu}(111), \mathrm{Ag}(111)$, and $\mathrm{Au}(111) \ldots . . . \mathrm{S} 15-\mathrm{S} 18$

S9. Change in the Workfunction upon Adsorption of ZI to Cu(111), Ag(111), and Au(111).... S19

S10. Nucleus Independent Chemical Shifts. S20

S11.Bond Strengthening of Benzoquinone. S21

S12. SI References .S22

S13. Coordinates. S23-S133 
$\underline{\text { S1. ZI DOS }}$

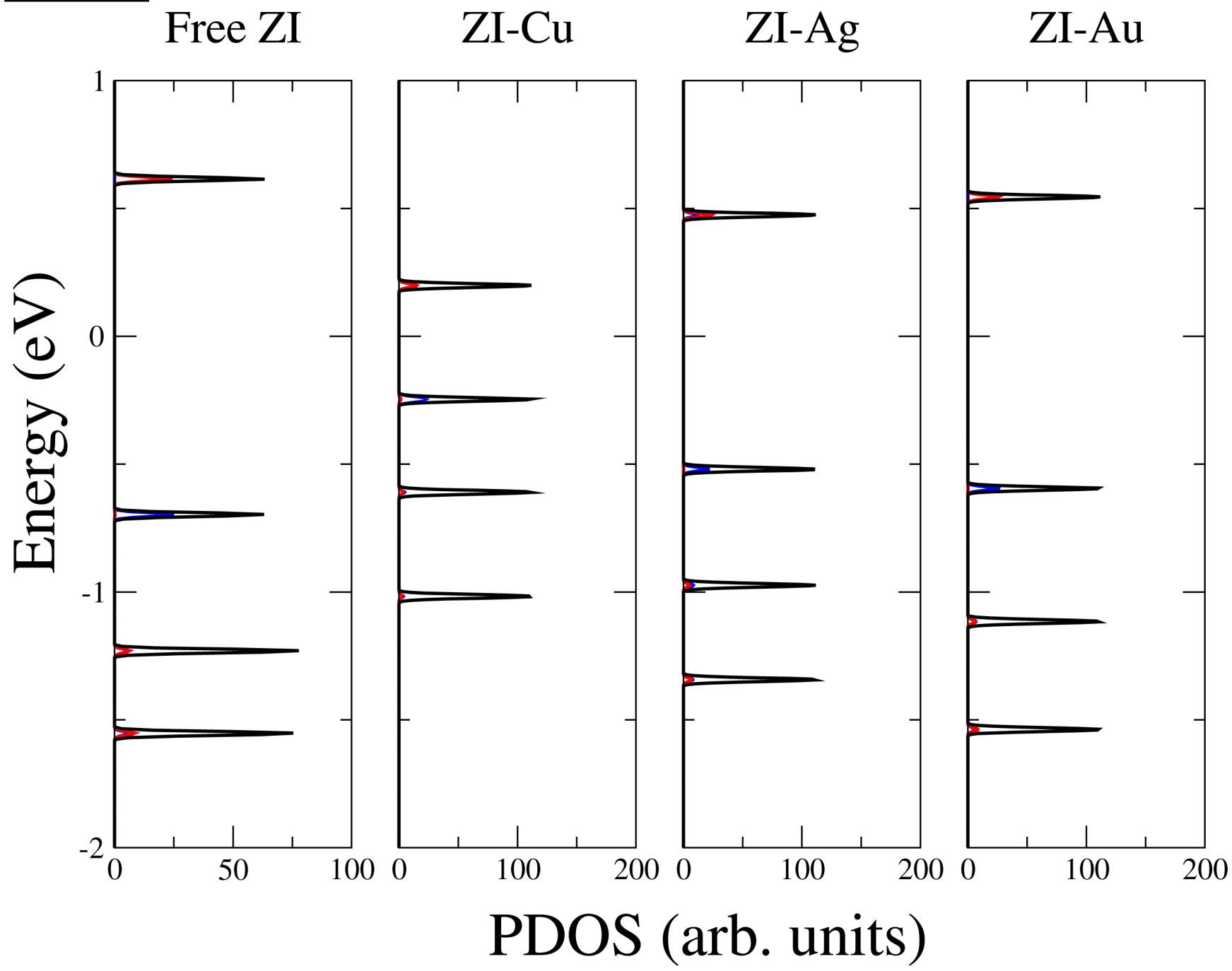

Figure S1: Projected Density of States (PDOS) of an isolated ZI molecule (Free ZI) and the ZI in the conformation it adopts when adsorbed to $\mathrm{Cu}, \mathrm{Ag}$, and Au as calculated in VASP with revPBE-D3. Atoms that contribute only to the HOMO are projected in blue while atoms that contribute only to the LUMO are projected in red.

The geometry of the ZI influences the band-gap of the molecule. The free isolated molecule has a band gap of approximately $1.30 \mathrm{eV}$ (left of Figure S1). When the molecule adopts the geometry that it assumes when adsorbed to the $\mathrm{Cu}(111)$ surface, the band gap decreases to approximately $0.40 \mathrm{eV}$. However this band-gap increases when adsorbed to the $\mathrm{Ag}(111)$ and $\mathrm{Au}(111)$ surface because the structural distortion is smaller. 


\section{$\underline{\text { S2. Further Comments on the Validity of the Molecular Clusters }}$}

Decomposed Binding Energies of 114-Atom Metal Cluster Models

In Table S1, we show the contributions to the binding energy that are presented graphically in Figure 5b of the main text.

\begin{tabular}{|l|l|l|l|l|l|l|}
\hline System & $\Delta$ Ebind & $\Delta$ Egeo & $\Delta$ Esteric & $\Delta$ Eoi & $\Delta$ Edisp & $\Delta$ EBSSE \\
\hline $\mathrm{Cu}(111)$ & -2.25 & 1.00 & 7.93 & -8.14 & -3.04 & -1.96 \\
\hline $\mathrm{Ag}(111)$ & -1.46 & 0.21 & 2.46 & -2.08 & -2.05 & -1.35 \\
\hline $\mathrm{Au}(111)$ & -1.91 & 0.17 & 2.34 & -2.11 & -2.31 & -1.78 \\
\hline
\end{tabular}

TABLE S1: Bond decompositions for molecular models in the main text.

We comment now on how the assignment of each NOCV interaction energy was assigned as either charge transfer into ZI or charge transfer from ZI. This was done by looking at the fragment orbital analysis that is performed when ETS-NOCV is run with the ADF program. If the largest contribution from the ZI molecule was an unoccupied fragment orbital, then the NOCV was assigned as being a slab $\rightarrow$ ZI charge transfer type; if the largest contribution from the ZI molecule was an occupied fragment orbital, then the NOCV was assigned as being a $\mathrm{ZI} \rightarrow$ slab charge transfer type. If the largest contributions to the NOCV from ZI comprised both occupied and unoccupied ZI fragment orbitals and the estimated change in the electron populations that accompanied the fragment orbitals were within $25 \%$, then the NOCV was classified as both (ie. colored yellow in Figure 5c in the main text). 


\section{Qualitative Pictures of Charge Transfer To and From the ZI}

In Figure 5 of the main text, we show how ETS-NOCV can be used to decompose the orbital interaction energy into contributions which transfer electron density to and from the adsorbed ZI molecule. Below, we show a qualitative representation of the two contributions. The shape of the charge density difference isosurfaces resembles charge transfer into the ZI LUMO (top plot, for charge transfer to ZI) and charge transfer from the ZI HOMO (bottom plot, for charge transfer from the ZI). The larger degree of charge density loss in the $\Delta \rho_{\mathrm{ZI} \rightarrow \text { slab }}$ isosurface around the periphery of the adsorbed ZI molecule especially highlights how the role of the other $\pi$-symmetry occupied molecular orbitals cannot be ignored and contribute substantially (as mentioned in the main text).
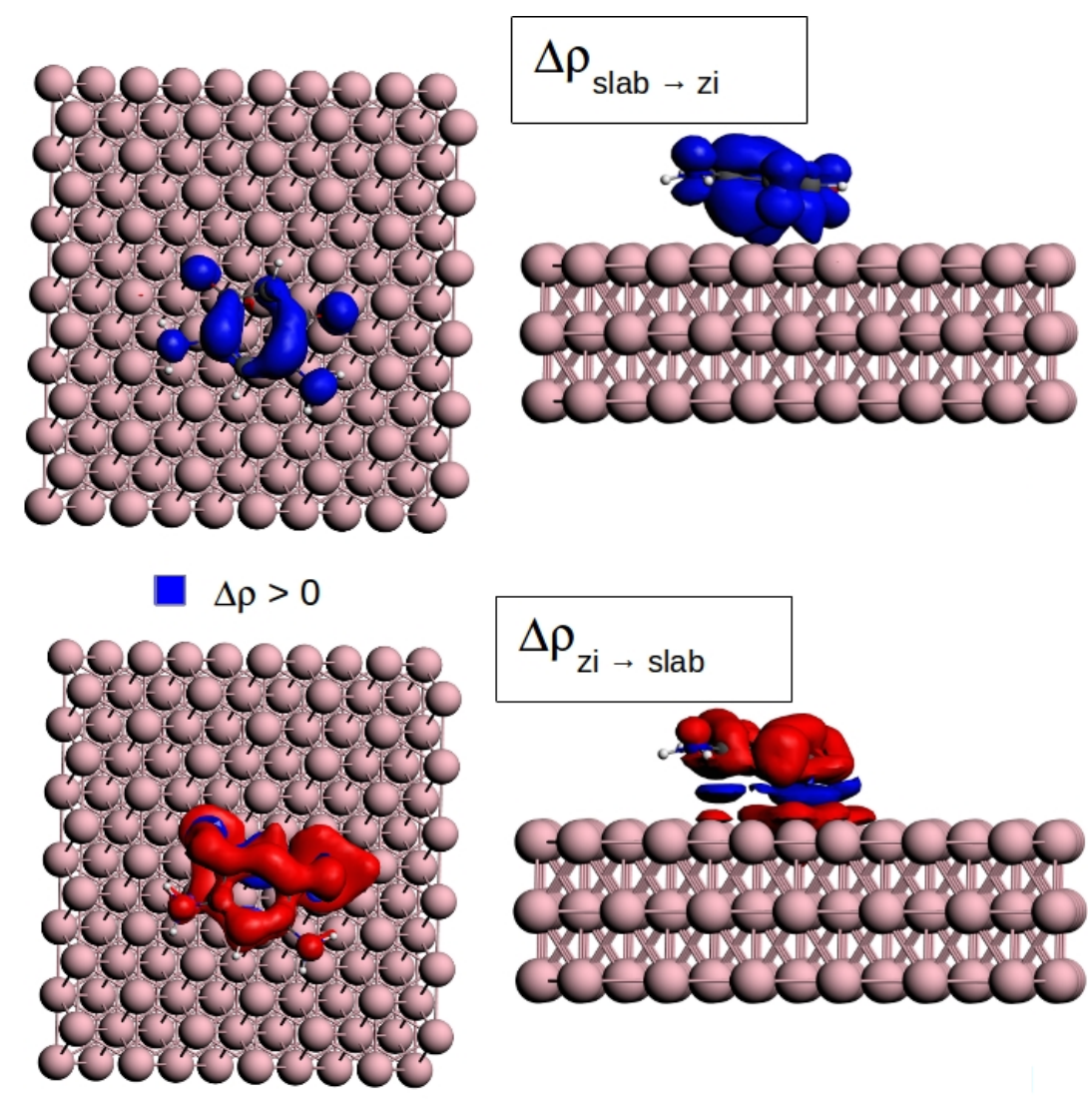

Figure S2: (top) An isosurface of the sum of the charge density difference densities that are generated from ETS-NOCV orbital pairs which populate fragment molecular orbitals on the ZI. (bottom) An isosurface of the sum of the charge density differences that are generated from ETS-NOCV orbital pairs which de-populate fragment molecular orbitals on the ZI. The charge density difference, $\Delta \rho$, corresponds to the difference between the electronic charge density of the total system and the sum of the electronic charge densities in the isolated fragments (ie. $\Delta \rho=\rho$ (system) $-\rho$ (ZI) $-\rho$ (cluster)). Blue represents an increase in electron density and red represents a decrease in electron density relative to the fragments. 


\section{Alternative Cluster Models}

The decomposition of the molecular revPBE-D3 interactions energies that are shown in the main text were obtained from cluster models that were extracted directly from the periodic models which were optimized with VASP. In Table S2, we show the decompositions of the binding energies which are obtained from finite-cluster models which fully relax the geometry of the ZI. These cluster models use 3-layer metal clusters which are made up of 130 atoms and are built such that the distance between $\mathrm{Cu}$ atoms corresponds with the experimental lattice constant of bulk $\mathrm{Cu}$.

\begin{tabular}{|l|l|l|l|l|l|l|l|}
\hline System & $\Delta$ EBSSE & $\Delta$ Emol & $\Delta$ Egeo & $\Delta$ Esteric & $\Delta$ Eoi & dsurf-ads & QH \\
\hline $\mathrm{Cu}(111)$ & -2.01 & -2.27 & 0.32 & 3.71 & -3.58 & 2.458 & +0.07 \\
\hline $\mathrm{Ag}(111)$ & -1.52 & -1.64 & 0.13 & 1.71 & -1.45 & 2.849 & +0.04 \\
\hline $\mathrm{Au}(111)$ & -1.72 & -1.87 & 0.09 & 1.89 & -1.53 & 2.800 & +0.25 \\
\hline $\mathrm{Cu}(111)^{\mathrm{a}}$ & -1.93 & -2.24 & 1.05 & 6.30 & -6.76 & 2.377 & -0.11 \\
\hline
\end{tabular}

${ }^{a}$ the ZI is optimized under the constraint that the $\sigma_{\mathrm{v}}$ symmetry of the system is preserved

TABLE S2: Bond decompositions for alternative molecular models.

The general trends between the Cu vs. Ag vs. Au binding energies, and their components, correlates with the models that are discussed in the main text. The most striking difference is the lower magnitude of the $\Delta \mathrm{E}_{\text {steric }}, \Delta \mathrm{E}_{\text {geo }}$, and $\Delta \mathrm{E}_{\text {oi }}$ terms in the relaxed Cu model, and this relates to the $\mathrm{ZI}$ molecule moving further away from the "surface" and distorting less (relative to the optimal gas-phase geometry) than it does in the periodic models. The contraction of the $2 \mathrm{C}$ - $\mathrm{C}$ bond that is seen with the relaxed $\mathrm{Cu}(111)$ cluster, $\sim 1.49 \AA$, is only half of the contraction that was seen from the periodic calculations. To better relate the cluster calculations to the binding site from the periodic calculations, the $\mathrm{Cu}(111)$ model can also be optimized with a symmetry constraint that preserves the $\sigma_{v}$ symmetry of the combined $\mathrm{ZI} / \mathrm{Cu}(111)$ system. This symmetry-constrained system is also shown in Table S2 and results in, for example, a 2C-C distance of $\sim 1.45 \AA$ that relates well with the periodic models.

The sensitivity of the 2C-C bond distance was also checked with respect to a number of cluster models where the ZI molecule is freely relaxed. The results are shown in Table S3. As was noted for the models discussed in the main text, the 2C-C bond becomes shorter in going from $\mathrm{Cu}$ to $\mathrm{Ag}$ to $\mathrm{Au}$, and the shorter 2C-C bond correlates with a higher LUMO population. Regarding the sensitivity of the ETS-NOCV decomposition with respect to the size of the finite cluster, we did note that the values were similarly as sensitive as the 2C-C distance and estimated LUMO populations from Table S3 are. The total orbital interaction energy, $\Delta \mathrm{E}_{\mathrm{o}}$, computed from the fully relaxed models varies substantially, for example, from $-2.64 \mathrm{eV}$ for the 104 -atom cluster to $-3.58 \mathrm{eV}$ for the 130 -atom cluster. The NOCV pair with the largest eigenvalue in both clusters is still, however, one which is best characterized as charge transfer from the slab into the ZI LUMO and the interaction energy of this pair is found to make up roughly $20 \%$ of the total $\Delta \mathrm{E}_{\mathrm{oi}}$ in both cases. The larger $\Delta \mathrm{E}_{\mathrm{oi}}$ values generally correlate with the shortest 2C-C bonds. The simplest explanation for the variation in $\Delta \mathrm{E}_{\mathrm{oi}}$ is that the distribution of discrete molecular orbitals around the clusters' HOMO-LUMO gaps give rise to states with different energies in the vicinity of the gap. It follows that the clusters with the highest-energy occupied fragment orbitals (the 130-atom and 64-atom models) have electronic states closer in energy to the ZI 
LUMO and, thereby, allow more back-donation into the ZI LUMO and a higher $\Delta \mathrm{E}_{\mathrm{oi}}$.

\begin{tabular}{|c|c|c|c|}
\hline System & $\sigma_{\mathrm{v}}$ symmetry? & 2C-C distance (A) & $\begin{array}{l}\text { LUMO population } \\
\text { (from ETS-NOCV) }\end{array}$ \\
\hline $\mathrm{Cu}, 130$ atoms, 3 layers & no & $\sim 1.49$ & 0.71 \\
\hline Ag, 130 atoms, 3 layers & no & $\sim 1.52$ & - \\
\hline $\mathrm{Au}, 130$ atoms, 3 layers & no & $\sim 1.54$ & - \\
\hline $\mathrm{Cu}, 130$ atoms, 3 layers & yes & $\sim 1.44$ & 1.51 \\
\hline $\mathrm{Cu}, 166$ atoms, 2 layers & yes & $\sim 1.46$ & 1.20 \\
\hline $\mathrm{Cu}, 104$ atoms, 2 layers & no & $\sim 1.51$ & 0.39 \\
\hline $\mathrm{Cu}, 64$ atoms, 2 layers & no & $\sim 1.49$ & 0.78 \\
\hline Au, 64 atoms, 2 layers & no & $\sim 1.54$ & 0.07 \\
\hline
\end{tabular}

${ }^{a}$ the estimated population is taken from the change in LUMO "population" associated with the highest-energy NOCVs which are best characterized with charge transfer into the ZI LUMO.

TABLE S3: A table of the 2C-C distances found with various molecular models (the distances are rounded to the nearest 0.01 to draw particular attention to the full range of values). Also shown is the link between 2C-C and the estimated change in the LUMO "density" that accompanies the NOCVs which best describe charge transfer from the surface into the LUMO. 


\section{S3. Bond lengths: Comparison between the experimental and optimized lattice constant}

Tests were carried out to see how the bond length contraction changes while using the experimental lattice constant of $\mathrm{Cu}(111)$ compared to that of the optimized lattice constant (theoretical) using VASP and revPBE-D3. The change in bond lengths is reported in angstroms in the following table. The bond lengths of the adsorbed system are compared to the free gas phase ZI molecule, where a positive value indicates a bond elongation while a negative value denotes a bond shortening. The binding energies were calculated to be $-2.365 /-2.363 \mathrm{eV}$ for the experimental/theoretical lattice constant, respectively.

\begin{tabular}{c|c|c|}
\hline Bond & Experimental & Theoretical \\
\hline C-O & 0.0470 & 0.0542 \\
\hline C-N & 0.0899 & 0.0923 \\
\hline 1 C-C & 0.0327 & 0.0265 \\
\hline 2 C-C & -0.0917 & -0.0975 \\
\hline 3 C-C & 0.0171 & 0.0161 \\
\hline
\end{tabular}

Table S4: Changes in bond lengths of the ZI adsorbed to $\mathrm{Cu}(111)$ compared to the gas phase bond lengths. The values are reported in angstroms. 


\section{S4. Bond lengths: Comparison of functional}

This section contains the bond lengths of the ZI adsorbed to $\mathrm{Cu}(111)$ as calculated with PBE-D2, vdWDF2, and optB88-vdW. The binding sites that were considered were with respect to the center of the carbon ring of the p-benzoquinonemonoimine and copper atoms on the surface. The experimental lattice constant of the copper face-centered cubic cell were used in the construction of the slabs. The bottom two layers of the four layer slab were kept fixed while the top two layers were allowed to relax during geometry optimizations. The bond lengths of the isolated ZI are contained in the "Gas Phase" columns.

\begin{tabular}{|c|c|c|c|c|c|}
\hline Bond & H & H30 & T & T30 & Gas Phase \\
\hline C-O & 1.281 & 1.334 & 1.319 & 1.332 & 1.259 \\
\hline $\mathbf{C}-\mathbf{N}$ & 1.400 & 1.420 & 1.410 & 1.423 & 1.326 \\
\hline $\mathbf{1}$ C-C & 1.448 & 1.442 & 1.424 & 1.441 & 1.408 \\
\hline $\mathbf{2}$ C-C & 1.436 & 1.435 & 1.438 & 1.434 & 1.541 \\
\hline $\mathbf{3} \mathbf{C}-\mathbf{C}$ & 1.412 & 1.412 & 1.402 & 1.410 & 1.393 \\
\hline
\end{tabular}

Table S5: The bond lengths of the ZI adsorbed to $\mathrm{Cu}(111)$ as calculated with PBE-D2.

\begin{tabular}{|c|c|c|c|c|c|}
\hline Bond & H & H30 & T & T30 & Gas Phase \\
\hline C-O & 1.311 & 1.345 & 1.300 & 1.333 & 1.264 \\
\hline $\mathbf{C}-\mathbf{N}$ & 1.356 & 1.433 & 1.350 & 1.427 & 1.333 \\
\hline $\mathbf{1} \mathbf{C}-\mathbf{C}$ & 1.415 & 1.419 & 1.408 & 1.452 & 1.410 \\
\hline $\mathbf{2} \mathbf{C}-\mathbf{C}$ & 1.483 & 1.434 & 1.494 & 1.435 & 1.546 \\
\hline $\mathbf{3} \mathbf{C}-\mathbf{C}$ & 1.402 & 1.404 & 1.400 & 1.420 & 1.395 \\
\hline
\end{tabular}

Table S6: The bond lengths of the ZI adsorbed to $\mathrm{Cu}(111)$ as calculated with vdW-DF2.

\begin{tabular}{|c|c|c|c|c|c|}
\hline Bond & $\mathbf{H}$ & $\mathbf{H 3 0}$ & $\mathbf{T}$ & $\mathbf{T 3 0}$ & Gas Phase \\
\hline $\mathbf{C}-\mathbf{O}$ & 1.319 & 1.336 & 1.320 & 1.334 & 1.256 \\
\hline $\mathbf{C}-\mathbf{N}$ & 1.411 & 1.422 & 1.411 & 1.423 & 1.325 \\
\hline $\mathbf{1} \mathbf{C}-\mathbf{C}$ & 1.419 & 1.435 & 1.420 & 1.435 & 1.406 \\
\hline $\mathbf{2} \mathbf{C}-\mathbf{C}$ & 1.434 & 1.431 & 1.433 & 1.433 & 1.535 \\
\hline $\mathbf{3} \mathbf{C}-\mathbf{C}$ & 1.400 & 1.407 & 1.400 & 1.407 & 1.389 \\
\hline
\end{tabular}

Table S7: The bond lengths of the ZI adsorbed to $\mathrm{Cu}(111)$ as calculated with optB88-vdW. 


\section{S5. Partial Charge Density Isovalue Plots of the ZI frontier orbitals on the metal surfaces}

In order to confirm the identities of the molecular orbitals projected out by the PDOS in Figure 3 in the main text, we have calculated the partial charge densities in an energy window thought to span the ZI frontier orbitals hybridizing with the surface. These partial charge densities match well with the ZI frontier orbitals plotted in Figure 1 of the main text.

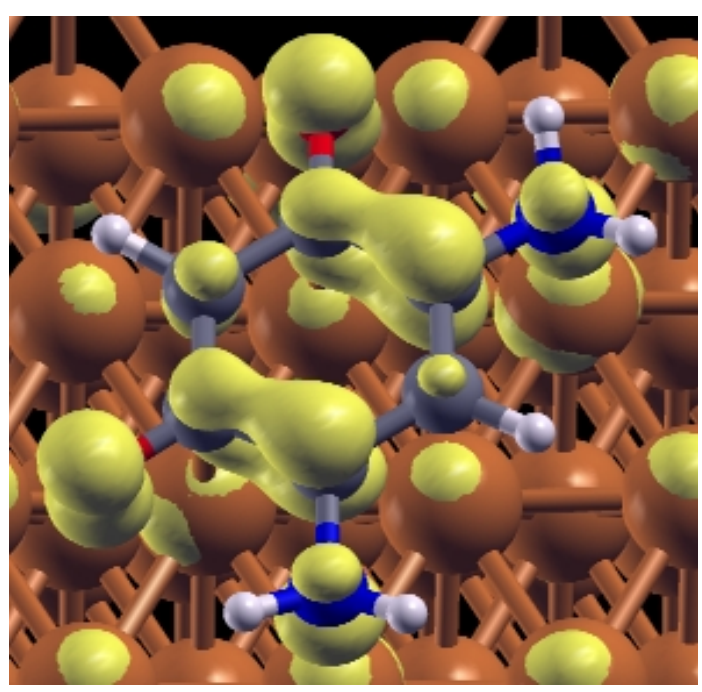

Figure S3: The partial charge density of the ZI adsorbed to $\mathrm{Cu}(111)$ over the energy interval of 0.00 to $-0.80 \mathrm{eV}$, with respect to the Fermi-level. This matches qualitatively with the ZI LUMO from our molecular calculations.

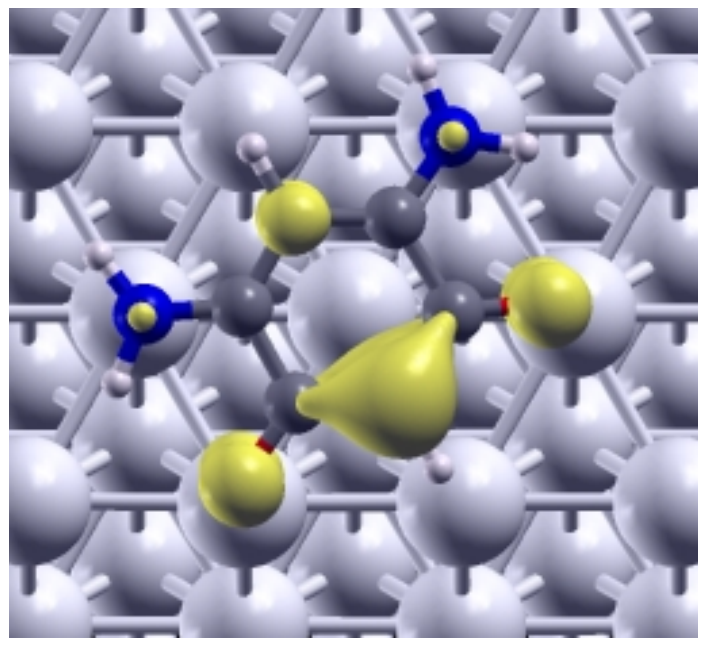

Figure S4: The partial charge density of the ZI adsorbed to Ag(111) over the energy interval of -1.00 to $-1.50 \mathrm{eV}$ with respect to the Fermi-level. This matches qualitatively with the ZI HOMO from our molecular calculations. 


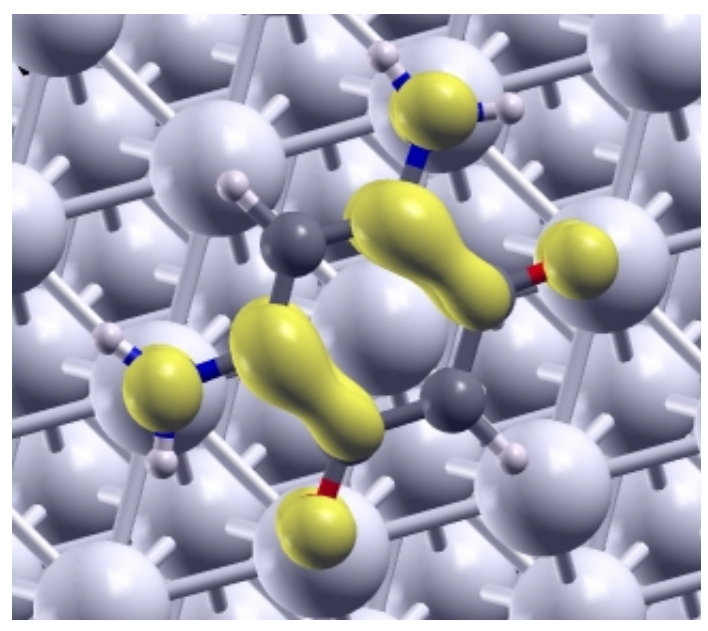

Figure S5: The partial charge density of the ZI adsorbed to Ag(111) over the energy interval of +0.50 to $-0.10 \mathrm{eV}$ with respect to the Fermi-level. This matches qualitatively with the ZI LUMO from our molecular calculations.

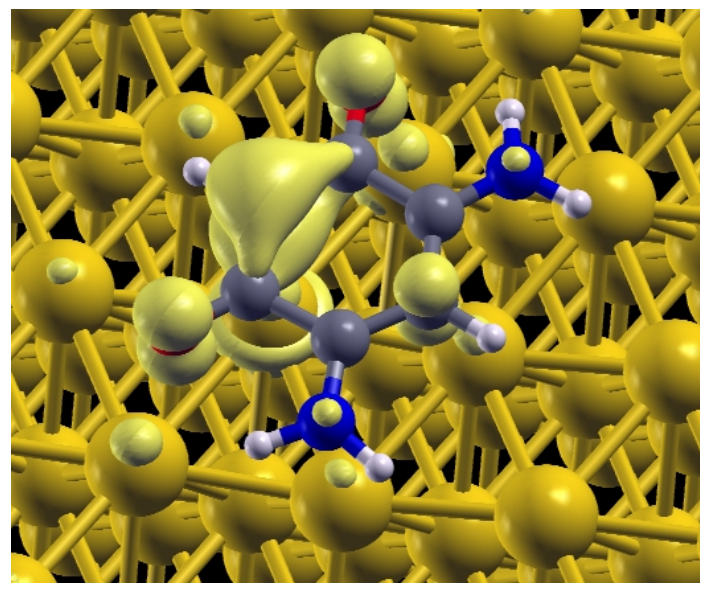

Figure S6: The partial charge density of the ZI adsorbed to Au(111) over the energy interval of -0.75 to $-1.20 \mathrm{eV}$ with respect to the Fermi-level. This matches qualitatively with the ZI HOMO from our molecular calculations. 


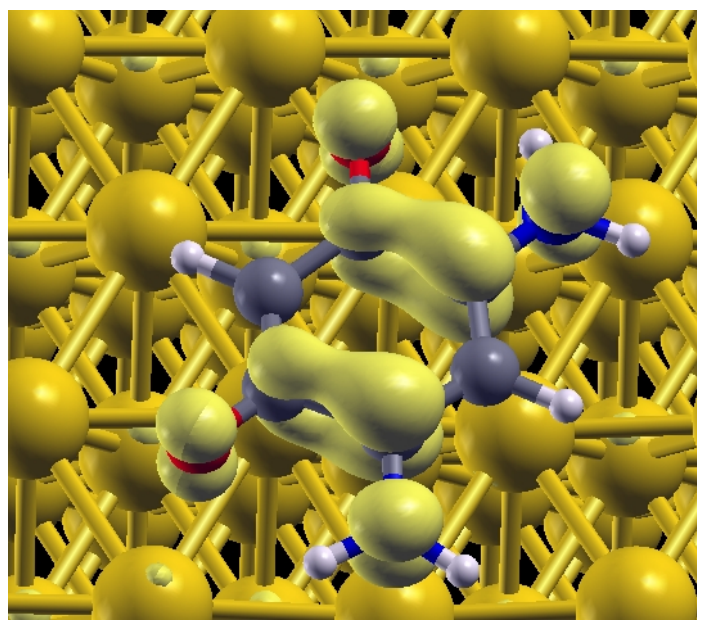

Figure S7: The partial charge density of the ZI adsorbed to Au(111) over the energy interval of +1.00 to $0.00 \mathrm{eV}$ with respect to the Fermi-level. This matches qualitatively with the ZI LUMO from our molecular calculations. 


\section{$\underline{\text { S6.Bond Lengths of ZI with Fractional Occupations }}$}

The bond lengths of the parent quinonoid zwitterion were calculated with fractional occupations using the ADF software package, revPBE-D3, and TZP basis set on all atoms (C/N/O had a 1 s core frozen). A HOMO occupation of 2 with a LUMO occupation of 0 corresponds to the geometry from an aufbau configuration. The bond lengths are reported in angstroms.

\begin{tabular}{c|c|c|c|c|c|c|}
\hline HOMO Occ. & LUMO Occ. & C-O & C-N & 1 C-C & 2 C-C & 3 C-C \\
\hline 2 & 0 & 1.261 & 1.331 & 1.411 & 1.552 & 1.397 \\
\hline 1.9 & 0.1 & 1.261 & 1.335 & 1.415 & 1.543 & 1.398 \\
\hline 1.8 & 0.2 & 1.262 & 1.339 & 1.418 & 1.534 & 1.399 \\
\hline 1.7 & 0.3 & 1.263 & 1.342 & 1.422 & 1.525 & 1.399 \\
\hline 1.6 & 0.4 & 1.264 & 1.346 & 1.426 & 1.516 & 1.400 \\
\hline 1.5 & 0.5 & 1.265 & 1.349 & 1.429 & 1.508 & 1.402 \\
\hline 1.4 & 0.6 & 1.266 & 1.352 & 1.432 & 1.500 & 1.403 \\
\hline 1.3 & 0.7 & 1.268 & 1.355 & 1.435 & 1.493 & 1.404 \\
\hline 1.2 & 0.8 & 1.269 & 1.357 & 1.438 & 1.485 & 1.405 \\
\hline 1.1 & 0.9 & 1.271 & 1.360 & 1.440 & 1.478 & 1.407 \\
\hline 1 & 1 & 1.273 & 1.363 & 1.442 & 1.472 & 1.408 \\
\hline 0.9 & 1.1 & 1.275 & 1.365 & 1.444 & 1.465 & 1.410 \\
\hline 0.8 & 1.2 & 1.277 & 1.368 & 1.446 & 1.459 & 1.411 \\
\hline 0.7 & 1.3 & 1.279 & 1.370 & 1.448 & 1.453 & 1.412 \\
\hline 0.6 & 1.4 & 1.281 & 1.372 & 1.449 & 1.447 & 1.414 \\
\hline 0.5 & 1.5 & 1.283 & 1.375 & 1.451 & 1.442 & 1.415 \\
\hline 0.4 & 1.6 & 1.286 & 1.377 & 1.452 & 1.437 & 1.416 \\
\hline 0.3 & 1.7 & 1.288 & 1.380 & 1.453 & 1.432 & 1.418 \\
\hline 0.2 & 1.8 & 1.291 & 1.382 & 1.453 & 1.427 & 1.419 \\
\hline 0.1 & 1.9 & 1.294 & 1.385 & 1.454 & 1.422 & 1.420 \\
\hline 0 & 2 & 1.296 & 1.387 & 1.455 & 1.417 & 1.421 \\
\hline
\end{tabular}

Table S8: The bond lengths of the ZI with fractional occupations. The values in the table are reported in angstroms.

The bond lengths in the ZI adsorbed to $\mathrm{Cu}(111)$ are actually not too different from the dianion in the gas phase. The bond lengths for the dianion of the ZI are: 1 C-C: $1.440,2$ C-C: $1.445,3$ C-C: 1.411, C-N: 1.431 and C-O: 1.333 angstroms. The Hirschfeld charges indicate that the electronic reorganization at the metal-organic interface results in a negative charge of $0.11 e$ on the ZI. We wondered if it would be possible to attain bond lengths in the gas phase neutral molecule that matched up with those observed in the ZI adsorbed to $\mathrm{Cu}(111)$ by simply changing the occupations of the frontier MOs? Towards this end, we optimized the geometry of the neutral gas phase ZI while keeping the occupations of the HOMO and the LUMO fixed. The resulting $\mathrm{C}-\mathrm{C}$ bond lengths are plotted in Figure S8. As the HOMO population is decreased and the LUMO increased, the $1 \mathrm{C}-\mathrm{C}$ and $3 \mathrm{C}-\mathrm{C}$ bonds elongate slightly. However, the most dramatic change is the pronounced contraction of the $2 \mathrm{C}-\mathrm{C}$ bond that occurs because an orbital that is pi-bonding across this bond is being populated. In fact, the $\mathrm{C}-\mathrm{C}$ distances within the ZI adsorbed on $\mathrm{Cu}(111)$ correlates with a HOMO population of 0.9-1.6 and a LUMO occupation of 0.6-1.1. This computational experiment illustrates that modifying the occupations of the frontier MOs in the neutral molecule can result in bond length changes similar to those observed 
upon adsorption to $\mathrm{Cu}(111)$. The slight negative charge we calculate of the $\mathrm{ZI}$ adsorbed to $\mathrm{Cu}(111)$ is consistent with its geometry.

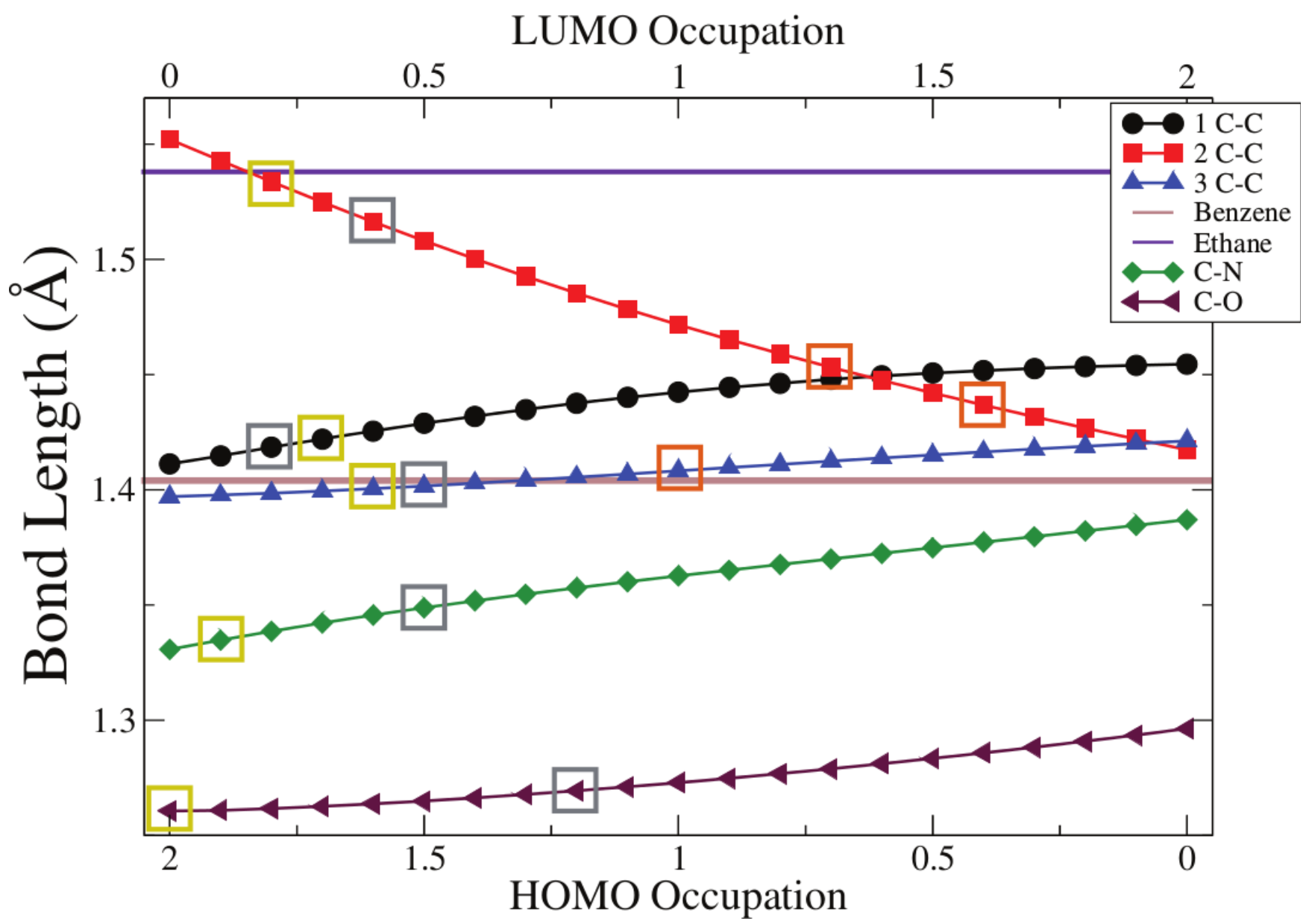

Figure S8: Bond distances of the neutral ZI in the gas phase as a function of the HOMO/LUMO occupation. The $\mathrm{C}-\mathrm{C}$ bond distances for ethane and benzene are indicated as straight lines on the plot. The orange/grey/yellow squares indicate the bond lengths of the ZI when adsorbed to copper/silver/gold, respectively. 


\section{$\underline{\text { S7.Alternative Networks }}$}

The following networks were constructed and optimized with revPBE-D3 in the VASP framework. These networks all show a pronounced bond shortening of the 2C-C bond.
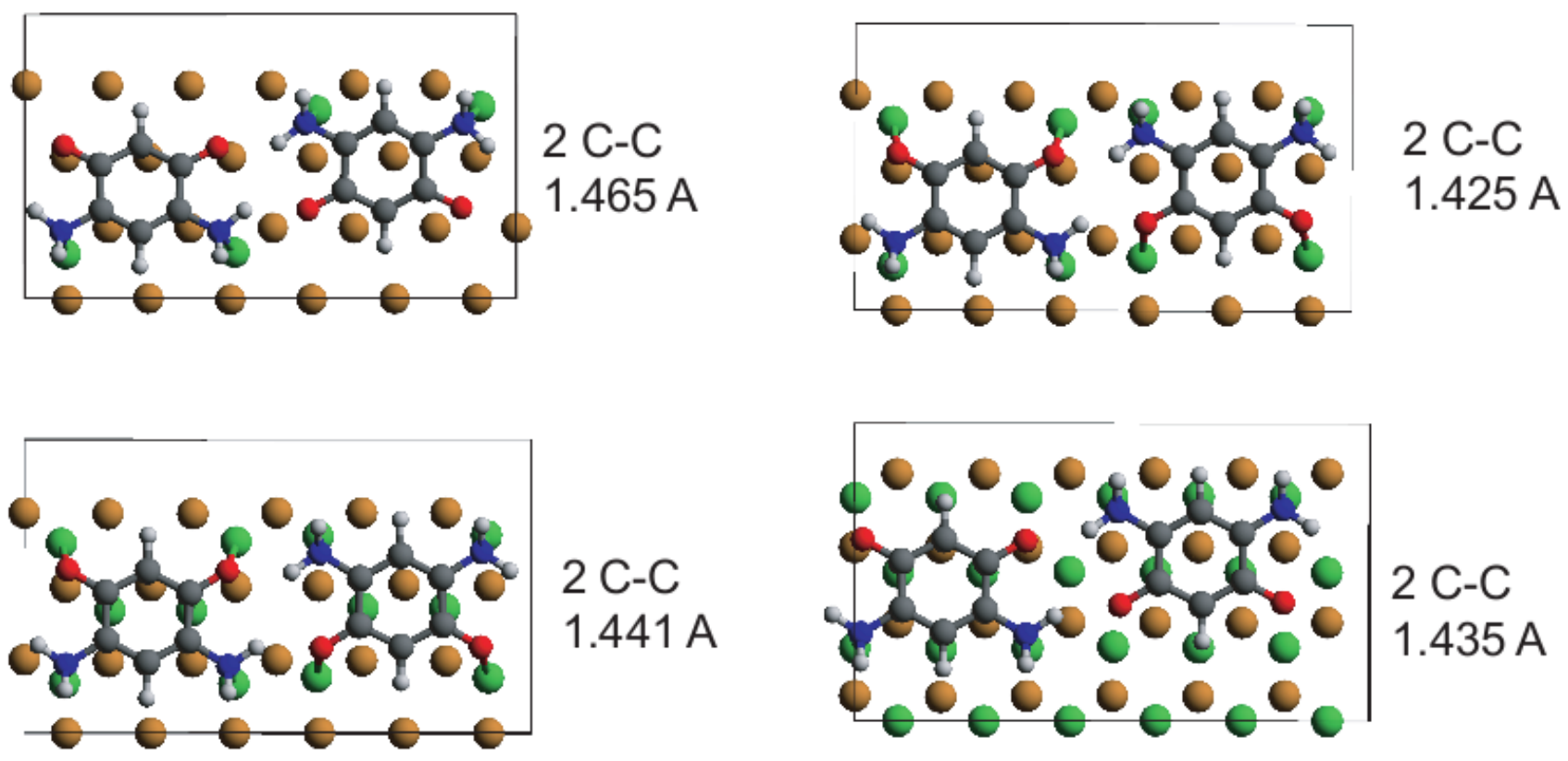

$2 \mathrm{C}-\mathrm{C}$

$1.435 \mathrm{~A}$

Figure S9: Alternative zipper-row networks. The copper atoms highlighted in green are ad-atoms which have been added to the original copper slab. 


\section{S8.Binding site analysis of p-benzoquinonemonoimine on $\mathrm{Cu}(111), \mathrm{Ag}(111)$, and $\mathrm{Au}(111)$}

To ascertain the preferred binding site for p-benzoquinonemonoimine on the $\mathrm{Cu}(111), \mathrm{Ag}(111)$, and $\mathrm{Au}(111)$ surfaces, the modeling method explained in SI Ref. 1 was adopted. Recently, we had investigated the potential energy landscape of these systems with RPBE-D3, rather than revPBE-D3 utilized in this work. [SI Ref. 2] From there, various binding sites were analyzed, as shown in Figure S9, through the manipulation of the adsorbate geometry with respect to the (111) surfaces. Figure S9 was constructed to display three rotational high-symmetry configurations $\left(0^{\circ}, 30^{\circ}, 60^{\circ}\right)$ possible for pbenzoquinonemonoimine to adsorb to when placed above a top $(\mathrm{T})$ site, hcp-hollow $\left(\mathrm{H}_{\mathrm{hcp}}\right)$ site, fcchollow $\left(\mathrm{H}_{\mathrm{fcc}}\right)$ site, or bridge $(\mathrm{B})$ site for the center of the ring. These three high-symmetry orientations above the four ring binding sites (BS) (12 total configurations for each surface) were considered in the binding site analysis.

The $\mathrm{Cu}(111), \mathrm{Ag}(111)$ and $\mathrm{Au}(111)$ surfaces were modeled with the computed lattice constant from revPBE-D3, the geometry was optimized with the revPBE-D3 functional, and IVDW was set to 11.
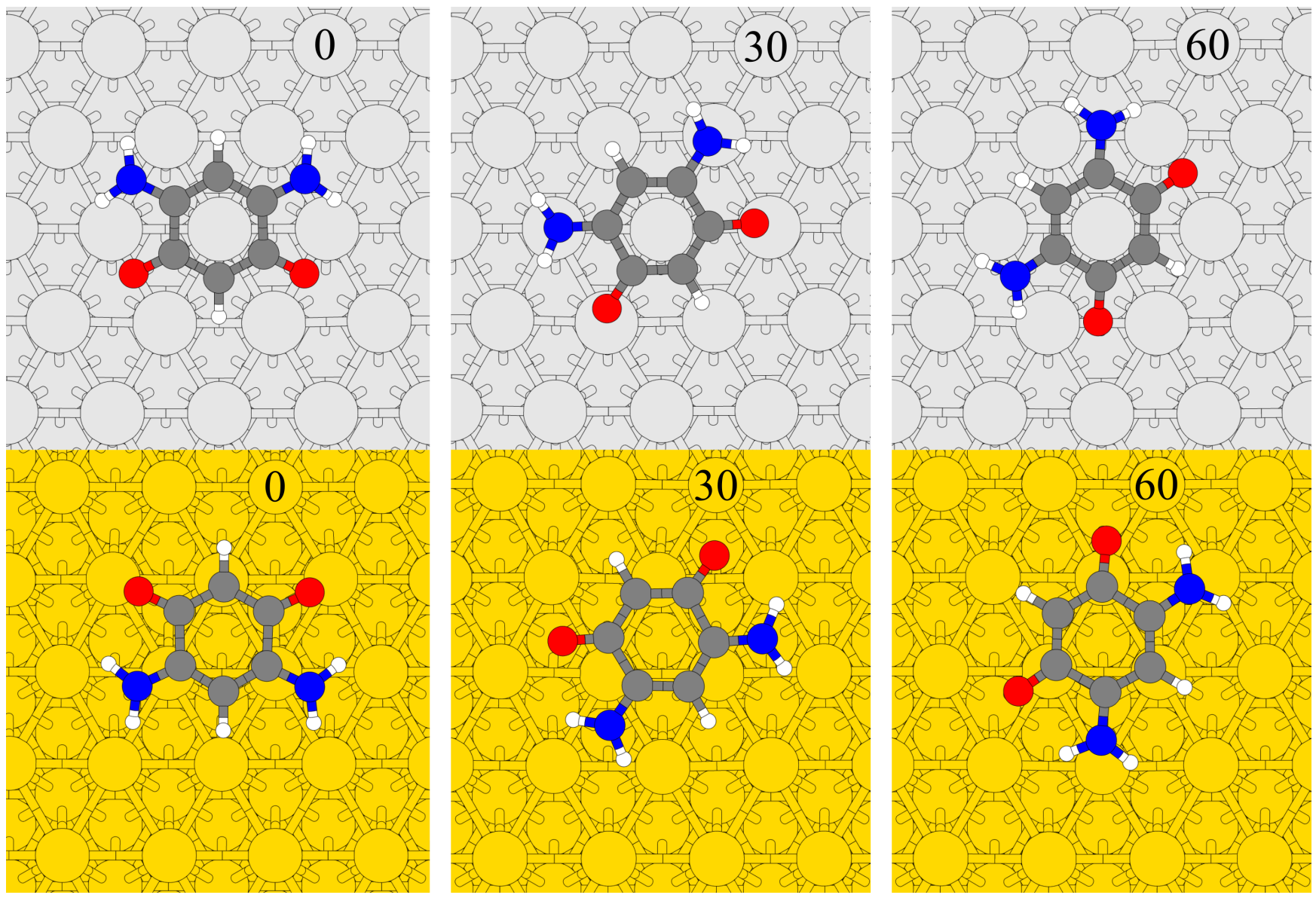

Figure S10: The $0^{\circ}, 30^{\circ}$, and $60^{\circ}$ orientations of p-benzoquinonemonoimine adsorbed to the $\mathrm{T}$ ring binding site of the $\mathrm{Ag}(111)$ surface (top-half) and to the $\mathrm{H}_{\mathrm{fcc}}$ ring binding site of the $\mathrm{Au}(111)$ surface (bottom-half) detailing the modeling method adopted from SI Ref. 1 for usage with the ZI. The hydrogen, carbon, oxygen, nitrogen, silver, and gold atoms are colored white, gray, red, blue, light gray, and yellow, respectively. 


\begin{tabular}{|c|c|c|c|}
\hline \multicolumn{4}{|c|}{$\mathrm{Cu}(111)$} \\
\hline BS & $\Delta E(\mathrm{eV})$ & BS & $\Delta E(e V)$ \\
\hline$T-0$ & 0.54 & Hhcp - 0 & $\mathbf{0}$ \\
\hline $\mathrm{T}-30$ & 0.11 & Hhcp - 30 & 0.43 \\
\hline $\mathrm{T}-60$ & 0.50 & Hhcp -60 & 0.22 \\
\hline $\mathrm{H}_{\mathrm{fcc}-\mathrm{O}} \mathrm{O}$ & 0.19 & $B-0$ & 0.05 \\
\hline $\mathrm{H}_{\mathrm{fcc}}-30$ & 0.35 & $\mathrm{~B}-30$ & 0.32 \\
\hline $\mathrm{H}_{\mathrm{fcc}}-60$ & 0.04 & $\mathrm{~B}-60$ & 0.16 \\
\hline
\end{tabular}

Table S9: BS (binding sites) and relative energies $(\Delta \mathrm{E})$ of the systems with respect to the most stable configuration for p-benzoquinonemonoimine adsorbed to the $\mathrm{Cu}(111)$ surface.

The preferred configuration is the $\mathrm{H}_{\mathrm{hcp}}-0$, as confirmed by SI Ref. 2 .

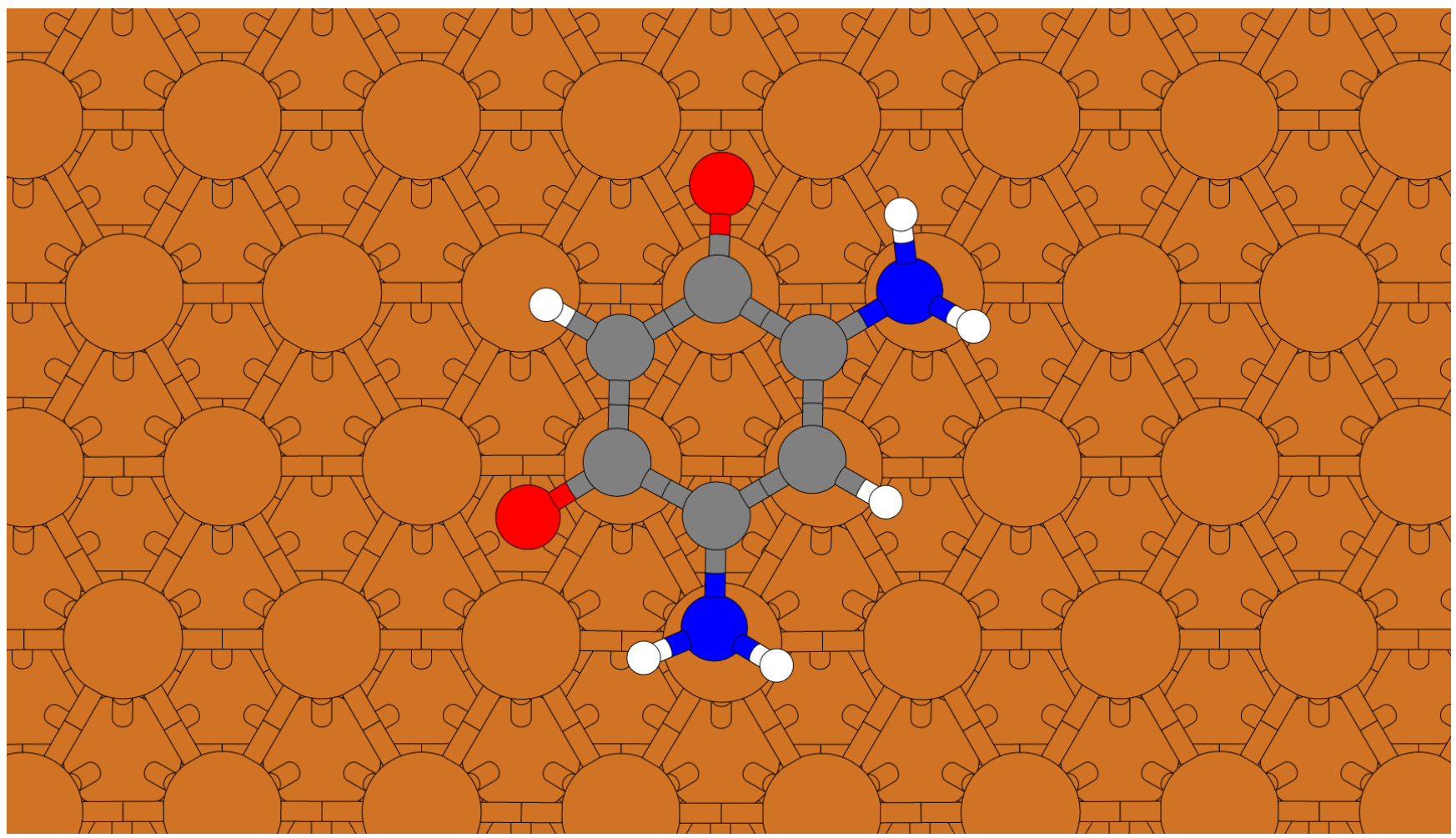

Figure S11: The most stable configuration $\left(\mathrm{H}_{\mathrm{hcp}}-0\right.$ from Table S9) of p-benzoquinonemonoimine adsorbed to the $\mathrm{Cu}(111)$ surface. The oxygens lie above the $\mathrm{H}_{\mathrm{fcc}} \mathrm{BS}$, the nitrogens lie above the $\mathrm{T} \mathrm{BS}$, and the center of the ring is above a $\mathrm{H}_{\text {hcp }}$ BS. The hydrogen, carbon, oxygen, nitrogen, and copper atoms are colored white, gray, red, blue, and orange, respectively. 


\begin{tabular}{|c|c|c|c|}
\hline \multicolumn{4}{|c|}{$\underline{\operatorname{Ag}(111)}$} \\
\hline BS & $\Delta E(\mathrm{eV})$ & BS & $\Delta E(\mathrm{eV})$ \\
\hline $\mathrm{T}-0$ & 0.11 & Hhcp - 0 & 0.14 \\
\hline$T-30$ & $\mathbf{0}$ & Hhcp - 30 & 0.01 \\
\hline $\mathrm{T}-60$ & 0.03 & Hhcp - 60 & 0.05 \\
\hline $\mathrm{H}_{\mathrm{fcc}-} 0$ & 0.06 & $B-0$ & 0.13 \\
\hline $\mathrm{H}_{\mathrm{fcc}-}-30$ & 0.04 & $B-30$ & 0.17 \\
\hline $\mathrm{H}_{\mathrm{fcc}-}-60$ & 0.10 & $B-60$ & 0.12 \\
\hline
\end{tabular}

Table S10: BS and relative energies $(\Delta \mathrm{E})$ of the systems with respect to the most stable configuration for p-benzoquinonemonoimine adsorbed to the $\mathrm{Ag}(111)$ surface.

The preferred configuration is the $\mathrm{T}-30$.

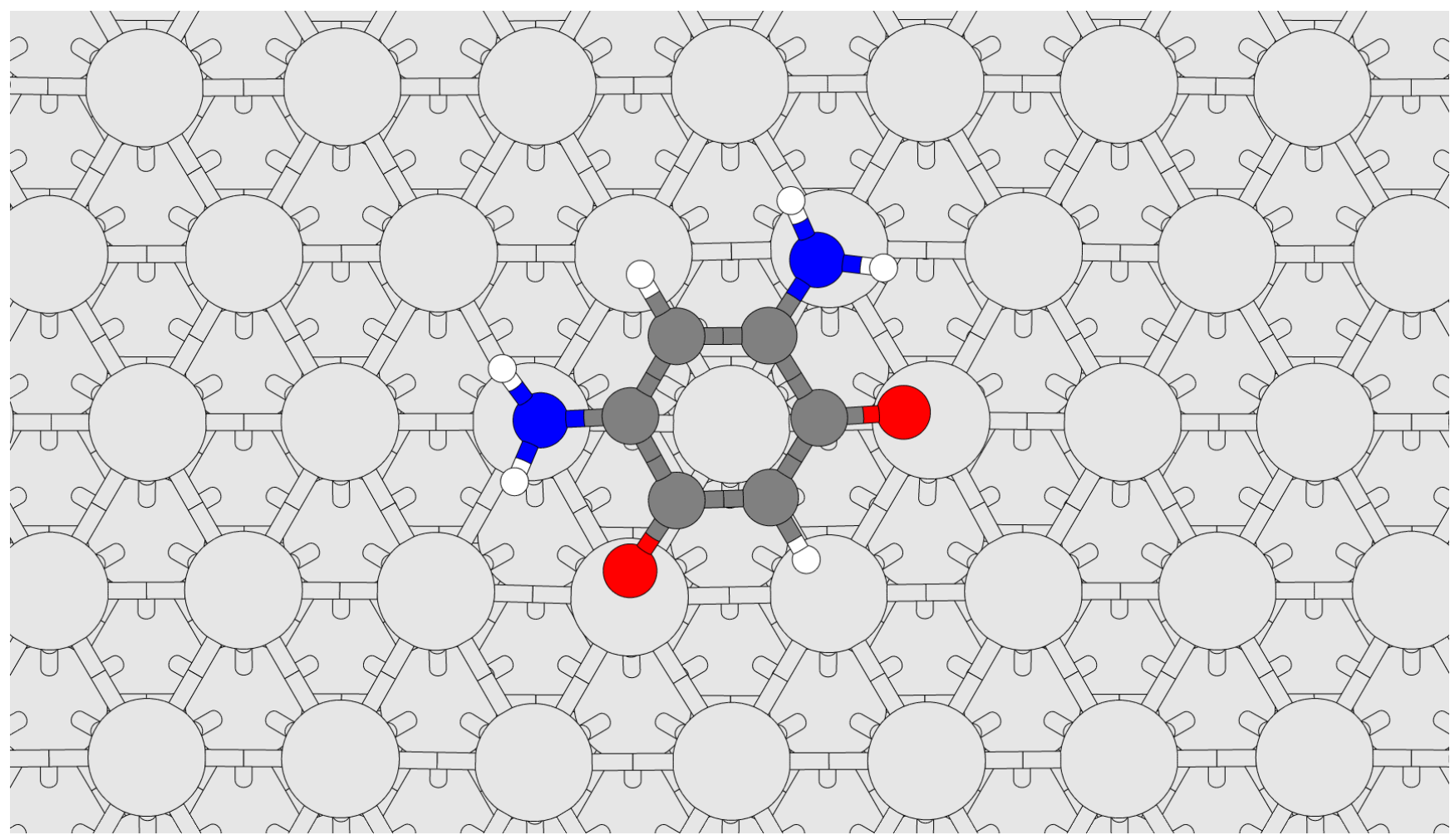

Figure S12: The most stable configuration ( $\mathrm{T}$ - 30 from Table S10) of p-benzoquinonemonoimine adsorbed to the Ag(111) surface. The nitrogens and oxygens both lie above T BS and the center of the ring is above a T BS. These results agree with the conclusions from SI Ref. 1 in regards to its nitrogen and oxygen atoms both being above T BS of the Ag(111) surface. The hydrogen, carbon, oxygen, nitrogen, and silver atoms are colored white, gray, red, blue, and light gray, respectively.

The main text draws its data from the structure displayed in Figure S12 for Ag(111). 


\begin{tabular}{|c|c|c|c|}
\hline \multicolumn{4}{|c|}{$\underline{\operatorname{Au}(111)}$} \\
\hline BS & $\Delta E(\mathrm{eV})$ & BS & $\Delta E(e V)$ \\
\hline $\mathrm{T}-0$ & 0.08 & Hhcp - 0 & 0.15 \\
\hline $\mathrm{T}-30$ & 0.09 & Hhcp - 30 & 0.02 \\
\hline $\mathrm{T}-60$ & 0.06 & Hhcp - 60 & 0 \\
\hline $\mathrm{H}_{\mathrm{fcc}-} 0$ & 0.03 & $B-0$ & 0.10 \\
\hline $\mathrm{H}_{\mathrm{fcc}}-30$ & 0.06 & $\mathrm{~B}-30$ & 0.11 \\
\hline $\mathrm{H}_{\mathrm{fcc}-}-60$ & 0.12 & B - 60 & 0.02 \\
\hline
\end{tabular}

Table S11: BS and relative energies $(\Delta \mathrm{E})$ of the systems with respect to the most stable configuration for p-benzoquinonemonoimine adsorbed to the $\mathrm{Au}(111)$ surface.

The preferred configuration is the $\mathrm{H}_{\mathrm{hcp}}-60$.

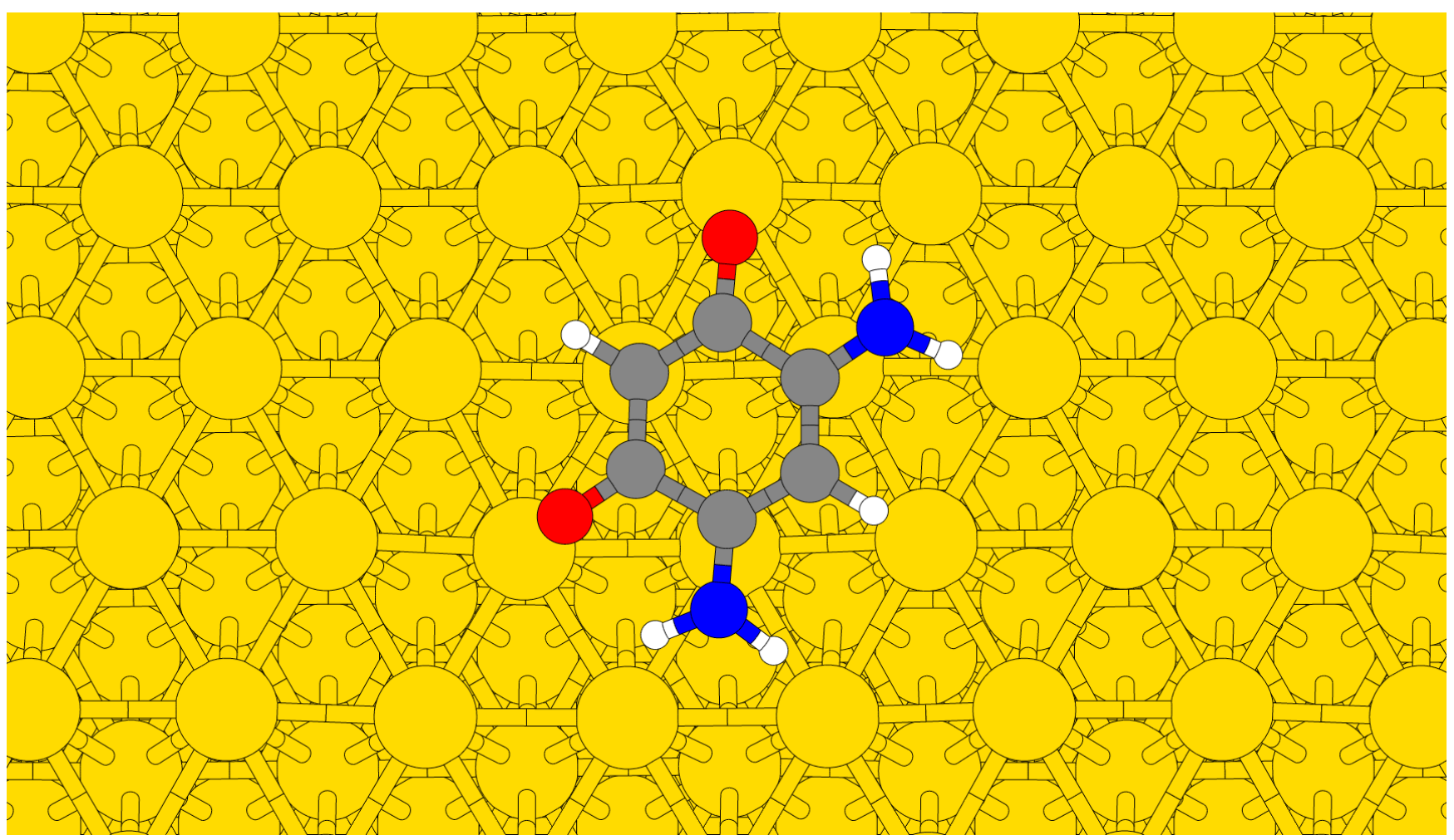

Figure S13: The most stable configuration $\left(\mathrm{H}_{\mathrm{hcp}}-60\right.$ from Table S11) of p-benzoquinonemonoimine adsorbed to the $\mathrm{Au}(111)$ surface. The oxygens lie above T BS, the nitrogens lie above $\mathrm{H}_{\mathrm{fcc}} \mathrm{BS}$, and the center of the ring is above a $\mathrm{H}_{\mathrm{hcp}} \mathrm{BS}$. The hydrogen, carbon, oxygen, nitrogen, and gold atoms are colored white, gray, red, blue, and yellow, respectively.

The main text draws its data from the structure displayed in Figure S13 for Au(111). 


\section{S9.Change in the Workfunction upon Adsorption of ZI to Cu(111), Ag(111), and Au(111)}

Through the LVHAR tag, as implemented in VASP, the change in workfunction ( $\Delta \Psi)$ upon adsorption of the ZI to the surface was calculated for $\mathrm{Cu}(111), \operatorname{Ag}(111)$, and $\mathrm{Au}(111)$. The values are as follows:

$\mathrm{ZI} / \mathrm{Cu}(111) \Delta \Psi:-0.46 \mathrm{eV}$

ZI/Ag(111) $\Delta \Psi:-0.17 \mathrm{eV}$

$\mathrm{ZI} / \mathrm{Au}(111) \Delta \Psi:-0.30 \mathrm{eV}$ 


\section{S10.Nucleus Independent Chemical Shifts}

Nucleus Independent Chemical Shifts (NICS) were calculated with the ADF software package, the revPBE functional with the Grimme3 empirical dispersion correction, and TZP basis set. The NICS $(0)$ is the NICS value calculated at the center of the ring. The NICS(0)zz is the zz tensor component at the center of the ring. The NICS(1) is the NICS value calculated at $1 \AA$ above the center of the ring. The NICS(1)zz is the zz tensor component at $1 \AA$ above the center of the ring. These values can be found for various systems below in Table S12.

\begin{tabular}{|c|c|c|c|c|c|c|c|c|}
\hline System & NICS(0) & Ref & NICS(1) & Ref & NICS(0)zz & Ref & NICS(1)zz & Ref \\
\hline ThioFuran & -12.6 & $-14.3^{\mathrm{c}}$ & -10.1 & $-11.5^{\mathrm{c}}$ & -9.6 & $-13.0^{\mathrm{c}}$ & -28.3 & $-30.4 \mathrm{C}$ \\
\hline Cyclobutadiene & 26.6 & $26.5^{\mathrm{b}}$ & 17.5 & $17.4 \mathrm{~d}^{\mathrm{d}}$ & 108.9 & $110.3^{\mathrm{b}}$ & 56.0 & $59.9^{\mathrm{a}}$ \\
\hline Cyclopentadiene-cation & 92.9 & $87.9^{\mathrm{b}}$ & 71.2 & - & 279.1 & $268.0^{\mathrm{b}}$ & 217.5 & $204.9^{\mathrm{a}}$ \\
\hline Cyclopentadiene-anion & -12.9 & $-12.6^{\mathrm{b}}$ & -10.1 & $-11.5^{\mathrm{c}}$ & -16.5 & $16.2^{\mathrm{b}}$ & -32.9 & $-33.7^{\mathrm{a}}$ \\
\hline Benzene & -7.5 & -7.9 & -9.6 & -10.1 & -13.3 & $-14.5^{\mathrm{b}}$ & -27.8 & $-29.2^{\mathrm{a}}$ \\
\hline ZI & 7.1 & - & 2.7 & - & 30.0 & - & 11.5 & - \\
\hline ZI-4Cu & -8.2 & - & -5.3 & - & -16.7 & - & -12.4 & - \\
\hline Dianion ZI & -9.1 & - & -7.0 & - & -1.3 & - & -15.0 & - \\
\hline
\end{tabular}

a Nancy S. Mills, N.S.; Llagostera, K.B. J. Org. Chem., 2007, 72, 9163-9169.

${ }^{\text {b }}$ Preze-Juste, I.; Mandado, M.; Carballeria, L. Chem. Phys. Lett., 2010, 491, 224-229.

c Fallah-Bagher-Shaidaei, H.; Wannere, C.S.; Corminboeuf, c.; Puchta, R.; Schleyer, P.R.Org.Lettt., 2006, 8 , 863-866.

d Zhi-Xiang Wang, Z.-X.; von Ragué Schleyer, P. Science, 2001, 292, 2465-2469.

Table S12: NICS(0), NICS(1), NICS(0)zz, NICS(1)zz values calculated for various systems along with reference values (Ref). 


\section{S11.Bond Strengthening of Benzoquinone}

We have found that the C1-C2 bond of ortho- benzoquinone also strengthens considerably when adsorbed to the $\mathrm{Cu}(111)$ surface (the $\mathrm{C} 1-\mathrm{C} 2$ bond is the bond between the two carbon atoms that are attached to the carbonyl oxygen atoms). In Figure S14, we show the how the length of the C1-C2 bond changes when the geometry of an adsorbed $o$-benzoquinone molecule is optimized over several sites over a $\mathrm{Cu}(111)$ surface. It can be seen that the C1-C2 bond is always less than $1.48 \AA$, which is significantly shorter than the $1.57 \AA$ C1-C2 distance of an isolated gas-phase molecule.

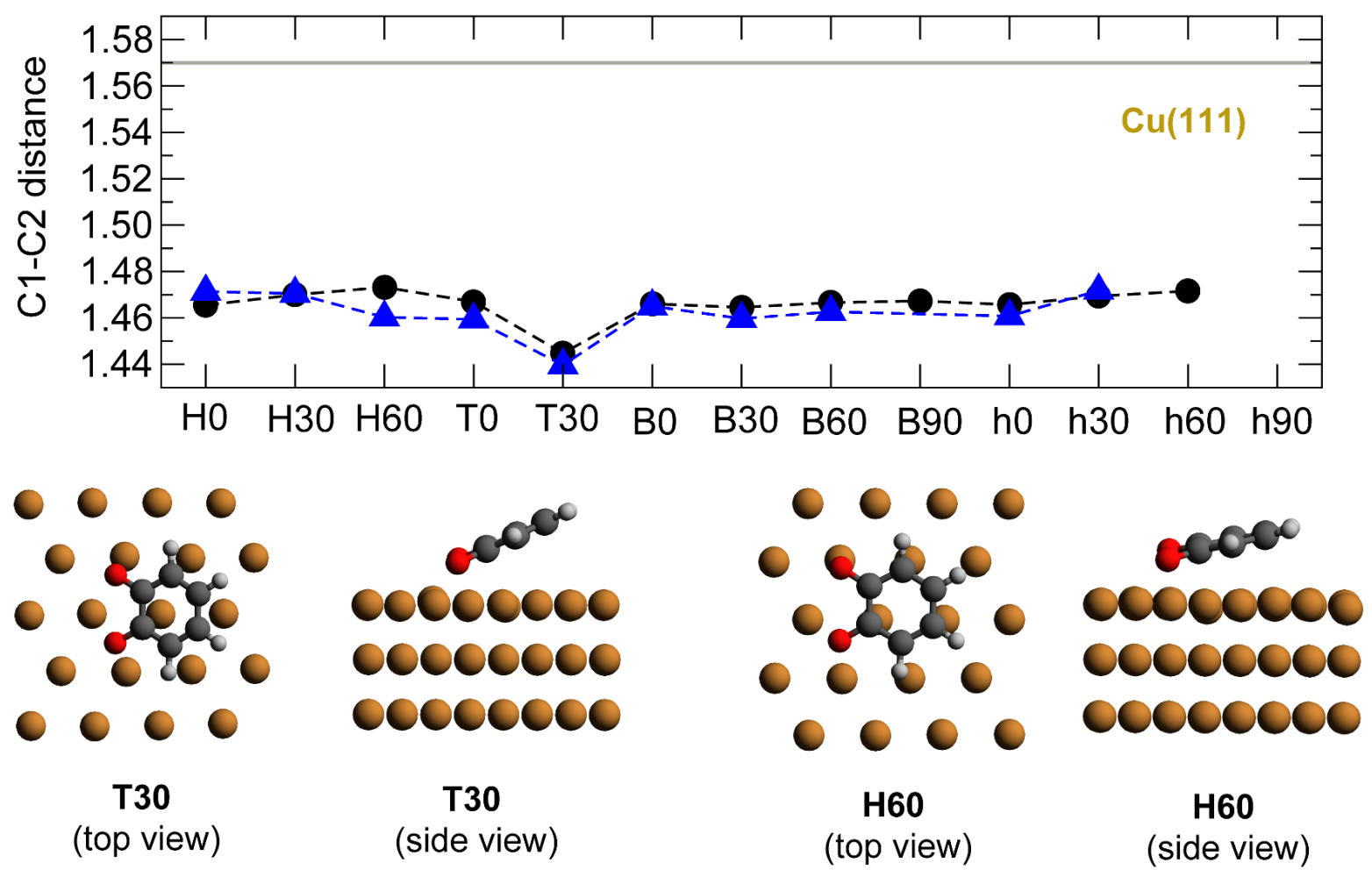

FIGURE S14: The bond length, in $\AA$, of the shrinking carbon-carbon bond in $o$-benzoquinone when adsorbed over several different sites on a $\mathrm{Cu}(111)$ substrate. $\mathrm{H}=\mathrm{H}_{\mathrm{fcc}}$, and $\mathrm{h}=\mathrm{H}_{\mathrm{hcp}}$. The black line was computed with the PBE-D3 functional, and the blue line was computed with the vdW-optB88 functional. 


\section{$\underline{\text { S12.SI References }}$}

SI Ref. 1: Miller, D.; Simpson, S.; Tymińska, N.; Zurek, E. J. Chem. Phys. 142, 101924 (2015).

SI Ref. 2: Kunkel, D.; Hooper, J.; Simpson, S.; Miller, D.; Routaboul, L.; Braunstein, P.; Doudin, B.; Beniwal, S.; Dowben, P.; Skomski, R.; Zurek, E.; Enders, A. J. Chem. Phys. 142, 101921 (2015). 


\section{S13.Coordinates}

Zwitterion- ADF Gas Phase

16

$\begin{array}{rrrr}\mathrm{O} & -2.392239 & 0.000000 & 5.045598 \\ \mathrm{O} & 2.392239 & 0.000000 & 5.045598 \\ \mathrm{~N} & -2.409704 & 0.000000 & 2.450128 \\ \mathrm{~N} & 2.409704 & 0.000000 & 2.450128 \\ \mathrm{C} & -1.192040 & 0.000000 & 2.987326 \\ \mathrm{C} & 0.000000 & 0.000000 & 5.213811 \\ \mathrm{C} & -1.238627 & 0.000000 & 4.538308 \\ \mathrm{C} & 1.192040 & 0.000000 & 2.987326 \\ \mathrm{C} & 0.000000 & 0.000000 & 2.258108 \\ \mathrm{C} & 1.238627 & 0.000000 & 4.538308 \\ \mathrm{H} & 0.000000 & 0.000000 & 1.162376 \\ \mathrm{H} & 0.000000 & 0.000000 & 6.303641 \\ \mathrm{H} & 3.171646 & 0.000000 & 3.139858 \\ \mathrm{H} & 2.580802 & 0.000000 & 1.452301 \\ \mathrm{H} & -2.580802 & 0.000000 & 1.452301 \\ \mathrm{H} & -3.171646 & 0.000000 & 3.139858\end{array}$

Zwitterion on Ag (160 Atom Cluster)-ADF 182

$\begin{array}{lccc}\text { Ag } & -1.668100 & -1.293210 & 2.889240 \\ \text { Ag } & -1.668100 & -1.293210 & 5.778480 \\ \text { Ag } & -1.668100 & -1.293210 & 8.667710 \\ \text { Ag } & -1.668100 & -1.293210 & 11.556950 \\ \text { Ag } & -1.668100 & -1.293210 & -11.556950 \\ \text { Ag } & -1.668100 & -1.293210 & -8.667710 \\ \text { Ag } & -1.668100 & -1.293210 & -5.778480 \\ \text { Ag } & -1.668100 & -1.293210 & -2.889240 \\ \text { Ag } & -1.668100 & -1.393220 & 0.000000 \\ \text { Ag } & -4.170260 & -1.293210 & 4.333860 \\ \text { Ag } & -4.170260 & -1.293210 & 7.223100 \\ \text { Ag } & -4.170250 & -1.293210 & 10.112330 \\ \text { Ag } & -4.170250 & -1.293210 & -10.112330 \\ \text { Ag } & -4.170260 & -1.293210 & -7.223100 \\ \text { Ag } & -4.170260 & -1.293210 & -4.333860 \\ \text { Ag } & -4.170260 & -1.293220 & -1.444620 \\ \mathrm{Ag} & -4.170260 & -1.293220 & 1.444620 \\ \mathrm{Ag} & -6.672410 & -1.293210 & 5.778480 \\ \mathrm{Ag} & -6.672410 & -1.293210 & 8.667700 \\ \mathrm{Ag} & -6.672410 & -1.293210 & -8.667700 \\ \mathrm{Ag} & -6.672410 & -1.293210 & -5.778480 \\ \mathrm{Ag} & -6.672410 & -1.293220 & -2.889240 \\ \mathrm{Ag} & -6.672410 & -1.293220 & 0.000000\end{array}$




\begin{tabular}{|c|c|c|c|}
\hline $\mathrm{Ag}$ & -6.672410 & -1.293220 & 2.889240 \\
\hline $\mathrm{Ag}$ & -9.174560 & -1.293210 & 7.223090 \\
\hline $\mathrm{Ag}$ & -9.174560 & -1.293210 & -7.223090 \\
\hline $\mathrm{Ag}$ & -9.174560 & -1.293230 & -4.333850 \\
\hline $\mathrm{Ag}$ & -9.174560 & -1.293230 & -1.444620 \\
\hline $\mathrm{Ag}$ & -9.174560 & -1.293230 & 1.444620 \\
\hline Ag & -9.174560 & -1.293230 & 4.333850 \\
\hline Ag & -11.676720 & -1.293230 & -5.778480 \\
\hline Ag & -11.676710 & -1.293230 & -2.889240 \\
\hline $\mathrm{Ag}$ & -11.676710 & -1.293230 & 0.000000 \\
\hline $\mathrm{Ag}$ & -11.676710 & -1.293230 & 2.889240 \\
\hline $\mathrm{Ag}$ & -11.676720 & -1.293230 & 5.778480 \\
\hline $\mathrm{Ag}$ & 10.842660 & -1.293210 & -4.333850 \\
\hline $\mathrm{Ag}$ & 10.842660 & -1.293210 & -1.444620 \\
\hline $\mathrm{Ag}$ & 10.842660 & -1.293210 & 1.444620 \\
\hline Ag & 10.842660 & -1.293210 & 4.333850 \\
\hline Ag & 10.842660 & -1.293230 & 7.223100 \\
\hline $\mathrm{Ag}$ & 10.842660 & -1.293230 & -7.223100 \\
\hline $\mathrm{Ag}$ & 8.340510 & -1.293210 & -2.889240 \\
\hline $\mathrm{Ag}$ & 8.340510 & -1.293210 & 0.000000 \\
\hline $\mathrm{Ag}$ & 8.340510 & -1.293210 & 2.889240 \\
\hline $\mathrm{Ag}$ & 8.340510 & -1.293230 & 5.778480 \\
\hline $\mathrm{Ag}$ & 8.340510 & -1.293230 & 8.667710 \\
\hline $\mathrm{Ag}$ & 8.340510 & -1.293230 & -8.667710 \\
\hline $\mathrm{Ag}$ & 8.340510 & -1.293230 & -5.778480 \\
\hline $\mathrm{Ag}$ & 5.838360 & -1.293210 & -1.444620 \\
\hline $\mathrm{Ag}$ & 5.838360 & -1.293210 & 1.444620 \\
\hline $\mathrm{Ag}$ & 5.838360 & -1.293220 & 4.333860 \\
\hline Ag & 5.838360 & -1.293230 & 7.223100 \\
\hline $\mathrm{Ag}$ & 5.838350 & -1.293230 & 10.112330 \\
\hline $\mathrm{Ag}$ & 5.838350 & -1.293230 & -10.112330 \\
\hline $\mathrm{Ag}$ & 5.838360 & -1.293230 & -7.223100 \\
\hline $\mathrm{Ag}$ & 5.838360 & -1.293220 & -4.333860 \\
\hline Ag & 3.336200 & -1.293210 & 0.000000 \\
\hline $\mathrm{Ag}$ & 3.336210 & -1.293220 & 2.889240 \\
\hline $\mathrm{Ag}$ & 3.336210 & -1.293220 & 5.778480 \\
\hline $\mathrm{Ag}$ & 3.336210 & -1.293230 & 8.667710 \\
\hline $\mathrm{Ag}$ & 3.336200 & -1.293230 & 11.556940 \\
\hline $\mathrm{Ag}$ & 3.336200 & -1.293230 & -11.556940 \\
\hline $\mathrm{Ag}$ & 3.336210 & -1.293230 & -8.667710 \\
\hline $\mathrm{Ag}$ & 3.336210 & -1.293220 & -5.778480 \\
\hline Ag & 3.336210 & -1.293220 & -2.889240 \\
\hline Ag & 0.834050 & -1.393220 & 1.444620 \\
\hline Ag & 0.834050 & -1.293220 & 4.333860 \\
\hline $\mathrm{Ag}$ & 0.834050 & -1.293220 & 7.223090 \\
\hline $\mathrm{Ag}$ & 0.834050 & -1.293230 & 10.112330 \\
\hline $\mathrm{Ag}$ & 0.834050 & -1.293230 & 13.001570 \\
\hline
\end{tabular}




\begin{tabular}{|c|c|c|c|}
\hline $\mathrm{Ag}$ & 0.834050 & -1.293230 & -13.001570 \\
\hline $\mathrm{Ag}$ & 0.834050 & -1.293230 & -10.112330 \\
\hline $\mathrm{Ag}$ & 0.834050 & -1.293220 & -7.223090 \\
\hline $\mathrm{Ag}$ & 0.834050 & -1.293220 & -4.333860 \\
\hline $\mathrm{Ag}$ & 0.834050 & -1.393220 & -1.444620 \\
\hline $\mathrm{Ag}$ & -2.502150 & 1.065840 & 4.333860 \\
\hline Ag & -2.502160 & 1.065840 & 7.223100 \\
\hline Ag & -2.502160 & 1.065840 & 10.112330 \\
\hline Ag & -2.502150 & 1.065840 & 13.001570 \\
\hline $\mathrm{Ag}$ & -2.502150 & 1.065840 & -13.001570 \\
\hline $\mathrm{Ag}$ & -2.502160 & 1.065840 & -10.112330 \\
\hline $\mathrm{Ag}$ & -2.502160 & 1.065840 & -7.223100 \\
\hline $\mathrm{Ag}$ & -2.502150 & 1.065840 & -4.333860 \\
\hline $\mathrm{Ag}$ & -2.502150 & 1.065830 & -1.444610 \\
\hline $\mathrm{Ag}$ & -2.502150 & 1.065830 & 1.444610 \\
\hline Ag & -5.004310 & 1.065840 & 5.778480 \\
\hline Ag & -5.004300 & 1.065840 & 8.667710 \\
\hline $\mathrm{Ag}$ & -5.004300 & 1.065840 & 11.556950 \\
\hline $\mathrm{Ag}$ & -5.004300 & 1.065840 & -11.556950 \\
\hline $\mathrm{Ag}$ & -5.004300 & 1.065840 & -8.667710 \\
\hline Ag & -5.004310 & 1.065840 & -5.778480 \\
\hline $\mathrm{Ag}$ & -5.004310 & 1.065830 & -2.889240 \\
\hline $\mathrm{Ag}$ & -5.004310 & 1.065830 & 0.000000 \\
\hline $\mathrm{Ag}$ & -5.004310 & 1.065830 & 2.889240 \\
\hline $\mathrm{Ag}$ & -7.506460 & 1.065840 & 7.223100 \\
\hline $\mathrm{Ag}$ & -7.506460 & 1.065840 & 10.112330 \\
\hline $\mathrm{Ag}$ & -7.506460 & 1.065840 & -10.112330 \\
\hline $\mathrm{Ag}$ & -7.506460 & 1.065840 & -7.223100 \\
\hline $\mathrm{Ag}$ & -7.506460 & 1.065820 & -4.333850 \\
\hline Ag & -7.506460 & 1.065830 & -1.444620 \\
\hline $\mathrm{Ag}$ & -7.506460 & 1.065830 & 1.444620 \\
\hline $\mathrm{Ag}$ & -7.506460 & 1.065820 & 4.333850 \\
\hline $\mathrm{Ag}$ & -10.008620 & 1.065840 & 8.667710 \\
\hline Ag & -10.008620 & 1.065840 & -8.667710 \\
\hline $\mathrm{Ag}$ & -10.008620 & 1.065820 & -5.778480 \\
\hline $\mathrm{Ag}$ & -10.008620 & 1.065820 & -2.889230 \\
\hline $\mathrm{Ag}$ & -10.008610 & 1.065820 & 0.000000 \\
\hline $\mathrm{Ag}$ & -10.008620 & 1.065820 & 2.889230 \\
\hline $\mathrm{Ag}$ & -10.008620 & 1.065820 & 5.778480 \\
\hline $\mathrm{Ag}$ & -12.510770 & 1.065810 & -7.223090 \\
\hline $\mathrm{Ag}$ & -12.510760 & 1.065820 & -4.333860 \\
\hline Ag & -12.510760 & 1.065820 & -1.444620 \\
\hline Ag & -12.510760 & 1.065820 & 1.444620 \\
\hline Ag & -12.510760 & 1.065820 & 4.333860 \\
\hline $\mathrm{Ag}$ & -12.510770 & 1.065810 & 7.223090 \\
\hline $\mathrm{Ag}$ & 12.510760 & 1.065840 & -4.333860 \\
\hline $\mathrm{Ag}$ & 12.510760 & 1.065840 & -1.444620 \\
\hline
\end{tabular}




\begin{tabular}{|c|c|c|c|}
\hline $\mathrm{Ag}$ & 12.510760 & 1.065840 & 1.444620 \\
\hline $\mathrm{Ag}$ & 12.510760 & 1.065840 & 4.333860 \\
\hline $\mathrm{Ag}$ & 12.510760 & 1.065810 & 7.223100 \\
\hline $\mathrm{Ag}$ & 12.510760 & 1.065810 & -7.223100 \\
\hline $\mathrm{Ag}$ & 10.008620 & 1.065840 & -2.889230 \\
\hline $\mathrm{Ag}$ & 10.008610 & 1.065840 & 0.000000 \\
\hline Ag & 10.008620 & 1.065840 & 2.889230 \\
\hline Ag & 10.008620 & 1.065820 & 5.778480 \\
\hline Ag & 10.008620 & 1.065820 & 8.667710 \\
\hline $\mathrm{Ag}$ & 10.008620 & 1.065820 & -8.667710 \\
\hline $\mathrm{Ag}$ & 10.008620 & 1.065820 & -5.778480 \\
\hline $\mathrm{Ag}$ & 7.506460 & 1.065840 & -1.444620 \\
\hline $\mathrm{Ag}$ & 7.506460 & 1.065840 & 1.444620 \\
\hline $\mathrm{Ag}$ & 7.506460 & 1.065820 & 4.333860 \\
\hline $\mathrm{Ag}$ & 7.506460 & 1.065820 & 7.223100 \\
\hline Ag & 7.506460 & 1.065820 & 10.112330 \\
\hline Ag & 7.506460 & 1.065820 & -10.112330 \\
\hline $\mathrm{Ag}$ & 7.506460 & 1.065820 & -7.223100 \\
\hline $\mathrm{Ag}$ & 7.506460 & 1.065820 & -4.333860 \\
\hline $\mathrm{Ag}$ & 5.004310 & 1.065840 & 0.000000 \\
\hline Ag & 5.004310 & 1.065830 & 2.889240 \\
\hline $\mathrm{Ag}$ & 5.004310 & 1.065830 & 5.778480 \\
\hline $\mathrm{Ag}$ & 5.004300 & 1.065820 & 8.667710 \\
\hline $\mathrm{Ag}$ & 5.004300 & 1.065820 & 11.556950 \\
\hline Ag & 5.004300 & 1.065820 & -11.556950 \\
\hline $\mathrm{Ag}$ & 5.004300 & 1.065820 & -8.667710 \\
\hline $\mathrm{Ag}$ & 5.004310 & 1.065830 & -5.778480 \\
\hline $\mathrm{Ag}$ & 5.004310 & 1.065830 & -2.889240 \\
\hline Ag & 2.502150 & 1.065830 & 1.444620 \\
\hline Ag & 2.502150 & 1.065830 & 4.333860 \\
\hline $\mathrm{Ag}$ & 2.502160 & 1.065830 & 7.223100 \\
\hline $\mathrm{Ag}$ & 2.502160 & 1.065820 & 10.112330 \\
\hline $\mathrm{Ag}$ & 2.502150 & 1.065820 & 13.001570 \\
\hline Ag & 2.502150 & 1.065820 & -13.001570 \\
\hline $\mathrm{Ag}$ & 2.502160 & 1.065820 & -10.112330 \\
\hline $\mathrm{Ag}$ & 2.502160 & 1.065830 & -7.223100 \\
\hline $\mathrm{Ag}$ & 2.502150 & 1.065830 & -4.333860 \\
\hline $\mathrm{Ag}$ & 2.502150 & 1.065830 & -1.444620 \\
\hline $\mathrm{Ag}$ & 0.000000 & 1.065830 & 2.889240 \\
\hline $\mathrm{Ag}$ & 0.000000 & 1.065830 & 5.778480 \\
\hline $\mathrm{Ag}$ & 0.000000 & 1.065820 & 8.667710 \\
\hline Ag & 0.000000 & 1.065820 & 11.556960 \\
\hline Ag & 0.000000 & 1.065810 & 14.446190 \\
\hline Ag & 0.000000 & 1.065810 & -14.446190 \\
\hline Ag & 0.000000 & 1.065820 & -11.556960 \\
\hline $\mathrm{Ag}$ & 0.000000 & 1.065820 & -8.667710 \\
\hline $\mathrm{Ag}$ & 0.000000 & 1.065830 & -5.778480 \\
\hline
\end{tabular}




$\begin{array}{lccc}\mathrm{Ag} & 0.000000 & 1.065830 & -2.889240 \\ \mathrm{Ag} & 0.000000 & 1.165840 & 0.000000 \\ \mathrm{C} & 1.007119 & 4.188488 & 1.211499 \\ \mathrm{C} & -1.151804 & 3.903061 & 0.000000 \\ \mathrm{C} & -0.480775 & 3.963328 & 1.249840 \\ \mathrm{O} & -1.019041 & 3.725118 & 2.387040 \\ \mathrm{~N} & 1.608763 & 4.169545 & 2.420703 \\ \mathrm{C} & 1.007119 & 4.188488 & -1.211499 \\ \mathrm{C} & 1.706699 & 4.282066 & 0.000000 \\ \mathrm{C} & -0.480775 & 3.963328 & -1.249840 \\ \mathrm{O} & -1.019041 & 3.725118 & -2.387040 \\ \mathrm{~N} & 1.608763 & 4.169545 & -2.420703 \\ \mathrm{H} & 2.797144 & 4.371975 & 0.000000 \\ \mathrm{H} & -2.232456 & 3.759213 & 0.000000 \\ \mathrm{H} & 1.005456 & 3.946034 & -3.213987 \\ \mathrm{H} & 2.613402 & 4.071261 & -2.521383 \\ \mathrm{H} & 2.613402 & 4.071261 & 2.521383 \\ \mathrm{H} & 1.005456 & 3.946034 & 3.213987\end{array}$

Zwitterion on $\mathrm{Cu}$ (160 atom cluster)-ADF 182

$\begin{array}{lrrl}\mathrm{Cu} & 8.105765 & -6.381640 & -1.142575 \\ \mathrm{Cu} & 8.105765 & 6.381640 & -1.142575 \\ \mathrm{Cu} & 1.473780 & -10.210618 & -1.142575 \\ \mathrm{Cu} & 1.473780 & 10.210618 & -1.142575 \\ \mathrm{Cu} & -9.579545 & 3.828978 & -1.142575 \\ \mathrm{Cu} & -9.579545 & -3.828978 & -1.142575 \\ \mathrm{Cu} & 5.895106 & -7.657964 & -1.142573 \\ \mathrm{Cu} & 5.895106 & 7.657964 & -1.142573 \\ \mathrm{Cu} & 3.684439 & -8.934294 & -1.142573 \\ \mathrm{Cu} & 3.684439 & 8.934294 & -1.142573 \\ \mathrm{Cu} & -9.579545 & 1.276329 & -1.142573 \\ \mathrm{Cu} & -9.579545 & -1.276329 & -1.142573 \\ \mathrm{Cu} & 5.895105 & -5.105304 & -1.142571 \\ \mathrm{Cu} & 5.895105 & 5.105304 & -1.142571 \\ \mathrm{Cu} & 1.473770 & -7.657963 & -1.142571 \\ \mathrm{Cu} & 1.473770 & 7.657963 & -1.142571 \\ \mathrm{Cu} & -7.368876 & 2.552659 & -1.142571 \\ \mathrm{Cu} & -7.368876 & -2.552659 & -1.142571 \\ \mathrm{Cu} & 10.316433 & 5.105310 & -1.142569 \\ \mathrm{Cu} & 10.316433 & -5.105310 & -1.142569 \\ \mathrm{Cu} & 3.684438 & -6.381633 & -1.142569 \\ \mathrm{Cu} & 3.684438 & 6.381633 & -1.142569 \\ \mathrm{Cu} & -0.736888 & -11.486948 & -1.142569 \\ \mathrm{Cu} & -0.736888 & 11.486948 & -1.142569 \\ \mathrm{Cu} & -7.368876 & 0.000000 & -1.142569\end{array}$




\begin{tabular}{|c|c|c|c|}
\hline $\mathrm{Cu}$ & -9.579545 & -6.381637 & -1.142569 \\
\hline $\mathrm{Cu}$ & -9.579545 & 6.381637 & -1.142569 \\
\hline $\mathrm{Cu}$ & 3.684442 & -3.828982 & -1.142567 \\
\hline $\mathrm{Cu}$ & 3.684442 & 3.828982 & -1.142567 \\
\hline $\mathrm{Cu}$ & 1.473774 & -5.105311 & -1.142567 \\
\hline $\mathrm{Cu}$ & 1.473774 & 5.105311 & -1.142567 \\
\hline $\mathrm{Cu}$ & -5.158216 & 1.276329 & -1.142567 \\
\hline $\mathrm{Cu}$ & -5.158216 & -1.276329 & -1.142567 \\
\hline $\mathrm{Cu}$ & 8.105764 & 3.828979 & -1.142566 \\
\hline $\mathrm{Cu}$ & 8.105764 & -3.828979 & -1.142566 \\
\hline $\mathrm{Cu}$ & -0.736889 & -8.934287 & -1.142566 \\
\hline $\mathrm{Cu}$ & -0.736889 & 8.934287 & -1.142566 \\
\hline $\mathrm{Cu}$ & -7.368876 & -5.105308 & -1.142566 \\
\hline $\mathrm{Cu}$ & -7.368876 & 5.105308 & -1.142566 \\
\hline $\mathrm{Cu}$ & 10.316432 & 2.552650 & -1.142565 \\
\hline $\mathrm{Cu}$ & 10.316432 & -2.552650 & -1.142565 \\
\hline $\mathrm{Cu}$ & -2.947556 & -10.210617 & -1.142565 \\
\hline $\mathrm{Cu}$ & -2.947556 & 10.210617 & -1.142565 \\
\hline $\mathrm{Cu}$ & -7.368876 & -7.657967 & -1.142565 \\
\hline $\mathrm{Cu}$ & -7.368876 & 7.657967 & -1.142565 \\
\hline $\mathrm{Cu}$ & 5.895101 & -2.552657 & -1.142564 \\
\hline $\mathrm{Cu}$ & 5.895101 & 2.552657 & -1.142564 \\
\hline $\mathrm{Cu}$ & -0.736884 & -6.381636 & -1.142564 \\
\hline $\mathrm{Cu}$ & -0.736884 & 6.381636 & -1.142564 \\
\hline $\mathrm{Cu}$ & -5.158216 & -3.828978 & -1.142564 \\
\hline $\mathrm{Cu}$ & -5.158216 & 3.828978 & -1.142564 \\
\hline $\mathrm{Cu}$ & 8.105768 & -1.276328 & -1.142563 \\
\hline $\mathrm{Cu}$ & 8.105768 & 1.276328 & -1.142563 \\
\hline $\mathrm{Cu}$ & 1.473774 & -2.552651 & -1.142563 \\
\hline $\mathrm{Cu}$ & 1.473774 & 2.552651 & -1.142563 \\
\hline $\mathrm{Cu}$ & -2.947547 & 0.000000 & -1.142563 \\
\hline $\mathrm{Cu}$ & -2.947552 & -7.657965 & -1.142563 \\
\hline $\mathrm{Cu}$ & -2.947552 & 7.657965 & -1.142563 \\
\hline $\mathrm{Cu}$ & -5.158216 & -6.381637 & -1.142563 \\
\hline $\mathrm{Cu}$ & -5.158216 & 6.381637 & -1.142563 \\
\hline $\mathrm{Cu}$ & 3.684441 & -1.276321 & -1.142562 \\
\hline $\mathrm{Cu}$ & 3.684441 & 1.276321 & -1.142562 \\
\hline $\mathrm{Cu}$ & -0.736894 & -3.828980 & -1.142562 \\
\hline $\mathrm{Cu}$ & -0.736894 & 3.828980 & -1.142562 \\
\hline $\mathrm{Cu}$ & -2.947547 & -2.552659 & -1.142562 \\
\hline $\mathrm{Cu}$ & -2.929936 & 2.540996 & -1.290662 \\
\hline $\mathrm{Cu}$ & 10.316427 & 0.000000 & -1.142561 \\
\hline $\mathrm{Cu}$ & -5.158214 & -8.934288 & -1.142561 \\
\hline $\mathrm{Cu}$ & -5.158214 & 8.934288 & -1.142561 \\
\hline $\mathrm{Cu}$ & 5.895100 & 0.000000 & -1.142560 \\
\hline $\mathrm{Cu}$ & 1.473778 & 0.000000 & -1.142560 \\
\hline $\mathrm{Cu}$ & -0.736889 & -1.276329 & -1.142560 \\
\hline
\end{tabular}




\begin{tabular}{|c|c|c|c|}
\hline $\mathrm{Cu}$ & -0.736889 & 1.276329 & -1.142560 \\
\hline $\mathrm{Cu}$ & -2.947550 & -5.105306 & -1.142560 \\
\hline $\mathrm{Cu}$ & -2.947550 & 5.105306 & -1.142560 \\
\hline $\mathrm{Cu}$ & 8.842651 & -7.657964 & 0.941652 \\
\hline $\mathrm{Cu}$ & 8.842651 & 7.657964 & 0.941652 \\
\hline $\mathrm{Cu}$ & 2.210666 & -11.486943 & 0.941652 \\
\hline $\mathrm{Cu}$ & 2.210666 & 11.486943 & 0.941652 \\
\hline $\mathrm{Cu}$ & -11.053317 & 3.828978 & 0.941652 \\
\hline $\mathrm{Cu}$ & -11.053317 & -3.828978 & 0.941652 \\
\hline $\mathrm{Cu}$ & 6.631992 & -8.934289 & 0.941654 \\
\hline $\mathrm{Cu}$ & 6.631992 & 8.934289 & 0.941654 \\
\hline $\mathrm{Cu}$ & 4.421325 & -10.210618 & 0.941654 \\
\hline $\mathrm{Cu}$ & 4.421325 & 10.210618 & 0.941654 \\
\hline $\mathrm{Cu}$ & -11.053317 & 1.276329 & 0.941654 \\
\hline $\mathrm{Cu}$ & -11.053317 & -1.276329 & 0.941654 \\
\hline $\mathrm{Cu}$ & 6.631996 & -6.381637 & 0.941656 \\
\hline $\mathrm{Cu}$ & 6.631996 & 6.381637 & 0.941656 \\
\hline $\mathrm{Cu}$ & 2.210661 & -8.934296 & 0.941656 \\
\hline $\mathrm{Cu}$ & 2.210661 & 8.934296 & 0.941656 \\
\hline $\mathrm{Cu}$ & -8.842658 & 2.552659 & 0.941656 \\
\hline $\mathrm{Cu}$ & -8.842658 & -2.552659 & 0.941656 \\
\hline $\mathrm{Cu}$ & 11.053319 & 6.381635 & 0.941658 \\
\hline $\mathrm{Cu}$ & 11.053319 & -6.381635 & 0.941658 \\
\hline $\mathrm{Cu}$ & 4.421329 & -7.657966 & 0.941658 \\
\hline $\mathrm{Cu}$ & 4.421329 & 7.657966 & 0.941658 \\
\hline $\mathrm{Cu}$ & -0.000002 & -12.763272 & 0.941658 \\
\hline $\mathrm{Cu}$ & -0.000002 & 12.763272 & 0.941658 \\
\hline $\mathrm{Cu}$ & -8.842658 & 0.000000 & 0.941658 \\
\hline $\mathrm{Cu}$ & -11.053317 & -6.381637 & 0.941658 \\
\hline $\mathrm{Cu}$ & -11.053317 & 6.381637 & 0.941658 \\
\hline $\mathrm{Cu}$ & 4.421328 & -5.105306 & 0.941660 \\
\hline $\mathrm{Cu}$ & 4.421328 & 5.105306 & 0.941660 \\
\hline $\mathrm{Cu}$ & 2.210661 & -6.381635 & 0.941660 \\
\hline $\mathrm{Cu}$ & 2.210661 & 6.381635 & 0.941660 \\
\hline $\mathrm{Cu}$ & -6.631989 & 1.276329 & 0.941660 \\
\hline $\mathrm{Cu}$ & -6.631989 & -1.276329 & 0.941660 \\
\hline $\mathrm{Cu}$ & 8.842655 & 5.105312 & 0.941661 \\
\hline $\mathrm{Cu}$ & 8.842655 & -5.105312 & 0.941661 \\
\hline $\mathrm{Cu}$ & 0.000003 & -10.210620 & 0.941661 \\
\hline $\mathrm{Cu}$ & 0.000003 & 10.210620 & 0.941661 \\
\hline $\mathrm{Cu}$ & -8.842658 & -5.105308 & 0.941661 \\
\hline $\mathrm{Cu}$ & -8.842658 & 5.105308 & 0.941661 \\
\hline $\mathrm{Cu}$ & 11.053323 & 3.828983 & 0.941662 \\
\hline $\mathrm{Cu}$ & 11.053323 & -3.828983 & 0.941662 \\
\hline $\mathrm{Cu}$ & -2.210665 & -11.486950 & 0.941662 \\
\hline $\mathrm{Cu}$ & -2.210665 & 11.486950 & 0.941662 \\
\hline $\mathrm{Cu}$ & -8.842658 & -7.657967 & 0.941662 \\
\hline
\end{tabular}




\begin{tabular}{|c|c|c|c|}
\hline $\mathrm{Cu}$ & -8.842658 & 7.657967 & 0.941662 \\
\hline $\mathrm{Cu}$ & 6.631987 & 3.828981 & 0.941663 \\
\hline $\mathrm{Cu}$ & 6.631987 & -3.828981 & 0.941663 \\
\hline $\mathrm{Cu}$ & 0.000002 & -7.657960 & 0.941663 \\
\hline $\mathrm{Cu}$ & 0.000002 & 7.657960 & 0.941663 \\
\hline $\mathrm{Cu}$ & -6.631989 & -3.828978 & 0.941663 \\
\hline $\mathrm{Cu}$ & -6.631989 & 3.828978 & 0.941663 \\
\hline $\mathrm{Cu}$ & 2.210665 & -3.828984 & 0.941664 \\
\hline $\mathrm{Cu}$ & 2.210665 & 3.828984 & 0.941664 \\
\hline $\mathrm{Cu}$ & -4.421330 & 0.000000 & 0.941664 \\
\hline $\mathrm{Cu}$ & 8.842655 & 2.552652 & 0.941665 \\
\hline $\mathrm{Cu}$ & 8.842655 & -2.552652 & 0.941665 \\
\hline $\mathrm{Cu}$ & 4.421332 & -2.552654 & 0.941665 \\
\hline $\mathrm{Cu}$ & 4.421332 & 2.552654 & 0.941665 \\
\hline $\mathrm{Cu}$ & -0.000003 & -5.105313 & 0.941665 \\
\hline $\mathrm{Cu}$ & -0.000003 & 5.105313 & 0.941665 \\
\hline $\mathrm{Cu}$ & -2.210666 & -8.934289 & 0.941665 \\
\hline $\mathrm{Cu}$ & -2.210666 & 8.934289 & 0.941665 \\
\hline $\mathrm{Cu}$ & -4.421330 & -2.552659 & 0.941665 \\
\hline $\mathrm{Cu}$ & -4.421330 & 2.552659 & 0.941665 \\
\hline $\mathrm{Cu}$ & -6.631989 & -6.381637 & 0.941665 \\
\hline $\mathrm{Cu}$ & -6.631989 & 6.381637 & 0.941665 \\
\hline $\mathrm{Cu}$ & 11.053313 & 1.276327 & 0.941666 \\
\hline $\mathrm{Cu}$ & 11.053313 & -1.276327 & 0.941666 \\
\hline $\mathrm{Cu}$ & -4.421325 & -10.210614 & 0.941666 \\
\hline $\mathrm{Cu}$ & -4.421325 & 10.210614 & 0.941666 \\
\hline $\mathrm{Cu}$ & -6.631989 & -8.934286 & 0.941666 \\
\hline $\mathrm{Cu}$ & -6.631989 & 8.934286 & 0.941666 \\
\hline $\mathrm{Cu}$ & 6.631991 & -1.276330 & 0.941667 \\
\hline $\mathrm{Cu}$ & 6.631991 & 1.276330 & 0.941667 \\
\hline $\mathrm{Cu}$ & -2.210662 & -6.381638 & 0.941667 \\
\hline $\mathrm{Cu}$ & -2.210662 & 6.381638 & 0.941667 \\
\hline $\mathrm{Cu}$ & -4.421330 & -5.105308 & 0.941667 \\
\hline $\mathrm{Cu}$ & -4.421330 & 5.105308 & 0.941667 \\
\hline $\mathrm{Cu}$ & 8.842659 & 0.000000 & 0.941668 \\
\hline $\mathrm{Cu}$ & 2.210664 & -1.276323 & 0.941668 \\
\hline $\mathrm{Cu}$ & 2.210664 & 1.276323 & 0.941668 \\
\hline $\mathrm{Cu}$ & -0.000004 & -2.552653 & 0.941668 \\
\hline $\mathrm{Cu}$ & -0.000004 & 2.552653 & 0.941668 \\
\hline $\mathrm{Cu}$ & -2.210660 & -1.276329 & 0.941668 \\
\hline $\mathrm{Cu}$ & -2.210660 & 1.276329 & 0.941668 \\
\hline $\mathrm{Cu}$ & -4.421329 & -7.657967 & 0.941668 \\
\hline $\mathrm{Cu}$ & -4.421329 & 7.657967 & 0.941668 \\
\hline $\mathrm{Cu}$ & 4.421323 & 0.000000 & 0.941669 \\
\hline $\mathrm{Cu}$ & -2.210661 & -3.828978 & 0.941669 \\
\hline $\mathrm{Cu}$ & -2.210661 & 3.828978 & 0.941669 \\
\hline $\mathrm{Cu}$ & 0.000000 & 0.000000 & 0.941670 \\
\hline
\end{tabular}




$\begin{array}{lrrr}\mathrm{C} & -0.580758 & -1.241294 & 3.508926 \\ \mathrm{C} & -2.724497 & -0.021946 & 3.463838 \\ \mathrm{C} & -2.038966 & -1.278087 & 3.440669 \\ \mathrm{O} & -2.632412 & -2.423270 & 3.213722 \\ \mathrm{~N} & 0.050146 & -2.473619 & 3.246232 \\ \mathrm{C} & -0.584706 & 1.207932 & 3.510435 \\ \mathrm{C} & 0.121089 & -0.015198 & 3.515162 \\ \mathrm{C} & -2.043802 & 1.237708 & 3.444941 \\ \mathrm{O} & -2.641902 & 2.380454 & 3.225027 \\ \mathrm{~N} & 0.039617 & 2.444860 & 3.253823 \\ \mathrm{H} & 1.215800 & -0.013300 & 3.483453 \\ \mathrm{H} & -3.816963 & -0.024152 & 3.466601 \\ \mathrm{H} & -0.555998 & 3.234408 & 3.514151 \\ \mathrm{H} & 1.010696 & 2.543851 & 3.541272 \\ \mathrm{H} & 1.023074 & -2.566725 & 3.529907 \\ \mathrm{H} & -0.538964 & -3.268026 & 3.506886\end{array}$

Zwitterion on Au (160 Atom cluster)-ADF 182

$\begin{array}{llll}\mathrm{Au} & -2.355589 & -1.665653 & 0.000000 \\ \mathrm{Au} & -2.355589 & -4.164133 & 1.442500 \\ \mathrm{Au} & -2.355589 & -6.662612 & 2.885000 \\ \mathrm{Au} & -2.355589 & -9.161092 & 4.327490 \\ \mathrm{Au} & -2.355589 & -11.659571 & 5.769990 \\ \mathrm{Au} & -2.355589 & 10.826745 & -7.212490 \\ \mathrm{Au} & -2.355589 & 8.328265 & -5.769990 \\ \mathrm{Au} & -2.355589 & 5.829786 & -4.327490 \\ \mathrm{Au} & -2.355589 & 3.331306 & -2.885000 \\ \mathrm{Au} & -2.355589 & 0.832827 & -1.442500 \\ \mathrm{Au} & -2.355589 & -1.665653 & 2.885000 \\ \mathrm{Au} & -2.355589 & -4.164133 & 4.327490 \\ \mathrm{Au} & -2.355589 & -6.662612 & 5.769990 \\ \mathrm{Au} & -2.355589 & -9.161092 & 7.212490 \\ \mathrm{Au} & -2.355589 & 10.826745 & -4.327490 \\ \mathrm{Au} & -2.355589 & 8.328265 & -2.885000 \\ \mathrm{Au} & -2.355589 & 5.829786 & -1.442500 \\ \mathrm{Au} & -2.355589 & 3.331306 & 0.000000 \\ \mathrm{Au} & -2.355589 & 0.832827 & 1.442500 \\ \mathrm{Au} & -2.355589 & -1.665653 & 5.769990 \\ \mathrm{Au} & -2.355589 & -4.164133 & 7.212490 \\ \mathrm{Au} & -2.355589 & -6.662612 & 8.654990 \\ \mathrm{Au} & -2.355589 & 10.826745 & -1.442500 \\ \mathrm{Au} & -2.355589 & 8.328265 & 0.000000 \\ \mathrm{Au} & -2.355589 & 5.829786 & 1.442500 \\ \mathrm{Au} & -2.355589 & 3.331306 & 2.885000 \\ \mathrm{Au} & -2.355589 & 0.832827 & 4.327490\end{array}$




\begin{tabular}{|c|c|c|c|}
\hline $\mathrm{Au}$ & -2.355589 & -1.665653 & 8.654990 \\
\hline $\mathrm{Au}$ & -2.355589 & -4.164133 & 10.097500 \\
\hline $\mathrm{Au}$ & -2.355589 & 10.826745 & 1.442500 \\
\hline $\mathrm{Au}$ & -2.355589 & 8.328265 & 2.885000 \\
\hline $\mathrm{Au}$ & -2.355589 & 5.829786 & 4.327490 \\
\hline $\mathrm{Au}$ & -2.355589 & 3.331306 & 5.769990 \\
\hline $\mathrm{Au}$ & -2.355589 & 0.832827 & 7.212490 \\
\hline . & -2.355589 & -1.665653 & 11.540000 \\
\hline $\mathrm{Au}$ & -2.355589 & 10.826745 & 4.327490 \\
\hline $\mathrm{Au}$ & -2.355589 & 8.328265 & 5.769990 \\
\hline $\mathrm{Au}$ & -2.35 & 5.829786 & 7.212490 \\
\hline $\mathrm{u}$ & -2.355589 & 3.331306 & 8.654990 \\
\hline u & -2.3 & 0.832827 & 10.097500 \\
\hline $\mathrm{Au}$ & -2.35 & 10.826745 & 7.212490 \\
\hline $\mathrm{Au}$ & -2.355589 & 8.328265 & 8.654990 \\
\hline $\mathrm{Au}$ & -2.35 & 5.829786 & 10.097500 \\
\hline $\mathrm{Au}$ & -2.355589 & 3.331306 & 11.540000 \\
\hline $\mathrm{Au}$ & -2.3 & 0.832827 & 12.982500 \\
\hline $\mathrm{Au}$ & -2.355589 & -1.665653 & -11.540000 \\
\hline $\mathrm{u}$ & -2.35 & -4.164133 & -10.097500 \\
\hline $\mathrm{Au}$ & -2.355589 & -6.662612 & -8.654990 \\
\hline $\mathrm{u}$ & -2.355589 & -9.161092 & -7.212490 \\
\hline $\mathrm{Au}$ & -2.355589 & -11.659571 & -5.769990 \\
\hline $\mathrm{Al}$ & -2.355589 & 0.832827 & -12.982500 \\
\hline $\mathrm{Au}$ & -2.355589 & -1.665653 & -8.654990 \\
\hline $\mathrm{Au}$ & -2.355589 & -4.164133 & -7.212490 \\
\hline $\mathrm{Au}$ & -2.355589 & -6.662612 & -5.769990 \\
\hline $\mathrm{Au}$ & -2.355589 & -9.161092 & -4.327490 \\
\hline $\mathrm{Au}$ & -2.355589 & -11.659571 & -2.885000 \\
\hline $\mathrm{Au}$ & -2.355589 & 3.331306 & -11.540000 \\
\hline $\mathrm{Au}$ & -2.355589 & 0.832827 & -10.097500 \\
\hline $\mathrm{Au}$ & -2.355589 & -1.665653 & -5.769990 \\
\hline $\mathrm{Au}$ & -2.355589 & -4.164133 & -4.327490 \\
\hline $\mathrm{Au}$ & -2.355589 & -6.662612 & -2.885000 \\
\hline $\mathrm{Au}$ & -2.355589 & -9.161092 & -1.442500 \\
\hline $\mathrm{Au}$ & -2.355589 & -11.659571 & 0.000000 \\
\hline $\mathrm{Au}$ & -2.355589 & 5.829786 & -10.097500 \\
\hline $\mathrm{Au}$ & -2.355589 & 3.331306 & -8.654990 \\
\hline $\mathrm{Au}$ & -2.355589 & 0.832827 & -7.212490 \\
\hline $\mathrm{Au}$ & -2.355589 & -1.665653 & -2.885000 \\
\hline $\mathrm{Au}$ & -2.355589 & -4.164133 & -1.442500 \\
\hline $\mathrm{Au}$ & -2.355589 & -6.662612 & 0.000000 \\
\hline $\mathrm{Au}$ & -2.355589 & -9.161092 & 1.442500 \\
\hline $\mathrm{Au}$ & -2.355589 & -11.659571 & 2.885000 \\
\hline $\mathrm{Au}$ & -2.355589 & 8.328265 & -8.654990 \\
\hline Al & -2.355589 & 5.829786 & -7.212490 \\
\hline Al & -2.355589 & 3.331306 & -5.769990 \\
\hline
\end{tabular}




\begin{tabular}{|c|c|c|c|}
\hline $\mathrm{Au}$ & -2.355589 & 0.832827 & -4.327490 \\
\hline $\mathrm{Au}$ & 0.000000 & -2.498480 & 1.442500 \\
\hline $\mathrm{Au}$ & 0.000000 & -4.996959 & 2.885000 \\
\hline $\mathrm{Au}$ & 0.000000 & -7.495439 & 4.327490 \\
\hline $\mathrm{Au}$ & 0.000000 & -9.993918 & 5.769990 \\
\hline $\mathrm{Au}$ & 0.000000 & -12.492398 & 7.212490 \\
\hline $\mathrm{Au}$ & 0.000000 & 12.492398 & -7.212490 \\
\hline $\mathrm{Au}$ & 0.000000 & 9.993918 & -5.769990 \\
\hline $\mathrm{Au}$ & 0.000000 & 7.495439 & -4.327490 \\
\hline $\mathrm{Au}$ & 0.000000 & 4.996959 & -2.885000 \\
\hline $\mathrm{Au}$ & 0.000000 & 2.498480 & -1.442500 \\
\hline $\mathrm{Au}$ & 0.000000 & 0.000000 & 0.000000 \\
\hline $\mathrm{Au}$ & 0.000000 & -2.498480 & 4.327490 \\
\hline $\mathrm{Au}$ & 0.000000 & -4.996959 & 5.769990 \\
\hline $\mathrm{Au}$ & 0.000000 & -7.495439 & 7.212490 \\
\hline $\mathrm{Au}$ & 0.000000 & -9.993918 & 8.654990 \\
\hline $\mathrm{Au}$ & 0.000000 & 12.492398 & -4.327490 \\
\hline $\mathrm{Au}$ & 0.000000 & 9.993918 & -2.885000 \\
\hline $\mathrm{Au}$ & 0.000000 & 7.495439 & -1.442500 \\
\hline $\mathrm{Au}$ & 0.000000 & 4.996959 & 0.000000 \\
\hline $\mathrm{Au}$ & 0.000000 & 2.498480 & 1.442500 \\
\hline $\mathrm{Au}$ & 0.000000 & 0.000000 & 2.885000 \\
\hline $\mathrm{Au}$ & 0.000000 & -2.498480 & 7.212490 \\
\hline $\mathrm{Au}$ & 0.000000 & -4.996959 & 8.654990 \\
\hline $\mathrm{Au}$ & 0.000000 & -7.495439 & 10.097500 \\
\hline $\mathrm{Au}$ & 0.000000 & 12.492398 & -1.442500 \\
\hline $\mathrm{Au}$ & 0.000000 & 9.993918 & 0.000000 \\
\hline $\mathrm{Au}$ & 0.000000 & 7.495439 & 1.442500 \\
\hline $\mathrm{Au}$ & 0.000000 & 4.996959 & 2.885000 \\
\hline $\mathrm{Au}$ & 0.000000 & 2.498480 & 4.327490 \\
\hline $\mathrm{Au}$ & 0.000000 & 0.000000 & 5.769990 \\
\hline $\mathrm{Au}$ & 0.000000 & -2.498480 & 10.097500 \\
\hline $\mathrm{Au}$ & 0.000000 & -4.996959 & 11.540000 \\
\hline $\mathrm{Au}$ & 0.000000 & 12.492398 & 1.442500 \\
\hline $\mathrm{Au}$ & 0.000000 & 9.993918 & 2.885000 \\
\hline $\mathrm{Au}$ & 0.000000 & 7.495439 & 4.327490 \\
\hline $\mathrm{Au}$ & 0.000000 & 4.996959 & 5.769990 \\
\hline $\mathrm{Au}$ & 0.000000 & 2.498480 & 7.212490 \\
\hline $\mathrm{Au}$ & 0.000000 & 0.000000 & 8.654990 \\
\hline $\mathrm{Au}$ & 0.000000 & -2.498480 & 12.982500 \\
\hline $\mathrm{Au}$ & 0.000000 & 12.492398 & 4.327490 \\
\hline $\mathrm{Au}$ & 0.000000 & 9.993918 & 5.769990 \\
\hline $\mathrm{Au}$ & 0.000000 & 7.495439 & 7.212490 \\
\hline $\mathrm{Au}$ & 0.000000 & 4.996959 & 8.654990 \\
\hline $\mathrm{Au}$ & 0.000000 & 2.498480 & 10.097500 \\
\hline $\mathrm{Au}$ & 0.000000 & 0.000000 & 11.540000 \\
\hline $\mathrm{Au}$ & 0.000000 & 12.492398 & 7.212490 \\
\hline
\end{tabular}




\begin{tabular}{|c|c|c|c|}
\hline $\mathrm{Au}$ & 0.000000 & 9.993918 & 8.654990 \\
\hline $\mathrm{Au}$ & 0.000000 & 7.495439 & 10.097500 \\
\hline $\mathrm{Au}$ & 0.000000 & 4.996959 & 11.540000 \\
\hline $\mathrm{Au}$ & 0.000000 & 2.498480 & 12.982500 \\
\hline $\mathrm{Au}$ & 0.000000 & 0.000000 & 14.425000 \\
\hline $\mathrm{Au}$ & 0.000000 & -2.498480 & -12.982500 \\
\hline $\mathrm{Au}$ & 0.000000 & -4.996959 & -11.540000 \\
\hline $\mathrm{Au}$ & 0.000000 & -7.495439 & -10.097500 \\
\hline $\mathrm{Au}$ & 0.000000 & -9.993918 & -8.654990 \\
\hline $\mathrm{Au}$ & 0.000000 & -12.492398 & -7.212490 \\
\hline $\mathrm{Au}$ & 0.000000 & 0.000000 & -14.425000 \\
\hline $\mathrm{Au}$ & 0.000000 & -2.498480 & -10.097500 \\
\hline $\mathrm{Au}$ & 0.000000 & -4.996959 & -8.654990 \\
\hline $\mathrm{Au}$ & 0.000000 & -7.495439 & -7.212490 \\
\hline $\mathrm{Au}$ & 0.000000 & -9.993918 & -5.769990 \\
\hline $\mathrm{Au}$ & 0.000000 & -12.492398 & - -4.327490 \\
\hline $\mathrm{Au}$ & 0.000000 & 2.498480 & -12.982500 \\
\hline $\mathrm{Au}$ & 0.000000 & 0.000000 & -11.540000 \\
\hline $\mathrm{Au}$ & 0.000000 & -2.498480 & -7.212490 \\
\hline $\mathrm{Au}$ & 0.000000 & -4.996959 & -5.769990 \\
\hline $\mathrm{Au}$ & 0.000000 & -7.495439 & -4.327490 \\
\hline $\mathrm{Au}$ & 0.000000 & -9.993918 & -2.885000 \\
\hline $\mathrm{Au}$ & 0.000000 & -12.492398 & -1.442500 \\
\hline $\mathrm{Au}$ & 0.000000 & 4.996959 & -11.540000 \\
\hline $\mathrm{Au}$ & 0.000000 & 2.498480 & -10.097500 \\
\hline $\mathrm{Au}$ & 0.000000 & 0.000000 & -8.654990 \\
\hline $\mathrm{Au}$ & 0.000000 & -2.498480 & -4.327490 \\
\hline $\mathrm{Au}$ & 0.000000 & -4.996959 & -2.885000 \\
\hline $\mathrm{Au}$ & 0.000000 & -7.495439 & -1.442500 \\
\hline $\mathrm{Au}$ & 0.000000 & -9.993918 & 0.000000 \\
\hline $\mathrm{Au}$ & 0.000000 & -12.492398 & 1.442500 \\
\hline $\mathrm{Au}$ & 0.000000 & 7.495439 & -10.097500 \\
\hline $\mathrm{Au}$ & 0.000000 & 4.996959 & -8.654990 \\
\hline $\mathrm{Au}$ & 0.000000 & 2.498480 & -7.212490 \\
\hline $\mathrm{Au}$ & 0.000000 & 0.000000 & -5.769990 \\
\hline $\mathrm{Au}$ & 0.000000 & -2.498480 & -1.442500 \\
\hline $\mathrm{Au}$ & 0.000000 & -4.996959 & 0.000000 \\
\hline $\mathrm{Au}$ & 0.000000 & -7.495439 & 1.442500 \\
\hline $\mathrm{Au}$ & 0.000000 & -9.993918 & 2.885000 \\
\hline $\mathrm{Au}$ & 0.000000 & -12.492398 & 4.327490 \\
\hline $\mathrm{Au}$ & 0.000000 & 9.993918 & -8.654990 \\
\hline $\mathrm{Au}$ & 0.000000 & 7.495439 & -7.212490 \\
\hline $\mathrm{Au}$ & 0.000000 & 4.996959 & -5.769990 \\
\hline $\mathrm{Au}$ & 0.000000 & 2.498480 & -4.327490 \\
\hline $\mathrm{Au}$ & 0.000000 & 0.000000 & -2.885000 \\
\hline $\mathrm{C}$ & 3.066463 & -0.652304 & 1.200954 \\
\hline $\mathrm{C}$ & 3.096359 & -2.869606 & 0.000000 \\
\hline
\end{tabular}




$\begin{array}{llrc}\mathrm{C} & 3.095827 & -2.191404 & 1.245332 \\ \mathrm{O} & 3.061384 & -2.715689 & 2.391930 \\ \mathrm{~N} & 3.051890 & -0.094899 & 2.407714 \\ \mathrm{C} & 3.066463 & -0.652304 & -1.200954 \\ \mathrm{C} & 2.976205 & 0.069144 & 0.000000 \\ \mathrm{C} & 3.095827 & -2.191404 & -1.245332 \\ \mathrm{O} & 3.061384 & -2.715689 & -2.391930 \\ \mathrm{~N} & 3.051890 & -0.094899 & -2.407714 \\ \mathrm{H} & 2.922452 & 1.160649 & 0.000000 \\ \mathrm{H} & 3.081056 & -3.959523 & 0.000000 \\ \mathrm{H} & 3.009413 & -0.759438 & -3.188508 \\ \mathrm{H} & 2.863332 & 0.891490 & -2.557115 \\ \mathrm{H} & 2.863332 & 0.891490 & 2.557115 \\ \mathrm{H} & 3.009413 & -0.759438 & 3.188508\end{array}$

Zwitterion on Ag (114 Atom Cluster, SP calc from VASP)-ADF 130

$\begin{array}{lccc}\text { O } & 23.1898102113 & 24.9102095190 & 9.7362101080 \\ \mathrm{O} & 19.0457980471 & 22.4745959868 & 9.7446037050 \\ \mathrm{H} & 20.5001492828 & 24.7451415080 & 9.8264102524 \\ \mathrm{C} & 21.0523518881 & 23.8049905719 & 9.8885911510 \\ \mathrm{C} & 22.4743616574 & 23.8557088519 & 9.9253497552 \\ \mathrm{C} & 20.3145777460 & 22.5892740019 & 9.9284436030 \\ \mathrm{H} & 24.9544490801 & 23.5442403284 & 10.0103474665 \\ \mathrm{H} & 19.3389206643 & 20.3104717162 & 10.0147727472 \\ \mathrm{~N} & 20.3507783370 & 20.1926218040 & 10.0560793707 \\ \mathrm{~N} & 24.5359413705 & 22.6173644753 & 10.0570340827 \\ \mathrm{C} & 23.1867628305 & 22.5468027565 & 10.1023995141 \\ \mathrm{C} & 21.0934140440 & 21.3223602012 & 10.1034903148 \\ \mathrm{H} & 25.1263488078 & 21.7986631880 & 10.1418303213 \\ \mathrm{H} & 20.7493706582 & 19.2679547350 & 10.1612851483 \\ \mathrm{C} & 22.4913073629 & 21.3300414845 & 10.1824348281 \\ \mathrm{H} & 23.0411775751 & 20.3890386210 & 10.2636229554 \\ \mathrm{Ag} & 29.0120000000 & 18.4259324280 & 2.4232800000 \\ \mathrm{Ag} & 29.0120000000 & 23.4508386080 & 2.4232800000 \\ \mathrm{Ag} & 29.0120000000 & 28.4757297120 & 2.4232800000 \\ \mathrm{Ag} & 24.6598663620 & 15.9134868760 & 2.4232800000 \\ \mathrm{Ag} & 27.5609793260 & 20.9383779800 & 2.4232800000 \\ \mathrm{Ag} & 27.5609793260 & 15.9134868760 & 2.4232800000 \\ \mathrm{Ag} & 18.8576694460 & 15.9134868760 & 2.4232800000 \\ \mathrm{Ag} & 21.7587679040 & 20.9383779800 & 2.4232800000 \\ \mathrm{Ag} & 21.7587679040 & 15.9134868760 & 2.4232800000 \\ \mathrm{Ag} & 24.6598663620 & 25.9632841600 & 2.4232800000 \\ \mathrm{Ag} & 24.6598663620 & 20.9383779800 & 2.4232800000 \\ \mathrm{Ag} & 26.1104228440 & 18.4259324280 & 2.4232800000 \\ \mathrm{Ag} & 23.2093243860 & 18.4259324280 & 2.4232800000\end{array}$




\begin{tabular}{|c|c|c|c|}
\hline & 7.5609793260 & 25.9632841600 & \\
\hline$g$ & 6.1104228440 & 23.4508386080 & 2.4232800000 \\
\hline & 5.9565564820 & 20.9383779800 & 4232800000 \\
\hline$g$ & 15.9565564820 & 15.9134868760 & 2.4232800000 \\
\hline & 8.8576694460 & 5.9632841600 & 2.423280000 \\
\hline & 18.8576694460 & 0.9383779800 & 00000 \\
\hline & 0.3082114220 & 8.4259324280 & 2.4232800000 \\
\hline & 17.4071129640 & 18.4259324280 & 00000 \\
\hline & 21.7587679040 & 32841600 & 2.42 \\
\hline & 23.2093243860 & 5080 & 2.4 \\
\hline & 0.3082114220 & 6080 & 2.4 \\
\hline & 26.1104228440 & 28.4757 & 00000 \\
\hline & 243860 & 120 & 00000 \\
\hline & 4.5060000000 & 4280 & 2.4 \\
\hline & 65564820 & 600 & 00000 \\
\hline & 17.4071129640 & 36080 & 0000 \\
\hline & 00000 & 5080 & \\
\hline & 20.3082114220 & 28.4 & 2.4 \\
\hline & 17.4071129640 & 28.4 & 0000 \\
\hline$A$ & 00000 & 20 & 2.4 \\
\hline & 26.1104 & 13.3 & 0000 \\
\hline & 243860 & 120 & 00000 \\
\hline & 20 & 120 & 0000 \\
\hline & 17.4071129640 & 7120 & 2.4 \\
\hline A & 23.2239 & 671 & 4.7 \\
\hline & 21. & 286 & \\
\hline g & 18.835 & 257 & 4.7 \\
\hline & 20.2810408215 & 0395 & 4.7 \\
\hline $\mathrm{Ag}$ & 21.7 & 2183 & 11485 \\
\hline & 26.12496783 & 9220 & 4.7 \\
\hline$g$ & 20.3069168806 & 4333 & 41456 \\
\hline & 24.66728470 & 6277 & 0622 \\
\hline $\mathrm{Ag}$ & 24.6602857497 & 4483 & 154042 \\
\hline 18 & 17.4080301139 & 26.7 & 3318 \\
\hline g & 21.7592 & 29.2 & 4.7 \\
\hline Ag & 21.7592463760 & 47405 & 79650 \\
\hline$\Delta$ & 18.8 & 7722 & 4.7 \\
\hline $\mathrm{Ag}$ & 18.85506416 & 7722 & 459731 \\
\hline 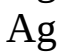 & 24.6613202944 & 29.2815 & 4.7901869554 \\
\hline $\mathrm{Ag}$ & 24.6613202944 & 19861 & 4.7901869554 \\
\hline$A$ & 15.9552526605 & 29.2555049889 & 4.7902847508 \\
\hline Ag & 15.9552526605 & 14.1795049889 & 4.7902847508 \\
\hline A o & 20.3069023294 & 16.7158317523 & 4.7908312236 \\
\hline $\mathrm{Ag}$ & 23.2116114002 & 16.7181201150 & 4.7916257015 \\
\hline $\mathrm{Ag}$ & 17.4015769985 & 16.7015569468 & 4.7929403186 \\
\hline & & 29.2 & 4.79 \\
\hline Ag & 27.5627050136 & 14.1891545060 & 4.793410969 \\
\hline
\end{tabular}




\begin{tabular}{|c|c|c|c|}
\hline Ag & 6.1158742465 & 26.7617594060 & $4.734100 / 121$ \\
\hline g & 5.9477275352 & 19.2064512319 & 47948984387 \\
\hline & 9.0114028701 & 26.7417945126 & 7952211984 \\
\hline$g$ & 4.5054028701 & 26.7417945126 & 47952211984 \\
\hline & 9.0099937425 & 21.7181877596 & 4.7955965175 \\
\hline & 14.5039937425 & 1.7181877596 & 4795596517 \\
\hline & 15.9550940672 & 24.2343864541 & 4.79 \\
\hline & 27.5616022756 & 24.2351476174 & 4.796 \\
\hline & .01007 & 16.69724 & 4.7 \\
\hline & 14.5040769356 & 16.6972477517 & 4.796093992 \\
\hline & 37 & 56 & \\
\hline & 7.3974232674 & 21.7201631667 & 109108 \\
\hline & 75 & 4.2261707167 & 4.7 \\
\hline & 672 & 26.7722602979 & 4.7974252 \\
\hline & 80 & 7075987 & 4.7 \\
\hline g & 21 & 22.5 & \\
\hline & 36 & 22.5711725557 & 90587 \\
\hline g & 73 & 20.01298 & 7.1 \\
\hline & 20.3 & 25.08 & 7.1 \\
\hline & 96 & 20.0649 & \\
\hline g & 386 & 25.1202 & 5028 \\
\hline & 28 & 27.6 & \\
\hline g & 60 & 30.1 & 7.2 \\
\hline & 23.2 & 15.0 & 7.2 \\
\hline g & 18.8 & 27.5 & 7.2 \\
\hline & 20.3 & 30.1 & 7060 \\
\hline g & 20.3 & & 7060 \\
\hline & 32 & 30.1 & 7.2 \\
\hline g & 17.4 & 15.0 & 322 \\
\hline & 17.4 & 25.0 & 074042 \\
\hline $\mathrm{Ag}$ & 29 & 27.622200 & 7.21 \\
\hline & 29.0 & 30.11 & $7.21<$ \\
\hline g & 29.0 & 15.0 & 7.21 \\
\hline Ag & & 30.1100493122 & 7.2149251212 \\
\hline & 14.50 & 15.0340493122 & 7.214925121 \\
\hline $\mathrm{Ag}$ & 15.9 & 27.59653 & 7.21 \\
\hline & 15.9273390239 & 22.5639424327 & 7.2161786018 \\
\hline $\mathrm{Ag}$ & 365920 & 17.5458990974 & 7.21 \\
\hline $\mathrm{Ag}$ & 18.7940093666 & 22.5338014952 & 7.2175486624 \\
\hline $\mathrm{Ag}$ & 57406889 & 20.0272896293 & 7.2176006948 \\
\hline Ag & 18.8456237423 & 17.5327808017 & 7.2215840349 \\
\hline $\mathrm{Ag}$ & 26.1 & 25.0932694720 & 7.2218326 \\
\hline $\mathrm{Ag}$ & 26.1154039635 & 30.1253071195 & 7.2220715177 \\
\hline Ag & 26.1154039635 & 15.0493071195 & 7.2220715177 \\
\hline $\mathrm{Ag}$ & 27.5718740997 & 27.6044528794 & 7.2308200418 \\
\hline & & 17.5611429225 & 7.2357373938 \\
\hline$A \xi$ & 26.1057963254 & 20.0610823271 & 7.236476175 \\
\hline
\end{tabular}




$\begin{array}{llll}\mathrm{Ag} & 15.9418443262 & 17.5379637696 & 7.2376164467 \\ \mathrm{Ag} & 29.0138799679 & 25.0859211538 & 7.2414137617 \\ \mathrm{Ag} & 14.5078799679 & 25.0859211538 & 7.2414137617 \\ \mathrm{Ag} & 28.9945564651 & 20.0554576877 & 7.2416154397 \\ \mathrm{Ag} & 14.4885564651 & 20.0554576877 & 7.2416154397 \\ \mathrm{Ag} & 27.5553812385 & 17.5509984998 & 7.2430807902 \\ \mathrm{Ag} & 27.5575065977 & 22.5722367185 & 7.2526695482\end{array}$

Zwitterion on Au (114 Atom Cluster, SP calc from VASP)-ADF 130

$\begin{array}{llll}\mathrm{O} & 19.5661264406 & 23.1391270100 & 10.0980409932 \\ \mathrm{O} & 21.9897212834 & 27.2388542775 & 10.0522155077 \\ \mathrm{~N} & 21.8309738806 & 21.7700176573 & 10.1822438347 \\ \mathrm{~N} & 24.2720803996 & 25.9198782312 & 10.2325148073 \\ \mathrm{C} & 20.6594366617 & 25.2561535471 & 9.8546077762 \\ \mathrm{C} & 21.9454922993 & 23.0975116359 & 10.1944036298 \\ \mathrm{C} & 20.6070609978 & 23.8356503264 & 10.0713089034 \\ \mathrm{C} & 23.1670382486 & 25.1757135255 & 10.2145030274 \\ \mathrm{C} & 23.1660886520 & 23.7771828699 & 10.2738965068 \\ \mathrm{C} & 21.8787534607 & 25.9887209445 & 10.0563382215 \\ \mathrm{H} & 19.7227712377 & 25.8126458323 & 9.9210072556 \\ \mathrm{H} & 24.1058093245 & 23.2239917413 & 10.3391904894 \\ \mathrm{H} & 24.1468427845 & 26.9219481886 & 10.0698676040 \\ \mathrm{H} & 25.1978601973 & 25.5160275363 & 10.1275343460 \\ \mathrm{H} & 22.6335593747 & 21.1624532423 & 10.0454960254 \\ \mathrm{H} & 20.8933236931 & 21.3948329651 & 10.0206119345 \\ \mathrm{Au} & 14.6123311555 & 27.0250131369 & 4.8257472720 \\ \mathrm{Au} & 14.6101844500 & 16.8475039485 & 4.7934292016 \\ \mathrm{Au} & 14.5932437662 & 20.2972621684 & 7.2902831728 \\ \mathrm{Au} & 14.6254353039 & 30.3736703569 & 7.2690030935 \\ \mathrm{Au} & 14.6101844500 & 32.0535039485 & 4.7934292016 \\ \mathrm{Au} & 14.6320000000 & 28.7212752720 & 2.4232800000 \\ \mathrm{Au} & 17.5583122080 & 28.7212752720 & 2.4232800000 \\ \mathrm{Au} & 20.4846097840 & 28.7212752720 & 2.4232800000 \\ \mathrm{Au} & 14.6320000000 & 23.6530546480 & 2.4232800000 \\ \mathrm{Au} & 17.5583122080 & 23.6530546480 & 2.4232800000 \\ \mathrm{Au} & 16.0951561040 & 26.1871649600 & 2.4232800000 \\ \mathrm{Au} & 29.2443311555 & 27.0250131369 & 4.8257472720 \\ \mathrm{Au} & 17.5528189963 & 27.0484874491 & 4.7926693754 \\ \mathrm{Au} & 16.0811330489 & 29.5576770079 & 4.8034951715 \\ \mathrm{Au} & 14.6320000000 & 18.5848188180 & 2.4232800000 \\ \mathrm{Au} & 14.6474365043 & 21.9683800761 & 4.8212228055 \\ \mathrm{Au} & 14.6465089330 & 25.3223565313 & 7.4062457777 \\ \mathrm{Au} & 23.4109219920 & 28.7212752720 & 2.4232800000 \\ \mathrm{Au} & 26.3372195680 & 28.7212752720 & 2.4232800000 \\ \mathrm{Au} & 20.4846097840 & 23.6530546480 & 2.4232800000\end{array}$




\begin{tabular}{|c|c|c|c|}
\hline & 3.4109219920 & .6530546480 & \\
\hline $\mathrm{u}$ & 1.9477658880 & .1871649600 & 2.4232800000 \\
\hline $\mathrm{u}$ & 0.4838360385 & 27.0333410695 & 4.863292693 \\
\hline $\mathrm{Au}$ & 3.4096287514 & 27.0601653384 & 4.7547003596 \\
\hline & 1.9255988533 & 29.6135621741 & 4.7917532804 \\
\hline$A$ & 7.5583122080 & 8.5848188180 & 00000 \\
\hline & 0.4846097840 & 8.5848188180 & 2.4232800000 \\
\hline & 19.0214683120 & 118970 & ) \\
\hline & 17.5250564680 & 1.9834174694 & $4.79^{\prime}$ \\
\hline & 20.4476650329 & 1.95130 & 4.76 \\
\hline & 19.0 & 300 & 4.86 \\
\hline & 98950006 & 25.29963 & 7.30 \\
\hline & 3896 & 25.2513 & \\
\hline & 3120 & 26.1871 & 2.42 \\
\hline & 19.0 & 27.83681 & 7.26 \\
\hline & 19.0 & 29.58935 & 4.79 \\
\hline & 16.0 & 16.0 & 2.42 \\
\hline & 17. & 16.9 & 4.80 \\
\hline & 16.0 & 19.4226 & 4.80 \\
\hline & 29.2 & 20.2 & 7.29 \\
\hline$A$ & 17.5 & 20.18 & 7.29 \\
\hline & & & 2.42 \\
\hline & 16.0 & 22.80 & 91639 \\
\hline & 16.1 & $24.5247 €$ & 4.81 \\
\hline & 16 & 27.8 & 7.3 \\
\hline & 39 & 30.37367 & 7.2 \\
\hline$A$ & 26 & 23. & 2.4 \\
\hline & 27.8 & 26.1 & 00000 \\
\hline$A$ & 26.3 & 26.9 & 37563 \\
\hline & 27.7 & 29.50273 & 4.81 \\
\hline $\mathrm{u}$ & 23.4 & 18.58481 & 0000 \\
\hline & 5680 & 18.58481 & 00000 \\
\hline & 24.8 & 21.11892 & 00000 \\
\hline & 23.5 & 21.92116 & $4.76^{\prime}$ \\
\hline 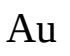 & 26.4 & $21.91556 \mathrm{~S}$ & 4.85 \\
\hline & & $24.41500 €$ & 4.7766624663 \\
\hline $\mathrm{Au}$ & 23.5 & 25.342771 & 7.0480526956 \\
\hline $\mathrm{Al}$ & 26.3 & 25.33125 & 25501 \\
\hline & $24.8^{\prime}$ & 26.187164 & 2.4232800000 \\
\hline $\mathrm{Al}$ & 020121 & 27.887334 & 753274 \\
\hline & 24.8 & 29.5615143368 & 4.8141411655 \\
\hline $\mathrm{Al}$ & 658880 & 16.050708 & 2.4232800000 \\
\hline & 32655754 & 16.9546867721 & 4.8213666012 \\
\hline 180 & & 16.920109 & 4.8186240201 \\
\hline $\mathrm{Al}$ & 21.9901638043 & 19.4656828076 & 4.8110902332 \\
\hline & & 20.1765112363 & 7.279 \\
\hline $\mathrm{Au}$ & 23.4298820537 & 20.1981123025 & 7.3659151085 \\
\hline
\end{tabular}




\begin{tabular}{|c|c|c|c|}
\hline $\mathrm{u}$ & 1.9477658880 & 21.1189291300 & \\
\hline $\mathrm{Au}$ & 1.9943743790 & 22.6844136457 & 7.0346915472 \\
\hline $\mathrm{u}$ & 1.9461939509 & 24.4989605708 & 4.7624816913 \\
\hline $\mathrm{Au}$ & 22.0225663807 & 27.9538840183 & 73048544326 \\
\hline & 7.5379828036 & 30.3728170659 & 7.2720560 \\
\hline $\mathrm{u}$ & 0.4652937981 & 617 & 77 \\
\hline & 9.0214683120 & 16.0507085060 & 2.423280000 \\
\hline & 19.0015088937 & 17.6869886981 & 73 \\
\hline & 19.0080747024 & 9.4377977927 & $4.80^{\circ}$ \\
\hline & 39326281 & 22.6637957416 & 7.2 \\
\hline & 16.0 & 17.704 & \\
\hline & 27.8003756720 & $16.0507 \mathrm{C}$ & 2.4 \\
\hline & & 524 & \\
\hline & 3090 & 0774 & 4.8 \\
\hline & 757 & 575 & \\
\hline & 756720 & 21.1189 & 0000 \\
\hline $\mathrm{A}$ & 555 & 22.8245 & \\
\hline $\mathrm{u}$ & 27. & 701 & \\
\hline & 037669 & 27.8 & \\
\hline & 00 & 58 & \\
\hline & 24.8 & 060 & 2.4 \\
\hline & 24.9 & 749 & \\
\hline & 24.9 & 951 & 4.8 \\
\hline & 248 & 122 & \\
\hline A & 21 & 77 & \\
\hline & & 17.757380 & 7.2 \\
\hline$A$ & 40 & 60 & 2.4 \\
\hline & 29.2 & 32.0535 & 4.7 \\
\hline & 17. & 956 & 2857 \\
\hline & 21.9 & 31.2567 & 2.42 \\
\hline $\mathrm{u}$ & 5754 & 32.1606 & 4.8 \\
\hline & 225 & 32.1261 & 4.818 \\
\hline & 4683120 & 31.2567 & 2.423 \\
\hline & 19.0 & 32.8929 & 7.32 \\
\hline . & 16.0 & 789 & 7.2 \\
\hline & 27.8 & 60 & 800000 \\
\hline $\mathrm{Au}$ & & 32.0537279524 & 4.8148660472 \\
\hline $\mathrm{A}$ & 23.4 & 30.4 & 66381 \\
\hline & 634640 & 31.2567085060 & 2.4232800000 \\
\hline $\mathrm{Al}$ & 7762827 & 32.9440 & 5506917 \\
\hline & 93142890 & 32.8937714577 & 7.3478392053 \\
\hline $\mathrm{Al}$ & 3469694 & 32.96338 & 7.2852160612 \\
\hline & 0000000 & 28.7212752720 & 2.4232800000 \\
\hline & & 23.6530 & 2.4232800000 \\
\hline & 29.2640000000 & 18.5848188180 & 2.4232800000 \\
\hline & & & 4.82 \\
\hline $\mathrm{Al}$ & 29.2785089330 & 25.3223565313 & 7.406245777 \\
\hline
\end{tabular}


Zwitterion on Cu (114 Atom Cluster, SP calc from VASP)-ADF 130

$\begin{array}{llll}\mathrm{O} & 21.2940902142 & 22.2336064088 & 8.7427028221 \\ \mathrm{O} & 16.4378791682 & 22.3519183671 & 8.7232716304 \\ \mathrm{~N} & 21.2678135563 & 19.5011009678 & 8.7230670294 \\ \mathrm{~N} & 16.3284322191 & 19.6067915897 & 8.7337625433 \\ \mathrm{C} & 20.1199406804 & 21.6421986350 & 8.8315213211 \\ \mathrm{C} & 18.7953315757 & 19.5242297151 & 8.9506495079 \\ \mathrm{C} & 20.0387442563 & 20.1941258267 & 8.8971906848 \\ \mathrm{C} & 17.5798937401 & 21.7013151725 & 8.8270844818 \\ \mathrm{C} & 17.5857159883 & 20.2530536276 & 8.9101593573 \\ \mathrm{C} & 18.8675667614 & 22.3531822728 & 8.8000301893 \\ \mathrm{H} & 15.5522262825 & 20.2006087754 & 9.0338521448 \\ \mathrm{H} & 16.2624099171 & 18.6571126178 & 9.0928778917 \\ \mathrm{H} & 21.2980225594 & 18.5427220806 & 9.0602675669 \\ \mathrm{H} & 22.0649005822 & 20.0614364787 & 9.0316522570 \\ \mathrm{H} & 18.7700395045 & 18.4317687626 & 9.0005169984 \\ \mathrm{H} & 18.8945762397 & 23.4432912581 & 8.8807069716 \\ \mathrm{Cu} & 12.5194347628 & 21.6972446105 & 6.5690050171 \\ \mathrm{Cu} & 12.5238426656 & 17.3527392231 & 6.5580362045 \\ \mathrm{Cu} & 12.5218547162 & 23.1387024201 & 4.4709309416 \\ \mathrm{Cu} & 12.5218409649 & 18.8002856646 & 4.4621626420 \\ \mathrm{Cu} & 12.5237278669 & 14.4600415852 & 4.4710516116 \\ \mathrm{Cu} & 12.5237736663 & 26.0385240918 & 6.5585886222 \\ \mathrm{Cu} & 12.5237278669 & 27.4760415852 & 4.4710516116 \\ \mathrm{Cu} & 25.0434347628 & 21.6972446105 & 6.5690050171 \\ \mathrm{Cu} & 15.0095367452 & 21.7020132069 & 6.5848725406 \\ \mathrm{Cu} & 23.8132289372 & 19.5217543736 & 6.5465883655 \\ \mathrm{Cu} & 22.5470663814 & 21.7056587763 & 6.6303845185 \\ \mathrm{Cu} & 13.7549794349 & 19.5213046939 & 6.5481349277 \\ \mathrm{Cu} & 20.0335780135 & 17.3159888269 & 6.5204898868 \\ \mathrm{Cu} & 17.5336375970 & 17.3225191482 & 6.5281730789 \\ \mathrm{Cu} & 18.7839741854 & 15.1761468991 & 6.5605394960 \\ \mathrm{Cu} & 13.7644883157 & 23.8737713477 & 6.5534926907 \\ \mathrm{Cu} & 23.8071385002 & 23.8747557201 & 6.5534441401 \\ \mathrm{Cu} & 22.5548887594 & 17.3307002044 & 6.5400001344 \\ \mathrm{Cu} & 15.0178062645 & 17.3354801877 & 6.5453924003 \\ \mathrm{Cu} & 21.2924129369 & 15.1815865139 & 6.5601078343 \\ \mathrm{Cu} & 16.2778845263 & 15.1823949233 & 6.5612856771 \\ \mathrm{Cu} & 16.2762332041 & 23.8740479141 & 6.6283950185 \\ \mathrm{Cu} & 21.2969986615 & 23.8836504858 & 6.5947303101 \\ \mathrm{Cu} & 25.0478426656 & 17.3527392231 & 6.5580362045 \\ \mathrm{Cu} & 23.7995854745 & 15.1839368156 & 6.5595113690 \\ \mathrm{Cu} & 13.7727370425 & 15.1844057140 & 6.5595214207 \\ \mathrm{Cu} & 18.7888465586 & 23.8901042566 & 6.4819671259\end{array}$




\begin{tabular}{|c|c|c|c|}
\hline & 18.7856026570 & 19.4931521961 & 6.4 \\
\hline & 21.3322304223 & 19.4817090017 & 5591073203 \\
\hline & 16.2392968694 & 19.4954813757 & 5717129533 \\
\hline $\mathrm{Cu}$ & 17.5395241628 & 21.7100686112 & 6.4244607730 \\
\hline & 20.0173115056 & 21.7113314880 & .4326327611 \\
\hline $\mathrm{u}$ & 13.7770821366 & 20.9750385790 & 83165 \\
\hline $\mathrm{u}$ & 23.7846762003 & 20.9776613770 & 4.48902 \\
\hline $\mathrm{Cu}$ & 18.7837539413 & 16.6273026286 & 4.4551 \\
\hline $\mathrm{Cu}$ & 25.0458547162 & 23.1387024201 & \\
\hline 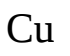 & 15.0301195125 & 5619072 & 4.4 \\
\hline $\mathrm{u}$ & 25.045 & 2856646 & \\
\hline $\mathrm{Cu}$ & $22.540^{\prime}$ & 23.1355559782 & 4.4 \\
\hline $\mathrm{Cu}$ & 21.29 & 711685 & \\
\hline o & 30856 & 971402 & 4.4 \\
\hline $\mathrm{u}$ & 1862 & 210 & \\
\hline $\mathrm{Cu}$ & 20.039 & 008568 & 4.469 \\
\hline C & 23.7 & 25.3 & \\
\hline $\mathrm{Cu}$ & $13.7^{\prime}$ & 25. & 4.4 \\
\hline & 00090 & 190656 & 313 \\
\hline u & 21.2 & 25. & 4.4 \\
\hline 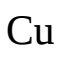 & 25.047 & 14. & 4.4 \\
\hline $\mathrm{Cu}$ & 15.0 & 14. & \\
\hline 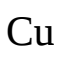 & 6664 & 14. & 642 \\
\hline 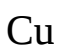 & 18.783 & 324203 & \\
\hline $\mathrm{Cu}$ & 13.7 & 16. & 4.4 \\
\hline & & & \\
\hline $\mathrm{u}$ & 20.0 & 18. & 742 \\
\hline & 17.5148126864 & 18. & 131 \\
\hline $\mathrm{Cu}$ & 2049 & 18. & 126 \\
\hline & 47954 & 18. & 748 \\
\hline $\mathrm{Cu}$ & 16.265 & 26925 & 4.4 \\
\hline & 21.302 & 20. & 4.4908 \\
\hline $\mathrm{Cu}$ & 20.050 & 138362 & 4.4 \\
\hline $\mathrm{Cu}$ & 05506 & 446490 & 05056018 \\
\hline $\mathrm{Cu}$ & 1918 & 20. & 4.43609 \\
\hline $\mathrm{Cu}$ & 68040 & 18. & 2.4232800000 \\
\hline$C_{u}$ & 18.7857996160 & 366800 & 2.4232800000 \\
\hline $\mathrm{Cu}$ & 19480 & 345600 & 2.4232800000 \\
\hline$\Omega_{0}$ & 21.2905119480 & 3366800 & 2.4232800000 \\
\hline $\mathrm{Cu}$ & 22.5428743760 & 942480 & 2.4232800000 \\
\hline$C_{1}$ & 20.0381620440 & 15.9081942480 & 2.4232800000 \\
\hline $\mathrm{Cu}$ & 23.7952368040 & 6345600 & 2.4232800000 \\
\hline$C_{1}$ & 22.5428743760 & 20.2464921280 & 2.4232800000 \\
\hline $\mathrm{Cu}$ & 13.7763624280 & 18.0773366800 & 2.4232800000 \\
\hline $\mathrm{Cu}$ & 16.2810872840 & 22.4156345600 & 2.4232800000 \\
\hline $\mathrm{Cu}$ & 16.2810 & 18.0773366800 & 2.4 \\
\hline $\mathrm{Cu}$ & 17.5334371880 & 15.9081942480 & 2.4232800000 \\
\hline
\end{tabular}




\begin{tabular}{|c|c|c|c|}
\hline $\mathrm{Cu}$ & 15.0287248560 & 15.9081942480 & 2.4232800000 \\
\hline & 18.7857996160 & 22.4156345600 & 2.4232800000 \\
\hline & 20.0381620440 & 20.2464921280 & .4232800000 \\
\hline $\mathrm{Cu}$ & 17.5334371880 & 20.2464921280 & 2.4232800000 \\
\hline & 22.5428743760 & 24.5847769920 & 2.4232800000 \\
\hline $\mathrm{Cu}$ & 20.0381620440 & 24.5847769920 & 2.4232800000 \\
\hline $\mathrm{Cu}$ & 12.5240000000 & 15.9081942480 & 2.4232800000 \\
\hline $\mathrm{Cu}$ & 13.7763624280 & 22.4156345600 & 300000 \\
\hline $\mathrm{Cu}$ & 15.0287248560 & 20.2464921280 & 2.4232800000 \\
\hline $\mathrm{Cu}$ & 12.5240000000 & 20.24649 & 2.4 \\
\hline 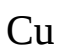 & 17.5334371880 & 24.5 & 2.4 \\
\hline 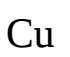 & 15.0287248560 & 24.5847769920 & 00000 \\
\hline $\mathrm{Cu}$ & 12.5240000000 & 24.58477 & 2.4 \\
\hline o & 17.5326328320 & 26.03671 & 6.5 \\
\hline $\mathrm{Cu}$ & 20.0379836308 & 26.0386 & \\
\hline $\mathrm{Cu}$ & 25.0477736663 & 26.03852 & 6.5 \\
\hline $\mathrm{Cu}$ & 22.54 & 26.03 & \\
\hline $\mathrm{Cu}$ & 15.02 & 26.0 & 6.5 \\
\hline $\mathrm{Cu}$ & & & 4.4 \\
\hline$u$ & 20.039058 & 27.4 & 4.4 \\
\hline $\mathrm{Cu}$ & 25.0477278669 & 27.4760 & 4.4 \\
\hline $\mathrm{Cu}$ & 15.0 & 27.4 & 4.4 \\
\hline $\mathrm{Cu}$ & 22.54 & 27.47689 & 4.4 \\
\hline $\mathrm{Cu}$ & 21.2905119480 & 26.75505 & 0000 \\
\hline $\mathrm{Cu}$ & 23.7952368040 & 26.75505 & 300000 \\
\hline $\mathrm{Cu}$ & 16.2810872840 & 26.7550 & 2.4 \\
\hline $\mathrm{Cu}$ & 18.7857996160 & 26.75505 & 00000 \\
\hline $\mathrm{Cu}$ & 13.7763624280 & 26.75505 & 00000 \\
\hline $\mathrm{Cu}$ & 26.278979 & 19.5213 & 49277 \\
\hline$C_{U}$ & 26.2884883157 & 23.87377 & 26907 \\
\hline $\mathrm{Cu}$ & 26.2967370425 & 15.18440 & 14207 \\
\hline$C_{11}$ & 26.3010821366 & 20.9750385790 & 4.48 \\
\hline $\mathrm{Cu}$ & 26.2988732900 & 25.30885 & 320468 \\
\hline$C_{1}$ & 26.2959094628 & 16.6294757144 & 4.4677078345 \\
\hline $\mathrm{Cu}$ & 26.3003624280 & 18.07733 & 800000 \\
\hline $\mathrm{Cu}$ & 25.0480000000 & 15.9081942480 & 2.4232800000 \\
\hline $\mathrm{Cu}$ & 26.3003624280 & 22.4156345600 & 2.4232800000 \\
\hline $\mathrm{Cu}$ & 25.0480000000 & 20.2464921280 & 2.4232800000 \\
\hline $\mathrm{Cl}$ & 25.0 & 24.58 & 0000 \\
\hline $\mathrm{Cu}$ & 26.3003624280 & 26.7550518160 & 80000 \\
\hline
\end{tabular}

Zwitterion on $\mathrm{Cu}$ - VASP -DFT-D2

$\mathrm{Cu} \mathrm{O} \mathrm{N} \mathrm{C} \mathrm{H}$

1.00000000000000

$\begin{array}{llll}12.7810000000000006 & 0.0000000000000000 & 0.0000000000000000\end{array}$

$\begin{array}{llll}6.3905000000000003 & 11.06866999999999991 & 0.0000000000000000\end{array}$

$\begin{array}{llll}0.0000000000000000 & 0.0000000000000000 & 40.0000000000000000\end{array}$ 
100

Direct

$0.20000000000000280 .0000000000000000 \quad 0.0000000000000000$

$\begin{array}{llll}0.0000000000000000 & 0.2000000000000028 & 0.0000000000000000\end{array}$

$\begin{array}{lll}0.0000000000000000 & 0.0000000000000000 & 0.0000000000000000\end{array}$

$\begin{array}{llll}0.5999899999999982 & 0.0000000000000000 & 0.0000000000000000\end{array}$

$\begin{array}{lllll}0.2000000000000028 & 0.2000000000000028 & 0.0000000000000000\end{array}$

$\begin{array}{llll}0.3999900000000025 & 0.2000000000000028 & 0.0000000000000000\end{array}$

0.20000000000000280 .39999000000000250 .0000000000000000

0.39999000000000250 .00000000000000000 .0000000000000000

$\begin{array}{llll}0.0000000000000000 & 0.5999899999999982 & 0.0000000000000000\end{array}$

$\begin{array}{llll}0.0000000000000000 & 0.3999900000000025 & 0.0000000000000000\end{array}$

$\begin{array}{llll}0.5999899999999982 & 0.2000000000000028 & 0.0000000000000000\end{array}$

$\begin{array}{llll}0.7999900000000011 & 0.2000000000000028 & 0.0000000000000000\end{array}$

$\begin{array}{lll}0.5999899999999982 & 0.3999900000000025 & 0.0000000000000000\end{array}$

$\begin{array}{llll}0.7999900000000011 & 0.0000000000000000 & 0.0000000000000000\end{array}$

$\begin{array}{llll}0.2000000000000028 & 0.5999899999999982 & 0.0000000000000000\end{array}$

0.39999000000000250 .59998999999999820 .0000000000000000

0.20000000000000280 .79999000000000110 .0000000000000000

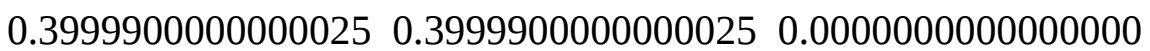

$\begin{array}{llll}0.0000000000000000 & 0.7999900000000011 & 0.0000000000000000\end{array}$

$\begin{array}{llll}0.5999899999999982 & 0.5999899999999982 & 0.0000000000000000\end{array}$

$\begin{array}{lll}0.7999900000000011 & 0.5999899999999982 & 0.0000000000000000\end{array}$

$\begin{array}{lll}0.5999899999999982 & 0.7999900000000011 & 0.0000000000000000\end{array}$

$\begin{array}{lll}0.7999900000000011 & 0.3999900000000025 & 0.0000000000000000\end{array}$

$\begin{array}{llll}0.3999900000000025 & 0.7999900000000011 & 0.0000000000000000\end{array}$

$\begin{array}{lllll}0.7999900000000011 & 0.7999900000000011 & 0.0000000000000000\end{array}$

$\begin{array}{llll}0.3333299999999966 & 0.1333300000000008 & 0.0521799999999999\end{array}$

$\begin{array}{llll}0.1333300000000008 & 0.3333299999999966 & 0.0521799999999999\end{array}$

$\begin{array}{llll}0.1333300000000008 & 0.1333300000000008 & 0.0521799999999999\end{array}$

$\begin{array}{llll}0.7333199999999991 & 0.1333300000000008 & 0.0521799999999999\end{array}$

$\begin{array}{lllll}0.3333299999999966 & 0.3333299999999966 & 0.0521799999999999\end{array}$

$\begin{array}{llll}0.5333299999999994 & 0.3333299999999966 & 0.0521799999999999\end{array}$

$\begin{array}{llll}0.3333299999999966 & 0.5333299999999994 & 0.0521799999999999\end{array}$

$\begin{array}{llll}0.5333299999999994 & 0.1333300000000008 & 0.0521799999999999\end{array}$

$\begin{array}{llll}0.1333300000000008 & 0.7333199999999991 & 0.0521799999999999\end{array}$

$\begin{array}{llll}0.1333300000000008 & 0.5333299999999994 & 0.0521799999999999\end{array}$

$\begin{array}{llll}0.7333199999999991 & 0.3333299999999966 & 0.0521799999999999\end{array}$

$\begin{array}{lllll}0.9333200000000019 & 0.3333299999999966 & 0.0521799999999999\end{array}$

$\begin{array}{llll}0.7333199999999991 & 0.5333299999999994 & 0.0521799999999999\end{array}$

$\begin{array}{llll}0.9333200000000019 & 0.1333300000000008 & 0.0521799999999999\end{array}$

$\begin{array}{llll}0.3333299999999966 & 0.7333199999999991 & 0.0521799999999999\end{array}$

$\begin{array}{llll}0.5333299999999994 & 0.7333199999999991 & 0.0521799999999999\end{array}$

$\begin{array}{llll}0.3333299999999966 & 0.9333200000000019 & 0.0521799999999999\end{array}$

$\begin{array}{llll}0.5333299999999994 & 0.5333299999999994 & 0.0521799999999999\end{array}$

$\begin{array}{llll}0.1333300000000008 & 0.9333200000000019 & 0.0521799999999999\end{array}$

$\begin{array}{llll}0.7333199999999991 & 0.7333199999999991 & 0.0521799999999999\end{array}$ 
$\begin{array}{llll}0.9333200000000019 & 0.7333199999999991 & 0.0521799999999999\end{array}$

$\begin{array}{llll}0.7333199999999991 & 0.9333200000000019 & 0.0521799999999999\end{array}$

$\begin{array}{llll}0.9333200000000019 & 0.5333299999999994 & 0.0521799999999999\end{array}$

0.53332999999999940 .93332000000000190 .0521799999999999

$\begin{array}{llll}0.9333200000000019 & 0.9333200000000019 & 0.0521799999999999\end{array}$

$\begin{array}{llll}0.0656913547370275 & 0.0661161100214372 & 0.1031309987735389\end{array}$

$\begin{array}{llll}0.2662273022397921 & 0.0654645260696909 & 0.1029805429645809\end{array}$

$\begin{array}{llll}0.0654997086551950 & 0.2659777832310690 & 0.1030678017623990\end{array}$

$\begin{array}{llll}0.4654908329369220 & 0.0675295620847927 & 0.1035984995215115\end{array}$

$\begin{array}{llll}0.6666230702577084 & 0.0658609448581234 & 0.1034400975854866\end{array}$

$\begin{array}{llll}0.4678986027930757 & 0.2633663385559563 & 0.1035575363215047\end{array}$

$\begin{array}{llll}0.0678440683444685 & 0.4642961640528344 & 0.1041236496081466\end{array}$

$\begin{array}{llll}0.2641836381185768 & 0.4651812091797932 & 0.1024395346026919\end{array}$

$\begin{array}{llll}0.0653639448186278 & 0.6658895689225225 & 0.1030501109008319\end{array}$

$\begin{array}{llll}0.2660791038063448 & 0.2664806817871638 & 0.1039103839901725\end{array}$

$\begin{array}{llll}0.8670378928873729 & 0.0650698836097317 & 0.1030084492104647\end{array}$

$\begin{array}{llll}0.8654398106148080 & 0.2664311744472496 & 0.1029226456721462\end{array}$

$\begin{array}{llll}0.4677112296674827 & 0.4653416937812713 & 0.1018568681899854\end{array}$

$\begin{array}{llll}0.6652148401111702 & 0.4681254044478704 & 0.1039982249366200\end{array}$

$\begin{array}{llll}0.4681027335999075 & 0.6666972303328567 & 0.1031223141352130\end{array}$

$\begin{array}{llll}0.6656886143616417 & 0.2644792828322804 & 0.1037537149679390\end{array}$

$\begin{array}{llll}0.0687470859800880 & 0.8644996662685026 & 0.1044850128087400\end{array}$

0.26502999934543220 .86477748036752950 .1037760034395197

$\begin{array}{llll}0.2639356689982185 & 0.6676412515080430 & 0.1028800289830263\end{array}$

$\begin{array}{llll}0.8661104746136276 & 0.4661816557799326 & 0.1029909974004091\end{array}$

$\begin{array}{llll}0.8658334876169772 & 0.6655806176412078 & 0.1029066736652171\end{array}$

$\begin{array}{llll}0.4665902518176757 & 0.8667453177120282 & 0.1028245420359142\end{array}$

$\begin{array}{llll}0.6668770781223741 & 0.8657645692225430 & 0.1027506943926256\end{array}$

$\begin{array}{llll}0.6655974953856029 & 0.6656186708923337 & 0.1038360003101983\end{array}$

$\begin{array}{llll}0.8666687387464179 & 0.8656129573891373 & 0.1028952935005561\end{array}$

$\begin{array}{llll}0.1975906598780831 & 0.9999239008216214 & 0.1543755612883690\end{array}$

0.99784005292527290 .19863366911579550 .1545760356549095

0.99966016114481030 .99835463548054840 .1548256183638422

$\begin{array}{llll}0.5995853697753001 & 0.9960954729893564 & 0.1541735095560307\end{array}$

$\begin{array}{llll}0.1960436032516705 & 0.1964011606093057 & 0.1541059869369453\end{array}$

$\begin{array}{llll}0.3965761420519233 & 0.1968563276675480 & 0.1556329109765764\end{array}$

$\begin{array}{llll}0.1944492048262520 & 0.3962981374603535 & 0.1572590133224894\end{array}$

0.39840017115852120 .99940062014491640 .1546758223463207

0.99695057580414550 .59893954455924840 .1544279838894539

$\begin{array}{llll}0.9969400626574484 & 0.3981409638532439 & 0.1546514187139839\end{array}$

$\begin{array}{llll}0.6030429317100962 & 0.1909345082280090 & 0.1562088375490676\end{array}$

$\begin{array}{llll}0.8018154298555377 & 0.1968604074934737 & 0.1542003754485919\end{array}$

$\begin{array}{llll}0.6029832122429452 & 0.3962130875125458 & 0.1536801000197929\end{array}$

$\begin{array}{llll}0.8023077508725182 & 0.9959623661308653 & 0.1542835469790773\end{array}$

$\begin{array}{llll}0.1958056891515840 & 0.5974605043610793 & 0.1530547004681533\end{array}$

$\begin{array}{llll}0.4004286827082074 & 0.5984278706923917 & 0.1505533383921331\end{array}$

$\begin{array}{llll}0.2000778762550085 & 0.7984357311254937 & 0.1592241659259303\end{array}$ 
$\begin{array}{lll}0.3988882587550061 & 0.4018863904729675 & 0.1503534367092119\end{array}$

$\begin{array}{llll}0.9988587722709313 & 0.7978895595782081 & 0.1545115499997854\end{array}$

$\begin{array}{llll}0.5994828438993572 & 0.6030146793811890 & 0.1577324346939770\end{array}$

$\begin{array}{llll}0.7996964066872200 & 0.5985145862840305 & 0.1545508600673543\end{array}$

$\begin{array}{llll}0.5995607411368196 & 0.8008495785818656 & 0.1543477851825277\end{array}$

$\begin{array}{llll}0.8000224596825669 & 0.3981839632338670 & 0.1544366399915780\end{array}$

$\begin{array}{lllll}0.3989850600760551 & 0.8038869979557406 & 0.1533294123390231\end{array}$

$\begin{array}{llll}0.7992961966329558 & 0.7984481207755861 & 0.1542999050389773\end{array}$

\section{Zwitterion on $\mathrm{Cu}$ - VASP -vdW-DF2 \\ $\mathrm{Cu} \mathrm{O} \mathrm{N} \mathrm{C} \mathrm{H}$ \\ 1.00000000000000 \\ $\begin{array}{llll}12.7810000000000006 & 0.0000000000000000 & 0.0000000000000000\end{array}$ \\ $\begin{array}{llll}6.3905000000000003 & 11.06866999999999991 & 0.0000000000000000\end{array}$ \\ $\begin{array}{llll}0.0000000000000000 & 0.0000000000000000 & 40.0000000000000000\end{array}$ \\ $\begin{array}{lllll}100 & 2 & 2 & 6 & 6\end{array}$}

Direct

$\begin{array}{llll}0.2000000000000028 & 0.0000000000000000 & 0.0000000000000000\end{array}$

$\begin{array}{lllll}0.0000000000000000 & 0.2000000000000028 & 0.0000000000000000\end{array}$

$\begin{array}{lllll}0.0000000000000000 & 0.0000000000000000 & 0.0000000000000000\end{array}$

$\begin{array}{lllll}0.5999899999999982 & 0.0000000000000000 & 0.0000000000000000\end{array}$

$\begin{array}{lllll}0.2000000000000028 & 0.2000000000000028 & 0.0000000000000000\end{array}$

$\begin{array}{lllll}0.3999900000000025 & 0.2000000000000028 & 0.0000000000000000\end{array}$

$\begin{array}{lllll}0.2000000000000028 & 0.3999900000000025 & 0.0000000000000000\end{array}$

$\begin{array}{llll}0.3999900000000025 & 0.0000000000000000 & 0.0000000000000000\end{array}$

$\begin{array}{lllll}0.0000000000000000 & 0.59998999999999982 & 0.0000000000000000\end{array}$

$\begin{array}{lllll}0.0000000000000000 & 0.3999900000000025 & 0.0000000000000000\end{array}$

$\begin{array}{llllll}0.5999899999999982 & 0.2000000000000028 & 0.0000000000000000\end{array}$

$\begin{array}{lllll}0.7999900000000011 & 0.2000000000000028 & 0.0000000000000000\end{array}$

$\begin{array}{lllll}0.5999899999999982 & 0.3999900000000025 & 0.0000000000000000\end{array}$

$\begin{array}{lllll}0.7999900000000011 & 0.0000000000000000 & 0.0000000000000000\end{array}$

$\begin{array}{lllll}0.2000000000000028 & 0.5999899999999982 & 0.0000000000000000\end{array}$

$\begin{array}{lllll}0.3999900000000025 & 0.5999899999999982 & 0.0000000000000000\end{array}$

$\begin{array}{lllll}0.2000000000000028 & 0.7999900000000011 & 0.0000000000000000\end{array}$

$\begin{array}{llll}0.3999900000000025 & 0.3999900000000025 & 0.0000000000000000\end{array}$

$\begin{array}{lllll}0.0000000000000000 & 0.7999900000000011 & 0.0000000000000000\end{array}$

$\begin{array}{lllll}0.5999899999999982 & 0.5999899999999982 & 0.0000000000000000\end{array}$

$\begin{array}{lllll}0.7999900000000011 & 0.5999899999999982 & 0.0000000000000000\end{array}$

$\begin{array}{lllll}0.5999899999999982 & 0.7999900000000011 & 0.0000000000000000\end{array}$

$\begin{array}{llll}0.7999900000000011 & 0.3999900000000025 & 0.0000000000000000\end{array}$

$\begin{array}{lllll}0.3999900000000025 & 0.7999900000000011 & 0.0000000000000000\end{array}$

$\begin{array}{lllll}0.7999900000000011 & 0.7999900000000011 & 0.0000000000000000\end{array}$

$\begin{array}{lllll}0.3333299999999966 & 0.1333300000000008 & 0.0521799999999999\end{array}$

$\begin{array}{lllll}0.1333300000000008 & 0.3333299999999966 & 0.0521799999999999\end{array}$

$\begin{array}{lllll}0.1333300000000008 & 0.1333300000000008 & 0.0521799999999999\end{array}$

$\begin{array}{llllll}0.7333199999999991 & 0.1333300000000008 & 0.0521799999999999\end{array}$

$\begin{array}{lllll}0.3333299999999966 & 0.3333299999999966 & 0.0521799999999999\end{array}$ 
$\begin{array}{llll}0.5333299999999994 & 0.3333299999999966 & 0.0521799999999999\end{array}$

$\begin{array}{llll}0.3333299999999966 & 0.5333299999999994 & 0.0521799999999999\end{array}$

$\begin{array}{llll}0.5333299999999994 & 0.1333300000000008 & 0.0521799999999999\end{array}$

$\begin{array}{llll}0.1333300000000008 & 0.7333199999999991 & 0.0521799999999999\end{array}$

$\begin{array}{llll}0.1333300000000008 & 0.5333299999999994 & 0.0521799999999999\end{array}$

$\begin{array}{llll}0.7333199999999991 & 0.3333299999999966 & 0.0521799999999999\end{array}$

$\begin{array}{llll}0.9333200000000019 & 0.3333299999999966 & 0.0521799999999999\end{array}$

$\begin{array}{llll}0.7333199999999991 & 0.5333299999999994 & 0.0521799999999999\end{array}$

$\begin{array}{llll}0.9333200000000019 & 0.1333300000000008 & 0.0521799999999999\end{array}$

$\begin{array}{llll}0.3333299999999966 & 0.7333199999999991 & 0.0521799999999999\end{array}$

$\begin{array}{llll}0.5333299999999994 & 0.7333199999999991 & 0.0521799999999999\end{array}$

$\begin{array}{llll}0.3333299999999966 & 0.9333200000000019 & 0.0521799999999999\end{array}$

0.53332999999999940 .53332999999999940 .0521799999999999

$\begin{array}{llll}0.1333300000000008 & 0.9333200000000019 & 0.0521799999999999\end{array}$

$\begin{array}{llll}0.7333199999999991 & 0.7333199999999991 & 0.0521799999999999\end{array}$

$\begin{array}{lllll}0.9333200000000019 & 0.7333199999999991 & 0.0521799999999999\end{array}$

$\begin{array}{llll}0.7333199999999991 & 0.9333200000000019 & 0.0521799999999999\end{array}$

$\begin{array}{llll}0.9333200000000019 & 0.5333299999999994 & 0.0521799999999999\end{array}$

0.53332999999999940 .93332000000000190 .0521799999999999

$\begin{array}{llll}0.9333200000000019 & 0.9333200000000019 & 0.0521799999999999\end{array}$

$\begin{array}{llll}0.0672686348746955 & 0.0649155716806440 & 0.1076308912645398\end{array}$

$\begin{array}{llll}0.2668556298946166 & 0.0651658145787578 & 0.1074465234341581\end{array}$

$\begin{array}{llll}0.0670042282630652 & 0.2652790192419018 & 0.1073715996403676\end{array}$

$\begin{array}{llll}0.4666070445189746 & 0.0658208506380896 & 0.1076763430479624\end{array}$

$\begin{array}{llll}0.6673009604149506 & 0.0658464018313909 & 0.1085062040246938\end{array}$

$\begin{array}{llll}0.4684959692887193 & 0.2630875281938422 & 0.1072580278248592\end{array}$

$\begin{array}{llll}0.0689339977881066 & 0.4642357095522993 & 0.1093073559578611\end{array}$

$0.26500485973537290 .4656871413532604 \quad 0.1067122403548735$

0.06595205447437150 .66654890720689290 .1072365759415235

$\begin{array}{llll}0.2665382614854570 & 0.2666291382287267 & 0.1088657593210428\end{array}$

$\begin{array}{llll}0.8680911772250051 & 0.0646651819507562 & 0.1075688407397658\end{array}$

$\begin{array}{llll}0.8674139811593160 & 0.2651092366728528 & 0.1073775873053921\end{array}$

$\begin{array}{llll}0.4679967339510218 & 0.4657660164486639 & 0.1056070607174545\end{array}$

$\begin{array}{llll}0.6683408131741619 & 0.4654738689311147 & 0.1069044399105514\end{array}$

$\begin{array}{llll}0.4675809504979430 & 0.6671384188155566 & 0.1065530037582921\end{array}$

$\begin{array}{llll}0.6670881939861548 & 0.2640675279670441 & 0.1083181435444113\end{array}$

$\begin{array}{llll}0.0685375907149278 & 0.8649471104672537 & 0.1087293803996613\end{array}$

$\begin{array}{llll}0.2664137247984010 & 0.8650391104001314 & 0.1079895997632213\end{array}$

0.26550277240521530 .66727954357793880 .1065786367934003

$\begin{array}{llll}0.8679062609605689 & 0.4654714791124816 & 0.1074224941086881\end{array}$

$\begin{array}{llll}0.8674994101285807 & 0.6653129121976793 & 0.1073621853401319\end{array}$

$\begin{array}{llll}0.4670760510870339 & 0.8660788332252347 & 0.1073595479740646\end{array}$

0.66751350483960990 .86570379099398590 .1072755745033634

$\begin{array}{llll}0.6679595661546265 & 0.6660788508947982 & 0.1072135090872051\end{array}$

$\begin{array}{llll}0.8673322040453296 & 0.8657476223822702 & 0.1074840346095320\end{array}$

$\begin{array}{llll}0.2003138964735085 & 0.9947089951091096 & 0.1633980020056225\end{array}$

$\begin{array}{llll}0.0002267550026951 & 0.1969031784451060 & 0.1634581975136101\end{array}$ 
$0.0039043952733403 \quad 0.99458251562386770 .1641393855153435$

$\begin{array}{llll}0.6015437676406030 & 0.9958597403686252 & 0.1630274503102216\end{array}$

$\begin{array}{llll}0.1989208338142575 & 0.1951022138986769 & 0.1626600908239611\end{array}$

$\begin{array}{llll}0.4009393658288111 & 0.1955882055739658 & 0.1628383445820934\end{array}$

$\begin{array}{llll}0.1997103000697296 & 0.3959607960781453 & 0.1699952993269117\end{array}$

$\begin{array}{llll}0.4002366368952631 & 0.9970901808015553 & 0.1634191893734922\end{array}$

$\begin{array}{llll}0.0007043470441023 & 0.5981488646351859 & 0.1631148343790267\end{array}$

$\begin{array}{llll}1.0002666791142509 & 0.3971863615148472 & 0.1635179455428820\end{array}$

$\begin{array}{llll}0.6022850015557889 & 0.1958960744526281 & 0.1672551269924839\end{array}$

$\begin{array}{llll}0.8023728091181191 & 0.1966976049004255 & 0.1630200850123441\end{array}$

$\begin{array}{llll}0.6018680495826839 & 0.3996455143652248 & 0.1612856346410274\end{array}$

$\begin{array}{llll}0.8026266033219692 & 0.9965719126472358 & 0.1634092258946604\end{array}$

$\begin{array}{llll}0.1993949860470812 & 0.6016630930411719 & 0.1603303491220586\end{array}$

$\begin{array}{llll}0.3988755425116609 & 0.6023857201489115 & 0.1584917285369783\end{array}$

$\begin{array}{llll}0.1998220401356512 & 0.8015022475850722 & 0.1836198723687778\end{array}$

$\begin{array}{llll}0.4014292321985174 & 0.4007518266534869 & 0.1571326067392878\end{array}$

$\begin{array}{llll}0.0031931538296987 & 0.7981650943429296 & 0.1635546376418206\end{array}$

$\begin{array}{llll}0.6010835284484173 & 0.5994880921193251 & 0.1622226919069844\end{array}$

$\begin{array}{llll}0.8009123213634601 & 0.5979465265464985 & 0.1635149230884739\end{array}$

$\begin{array}{llll}0.6009130403614839 & 0.7978498061381563 & 0.1634579536567624\end{array}$

$\begin{array}{llll}0.8008992117969332 & 0.3979794444663781 & 0.1633254112386188\end{array}$

$\begin{array}{llll}0.3991297355186887 & 0.7991685676647509 & 0.1624277606048571\end{array}$

$\begin{array}{llll}0.8017012644674015 & 0.7975616497715581 & 0.1633719734294529\end{array}$

$\begin{array}{llll}0.3130758209056088 & 0.3363603577789486 & 0.2144086421773596\end{array}$

$\begin{array}{llll}0.2769533395688372 & 0.7270788862602127 & 0.2281941259346748\end{array}$

$\begin{array}{llll}0.5586032157627960 & 0.2245760271210608 & 0.2264757581161979\end{array}$

$\begin{array}{llll}0.5166198027980415 & 0.6222848991028257 & 0.2441240000195553\end{array}$

$\begin{array}{llll}0.4859460735902808 & 0.3506636100386599 & 0.2318285555179279\end{array}$

$\begin{array}{llll}0.2879555379669937 & 0.5332421522691899 & 0.2261505188609292\end{array}$

$\begin{array}{llll}0.3584430175648448 & 0.4055488552846653 & 0.2244987508644792\end{array}$

$\begin{array}{llll}0.4679443536750119 & 0.5492923031730697 & 0.2385981908963260\end{array}$

$\begin{array}{llll}0.5378792711375786 & 0.4222588495528848 & 0.2387484220465668\end{array}$

$\begin{array}{llll}0.3389701096919697 & 0.6078434312498719 & 0.2314041826799962\end{array}$

$\begin{array}{llll}0.6345104783981435 & 0.3793034542828098 & 0.2432289119714234\end{array}$

$\begin{array}{llll}0.1908394876275770 & 0.5756479165565324 & 0.2231132861049614\end{array}$

$\begin{array}{llll}0.4623553284780255 & 0.7112034782863650 & 0.2389942985503699\end{array}$

$\begin{array}{llll}0.6064022743414696 & 0.5886498724748928 & 0.2412092733799004\end{array}$

$\begin{array}{llll}0.6401754599358106 & 0.1850603767408966 & 0.2380333243219241\end{array}$

$\begin{array}{llll}0.5137315627329987 & 0.1788964820720430 & 0.2306690520146444\end{array}$

Zwitterion on $\mathrm{Cu}$ - VASP - optB88-vdW - Start B

$\mathrm{Cu} \mathrm{O} \mathrm{N} \mathrm{C} \mathrm{H}$

1.00000000000000

$\begin{array}{lll}12.7810000000000006 & 0.0000000000000000 & 0.0000000000000000\end{array}$

$\begin{array}{lll}6.3905000000000003 & 11.0686699999999991 & 0.0000000000000000\end{array}$

$\begin{array}{llll}0.0000000000000000 & 0.0000000000000000 & 40.0000000000000000\end{array}$

$\begin{array}{lllll}100 & 2 & 2 & 6 & 6\end{array}$ 
Direct

$\begin{array}{llll}0.2000000000000028 & 0.0000000000000000 & 0.0000000000000000\end{array}$

$\begin{array}{llll}0.0000000000000000 & 0.2000000000000028 & 0.0000000000000000\end{array}$

$\begin{array}{lll}0.0000000000000000 & 0.0000000000000000 & 0.0000000000000000\end{array}$

0.59998999999999820 .00000000000000000 .0000000000000000

$\begin{array}{llll}0.2000000000000028 & 0.2000000000000028 & 0.0000000000000000\end{array}$

$\begin{array}{llll}0.3999900000000025 & 0.2000000000000028 & 0.0000000000000000\end{array}$

0.20000000000000280 .39999000000000250 .0000000000000000

0.39999000000000250 .00000000000000000 .0000000000000000

$\begin{array}{lll}0.0000000000000000 & 0.5999899999999982 & 0.0000000000000000\end{array}$

$\begin{array}{lll}0.0000000000000000 & 0.3999900000000025 & 0.0000000000000000\end{array}$

$\begin{array}{llll}0.5999899999999982 & 0.2000000000000028 & 0.0000000000000000\end{array}$

$\begin{array}{llll}0.7999900000000011 & 0.2000000000000028 & 0.0000000000000000\end{array}$

$\begin{array}{lll}0.5999899999999982 & 0.3999900000000025 & 0.0000000000000000\end{array}$

$\begin{array}{lll}0.7999900000000011 & 0.0000000000000000 & 0.0000000000000000\end{array}$

$\begin{array}{llll}0.2000000000000028 & 0.5999899999999982 & 0.0000000000000000\end{array}$

$\begin{array}{llll}0.3999900000000025 & 0.5999899999999982 & 0.0000000000000000\end{array}$

0.20000000000000280 .79999000000000110 .0000000000000000

0.39999000000000250 .39999000000000250 .0000000000000000

$\begin{array}{llll}0.0000000000000000 & 0.7999900000000011 & 0.0000000000000000\end{array}$

$\begin{array}{llll}0.5999899999999982 & 0.5999899999999982 & 0.0000000000000000\end{array}$

$\begin{array}{llll}0.7999900000000011 & 0.5999899999999982 & 0.0000000000000000\end{array}$

$\begin{array}{lll}0.5999899999999982 & 0.7999900000000011 & 0.0000000000000000\end{array}$

$\begin{array}{llll}0.7999900000000011 & 0.3999900000000025 & 0.0000000000000000\end{array}$

0.39999000000000250 .79999000000000110 .0000000000000000

$\begin{array}{lllll}0.7999900000000011 & 0.7999900000000011 & 0.0000000000000000\end{array}$

$\begin{array}{llll}0.3333299999999966 & 0.1333300000000008 & 0.0521799999999999\end{array}$

$\begin{array}{llll}0.1333300000000008 & 0.3333299999999966 & 0.0521799999999999\end{array}$

$\begin{array}{llll}0.1333300000000008 & 0.1333300000000008 & 0.0521799999999999\end{array}$

$\begin{array}{llll}0.7333199999999991 & 0.1333300000000008 & 0.0521799999999999\end{array}$

$\begin{array}{llll}0.3333299999999966 & 0.3333299999999966 & 0.0521799999999999\end{array}$

$\begin{array}{llll}0.5333299999999994 & 0.3333299999999966 & 0.0521799999999999\end{array}$

$\begin{array}{llll}0.3333299999999966 & 0.5333299999999994 & 0.0521799999999999\end{array}$

$\begin{array}{llll}0.5333299999999994 & 0.1333300000000008 & 0.0521799999999999\end{array}$

$\begin{array}{llll}0.1333300000000008 & 0.7333199999999991 & 0.0521799999999999\end{array}$

$\begin{array}{llll}0.1333300000000008 & 0.5333299999999994 & 0.0521799999999999\end{array}$

$\begin{array}{llll}0.7333199999999991 & 0.3333299999999966 & 0.0521799999999999\end{array}$

$\begin{array}{llll}0.9333200000000019 & 0.3333299999999966 & 0.0521799999999999\end{array}$

$\begin{array}{llll}0.7333199999999991 & 0.5333299999999994 & 0.0521799999999999\end{array}$

$\begin{array}{llll}0.9333200000000019 & 0.1333300000000008 & 0.0521799999999999\end{array}$

$\begin{array}{llll}0.3333299999999966 & 0.7333199999999991 & 0.0521799999999999\end{array}$

$\begin{array}{llll}0.5333299999999994 & 0.7333199999999991 & 0.0521799999999999\end{array}$

$\begin{array}{llll}0.3333299999999966 & 0.9333200000000019 & 0.0521799999999999\end{array}$

$\begin{array}{lllll}0.5333299999999994 & 0.5333299999999994 & 0.0521799999999999\end{array}$

$\begin{array}{llll}0.1333300000000008 & 0.9333200000000019 & 0.0521799999999999\end{array}$

$\begin{array}{llll}0.7333199999999991 & 0.7333199999999991 & 0.0521799999999999\end{array}$

$\begin{array}{llll}0.9333200000000019 & 0.7333199999999991 & 0.0521799999999999\end{array}$ 
$\begin{array}{llll}0.7333199999999991 & 0.9333200000000019 & 0.0521799999999999\end{array}$

0.93332000000000190 .53332999999999940 .0521799999999999

$\begin{array}{lll}0.5333299999999994 & 0.9333200000000019 & 0.0521799999999999\end{array}$

$\begin{array}{llll}0.9333200000000019 & 0.9333200000000019 & 0.0521799999999999\end{array}$

$\begin{array}{llll}0.0662782891468112 & 0.0668301570032074 & 0.1039898023893746\end{array}$

$\begin{array}{llll}0.2668952026156570 & 0.0669137312445396 & 0.1038229868535255\end{array}$

$\begin{array}{llll}0.0666356477591821 & 0.2671306112843432 & 0.1041272406715299\end{array}$

$\begin{array}{llll}0.4677867268961302 & 0.0666115344467838 & 0.1037808195724395\end{array}$

$\begin{array}{llll}0.6671082270836881 & 0.0661076976524290 & 0.1037997663602698\end{array}$

$\begin{array}{llll}0.4662169387496912 & 0.2674408808872571 & 0.1043790385001945\end{array}$

$\begin{array}{llll}0.0670195643160536 & 0.4667922972090457 & 0.1041158080498735\end{array}$

$\begin{array}{llll}0.2686636869218317 & 0.4665477701785180 & 0.1051405237363510\end{array}$

$\begin{array}{llll}0.0672571906330760 & 0.6664283848568893 & 0.1042490116213096\end{array}$

$\begin{array}{llll}0.2669317750075147 & 0.2671062289783196 & 0.1041496925488619\end{array}$

$\begin{array}{llll}0.8663641879881940 & 0.0662806664591165 & 0.1038622683646878\end{array}$

$\begin{array}{llll}0.8665980388461245 & 0.2668274425512426 & 0.1039276441422442\end{array}$

$\begin{array}{llll}0.4674305280927620 & 0.4650811984895837 & 0.1057837701771042\end{array}$

$\begin{array}{llll}0.6655819029926843 & 0.4657186408782216 & 0.1044776231628025\end{array}$

$\begin{array}{llll}0.4652345948993260 & 0.6687088833181977 & 0.1050886560543586\end{array}$

$\begin{array}{llll}0.6663627299564424 & 0.2670873407743141 & 0.1042032884013023\end{array}$

$\begin{array}{llll}0.0670943200263530 & 0.8670017003842724 & 0.1045433844338387\end{array}$

$\begin{array}{llll}0.2672373007756287 & 0.8663669708877448 & 0.1053286718962313\end{array}$

$0.2649665957470594 \quad 0.66930161822336340 .1047953598723682$

$\begin{array}{llll}0.8669965078597517 & 0.4670009500707187 & 0.1039191032063117\end{array}$

$\begin{array}{llll}0.8669055639649266 & 0.6665221885573450 & 0.1040583112545612\end{array}$

$\begin{array}{llll}0.4668303419145813 & 0.8666930075495608 & 0.1040337492931076\end{array}$

$\begin{array}{llll}0.6675326294828670 & 0.8667103834815532 & 0.1037795840556353\end{array}$

$\begin{array}{lll}0.6671173567247355 & 0.6662559427547583 & 0.1037759678107179\end{array}$

$\begin{array}{llll}0.8668664517120359 & 0.8663921558846699 & 0.1040212650165380\end{array}$

$\begin{array}{llll}0.1969581104443197 & 0.0074769491937813 & 0.1554340996342605\end{array}$

$\begin{array}{llll}0.9992114987645623 & 0.2021767207999880 & 0.1565017847848301\end{array}$

$\begin{array}{llll}0.9957269810948732 & 0.0046775675596586 & 0.1561218564903190\end{array}$

$\begin{array}{lll}0.6013508494860774 & 0.9983357757804009 & 0.1563887090431239\end{array}$

$\begin{array}{llll}0.1991235799939263 & 0.2016972634506938 & 0.1565027754180957\end{array}$

$\begin{array}{llll}0.4002611437691300 & 0.1963411503865202 & 0.1561461366826258\end{array}$

$\begin{array}{llll}0.1978000507537312 & 0.3996731865433760 & 0.1562999639876296\end{array}$

$\begin{array}{llll}0.4003580813424706 & 0.0035406564921295 & 0.1553236122300639\end{array}$

0.99927632337436630 .60028286711362950 .1564013199044798

0.99998368334198940 .40083641147599060 .1565428218768667

$\begin{array}{lllll}0.6030559284095254 & 0.1941934779978766 & 0.1552028744806067\end{array}$

$\begin{array}{llll}0.8028627531117898 & 0.1975941224064294 & 0.1557695128899030\end{array}$

$\begin{array}{llll}0.6085795353676868 & 0.3928145185135325 & 0.1580807722550157\end{array}$

$\begin{array}{llll}0.8000996520827945 & 0.9991189817277214 & 0.1564200567720191\end{array}$

$\begin{array}{llll}0.1970137588560960 & 0.6016878346656440 & 0.1559919045474759\end{array}$

$\begin{array}{llll}0.3976610655310389 & 0.6015635063863154 & 0.1548628666796744\end{array}$

$\begin{array}{llll}0.1915337084705195 & 0.8115013354786873 & 0.1577308135325643\end{array}$

$\begin{array}{llll}0.4014845144316339 & 0.3902622898897180 & 0.1581295435885425\end{array}$ 
0.99701029449292840 .80116479254430040 .1561089468720494

$\begin{array}{lll}0.6028134109266904 & 0.5999719137961119 & 0.1551169646117576\end{array}$

$\begin{array}{llll}0.8007417416756278 & 0.5999074307146141 & 0.1565153892579229\end{array}$

0.60471983103144940 .79959630580887350 .1551913724510138

$\begin{array}{llll}0.8036992484746117 & 0.3999262801872312 & 0.1556917686385061\end{array}$

$\begin{array}{lllll}0.4025186888833711 & 0.8080082526203488 & 0.1576928110650936\end{array}$

$\begin{array}{llll}0.8008224351956831 & 0.7995523681196807 & 0.1565462944521105\end{array}$

$\begin{array}{llll}0.4023830401517971 & 0.3935157810736132 & 0.2088228761662190\end{array}$

$\begin{array}{llll}0.1952416650992546 & 0.8265727085571446 & 0.2084858944866702\end{array}$

$\begin{array}{llll}0.6155765195313180 & 0.3836686409781305 & 0.2133203189514480\end{array}$

$\begin{array}{llll}0.4053579019406834 & 0.8241218135718446 & 0.2130098162500809\end{array}$

$\begin{array}{llll}0.5106802016908256 & 0.4963698814727781 & 0.2189574303884314\end{array}$

$0.2910342393086304 \quad 0.61036760469783190 .2134390853708695$

$\begin{array}{llll}0.3991304435235446 & 0.4967932992147991 & 0.2144951298953424\end{array}$

$\begin{array}{llll}0.4060138634649473 & 0.7151349316477986 & 0.2191685336582460\end{array}$

$\begin{array}{llll}0.5133880212212839 & 0.6041008836179104 & 0.2218177273139369\end{array}$

$\begin{array}{llll}0.2919697716978761 & 0.7210113712154052 & 0.2145946153612956\end{array}$

$\begin{array}{llll}0.6000670946226200 & 0.6016411032528232 & 0.2238671901970942\end{array}$

$\begin{array}{llll}0.2037451440746895 & 0.6131954226439920 & 0.2132161093707593\end{array}$

$\begin{array}{llll}0.3252603798269265 & 0.8988527428399314 & 0.2194802022263139\end{array}$

$\begin{array}{llll}0.4792375032865105 & 0.8282256372243081 & 0.2208419721337491\end{array}$

$\begin{array}{llll}0.6947881773821180 & 0.3740361375695321 & 0.2221971848423553\end{array}$

$\begin{array}{llll}0.6028349648148439 & 0.3130894080117083 & 0.2196535531861313\end{array}$

Zwitterion on Cu - VASP - optB88-vdW - Start B30

$\mathrm{Cu} \mathrm{O} N \mathrm{C} \mathrm{H}$

1.00000000000000

$\begin{array}{lll}12.7810000000000006 & 0.0000000000000000 & 0.0000000000000000\end{array}$

$\begin{array}{llll}6.3905000000000003 & 11.0686699999999991 & 0.0000000000000000\end{array}$

$\begin{array}{llll}0.0000000000000000 & 0.0000000000000000 & 40.0000000000000000\end{array}$

$\begin{array}{lllll}100 & 2 & 2 & 6 & 6\end{array}$

Direct

$\begin{array}{llll}0.2000000000000028 & 0.0000000000000000 & 0.0000000000000000\end{array}$

$\begin{array}{lllll}0.0000000000000000 & 0.2000000000000028 & 0.0000000000000000\end{array}$

$\begin{array}{lllll}0.0000000000000000 & 0.0000000000000000 & 0.0000000000000000\end{array}$

$\begin{array}{lllll}0.5999899999999982 & 0.0000000000000000 & 0.0000000000000000\end{array}$

$\begin{array}{lllll}0.2000000000000028 & 0.2000000000000028 & 0.0000000000000000\end{array}$

$\begin{array}{lllll}0.3999900000000025 & 0.2000000000000028 & 0.0000000000000000\end{array}$

$\begin{array}{lllll}0.2000000000000028 & 0.3999900000000025 & 0.0000000000000000\end{array}$

$\begin{array}{llll}0.3999900000000025 & 0.0000000000000000 & 0.0000000000000000\end{array}$

$\begin{array}{lllll}0.0000000000000000 & 0.5999899999999982 & 0.0000000000000000\end{array}$

$\begin{array}{lllll}0.0000000000000000 & 0.3999900000000025 & 0.0000000000000000\end{array}$

$\begin{array}{llllll}0.5999899999999982 & 0.2000000000000028 & 0.0000000000000000\end{array}$

$\begin{array}{llllll}0.7999900000000011 & 0.2000000000000028 & 0.0000000000000000\end{array}$

$\begin{array}{lllll}0.5999899999999982 & 0.3999900000000025 & 0.0000000000000000\end{array}$

$\begin{array}{llll}0.7999900000000011 & 0.0000000000000000 & 0.0000000000000000\end{array}$ 
0.20000000000000280 .59998999999999820 .0000000000000000

$\begin{array}{lll}0.3999900000000025 & 0.5999899999999982 & 0.0000000000000000\end{array}$

0.20000000000000280 .79999000000000110 .0000000000000000

0.39999000000000250 .39999000000000250 .0000000000000000

$\begin{array}{llll}0.0000000000000000 & 0.7999900000000011 & 0.0000000000000000\end{array}$

$\begin{array}{lll}0.5999899999999982 & 0.5999899999999982 & 0.0000000000000000\end{array}$

$\begin{array}{llll}0.7999900000000011 & 0.5999899999999982 & 0.0000000000000000\end{array}$

$\begin{array}{lllll}0.5999899999999982 & 0.7999900000000011 & 0.0000000000000000\end{array}$

$\begin{array}{lll}0.7999900000000011 & 0.3999900000000025 & 0.0000000000000000\end{array}$

0.39999000000000250 .79999000000000110 .0000000000000000

$\begin{array}{lllll}0.7999900000000011 & 0.7999900000000011 & 0.0000000000000000\end{array}$

$\begin{array}{llll}0.3333299999999966 & 0.1333300000000008 & 0.0521799999999999\end{array}$

$\begin{array}{llll}0.1333300000000008 & 0.3333299999999966 & 0.0521799999999999\end{array}$

$\begin{array}{llll}0.1333300000000008 & 0.1333300000000008 & 0.0521799999999999\end{array}$

$\begin{array}{llll}0.7333199999999991 & 0.1333300000000008 & 0.0521799999999999\end{array}$

$\begin{array}{llll}0.3333299999999966 & 0.3333299999999966 & 0.0521799999999999\end{array}$

$\begin{array}{lllll}0.5333299999999994 & 0.3333299999999966 & 0.0521799999999999\end{array}$

$\begin{array}{lll}0.3333299999999966 & 0.5333299999999994 & 0.0521799999999999\end{array}$

$\begin{array}{llll}0.5333299999999994 & 0.1333300000000008 & 0.0521799999999999\end{array}$

$\begin{array}{llll}0.1333300000000008 & 0.7333199999999991 & 0.0521799999999999\end{array}$

$\begin{array}{llll}0.1333300000000008 & 0.5333299999999994 & 0.0521799999999999\end{array}$

$\begin{array}{llll}0.7333199999999991 & 0.3333299999999966 & 0.0521799999999999\end{array}$

$\begin{array}{llll}0.9333200000000019 & 0.3333299999999966 & 0.0521799999999999\end{array}$

$\begin{array}{llll}0.7333199999999991 & 0.5333299999999994 & 0.0521799999999999\end{array}$

$\begin{array}{llll}0.9333200000000019 & 0.1333300000000008 & 0.0521799999999999\end{array}$

$\begin{array}{llll}0.3333299999999966 & 0.7333199999999991 & 0.0521799999999999\end{array}$

$\begin{array}{llll}0.5333299999999994 & 0.7333199999999991 & 0.0521799999999999\end{array}$

$\begin{array}{llll}0.3333299999999966 & 0.9333200000000019 & 0.0521799999999999\end{array}$

0.53332999999999940 .53332999999999940 .0521799999999999

$\begin{array}{llll}0.1333300000000008 & 0.9333200000000019 & 0.0521799999999999\end{array}$

$\begin{array}{llll}0.7333199999999991 & 0.7333199999999991 & 0.0521799999999999\end{array}$

$\begin{array}{llll}0.9333200000000019 & 0.7333199999999991 & 0.0521799999999999\end{array}$

$\begin{array}{llll}0.7333199999999991 & 0.9333200000000019 & 0.0521799999999999\end{array}$

$\begin{array}{lll}0.9333200000000019 & 0.5333299999999994 & 0.0521799999999999\end{array}$

0.53332999999999940 .93332000000000190 .0521799999999999

0.93332000000000190 .93332000000000190 .0521799999999999

$\begin{array}{llll}0.0659916660060718 & 0.0662731216292357 & 0.1042085600286404\end{array}$

$\begin{array}{llll}0.2662888275221351 & 0.0658494124776673 & 0.1040543606844870\end{array}$

$\begin{array}{llll}0.0658147706553299 & 0.2661125338089287 & 0.1041553363992116\end{array}$

$\begin{array}{llll}0.4659972030848253 & 0.0670493819029024 & 0.1045560294156923\end{array}$

$\begin{array}{llll}0.6670303857752063 & 0.0658073273889565 & 0.1046399397806403\end{array}$

$\begin{array}{llll}0.4682564490307211 & 0.2630387182492954 & 0.1042206203423144\end{array}$

$\begin{array}{llll}0.0679610092688183 & 0.4645582778051688 & 0.1053551692856504\end{array}$

$\begin{array}{llll}0.2643099732834711 & 0.4654592903384621 & 0.1034106065013607\end{array}$

$\begin{array}{llll}0.0655241225885790 & 0.6661760878597106 & 0.1041080857918390\end{array}$

$0.2661039640089448 \quad 0.26661178019850330 .1050294894235665$

$\begin{array}{llll}0.8673158556530499 & 0.0651259416698833 & 0.1040378847495312\end{array}$ 
0.86599970851851990 .26639536533566820 .1039914832632995

$\begin{array}{llll}0.4682281725599918 & 0.4654562961557242 & 0.1027007560961342\end{array}$

$\begin{array}{llll}0.6660006985916365 & 0.4680771148039298 & 0.1051117061995544\end{array}$

$0.4682828673452294 \quad 0.6671894059613706 \quad 0.1039952807383834$

$\begin{array}{llll}0.6663120297859387 & 0.2644975980211309 & 0.1049432248439180\end{array}$

$\begin{array}{llll}0.0685644443628286 & 0.8649727420159325 & 0.1055617889287009\end{array}$

$\begin{array}{llll}0.2651597082253356 & 0.8654410706783083 & 0.1049010190908406\end{array}$

$\begin{array}{llll}0.2641884689372579 & 0.6679808281967267 & 0.1037610005982415\end{array}$

$\begin{array}{llll}0.8665617217004077 & 0.4664822294598598 & 0.1040504598220877\end{array}$

$\begin{array}{llll}0.8661592014981782 & 0.6658651594132936 & 0.1039951068236609\end{array}$

$\begin{array}{llll}0.4669048105421076 & 0.8669236485167726 & 0.1038660805343203\end{array}$

$\begin{array}{llll}0.6672234487576483 & 0.8657348563032307 & 0.1038429296517616\end{array}$

$\begin{array}{llll}0.6661483597965601 & 0.6658623145089478 & 0.1050417566188271\end{array}$

$\begin{array}{llll}0.8667891245080012 & 0.8657314118318993 & 0.1039847454915129\end{array}$

$\begin{array}{llll}0.1979917402045716 & 0.0007597921712102 & 0.1563997148572258\end{array}$

$\begin{array}{llll}0.9986725630981381 & 0.1989986554253456 & 0.1566135040273679\end{array}$

$\begin{array}{llll}0.0002185414389094 & 0.9986600392266822 & 0.1567307613687565\end{array}$

$\begin{array}{llll}0.6003677183706141 & 0.9959939391972105 & 0.1561157864895862\end{array}$

$\begin{array}{llll}0.1966220972050559 & 0.1969517164770436 & 0.1561383271241887\end{array}$

$\begin{array}{llll}0.3975254497715967 & 0.1966784843062065 & 0.1569461376620350\end{array}$

$\begin{array}{llll}0.1958229842104563 & 0.3965637245618060 & 0.1593393113882611\end{array}$

$\begin{array}{llll}0.3988895202767035 & 0.9997892242059947 & 0.1566007158926492\end{array}$

$\begin{array}{llll}0.9975374798632132 & 0.5993262582740327 & 0.1563975881046536\end{array}$

$\begin{array}{llll}0.9979031100262946 & 0.3986063832818776 & 0.1566441535749210\end{array}$

$\begin{array}{llll}0.6033916990950559 & 0.1913773576248980 & 0.1580701791091766\end{array}$

$\begin{array}{llll}0.8029528671925991 & 0.1967876412806307 & 0.1560666672785322\end{array}$

$\begin{array}{llll}0.6039374160272336 & 0.3965322431662590 & 0.1553460063631486\end{array}$

$\begin{array}{llll}0.8027963384651083 & 0.9961740303063741 & 0.1563095068091055\end{array}$

$\begin{array}{llll}0.1965946040890642 & 0.5980960206960548 & 0.1546548515121281\end{array}$

$\begin{array}{llll}0.4007959956322675 & 0.5990470013075081 & 0.1518403075791680\end{array}$

$\begin{array}{llll}0.2002232853912237 & 0.7993791168973748 & 0.1608434780917166\end{array}$

$\begin{array}{llll}0.4002483702337982 & 0.4006719679960578 & 0.1514523271762647\end{array}$

$\begin{array}{llll}0.9991612176853274 & 0.7985551747843483 & 0.1564527966905585\end{array}$

$\begin{array}{llll}0.6000645343315235 & 0.6034081581336020 & 0.1594411893125914\end{array}$

0.80041657363716880 .59905994171077650 .1565524857731404

$\begin{array}{llll}0.6001755129902705 & 0.8011916766186852 & 0.1563387507037782\end{array}$

$\begin{array}{lllll}0.8012973241511936 & 0.3984920183786674 & 0.1562994287022845\end{array}$

$\begin{array}{llll}0.3990875228558881 & 0.8046854724205302 & 0.1549428466144647\end{array}$

$\begin{array}{llll}0.7999570154951828 & 0.7987146983444091 & 0.1564671789060164\end{array}$

$\begin{array}{llll}0.3174732056039755 & 0.3356793040190458 & 0.2020867469665192\end{array}$

$\begin{array}{llll}0.2963196392595352 & 0.7253615360092845 & 0.2059182436028660\end{array}$

$\begin{array}{llll}0.5648889155287296 & 0.2191959302940293 & 0.2116810021073356\end{array}$

$\begin{array}{llll}0.5443156690851617 & 0.6139455865082885 & 0.2128773473614826\end{array}$

$\begin{array}{llll}0.4957209812785989 & 0.3475317326674500 & 0.2131664557081707\end{array}$

$\begin{array}{llll}0.3012484033637226 & 0.5330120163016488 & 0.2068910900738604\end{array}$

$\begin{array}{llll}0.3688023085761296 & 0.4034378049394998 & 0.2074096773524048\end{array}$

$\begin{array}{llll}0.4857054628572884 & 0.5440369605353326 & 0.2137072182633107\end{array}$ 
0.55237188724336730 .41738042625349030 .2162365659593433

$\begin{array}{llll}0.3574503556505078 & 0.6060641396271718 & 0.2089512979837121\end{array}$

$0.6493757490239788 \quad 0.37247305213446140 .2215129002740406$

$\begin{array}{llll}0.2026039291658631 & 0.5776600184039663 & 0.2100271574327665\end{array}$

$\begin{array}{llll}0.4844992398768628 & 0.7024755212508731 & 0.2183598195436090\end{array}$

$\begin{array}{llll}0.6202587860057319 & 0.5809137851128440 & 0.2272869711171296\end{array}$

$\begin{array}{llll}0.6448429819203297 & 0.1817517303557659 & 0.2242535912714754\end{array}$

$\begin{array}{llll}0.5148680686065938 & 0.1800611018828141 & 0.2183286822818429\end{array}$

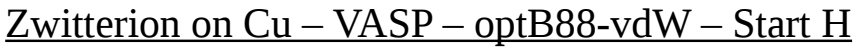

$\mathrm{Cu} \mathrm{O} \mathrm{N} \mathrm{C} \mathrm{H}$

1.00000000000000

$\begin{array}{lll}12.7810000000000006 & 0.0000000000000000 & 0.0000000000000000\end{array}$

$\begin{array}{lll}6.3905000000000003 & 11.0686699999999991 & 0.0000000000000000\end{array}$

$0.0000000000000000 \quad 0.0000000000000000 \quad 40.0000000000000000$

Direct

0.20000000000000280 .00000000000000000 .0000000000000000

$\begin{array}{lll}0.0000000000000000 & 0.2000000000000028 & 0.0000000000000000\end{array}$

$\begin{array}{lll}0.0000000000000000 & 0.0000000000000000 & 0.0000000000000000\end{array}$

$\begin{array}{lll}0.5999899999999982 & 0.0000000000000000 & 0.0000000000000000\end{array}$

$\begin{array}{lll}0.2000000000000028 & 0.2000000000000028 & 0.0000000000000000\end{array}$

$\begin{array}{lll}0.3999900000000025 & 0.2000000000000028 & 0.0000000000000000\end{array}$

$\begin{array}{llll}0.2000000000000028 & 0.3999900000000025 & 0.0000000000000000\end{array}$

$\begin{array}{llll}0.3999900000000025 & 0.0000000000000000 & 0.0000000000000000\end{array}$

$\begin{array}{llll}0.0000000000000000 & 0.5999899999999982 & 0.0000000000000000\end{array}$

$\begin{array}{lll}0.0000000000000000 & 0.3999900000000025 & 0.0000000000000000\end{array}$

$\begin{array}{llll}0.5999899999999982 & 0.2000000000000028 & 0.0000000000000000\end{array}$

$\begin{array}{llll}0.7999900000000011 & 0.2000000000000028 & 0.0000000000000000\end{array}$

$\begin{array}{llll}0.5999899999999982 & 0.3999900000000025 & 0.0000000000000000\end{array}$

$\begin{array}{llll}0.7999900000000011 & 0.0000000000000000 & 0.0000000000000000\end{array}$

$\begin{array}{lll}0.2000000000000028 & 0.5999899999999982 & 0.0000000000000000\end{array}$

0.39999000000000250 .59998999999999820 .0000000000000000

$\begin{array}{llll}0.2000000000000028 & 0.7999900000000011 & 0.0000000000000000\end{array}$

$\begin{array}{lll}0.3999900000000025 & 0.3999900000000025 & 0.0000000000000000\end{array}$

$\begin{array}{llll}0.0000000000000000 & 0.7999900000000011 & 0.0000000000000000\end{array}$

$\begin{array}{llll}0.5999899999999982 & 0.5999899999999982 & 0.0000000000000000\end{array}$

$\begin{array}{llll}0.7999900000000011 & 0.5999899999999982 & 0.0000000000000000\end{array}$

$\begin{array}{lllll}0.5999899999999982 & 0.7999900000000011 & 0.0000000000000000\end{array}$

$\begin{array}{llll}0.7999900000000011 & 0.3999900000000025 & 0.0000000000000000\end{array}$

0.39999000000000250 .79999000000000110 .0000000000000000

$\begin{array}{llll}0.7999900000000011 & 0.7999900000000011 & 0.0000000000000000\end{array}$

$\begin{array}{llll}0.3333299999999966 & 0.1333300000000008 & 0.0521799999999999\end{array}$

$\begin{array}{llll}0.1333300000000008 & 0.3333299999999966 & 0.0521799999999999\end{array}$

$\begin{array}{llll}0.1333300000000008 & 0.1333300000000008 & 0.0521799999999999\end{array}$

$\begin{array}{llll}0.7333199999999991 & 0.1333300000000008 & 0.0521799999999999\end{array}$ 
$\begin{array}{llll}0.3333299999999966 & 0.3333299999999966 & 0.0521799999999999\end{array}$

$\begin{array}{lllll}0.5333299999999994 & 0.3333299999999966 & 0.0521799999999999\end{array}$

$\begin{array}{lllll}0.3333299999999966 & 0.5333299999999994 & 0.0521799999999999\end{array}$

$\begin{array}{lll}0.5333299999999994 & 0.1333300000000008 & 0.0521799999999999\end{array}$

$\begin{array}{llll}0.1333300000000008 & 0.7333199999999991 & 0.0521799999999999\end{array}$

$\begin{array}{llll}0.1333300000000008 & 0.5333299999999994 & 0.0521799999999999\end{array}$

$\begin{array}{lllll}0.7333199999999991 & 0.3333299999999966 & 0.0521799999999999\end{array}$

$\begin{array}{llll}0.9333200000000019 & 0.3333299999999966 & 0.0521799999999999\end{array}$

$\begin{array}{lll}0.7333199999999991 & 0.5333299999999994 & 0.0521799999999999\end{array}$

$\begin{array}{llll}0.9333200000000019 & 0.1333300000000008 & 0.0521799999999999\end{array}$

$\begin{array}{llll}0.3333299999999966 & 0.7333199999999991 & 0.0521799999999999\end{array}$

$\begin{array}{lllll}0.5333299999999994 & 0.7333199999999991 & 0.0521799999999999\end{array}$

$\begin{array}{llll}0.3333299999999966 & 0.9333200000000019 & 0.0521799999999999\end{array}$

$\begin{array}{lll}0.5333299999999994 & 0.5333299999999994 & 0.0521799999999999\end{array}$

$\begin{array}{llll}0.1333300000000008 & 0.9333200000000019 & 0.0521799999999999\end{array}$

$\begin{array}{llll}0.7333199999999991 & 0.7333199999999991 & 0.0521799999999999\end{array}$

$\begin{array}{llll}0.9333200000000019 & 0.7333199999999991 & 0.0521799999999999\end{array}$

$\begin{array}{llll}0.7333199999999991 & 0.9333200000000019 & 0.0521799999999999\end{array}$

$\begin{array}{lll}0.9333200000000019 & 0.5333299999999994 & 0.0521799999999999\end{array}$

$\begin{array}{lll}0.5333299999999994 & 0.9333200000000019 & 0.0521799999999999\end{array}$

$\begin{array}{llll}0.9333200000000019 & 0.9333200000000019 & 0.0521799999999999\end{array}$

$\begin{array}{llll}0.0663815485868677 & 0.0668221678574907 & 0.1040777969334163\end{array}$

$0.2668976752667786 \quad 0.06684422438569290 .1039122384419848$

$\begin{array}{llll}0.0666539248029717 & 0.2671458336747335 & 0.1041842190358227\end{array}$

$\begin{array}{llll}0.4677131255968640 & 0.0666651674416290 & 0.1038563600347473\end{array}$

$\begin{array}{llll}0.6670558833866276 & 0.0662755826594241 & 0.1038639744149642\end{array}$

$\begin{array}{llll}0.4661949006659069 & 0.2675718315707319 & 0.1045522398919111\end{array}$

$\begin{array}{llll}0.0670565576901253 & 0.4668713030788277 & 0.1041785213935397\end{array}$

$\begin{array}{llll}0.2687275067258865 & 0.4666194119946996 & 0.1052207354558552\end{array}$

$\begin{array}{llll}0.0673400380805555 & 0.6665006329525262 & 0.1043288234423121\end{array}$

$\begin{array}{llll}0.2669474356035593 & 0.2671688292884156 & 0.1042206470802666\end{array}$

$\begin{array}{lllll}0.8664166628525996 & 0.0663850216566197 & 0.1039342103852466\end{array}$

$\begin{array}{llll}0.8665532872781583 & 0.2669460322607530 & 0.1040049998672553\end{array}$

$\begin{array}{llll}0.4674229626011323 & 0.4651854632629571 & 0.1059337166884657\end{array}$

$\begin{array}{llll}0.6655366936721356 & 0.4658431970291475 & 0.1046766221997902\end{array}$

$\begin{array}{llll}0.4652970929832785 & 0.6687372653284260 & 0.1051727940267645\end{array}$

$\begin{array}{llll}0.6663452627511394 & 0.2672137353683461 & 0.1043344060730482\end{array}$

$\begin{array}{llll}0.0672770191227587 & 0.8669758647692010 & 0.1048229572732348\end{array}$

0.26720332020653430 .86638381600652660 .1054914238290395

$\begin{array}{llll}0.2650900398490034 & 0.6692775753110497 & 0.1049367069238375\end{array}$

$\begin{array}{llll}0.8669176372320212 & 0.4670624918564645 & 0.1039812212078084\end{array}$

$\begin{array}{llll}0.8669269375043425 & 0.6665933883992816 & 0.1041204239334303\end{array}$

$\begin{array}{llll}0.4667199056786362 & 0.8667067559986092 & 0.1041834195068073\end{array}$

$\begin{array}{llll}0.6674927126319163 & 0.8667820007910009 & 0.1038341405424255\end{array}$

$\begin{array}{lllll}0.6670558178614191 & 0.6663431181007118 & 0.1038494967411378\end{array}$

$\begin{array}{llll}0.8669292794789889 & 0.8664805979902241 & 0.1040776272255527\end{array}$

$\begin{array}{llll}0.1969908778811676 & 0.0074641543776447 & 0.1556042426622728\end{array}$ 
$\begin{array}{llll}0.9992003619607059 & 0.2023215860285600 & 0.1565417228769022\end{array}$

0.99578909352244750 .00480038790771690 .1562165991889922

$\begin{array}{llll}0.6013212702993328 & 0.9984916584623261 & 0.1564130082456696\end{array}$

$\begin{array}{llll}0.1991119950457181 & 0.2017989712424250 & 0.1565403209311707\end{array}$

$\begin{array}{llll}0.4002407217558213 & 0.1965580185909401 & 0.1562005082670220\end{array}$

$\begin{array}{llll}0.1978538441324401 & 0.3997586637755116 & 0.1563361954642172\end{array}$

$\begin{array}{llll}0.4003393440360209 & 0.0035157790785257 & 0.1555274155614152\end{array}$

$\begin{array}{llll}0.9992638803530065 & 0.6003682131022579 & 0.1564383517027269\end{array}$

$\begin{array}{llll}0.9999616974670220 & 0.4009742699224457 & 0.1565726200094222\end{array}$

$\begin{array}{llll}0.6030206705808512 & 0.1944416470556656 & 0.1553623406323375\end{array}$

0.80273434760297020 .19785127064105850 .1558629263915937

$\begin{array}{llll}0.6085183137888471 & 0.3930456456174861 & 0.1583209174199337\end{array}$

$\begin{array}{llll}0.8000997644347018 & 0.9992468832827072 & 0.1564463928585577\end{array}$

$\begin{array}{llll}0.1971533929033173 & 0.6017232619944012 & 0.1560705068933268\end{array}$

$\begin{array}{llll}0.3979159636329451 & 0.6016998168320291 & 0.1550262513277470\end{array}$

$\begin{array}{llll}0.1916605813842612 & 0.8115008776047768 & 0.1580718902174103\end{array}$

$\begin{array}{llll}0.4014514989677721 & 0.3905496393622659 & 0.1583697690461652\end{array}$

$\begin{array}{lll}0.9970362209961672 & 0.8011929197669735 & 0.1562013122394100\end{array}$

$\begin{array}{llll}0.6027235699577768 & 0.6001971535386057 & 0.1552896979781686\end{array}$

$\begin{array}{llll}0.8007579174350371 & 0.6000519677975328 & 0.1565593590323245\end{array}$

$\begin{array}{llll}0.6045593233125930 & 0.7997774701322723 & 0.1553465517271869\end{array}$

$\begin{array}{llll}0.8036049077761309 & 0.4000718169824951 & 0.1558072530535865\end{array}$

$\begin{array}{llll}0.4024954184046767 & 0.8080609975492083 & 0.1579754469591890\end{array}$

$\begin{array}{llll}0.8007972019393108 & 0.7996720261669377 & 0.1565771698993036\end{array}$

$\begin{array}{llll}0.4026747265790844 & 0.3930147723061543 & 0.2091756894714503\end{array}$

$\begin{array}{llll}0.1950084238279379 & 0.8259473477836758 & 0.2089446171005205\end{array}$

$\begin{array}{llll}0.6157253650402494 & 0.3834436128741585 & 0.2138110994477558\end{array}$

$\begin{array}{llll}0.4048594605702290 & 0.8239692440170933 & 0.2135413313639111\end{array}$

$\begin{array}{llll}0.5107232547224428 & 0.4960553095201646 & 0.2194214753245058\end{array}$

$\begin{array}{llll}0.2910581139830313 & 0.6097791128060156 & 0.2139345839833356\end{array}$

$\begin{array}{llll}0.3992350412067600 & 0.4963268142419949 & 0.2149400861072305\end{array}$

$\begin{array}{llll}0.4057954168207659 & 0.7148091977273615 & 0.2196234661032357\end{array}$

$\begin{array}{llll}0.5132541292762083 & 0.6038797053800388 & 0.2222476352759410\end{array}$

$\begin{array}{llll}0.2918201392372877 & 0.7204745456856403 & 0.2150593804022510\end{array}$

$\begin{array}{llll}0.5998908600314481 & 0.6015447586266007 & 0.2242971324106284\end{array}$

$\begin{array}{llll}0.2038540380368959 & 0.6124462605519972 & 0.2136850478515935\end{array}$

$\begin{array}{llll}0.3245103996602568 & 0.8984675125276231 & 0.2199818405631004\end{array}$

$\begin{array}{llll}0.4784043564514025 & 0.8283895871986324 & 0.2215310477045440\end{array}$

$\begin{array}{llll}0.6948143624023159 & 0.3738979382218889 & 0.2227680034554725\end{array}$

$\begin{array}{llll}0.6030626515031058 & 0.3127908769079790 & 0.2201188102420706\end{array}$

Zwitterion on $\mathrm{Cu}$ - VASP - optB88-vdW - Start H30

$\mathrm{Cu} \mathrm{O} \mathrm{N} \mathrm{C} \mathrm{H}$

1.00000000000000

$\begin{array}{lll}12.7810000000000006 & 0.0000000000000000 & 0.0000000000000000\end{array}$

$\begin{array}{llll}6.3905000000000003 & 11.0686699999999991 & 0.0000000000000000\end{array}$ 


$\begin{array}{cccccc}0.0000000000000000 & 0.0000000000000000 & 40.0000000000000000 \\ 100 & 2 & 2 & 6 & 6 & \end{array}$

Direct

$\begin{array}{llll}0.2000000000000028 & 0.0000000000000000 & 0.0000000000000000\end{array}$

$\begin{array}{lllll}0.0000000000000000 & 0.2000000000000028 & 0.0000000000000000\end{array}$

$\begin{array}{lllll}0.0000000000000000 & 0.0000000000000000 & 0.0000000000000000\end{array}$

$\begin{array}{lllll}0.5999899999999982 & 0.0000000000000000 & 0.0000000000000000\end{array}$

$\begin{array}{lllll}0.2000000000000028 & 0.2000000000000028 & 0.0000000000000000\end{array}$

$\begin{array}{lllll}0.3999900000000025 & 0.2000000000000028 & 0.0000000000000000\end{array}$

$\begin{array}{lllll}0.2000000000000028 & 0.3999900000000025 & 0.0000000000000000\end{array}$

$\begin{array}{llll}0.3999900000000025 & 0.0000000000000000 & 0.0000000000000000\end{array}$

$\begin{array}{lllll}0.0000000000000000 & 0.5999899999999982 & 0.0000000000000000\end{array}$

$\begin{array}{lllll}0.0000000000000000 & 0.3999900000000025 & 0.0000000000000000\end{array}$

$\begin{array}{lllll}0.5999899999999982 & 0.2000000000000028 & 0.0000000000000000\end{array}$

$\begin{array}{lllll}0.7999900000000011 & 0.2000000000000028 & 0.0000000000000000\end{array}$

$\begin{array}{lllll}0.5999899999999982 & 0.3999900000000025 & 0.0000000000000000\end{array}$

$\begin{array}{llll}0.7999900000000011 & 0.0000000000000000 & 0.0000000000000000\end{array}$

$\begin{array}{lllll}0.2000000000000028 & 0.5999899999999982 & 0.0000000000000000\end{array}$

$\begin{array}{lllll}0.3999900000000025 & 0.5999899999999982 & 0.0000000000000000\end{array}$

$\begin{array}{lllll}0.2000000000000028 & 0.7999900000000011 & 0.0000000000000000\end{array}$

$\begin{array}{llll}0.3999900000000025 & 0.3999900000000025 & 0.0000000000000000\end{array}$

$\begin{array}{lllll}0.0000000000000000 & 0.7999900000000011 & 0.0000000000000000\end{array}$

$\begin{array}{lllll}0.5999899999999982 & 0.5999899999999982 & 0.0000000000000000\end{array}$

$\begin{array}{llllll}0.7999900000000011 & 0.5999899999999982 & 0.0000000000000000\end{array}$

$\begin{array}{lllll}0.5999899999999982 & 0.7999900000000011 & 0.0000000000000000\end{array}$

$\begin{array}{lllll}0.7999900000000011 & 0.3999900000000025 & 0.0000000000000000\end{array}$

$\begin{array}{lllll}0.3999900000000025 & 0.7999900000000011 & 0.0000000000000000\end{array}$

$\begin{array}{lllll}0.7999900000000011 & 0.7999900000000011 & 0.0000000000000000\end{array}$

$\begin{array}{lllll}0.3333299999999966 & 0.1333300000000008 & 0.0521799999999999\end{array}$

$\begin{array}{lllll}0.1333300000000008 & 0.3333299999999966 & 0.0521799999999999\end{array}$

$\begin{array}{lllll}0.1333300000000008 & 0.1333300000000008 & 0.0521799999999999\end{array}$

$\begin{array}{llllll}0.7333199999999991 & 0.1333300000000008 & 0.0521799999999999\end{array}$

$\begin{array}{lllll}0.3333299999999966 & 0.3333299999999966 & 0.0521799999999999\end{array}$

$\begin{array}{lllll}0.5333299999999994 & 0.3333299999999966 & 0.0521799999999999\end{array}$

$\begin{array}{lllll}0.3333299999999966 & 0.5333299999999994 & 0.0521799999999999\end{array}$

$\begin{array}{lllll}0.5333299999999994 & 0.1333300000000008 & 0.0521799999999999\end{array}$

$\begin{array}{lllll}0.1333300000000008 & 0.7333199999999991 & 0.0521799999999999\end{array}$

$\begin{array}{lllll}0.1333300000000008 & 0.5333299999999994 & 0.0521799999999999\end{array}$

$\begin{array}{lllll}0.7333199999999991 & 0.3333299999999966 & 0.0521799999999999\end{array}$

$\begin{array}{lllll}0.9333200000000019 & 0.3333299999999966 & 0.0521799999999999\end{array}$

$\begin{array}{llllll}0.7333199999999991 & 0.5333299999999994 & 0.0521799999999999\end{array}$

$\begin{array}{lllll}0.9333200000000019 & 0.1333300000000008 & 0.0521799999999999\end{array}$

$\begin{array}{lllll}0.3333299999999966 & 0.7333199999999991 & 0.0521799999999999\end{array}$

$\begin{array}{lllll}0.5333299999999994 & 0.7333199999999991 & 0.0521799999999999\end{array}$

$\begin{array}{lllll}0.3333299999999966 & 0.9333200000000019 & 0.0521799999999999\end{array}$

$\begin{array}{lllll}0.5333299999999994 & 0.5333299999999994 & 0.0521799999999999\end{array}$

$\begin{array}{lllll}0.1333300000000008 & 0.9333200000000019 & 0.0521799999999999\end{array}$ 
$\begin{array}{llll}0.7333199999999991 & 0.7333199999999991 & 0.0521799999999999\end{array}$

$\begin{array}{llll}0.9333200000000019 & 0.7333199999999991 & 0.0521799999999999\end{array}$

$\begin{array}{llll}0.7333199999999991 & 0.9333200000000019 & 0.0521799999999999\end{array}$

$\begin{array}{lll}0.9333200000000019 & 0.5333299999999994 & 0.0521799999999999\end{array}$

0.53332999999999940 .93332000000000190 .0521799999999999

$\begin{array}{llll}0.9333200000000019 & 0.9333200000000019 & 0.0521799999999999\end{array}$

$\begin{array}{llll}0.0671243328245601 & 0.0666202273047539 & 0.1042115605000654\end{array}$

$\begin{array}{llll}0.2672622943346981 & 0.0666045966440331 & 0.1041126502743216\end{array}$

$\begin{array}{llll}0.0673082552288107 & 0.2665443587920975 & 0.1042099113144280\end{array}$

$\begin{array}{llll}0.4668920704871877 & 0.0677276921023040 & 0.1048448485831268\end{array}$

$\begin{array}{llll}0.6677458033997715 & 0.0665305579001287 & 0.1048254988347538\end{array}$

$\begin{array}{llll}0.4694468759167205 & 0.2637714086879918 & 0.1044938525804706\end{array}$

$\begin{array}{llll}0.0694140352696545 & 0.4651205628393822 & 0.1054099828387948\end{array}$

$\begin{array}{llll}0.2656165424053632 & 0.4660887398046609 & 0.1036435642353348\end{array}$

$\begin{array}{llll}0.0662827640724098 & 0.6668978417762262 & 0.1040741802220226\end{array}$

$\begin{array}{llll}0.2676980505153793 & 0.2672723431627677 & 0.1053281128480125\end{array}$

$\begin{array}{llll}0.8682111859075392 & 0.0655248638575283 & 0.1040161575402173\end{array}$

$\begin{array}{llll}0.8674100384779204 & 0.2666767970862946 & 0.1039196310430634\end{array}$

$\begin{array}{llll}0.4693637118310061 & 0.4658218027747754 & 0.1028647722261352\end{array}$

$\begin{array}{llll}0.6674442105810772 & 0.4685194117449911 & 0.1050089245476440\end{array}$

$\begin{array}{llll}0.4692627312642113 & 0.6676962545220507 & 0.1041931041926873\end{array}$

$\begin{array}{llll}0.6675151596868076 & 0.2650244583122361 & 0.1049668307842856\end{array}$

$\begin{array}{llll}0.0695383610089882 & 0.8655057036054163 & 0.1055291035802769\end{array}$

0.26672888943458220 .86577396401309530 .1051459840588735

$\begin{array}{llll}0.2654268909397144 & 0.6684606350503229 & 0.1037469083027273\end{array}$

$\begin{array}{llll}0.8681043134855044 & 0.4667786822185420 & 0.1040470354202202\end{array}$

$\begin{array}{llll}0.8673779707445426 & 0.6663608473184991 & 0.1040075447219194\end{array}$

$\begin{array}{llll}0.4672210149429779 & 0.8671415607408136 & 0.1041887698395313\end{array}$

$\begin{array}{llll}0.6678356536629906 & 0.8665400068918631 & 0.1038741461593683\end{array}$

$\begin{array}{llll}0.6674188522217780 & 0.6665929495158105 & 0.1048960078180737\end{array}$

$\begin{array}{llll}0.8676016924351364 & 0.8663765429422238 & 0.1039918807690052\end{array}$

$\begin{array}{llll}0.1995383963078504 & 0.0021686569233523 & 0.1564128305338141\end{array}$

$\begin{array}{llll}0.0013654549082679 & 0.1996626441287477 & 0.1566214640349679\end{array}$

$\begin{array}{llll}0.0020096315184074 & 0.9992678112836828 & 0.1567685406545812\end{array}$

0.60202016747380950 .99731318814017430 .1563669269017717

$\begin{array}{llll}0.1994771638690502 & 0.1987211834159248 & 0.1563940822575049\end{array}$

$\begin{array}{llll}0.4008908509036900 & 0.1977002362168170 & 0.1577063808483500\end{array}$

0.19990744638629280 .39861286238709890 .1599284800888263

$\begin{array}{llll}0.4004093367277710 & 0.0007975962537981 & 0.1567659450403805\end{array}$

$\begin{array}{llll}-0.0000060052991146 & 0.6000747927586619 & 0.1565804131352920\end{array}$

$\begin{array}{llll}0.0013463638541699 & 0.3993145739335215 & 0.1567652604974257\end{array}$

0.60635427123304820 .19280060254986580 .1583757378741332

$\begin{array}{lll}0.8059519121434190 & 0.1972173035285549 & 0.1559620889102981\end{array}$

$\begin{array}{llll}0.6071083286538320 & 0.3974562775908613 & 0.1552977171237150\end{array}$

$\begin{array}{llll}0.8045007075512159 & 0.9968567262305228 & 0.1563414319110911\end{array}$

$\begin{array}{llll}0.1974735599016062 & 0.6007139384850211 & 0.1546580607559242\end{array}$

$\begin{array}{llll}0.4023295447327739 & 0.5989639787274078 & 0.1520161390426234\end{array}$ 
0.20118161277242430 .80054486324609750 .1606042663097653

$\begin{array}{llll}0.4022491211261338 & 0.4016317349096116 & 0.1519104574253555\end{array}$

$\begin{array}{llll}0.0005178764099347 & 0.7995395205166241 & 0.1565828672590189\end{array}$

$\begin{array}{llll}0.6038927147162505 & 0.6038184217859787 & 0.1587452877644973\end{array}$

$\begin{array}{llll}0.8033044127482450 & 0.5995550149163877 & 0.1564244973298912\end{array}$

$\begin{array}{llll}0.6016305146237212 & 0.8024277603684156 & 0.1562834962994762\end{array}$

$\begin{array}{llll}0.8048717122274567 & 0.3984660757983211 & 0.1561473546606328\end{array}$

$\begin{array}{llll}0.4004616020006370 & 0.8046182495988065 & 0.1560921343813176\end{array}$

$\begin{array}{llll}0.8014157826582028 & 0.7995251428487460 & 0.1564672146810532\end{array}$

$\begin{array}{lll}0.3300443505726260 & 0.3407341069814759 & 0.2030295119656907\end{array}$

$\begin{array}{llll}0.3156943406742956 & 0.7273682485320409 & 0.2044470517599498\end{array}$

$\begin{array}{llll}0.5778857534531243 & 0.2213858230330147 & 0.2124559633138098\end{array}$

$\begin{array}{llll}0.5631598914415706 & 0.6130881171434436 & 0.2127922902110182\end{array}$

$\begin{array}{llll}0.5102963550972262 & 0.3496129511436850 & 0.2142358297049963\end{array}$

$\begin{array}{llll}0.3174568102085821 & 0.5364887949449583 & 0.2073909408498824\end{array}$

$\begin{array}{llll}0.3829979091743491 & 0.4069863717435110 & 0.2083586068799650\end{array}$

$\begin{array}{llll}0.5030212009156159 & 0.5449989483926287 & 0.2144547737083836\end{array}$

$\begin{array}{llll}0.5681751203949187 & 0.4182773322594909 & 0.2172346080059388\end{array}$

$\begin{array}{llll}0.3752342435486257 & 0.6080021700793071 & 0.2089360385624800\end{array}$

$\begin{array}{llll}0.6653170057467337 & 0.3725330948417939 & 0.2222928370695474\end{array}$

$\begin{array}{lllll}0.2184029560267956 & 0.5830403449164687 & 0.2094557679045964\end{array}$

$\begin{array}{llll}0.5064557859004399 & 0.7018879223830770 & 0.2189793823918683\end{array}$

$\begin{array}{llll}0.6426299683624938 & 0.5773477759378659 & 0.2257184983759110\end{array}$

$\begin{array}{llll}0.6606102840020186 & 0.1830447015191946 & 0.2237601688466026\end{array}$

$\begin{array}{llll}0.5290108442914484 & 0.1823359999057994 & 0.2202970227499629\end{array}$

\section{Zwitterion on $\mathrm{Cu}$ - VASP - optB88-vdW - Start T}

$\mathrm{Cu} \mathrm{O} \mathrm{N} \mathrm{C} \mathrm{H}$

1.00000000000000

$\begin{array}{lll}12.7810000000000006 & 0.0000000000000000 & 0.0000000000000000\end{array}$

$\begin{array}{lll}6.3905000000000003 & 11.0686699999999991 & 0.0000000000000000\end{array}$

$\begin{array}{llll}0.0000000000000000 & 0.0000000000000000 & 40.0000000000000000\end{array}$

$\begin{array}{lllll}100 & 2 & 2 & 6 & 6\end{array}$

Direct

0.20000000000000280 .00000000000000000 .0000000000000000

$\begin{array}{llll}0.0000000000000000 & 0.2000000000000028 & 0.0000000000000000\end{array}$

$0.0000000000000000 \quad 0.0000000000000000 \quad 0.0000000000000000$

$\begin{array}{lll}0.5999899999999982 & 0.0000000000000000 & 0.0000000000000000\end{array}$

$\begin{array}{llll}0.2000000000000028 & 0.2000000000000028 & 0.0000000000000000\end{array}$

$\begin{array}{llll}0.3999900000000025 & 0.2000000000000028 & 0.0000000000000000\end{array}$

0.20000000000000280 .39999000000000250 .0000000000000000

0.39999000000000250 .00000000000000000 .0000000000000000

$\begin{array}{llll}0.0000000000000000 & 0.5999899999999982 & 0.0000000000000000\end{array}$

$\begin{array}{lll}0.0000000000000000 & 0.3999900000000025 & 0.0000000000000000\end{array}$

$\begin{array}{lll}0.5999899999999982 & 0.2000000000000028 & 0.0000000000000000\end{array}$

$\begin{array}{llll}0.7999900000000011 & 0.2000000000000028 & 0.0000000000000000\end{array}$ 
0.59998999999999820 .39999000000000250 .0000000000000000

$\begin{array}{llll}0.7999900000000011 & 0.0000000000000000 & 0.0000000000000000\end{array}$

$\begin{array}{lll}0.2000000000000028 & 0.5999899999999982 & 0.0000000000000000\end{array}$

$\begin{array}{llll}0.3999900000000025 & 0.5999899999999982 & 0.0000000000000000\end{array}$

0.20000000000000280 .79999000000000110 .0000000000000000

$\begin{array}{llll}0.3999900000000025 & 0.3999900000000025 & 0.0000000000000000\end{array}$

$\begin{array}{lllll}0.0000000000000000 & 0.7999900000000011 & 0.0000000000000000\end{array}$

$\begin{array}{llll}0.5999899999999982 & 0.5999899999999982 & 0.0000000000000000\end{array}$

$\begin{array}{llll}0.7999900000000011 & 0.5999899999999982 & 0.0000000000000000\end{array}$

0.59998999999999820 .79999000000000110 .0000000000000000

$\begin{array}{lll}0.7999900000000011 & 0.3999900000000025 & 0.0000000000000000\end{array}$

$\begin{array}{llll}0.3999900000000025 & 0.7999900000000011 & 0.0000000000000000\end{array}$

$\begin{array}{llll}0.7999900000000011 & 0.7999900000000011 & 0.0000000000000000\end{array}$

$\begin{array}{llll}0.3333299999999966 & 0.1333300000000008 & 0.0521799999999999\end{array}$

$\begin{array}{llll}0.1333300000000008 & 0.3333299999999966 & 0.0521799999999999\end{array}$

$\begin{array}{llll}0.1333300000000008 & 0.1333300000000008 & 0.0521799999999999\end{array}$

$\begin{array}{llll}0.7333199999999991 & 0.1333300000000008 & 0.0521799999999999\end{array}$

$\begin{array}{llll}0.3333299999999966 & 0.3333299999999966 & 0.0521799999999999\end{array}$

$\begin{array}{lllll}0.5333299999999994 & 0.3333299999999966 & 0.0521799999999999\end{array}$

$\begin{array}{llll}0.3333299999999966 & 0.5333299999999994 & 0.0521799999999999\end{array}$

$\begin{array}{llll}0.5333299999999994 & 0.1333300000000008 & 0.0521799999999999\end{array}$

$\begin{array}{llll}0.1333300000000008 & 0.7333199999999991 & 0.0521799999999999\end{array}$

$\begin{array}{lll}0.1333300000000008 & 0.5333299999999994 & 0.0521799999999999\end{array}$

$\begin{array}{llll}0.7333199999999991 & 0.3333299999999966 & 0.0521799999999999\end{array}$

$\begin{array}{lllll}0.9333200000000019 & 0.3333299999999966 & 0.0521799999999999\end{array}$

$\begin{array}{llll}0.7333199999999991 & 0.5333299999999994 & 0.0521799999999999\end{array}$

$\begin{array}{llll}0.9333200000000019 & 0.1333300000000008 & 0.0521799999999999\end{array}$

$\begin{array}{llll}0.3333299999999966 & 0.7333199999999991 & 0.0521799999999999\end{array}$

$\begin{array}{llll}0.5333299999999994 & 0.7333199999999991 & 0.0521799999999999\end{array}$

$\begin{array}{llll}0.3333299999999966 & 0.9333200000000019 & 0.0521799999999999\end{array}$

$\begin{array}{llll}0.5333299999999994 & 0.5333299999999994 & 0.0521799999999999\end{array}$

$\begin{array}{llll}0.1333300000000008 & 0.9333200000000019 & 0.0521799999999999\end{array}$

$\begin{array}{llll}0.7333199999999991 & 0.7333199999999991 & 0.0521799999999999\end{array}$

$\begin{array}{llll}0.9333200000000019 & 0.7333199999999991 & 0.0521799999999999\end{array}$

$\begin{array}{lll}0.7333199999999991 & 0.9333200000000019 & 0.0521799999999999\end{array}$

0.93332000000000190 .53332999999999940 .0521799999999999

$\begin{array}{lll}0.5333299999999994 & 0.9333200000000019 & 0.0521799999999999\end{array}$

$\begin{array}{llll}0.9333200000000019 & 0.9333200000000019 & 0.0521799999999999\end{array}$

$\begin{array}{llll}0.0662644507798893 & 0.0669339020745106 & 0.1040416137492381\end{array}$

$\begin{array}{llll}0.2668080639464782 & 0.0670290151626714 & 0.1038614874345668\end{array}$

$\begin{array}{llll}0.0665985760316648 & 0.2672544164227450 & 0.1041871206754253\end{array}$

$\begin{array}{llll}0.4676430342571027 & 0.0668001832940132 & 0.1038087848780600\end{array}$

$\begin{array}{llll}0.6670308273308672 & 0.0663654750353878 & 0.1038162409579639\end{array}$

$\begin{array}{llll}0.4660417162455021 & 0.2678100442728049 & 0.1046005590809108\end{array}$

$\begin{array}{llll}0.0671200561999339 & 0.4669729973380685 & 0.1041820725109221\end{array}$

$0.2688284354222156 \quad 0.46676817431386940 .1052125023966968$

$\begin{array}{llll}0.0674135202755953 & 0.6665571202582988 & 0.1043332887392426\end{array}$ 
$\begin{array}{lll}0.2669034818777302 & 0.2673301274208870 & 0.1042246964314191\end{array}$

$\begin{array}{llll}0.8664137199578485 & 0.0663748242002256 & 0.1039020034900501\end{array}$

$\begin{array}{llll}0.8665213133137865 & 0.2670209462178848 & 0.1039547461587754\end{array}$

$\begin{array}{llll}0.4673361815907497 & 0.4652871582781024 & 0.1059841137014597\end{array}$

$\begin{array}{llll}0.6652702212640244 & 0.4658970102779970 & 0.1047162622808060\end{array}$

$\begin{array}{llll}0.4650140965662605 & 0.6688204489242311 & 0.1051764014427831\end{array}$

$\begin{array}{llll}0.6661829454808411 & 0.2674092976715325 & 0.1042906905663047\end{array}$

$\begin{array}{llll}0.0673797635038982 & 0.8669962666353148 & 0.1048136712268059\end{array}$

$\begin{array}{llll}0.2672200128787866 & 0.8663187403624596 & 0.1054551437658674\end{array}$

$\begin{array}{llll}0.2650106206023589 & 0.6694889773994077 & 0.1049271736370549\end{array}$

$\begin{array}{llll}0.8668832099349505 & 0.4671741166084801 & 0.1039509989197532\end{array}$

$\begin{array}{llll}0.8668707444730305 & 0.6666708168597115 & 0.1041141326742414\end{array}$

$\begin{array}{llll}0.4666001794487185 & 0.8666671205950781 & 0.1041371649570777\end{array}$

$\begin{array}{llll}0.6674308309387791 & 0.8668487872836851 & 0.1037924452967717\end{array}$

$\begin{array}{llll}0.6668815344634884 & 0.6663152888686703 & 0.1038283021316537\end{array}$

$\begin{array}{llll}0.8670309802944135 & 0.8664751412626652 & 0.1040561043389250\end{array}$

$\begin{array}{llll}0.1969984901923850 & 0.0074443278107089 & 0.1554231951862506\end{array}$

$\begin{array}{llll}0.9991337007734686 & 0.2023790132930278 & 0.1563961742682483\end{array}$

$\begin{array}{lll}0.9957401162173950 & 0.0049136703559935 & 0.1560960974940003\end{array}$

$\begin{array}{llll}0.6012512740828860 & 0.9984951644425393 & 0.1562257909835103\end{array}$

$\begin{array}{llll}0.1991027486384477 & 0.2018643244739944 & 0.1564086556415990\end{array}$

$\begin{array}{llll}0.4002450310459421 & 0.1964890204284188 & 0.1561143741500655\end{array}$

$\begin{array}{llll}0.1978339647841418 & 0.3999239580215495 & 0.1562597908163904\end{array}$

$\begin{array}{llll}0.4001785086209974 & 0.0036103922918605 & 0.1552844050024868\end{array}$

$\begin{array}{llll}0.9992532335978631 & 0.6004463493070586 & 0.1563205193168247\end{array}$

$\begin{array}{llll}0.9999875135693805 & 0.4010205373515680 & 0.1564148006708361\end{array}$

$\begin{array}{llll}0.6029315029906406 & 0.1945775398985820 & 0.1552106465065841\end{array}$

$\begin{array}{llll}0.8027193070459060 & 0.1978392352602683 & 0.1556442847089885\end{array}$

$\begin{array}{llll}0.6085695664004871 & 0.3930960042757466 & 0.1582610184247762\end{array}$

$\begin{array}{llll}0.8000887391985207 & 0.9992848725069856 & 0.1562763858069783\end{array}$

$\begin{array}{llll}0.1971131982959228 & 0.6018211961815634 & 0.1560476489843160\end{array}$

$\begin{array}{llll}0.3975622288189286 & 0.6016764738380859 & 0.1550731318352302\end{array}$

$\begin{array}{lll}0.1915878364716254 & 0.8116551942657522 & 0.1580436083398510\end{array}$

$\begin{array}{llll}0.4013712204931670 & 0.3905551925513970 & 0.1583584626539077\end{array}$

$\begin{array}{llll}0.9971088865361566 & 0.8011651356352807 & 0.1561089541880694\end{array}$

$\begin{array}{llll}0.6023873792815362 & 0.6002758789465212 & 0.1552258033888066\end{array}$

$\begin{array}{llll}0.8006053271655170 & 0.6000955711249715 & 0.1563832743248507\end{array}$

$\begin{array}{llll}0.6045580874761781 & 0.7996802055706251 & 0.1551557836269787\end{array}$

$\begin{array}{llll}0.8035916688657738 & 0.4002049031706713 & 0.1555923101898948\end{array}$

$\begin{array}{llll}0.4024973393797512 & 0.8080140148368327 & 0.1578777817688037\end{array}$

$\begin{array}{llll}0.8007372159317736 & 0.7997882479811994 & 0.1563988712125767\end{array}$

$\begin{array}{llll}0.4026467465875350 & 0.3929730679163133 & 0.2090974966981692\end{array}$

$\begin{array}{llll}0.1949076107539457 & 0.8261977234471735 & 0.2088653596444940\end{array}$

$\begin{array}{llll}0.6158564017686536 & 0.3834249365190748 & 0.2135825863335409\end{array}$

$\begin{array}{llll}0.4050323466470348 & 0.8240618656841628 & 0.2133126674889080\end{array}$

$\begin{array}{llll}0.5107890946794081 & 0.4961146046851525 & 0.2190719116662415\end{array}$

$\begin{array}{llll}0.2911542154885303 & 0.6098769655567333 & 0.2135756832132429\end{array}$ 
0.39935118842081990 .49635418177653990 .2146548840192755

$\begin{array}{llll}0.4059142486923019 & 0.7148559565949977 & 0.2192639337085267\end{array}$

$\begin{array}{llll}0.5133730053757875 & 0.6039204181251343 & 0.2219186384696662\end{array}$

$\begin{array}{llll}0.2919275330155622 & 0.7205997493962851 & 0.2147694955695884\end{array}$

$\begin{array}{llll}0.6000005246895205 & 0.6015649573895687 & 0.2241138534036077\end{array}$

$\begin{array}{llll}0.2039034140840814 & 0.6125523183121053 & 0.2133929030620738\end{array}$

$\begin{array}{llll}0.3246493461428429 & 0.8985519485750276 & 0.2197551192027618\end{array}$

$\begin{array}{llll}0.4786788330252356 & 0.8283584550281794 & 0.2213022616459273\end{array}$

$\begin{array}{llll}0.6949140605551456 & 0.3739303705509660 & 0.2225689756022174\end{array}$

$\begin{array}{llll}0.6030422710133665 & 0.3128501129878613 & 0.2198967694891349\end{array}$

Zwitterion on Cu - VASP - optB88-vdW - Start T30

$\mathrm{Cu} \mathrm{O} \mathrm{N} \mathrm{C} \mathrm{H}$

1.00000000000000

$\begin{array}{lll}12.7810000000000006 & 0.0000000000000000 & 0.0000000000000000\end{array}$

$\begin{array}{llll}6.3905000000000003 & 11.0686699999999991 & 0.0000000000000000\end{array}$

$\begin{array}{llll}0.0000000000000000 & 0.0000000000000000 & 40.0000000000000000\end{array}$

$\begin{array}{lllll}100 & 2 & 2 & 6 & 6\end{array}$

Direct

$\begin{array}{lll}0.2000000000000028 & 0.0000000000000000 & 0.0000000000000000\end{array}$

$\begin{array}{lllll}0.0000000000000000 & 0.2000000000000028 & 0.0000000000000000\end{array}$

$\begin{array}{llll}0.0000000000000000 & 0.0000000000000000 & 0.0000000000000000\end{array}$

$\begin{array}{lllll}0.5999899999999982 & 0.0000000000000000 & 0.0000000000000000\end{array}$

$\begin{array}{llll}0.2000000000000028 & 0.2000000000000028 & 0.0000000000000000\end{array}$

$\begin{array}{llll}0.3999900000000025 & 0.2000000000000028 & 0.0000000000000000\end{array}$

$\begin{array}{llll}0.2000000000000028 & 0.3999900000000025 & 0.0000000000000000\end{array}$

$\begin{array}{llll}0.3999900000000025 & 0.0000000000000000 & 0.0000000000000000\end{array}$

$\begin{array}{lllll}0.0000000000000000 & 0.5999899999999982 & 0.0000000000000000\end{array}$

$\begin{array}{llll}0.0000000000000000 & 0.3999900000000025 & 0.0000000000000000\end{array}$

$\begin{array}{llll}0.5999899999999982 & 0.2000000000000028 & 0.0000000000000000\end{array}$

$\begin{array}{lllll}0.7999900000000011 & 0.2000000000000028 & 0.0000000000000000\end{array}$

$\begin{array}{lllll}0.5999899999999982 & 0.3999900000000025 & 0.0000000000000000\end{array}$

$\begin{array}{lllll}0.7999900000000011 & 0.0000000000000000 & 0.0000000000000000\end{array}$

$\begin{array}{lllll}0.2000000000000028 & 0.5999899999999982 & 0.0000000000000000\end{array}$

$\begin{array}{lllll}0.3999900000000025 & 0.5999899999999982 & 0.0000000000000000\end{array}$

$\begin{array}{lllll}0.2000000000000028 & 0.7999900000000011 & 0.0000000000000000\end{array}$

$\begin{array}{lllll}0.3999900000000025 & 0.3999900000000025 & 0.0000000000000000\end{array}$

$\begin{array}{lllll}0.0000000000000000 & 0.7999900000000011 & 0.0000000000000000\end{array}$

$\begin{array}{lllll}0.5999899999999982 & 0.5999899999999982 & 0.0000000000000000\end{array}$

$\begin{array}{lllll}0.7999900000000011 & 0.5999899999999982 & 0.0000000000000000\end{array}$

$\begin{array}{lllll}0.5999899999999982 & 0.7999900000000011 & 0.0000000000000000\end{array}$

$\begin{array}{lllll}0.7999900000000011 & 0.3999900000000025 & 0.0000000000000000\end{array}$

$\begin{array}{lllll}0.3999900000000025 & 0.7999900000000011 & 0.0000000000000000\end{array}$

$\begin{array}{llllll}0.7999900000000011 & 0.7999900000000011 & 0.0000000000000000\end{array}$

$\begin{array}{lllll}0.3333299999999966 & 0.1333300000000008 & 0.0521799999999999\end{array}$

$\begin{array}{lllll}0.1333300000000008 & 0.3333299999999966 & 0.0521799999999999\end{array}$ 
$\begin{array}{llll}0.1333300000000008 & 0.1333300000000008 & 0.0521799999999999\end{array}$

$\begin{array}{llll}0.7333199999999991 & 0.1333300000000008 & 0.0521799999999999\end{array}$

$\begin{array}{lllll}0.3333299999999966 & 0.3333299999999966 & 0.0521799999999999\end{array}$

$\begin{array}{lllll}0.5333299999999994 & 0.3333299999999966 & 0.0521799999999999\end{array}$

$\begin{array}{llll}0.3333299999999966 & 0.5333299999999994 & 0.0521799999999999\end{array}$

$\begin{array}{llll}0.5333299999999994 & 0.1333300000000008 & 0.0521799999999999\end{array}$

$\begin{array}{llll}0.1333300000000008 & 0.7333199999999991 & 0.0521799999999999\end{array}$

$\begin{array}{llll}0.1333300000000008 & 0.5333299999999994 & 0.0521799999999999\end{array}$

$\begin{array}{llll}0.7333199999999991 & 0.3333299999999966 & 0.0521799999999999\end{array}$

$\begin{array}{llll}0.9333200000000019 & 0.3333299999999966 & 0.0521799999999999\end{array}$

$\begin{array}{lll}0.7333199999999991 & 0.5333299999999994 & 0.0521799999999999\end{array}$

$\begin{array}{llll}0.9333200000000019 & 0.1333300000000008 & 0.0521799999999999\end{array}$

$\begin{array}{llll}0.3333299999999966 & 0.7333199999999991 & 0.0521799999999999\end{array}$

$\begin{array}{llll}0.5333299999999994 & 0.7333199999999991 & 0.0521799999999999\end{array}$

$\begin{array}{llll}0.3333299999999966 & 0.9333200000000019 & 0.0521799999999999\end{array}$

$\begin{array}{llll}0.5333299999999994 & 0.5333299999999994 & 0.0521799999999999\end{array}$

$\begin{array}{llll}0.1333300000000008 & 0.9333200000000019 & 0.0521799999999999\end{array}$

$\begin{array}{llll}0.7333199999999991 & 0.7333199999999991 & 0.0521799999999999\end{array}$

$\begin{array}{llll}0.9333200000000019 & 0.7333199999999991 & 0.0521799999999999\end{array}$

$\begin{array}{llll}0.7333199999999991 & 0.9333200000000019 & 0.0521799999999999\end{array}$

$\begin{array}{llll}0.9333200000000019 & 0.5333299999999994 & 0.0521799999999999\end{array}$

$\begin{array}{lll}0.5333299999999994 & 0.9333200000000019 & 0.0521799999999999\end{array}$

$\begin{array}{llll}0.9333200000000019 & 0.9333200000000019 & 0.0521799999999999\end{array}$

$\begin{array}{llll}0.0681682852539892 & 0.0642328809561110 & 0.1057962647016156\end{array}$

$\begin{array}{llll}0.2643577293200460 & 0.0646416639063237 & 0.1051677978452855\end{array}$

$\begin{array}{llll}0.0653930890917130 & 0.2656457726212583 & 0.1043686173212762\end{array}$

$\begin{array}{llll}0.4660456513254302 & 0.0664271451308921 & 0.1039724133356677\end{array}$

$\begin{array}{llll}0.6662705155535086 & 0.0653916419426247 & 0.1039611338292249\end{array}$

$\begin{array}{llll}0.4648641340862147 & 0.2671914466053394 & 0.1049991078613296\end{array}$

$\begin{array}{llll}0.0651080801756509 & 0.4657247963564384 & 0.1042646795904712\end{array}$

$\begin{array}{llll}0.2654777853712834 & 0.4660498255025610 & 0.1052842058727443\end{array}$

$\begin{array}{llll}0.0673840034370472 & 0.6639411098255288 & 0.1055000686245690\end{array}$

$\begin{array}{llll}0.2656720568835997 & 0.2651724701972422 & 0.1042120544537045\end{array}$

$\begin{array}{llll}0.8661193413382346 & 0.0654886076263361 & 0.1041150379887935\end{array}$

$\begin{array}{llll}0.8666129519740696 & 0.2647277973862637 & 0.1042311394628733\end{array}$

$\begin{array}{llll}0.4673546550483989 & 0.4629360121412091 & 0.1048048364730248\end{array}$

$\begin{array}{llll}0.6652587388727486 & 0.4640969785888510 & 0.1051126175419668\end{array}$

$\begin{array}{llll}0.4669283005303103 & 0.6651061846344900 & 0.1031474464794906\end{array}$

$\begin{array}{llll}0.6659487890337140 & 0.2656626362938644 & 0.1049383669467703\end{array}$

$\begin{array}{llll}0.0648117510438926 & 0.8656480314430205 & 0.1042102639098016\end{array}$

$\begin{array}{llll}0.2634822544799651 & 0.8673133852504986 & 0.1040671886256136\end{array}$

$\begin{array}{llll}0.2637764552825548 & 0.6648301618262896 & 0.1035985961579088\end{array}$

0.86500185544489260 .46611243625506330 .1041701986151105

$\begin{array}{llll}0.8655989292991629 & 0.6659306949168609 & 0.1042133563033497\end{array}$

$\begin{array}{llll}0.4673780215179758 & 0.8663370680457655 & 0.1042541686625471\end{array}$

$\begin{array}{llll}0.6649438331224476 & 0.8652149935781128 & 0.1052734477695006\end{array}$

$\begin{array}{llll}0.6647079946221246 & 0.6675966299803895 & 0.1053183881028184\end{array}$ 
$\begin{array}{llll}0.8652584110552376 & 0.8652939139844101 & 0.1041192121577922\end{array}$

$\begin{array}{llll}0.1994788905588050 & 0.9971998951699298 & 0.1614484024101971\end{array}$

$\begin{array}{llll}0.9991942496665486 & 0.1978213248616466 & 0.1565762957458476\end{array}$

$\begin{array}{llll}0.9980186997799092 & 0.9975382992448200 & 0.1563355920815784\end{array}$

$\begin{array}{llll}0.5989677588412260 & 0.0000467968473474 & 0.1562596782506780\end{array}$

$\begin{array}{lllll}0.1970211198328057 & 0.1992079457487126 & 0.1562883725720878\end{array}$

$\begin{array}{llll}0.3975851962224505 & 0.1988485314956682 & 0.1565222250739953\end{array}$

$\begin{array}{llll}0.1951207966192540 & 0.3957353536425077 & 0.1559379696919053\end{array}$

$\begin{array}{lll}0.3982942002613732 & 0.0033348553803208 & 0.1549129074232006\end{array}$

$\begin{array}{llll}0.9961083936233531 & 0.5977280637106785 & 0.1564440839800978\end{array}$

$\begin{array}{llll}0.9971932066761210 & 0.3981826362212565 & 0.1563370507804460\end{array}$

$\begin{array}{lllll}0.5990636726365778 & 0.1952766623468954 & 0.1560517388070936\end{array}$

$\begin{array}{llll}0.8017179370211946 & 0.1957690393387440 & 0.1561292907286898\end{array}$

$\begin{array}{llll}0.6015532696054688 & 0.3910939227323433 & 0.1583965316974222\end{array}$

$\begin{array}{llll}0.7986015879990527 & 0.9980890853456338 & 0.1561257221229720\end{array}$

$\begin{array}{llll}0.1938348494186830 & 0.5956079386498648 & 0.1592266303902313\end{array}$

$\begin{array}{llll}0.3982949569100991 & 0.6009005607928835 & 0.1520442941676095\end{array}$

$\begin{array}{llll}0.1948815395553148 & 0.7968221528719830 & 0.1547366621455395\end{array}$

0.39586260496216230 .39626274012030040 .1574535123838467

$\begin{array}{llll}0.9959428290855230 & 0.7986537704065910 & 0.1562750570875646\end{array}$

$\begin{array}{llll}0.6021008516147727 & 0.5956776680382092 & 0.1554905051621170\end{array}$

$\begin{array}{llll}0.7993118453659778 & 0.5979828616197794 & 0.1562939775812507\end{array}$

$\begin{array}{llll}0.5977542965963315 & 0.8020927503909548 & 0.1598925071201532\end{array}$

$\begin{array}{llll}0.8012764542701568 & 0.3964892573910301 & 0.1560750827709999\end{array}$

$\begin{array}{llll}0.3993866711298766 & 0.7985040685720418 & 0.1523765447790606\end{array}$

$\begin{array}{lllll}0.7987748553426909 & 0.7981133971819174 & 0.1563969300522737\end{array}$

$\begin{array}{llll}0.3154173477377864 & 0.5303839482528224 & 0.2020442256852116\end{array}$

$\begin{array}{llll}0.2906223017748261 & 0.9216475563108294 & 0.2069089996748739\end{array}$

$\begin{array}{llll}0.5625961954817499 & 0.4156583775042871 & 0.2122895669132041\end{array}$

$\begin{array}{llll}0.5383787292454543 & 0.8120327707369291 & 0.2140621053142024\end{array}$

$\begin{array}{llll}0.4926190239365505 & 0.5438029067739237 & 0.2140958365565962\end{array}$

0.29744086526289340 .72840698112970050 .2074903413227656

$\begin{array}{llll}0.3658892188535303 & 0.5989195021247197 & 0.2079434387151944\end{array}$

$\begin{array}{llll}0.4809940335294426 & 0.7411367087668470 & 0.2149092752933657\end{array}$

$\begin{array}{llll}0.5481810607840254 & 0.6145893878197261 & 0.2175169934935706\end{array}$

$\begin{array}{llll}0.3524546908976137 & 0.8024079554013613 & 0.2099392245246284\end{array}$

$\begin{array}{llll}0.6451659695251480 & 0.5701259364318610 & 0.2225609942248380\end{array}$

$0.19869421549168630 .7722548014023304 \quad 0.2099346256283247$

$\begin{array}{llll}0.4770494478872060 & 0.9007156803374773 & 0.2192011025214339\end{array}$

$\begin{array}{llll}0.6132976922299364 & 0.7805881383053127 & 0.2288151487169191\end{array}$

$\begin{array}{llll}0.6422136211465911 & 0.3783451725010812 & 0.2251495220056139\end{array}$

$\begin{array}{llll}0.5129936432173521 & 0.3756430158337379 & 0.2185346966883384\end{array}$

$\underline{\text { Zwitterion on Smaller Cu slab - VASP - optB88-vdW }}$

$\mathrm{Cu} \mathrm{O} \mathrm{N} \mathrm{C} \mathrm{H}$

1.00000000000000 


$\begin{array}{ccc}10.22499999999999996 & 0.0000000000000000 & 0.0000000000000000 \\ 0.0000000000000000 & 8.9190000000000005 & 0.0000000000000000 \\ 0.0000000000000000 & 0.0000000000000000 & 25.0000000000000000 \\ 48 & 6 & \end{array}$

Direct

$\begin{array}{llll}0.3748111251302365 & 0.0808372749893275 & 0.1668193368664271\end{array}$

$\begin{array}{lllll}0.6256157448270230 & 0.0802556624519066 & 0.1674571675100417\end{array}$

$\begin{array}{lllll}0.3749297414390593 & 0.5817289430047341 & 0.16653443333943118\end{array}$

$\begin{array}{lllll}0.6249596506798958 & 0.5807483412908940 & 0.1668959742547380\end{array}$

$\begin{array}{lllll}0.0000747382932237 & 0.8309632603092104 & 0.1664378131033341\end{array}$

$\begin{array}{lllll}0.9996270855719308 & 0.3309067949548027 & 0.1667638461435545\end{array}$

$\begin{array}{lllll}0.1247992603145931 & 0.0806958776904611 & 0.1665387978506649\end{array}$

$\begin{array}{llll}0.1249289761096856 & 0.5814322668832453 & 0.1667929587855395\end{array}$

$\begin{array}{lllll}0.2500086387206485 & 0.8312047226383548 & 0.1664893123627881\end{array}$

$\begin{array}{llll}0.2495111172442089 & 0.3314075792171153 & 0.1672239521516950\end{array}$

$\begin{array}{lllll}0.7502387118743989 & 0.8304747332031829 & 0.1665333143039707\end{array}$

$\begin{array}{lllll}0.8752428756210969 & 0.0806139322904192 & 0.1665448281889056\end{array}$

$\begin{array}{llll}0.7502146114188530 & 0.3308797538633559 & 0.1666895778458763\end{array}$

$\begin{array}{lllll}0.4999540199366401 & 0.8309007288955842 & 0.1665635425120986\end{array}$

$\begin{array}{lllll}0.8749532783720220 & 0.5808646420844049 & 0.16665556784142849\end{array}$

$\begin{array}{lllll}0.4997898219188604 & 0.3309082614982432 & 0.1670678890390141\end{array}$

$\begin{array}{lllll}0.5002141290655731 & 0.1642603630232597 & 0.0838424995206603\end{array}$

$\begin{array}{lllll}0.5000953204652647 & 0.6641870168001320 & 0.0833544287702540\end{array}$

$\begin{array}{lllll}0.2501217908945937 & 0.1647664098766871 & 0.0836406724484822\end{array}$

$\begin{array}{lllll}0.6247933075517836 & 0.4142015327757990 & 0.0835923408369772\end{array}$

$\begin{array}{lllll}0.3749164503411959 & 0.4141224618864087 & 0.0837309921041705\end{array}$

$\begin{array}{lllll}0.2499256253682650 & 0.6642507281534644 & 0.0833161547543191\end{array}$

$\begin{array}{lllll}0.7497328710367398 & 0.1642710507891120 & 0.0835911204711033\end{array}$

$\begin{array}{lllll}0.3750835804666998 & 0.9143216055190762 & 0.0830500797662045\end{array}$

$\begin{array}{lllll}0.1250212864422005 & 0.4143680169569711 & 0.0836216605625301\end{array}$

$\begin{array}{lllll}0.6250589219378739 & 0.9142933254407687 & 0.0832009825045329\end{array}$

$\begin{array}{llll}0.1250343162135632 & 0.9142624649597508 & 0.0829229108094470\end{array}$

$\begin{array}{lllll}0.9999538989045554 & 0.1646075031393782 & 0.0833694779748021\end{array}$

$\begin{array}{llll}0.7498788450196823 & 0.6640441163231738 & 0.0833537269055654\end{array}$

$\begin{array}{llll}0.9999846938237568 & 0.6640872154462659 & 0.08333300569855265\end{array}$

$\begin{array}{llll}0.8750005169128735 & 0.9140683360962085 & 0.0829628487025133\end{array}$

$\begin{array}{lllll}0.8749017741337851 & 0.4143219985424522 & 0.0833783949960345\end{array}$

$\begin{array}{llll}0.9999800000000008 & 0.4963899999999981 & 0.0000000000000000\end{array}$

$\begin{array}{llll}0.1249699999999976 & 0.7446100000000015 & 0.0000000000000000\end{array}$

$\begin{array}{lllll}0.3749899999999968 & 0.2481999999999971 & 0.0000000000000000\end{array}$

$\begin{array}{lllll}0.2499800000000008 & 0.4963899999999981 & 0.0000000000000000\end{array}$

$\begin{array}{llllll}0.1249899999999968 & 0.2481999999999971 & 0.0000000000000000\end{array}$

$\begin{array}{lllll}0.8749600000000015 & 0.2481999999999971 & 0.0000000000000000\end{array}$

$\begin{array}{lllll}0.4999800000000008 & 0.4963899999999981 & 0.0000000000000000\end{array}$

$\begin{array}{llll}0.7499699999999976 & 0.4963899999999981 & 0.00000000000000000\end{array}$

$\begin{array}{llll}0.6249699999999976 & 0.7446100000000015 & 0.0000000000000000\end{array}$

$\begin{array}{lllll}0.6249800000000008 & 0.2481999999999971 & 0.0000000000000000\end{array}$ 
$\begin{array}{lll}0.3749800000000008 & 0.7446100000000015 & 0.0000000000000000\end{array}$

$\begin{array}{llll}0.8749699999999976 & 0.7446100000000015 & 0.0000000000000000\end{array}$

$\begin{array}{llll}0.9999899999999968 & 0.0000000000000000 & 0.0000000000000000\end{array}$

$\begin{array}{lll}0.2499899999999968 & 0.0000000000000000 & 0.0000000000000000\end{array}$

$\begin{array}{lll}0.4999800000000008 & 0.0000000000000000 & 0.0000000000000000\end{array}$

$\begin{array}{lllll}0.7499699999999976 & 0.0000000000000000 & 0.0000000000000000\end{array}$

$\begin{array}{lllll}0.6091567726308609 & 0.0717781974112464 & 0.2996292071443777\end{array}$

$\begin{array}{llll}0.2386329784934306 & 0.3945865702245237 & 0.3024345207470968\end{array}$

$\begin{array}{llll}0.7639771007437276 & 0.3005229436471572 & 0.3146860805183130\end{array}$

$\begin{array}{llll}0.3901451217981035 & 0.6268710192843736 & 0.3150055276066362\end{array}$

$\begin{array}{llll}0.5864001053152524 & 0.4776970068935599 & 0.3174754626989822\end{array}$

$\begin{array}{llll}0.4515043969346168 & 0.4960249303715990 & 0.3132956273445517\end{array}$

0.63764645295873320 .33356813549884850 .3128079049533858

$\begin{array}{llll}0.3584330576553319 & 0.3633053586843760 & 0.3051835064469013\end{array}$

$\begin{array}{llll}0.5508617693953570 & 0.1957729390493481 & 0.3038901419907014\end{array}$

$\begin{array}{llll}0.4151302882575867 & 0.2199072967441435 & 0.3006483132823144\end{array}$

$\begin{array}{llll}0.6503939229251071 & 0.5745511309654815 & 0.3233374795110344\end{array}$

$\begin{array}{llll}0.8356789094827481 & 0.3787360594794748 & 0.3184764710539445\end{array}$

$\begin{array}{llll}0.4372341880726438 & 0.7271238240077891 & 0.3176155739788209\end{array}$

$\begin{array}{llll}0.7865995000449629 & 0.1893877091953983 & 0.3093691374341907\end{array}$

$\begin{array}{llll}0.2904699859341151 & 0.6211488134316485 & 0.3103791359244712\end{array}$

$\begin{array}{llll}0.3507770892094456 & 0.1232577130875054 & 0.2952827975320493\end{array}$

Zwitterion on Another Smaller Slab - VASP - optB88-vdW

$\mathrm{Cu} \mathrm{O} \mathrm{N} \mathrm{C} \mathrm{H}$

1.00000000000000

$\begin{array}{lll}10.2249999999999996 & 0.0000000000000000 & 0.0000000000000000\end{array}$

$\begin{array}{lll}0.0000000000000000 & 11.2059999999999995 & 0.0000000000000000\end{array}$

$\begin{array}{llll}0.0000000000000000 & 0.0000000000000000 & 25.0000000000000000\end{array}$

$\begin{array}{lllll}60 & 2 & 2 & 6 & 6\end{array}$

Direct

$\begin{array}{lll}0.0000799999999970 & 0.6613800000000012 & 0.1664400000000015\end{array}$

$\begin{array}{lll}0.7499699999999976 & 0.7959099999999992 & 0.0000000000000000\end{array}$

$\begin{array}{lll}0.4999800000000008 & 0.7959099999999992 & 0.0000000000000000\end{array}$

$\begin{array}{llll}0.2499899999999968 & 0.7959099999999992 & 0.0000000000000000\end{array}$

$\begin{array}{llll}0.9999899999999968 & 0.7959099999999992 & 0.0000000000000000\end{array}$

$\begin{array}{lll}0.6249800000000008 & 0.9934599999999989 & 0.0000000000000000\end{array}$

$\begin{array}{lll}0.8749600000000015 & 0.9934599999999989 & 0.0000000000000000\end{array}$

$\begin{array}{llll}0.1249899999999968 & 0.9934599999999989 & 0.0000000000000000\end{array}$

$\begin{array}{llll}0.3749899999999968 & 0.9934599999999989 & 0.0000000000000000\end{array}$

$\begin{array}{llll}0.9999600000000015 & 0.9269299999999987 & 0.0833700000000022\end{array}$

$\begin{array}{lll}0.7497400000000027 & 0.9266599999999983 & 0.0835900000000009\end{array}$

$\begin{array}{lll}0.2501200000000026 & 0.9270500000000013 & 0.0836400000000026\end{array}$

$\begin{array}{llll}0.5002100000000027 & 0.9266500000000022 & 0.0838400000000021\end{array}$

$\begin{array}{llll}0.8752399999999980 & 0.8600799999999964 & 0.1665500000000009\end{array}$

0.12480999999999650 .86014000000000120 .1665399999999977

$\begin{array}{llll}0.6256199999999978 & 0.8597899999999967 & 0.1674599999999984\end{array}$ 
0.37480999999999650 .86025000000000060 .1668200000000013

$\begin{array}{llll}0.8749699999999976 & 0.5926499999999990 & 0.0000000000000000\end{array}$

$\begin{array}{llll}0.3749800000000008 & 0.5926499999999990 & 0.0000000000000000\end{array}$

$\begin{array}{lll}0.6249800000000008 & 0.1975499999999997 & 0.0000000000000000\end{array}$

$\begin{array}{lll}0.6249800000000008 & 0.5926499999999990 & 0.0000000000000000\end{array}$

$\begin{array}{llll}0.7499699999999976 & 0.3950800000000001 & 0.0000000000000000\end{array}$

$\begin{array}{lllll}0.4999800000000008 & 0.3950800000000001 & 0.0000000000000000\end{array}$

$\begin{array}{llll}0.8749600000000015 & 0.1975499999999997 & 0.0000000000000000\end{array}$

$\begin{array}{lll}0.1249899999999968 & 0.1975499999999997 & 0.0000000000000000\end{array}$

$\begin{array}{lll}0.2499800000000008 & 0.3950800000000001 & 0.0000000000000000\end{array}$

$\begin{array}{llll}0.3749899999999968 & 0.1975499999999997 & 0.0000000000000000\end{array}$

$\begin{array}{llll}0.1249800000000008 & 0.5926499999999990 & 0.0000000000000000\end{array}$

$\begin{array}{llll}0.9999800000000008 & 0.3950800000000001 & 0.0000000000000000\end{array}$

$\begin{array}{llll}0.8748999999999967 & 0.3297600000000003 & 0.0833799999999982\end{array}$

$\begin{array}{llll}0.8750000000000000 & 0.7275199999999984 & 0.0829599999999999\end{array}$

$\begin{array}{llll}0.9999899999999968 & 0.5285599999999988 & 0.0833299999999966\end{array}$

$\begin{array}{llll}0.7498799999999974 & 0.5285200000000003 & 0.0833500000000029\end{array}$

$\begin{array}{llll}0.9999600000000015 & 0.1310100000000034 & 0.0833700000000022\end{array}$

0.12503000000000240 .72767999999999940 .0829200000000014

$\begin{array}{llll}0.6250500000000017 & 0.7276999999999987 & 0.0831999999999979\end{array}$

$\begin{array}{llll}0.1250199999999992 & 0.3297999999999988 & 0.0836200000000034\end{array}$

$\begin{array}{llll}0.3750799999999970 & 0.7277199999999979 & 0.0830500000000001\end{array}$

$\begin{array}{llll}0.7497400000000027 & 0.1307499999999990 & 0.0835900000000009\end{array}$

0.24992999999999910 .52868999999999740 .0833200000000005

$\begin{array}{lll}0.3749099999999999 & 0.3296100000000024 & 0.0837300000000027\end{array}$

$\begin{array}{llll}0.6248000000000005 & 0.3296700000000001 & 0.0835900000000009\end{array}$

$\begin{array}{llll}0.2501200000000026 & 0.1311400000000020 & 0.0836400000000026\end{array}$

$\begin{array}{llll}0.5000900000000001 & 0.5286400000000029 & 0.0833599999999990\end{array}$

$\begin{array}{llll}0.5002100000000027 & 0.1307400000000030 & 0.0838400000000021\end{array}$

$\begin{array}{llll}0.4997899999999973 & 0.2633799999999979 & 0.1670700000000025\end{array}$

$\begin{array}{llll}0.8749600000000015 & 0.4623199999999983 & 0.1666600000000003\end{array}$

0.49996000000000150 .66132999999999950 .1665599999999969

$\begin{array}{llll}0.7502100000000027 & 0.2633500000000026 & 0.1666900000000027\end{array}$

$\begin{array}{llll}0.8752399999999980 & 0.0641600000000011 & 0.1665500000000009\end{array}$

$\begin{array}{llll}0.7502399999999980 & 0.6609899999999982 & 0.1665300000000016\end{array}$

$\begin{array}{llll}0.2495100000000008 & 0.2637700000000009 & 0.1672200000000004\end{array}$

0.25001000000000320 .66156999999999750 .1664900000000031

$\begin{array}{llll}0.1249299999999991 & 0.4627699999999990 & 0.1667899999999989\end{array}$

$\begin{array}{llll}0.1248099999999965 & 0.0642300000000020 & 0.1665399999999977\end{array}$

$\begin{array}{llll}0.9996300000000033 & 0.2633700000000019 & 0.1667599999999965\end{array}$

$\begin{array}{llll}0.6249600000000015 & 0.4622299999999981 & 0.1668999999999983\end{array}$

$\begin{array}{llll}0.3749400000000023 & 0.4630099999999970 & 0.1665399999999977\end{array}$

$\begin{array}{llll}0.6256199999999978 & 0.0638799999999975 & 0.1674599999999984\end{array}$

$\begin{array}{llll}0.3748099999999965 & 0.0643400000000014 & 0.1668200000000013\end{array}$

$\begin{array}{llll}0.2366861667786821 & 0.2996440397032032 & 0.2516307101536555\end{array}$

$\begin{array}{llll}0.6250197480508177 & 0.0604411860907277 & 0.2496503082889063\end{array}$

$\begin{array}{llll}0.3767020585615768 & 0.4946739430775722 & 0.2807321883283436\end{array}$ 
$\begin{array}{lll}0.7716537774076002 & 0.2552726996683958 & 0.2603459515434068\end{array}$

$\begin{array}{llll}0.4249361065732487 & 0.1727559695597733 & 0.2576133293465190\end{array}$

$\begin{array}{llll}0.5628701177177504 & 0.1605658143531201 & 0.2604518046861557\end{array}$

$\begin{array}{llll}0.3610530002500880 & 0.2850486106697616 & 0.2623401078654055\end{array}$

$\begin{array}{llll}0.6379579585958837 & 0.2671005070028158 & 0.2701769448499718\end{array}$

$\begin{array}{llll}0.4407233578931269 & 0.3883575408673607 & 0.2756952689507273\end{array}$

$\begin{array}{llll}0.5773992647469086 & 0.3773784262911022 & 0.2789917952410548\end{array}$

$\begin{array}{llll}0.3639568490842322 & 0.0921235101041020 & 0.2553798183853999\end{array}$

$\begin{array}{lll}0.2788930095229532 & 0.4947002004660490 & 0.2726313882126092\end{array}$

$\begin{array}{llll}0.8053672657474280 & 0.1703941406605551 & 0.2666902294725944\end{array}$

$\begin{array}{llll}0.4256593845920662 & 0.5734411346212909 & 0.2777015945866950\end{array}$

$\begin{array}{llll}0.8323961495779386 & 0.3209280153564933 & 0.2736997900390250\end{array}$

$\begin{array}{llll}0.6371226996324850 & 0.4568242926641162 & 0.2861614756128645\end{array}$

Zwitterion Hydrogen Bond Network Low Coverage - VASP- DFT-D2

$\mathrm{Cu} \mathrm{O} \mathrm{N} \mathrm{C} \mathrm{H}$

1.00000000000000

$\begin{array}{lll}15.3369999999999997 & 0.0000000000000000 & 0.0000000000000000\end{array}$

$\begin{array}{lll}7.6684999999999999 & 13.2822300000000002 & 0.0000000000000000\end{array}$

$\begin{array}{llll}0.0000000000000000 & 0.0000000000000000 & 40.0000000000000000\end{array}$

$\begin{array}{lllll}150 & 4 & 4 & 12 & 12\end{array}$

Direct

0.83333999999999970 .66667000000000340 .0000000000000000

0.00001000000000320 .66667000000000340 .0000000000000000

$\begin{array}{lll}0.0000100000000032 & 0.5000000000000000 & 0.0000000000000000\end{array}$

$\begin{array}{lllll}0.8333399999999997 & 0.3333299999999966 & 0.0000000000000000\end{array}$

$\begin{array}{llll}0.8333399999999997 & 0.5000000000000000 & 0.0000000000000000\end{array}$

0.00001000000000320 .33332999999999660 .0000000000000000

0.00001000000000320 .16667000000000340 .0000000000000000

$\begin{array}{llll}0.8333399999999997 & 0.0000000000000000 & 0.0000000000000000\end{array}$

$\begin{array}{lllll}0.8333399999999997 & 0.1666700000000034 & 0.0000000000000000\end{array}$

$\begin{array}{lll}0.0000100000000032 & 0.0000000000000000 & 0.0000000000000000\end{array}$

0.66667000000000340 .66667000000000340 .0000000000000000

0.33332999999999660 .66667000000000340 .0000000000000000

0.66667000000000340 .33332999999999660 .0000000000000000

$0.5000000000000000 \quad 0.66667000000000340 .0000000000000000$

0.66667000000000340 .50000000000000000 .0000000000000000

$\begin{array}{lll}0.5000000000000000 & 0.5000000000000000 & 0.0000000000000000\end{array}$

$0.0000000000000000 \quad 0.66667000000000340 .0000000000000000$

$\begin{array}{lll}0.3333299999999966 & 0.3333299999999966 & 0.0000000000000000\end{array}$

0.16667000000000340 .66667000000000340 .0000000000000000

0.33332999999999660 .50000000000000000 .0000000000000000

0.16667000000000340 .50000000000000000 .0000000000000000

$0.66667000000000340 .0000000000000000 \quad 0.0000000000000000$

$\begin{array}{llll}0.5000000000000000 & 0.3333299999999966 & 0.0000000000000000\end{array}$

0.66667000000000340 .16667000000000340 .0000000000000000

$\begin{array}{lll}0.5000000000000000 & 0.1666700000000034 & 0.0000000000000000\end{array}$ 
$\begin{array}{lll}0.0000000000000000 & 0.3333299999999966 & 0.0000000000000000\end{array}$

$\begin{array}{llll}0.0000000000000000 & 0.5000000000000000 & 0.0000000000000000\end{array}$

$\begin{array}{llll}0.3333299999999966 & 0.0000000000000000 & 0.0000000000000000\end{array}$

0.16667000000000340 .33332999999999660 .0000000000000000

0.33332999999999660 .16667000000000340 .0000000000000000

0.16667000000000340 .16667000000000340 .0000000000000000

$\begin{array}{llll}0.5000000000000000 & 0.0000000000000000 & 0.0000000000000000\end{array}$

$0.0000000000000000 \quad 0.0000000000000000 \quad 0.0000000000000000$

$0.0000000000000000 \quad 0.16667000000000340 .0000000000000000$

0.16667000000000340 .00000000000000000 .0000000000000000

$\begin{array}{lll}0.9444500000000033 & 0.7777799999999999 & 0.0521799999999999\end{array}$

$\begin{array}{llll}0.9444500000000033 & 0.4444500000000033 & 0.0521799999999999\end{array}$

0.94445000000000330 .61110999999999650 .0521799999999999

$\begin{array}{lll}0.9444500000000033 & 0.1111099999999965 & 0.0521799999999999\end{array}$

$\begin{array}{llll}0.9444500000000033 & 0.2777799999999999 & 0.0521799999999999\end{array}$

$\begin{array}{llll}0.7777799999999999 & 0.7777799999999999 & 0.0521799999999999\end{array}$

$\begin{array}{lll}0.4444500000000033 & 0.7777799999999999 & 0.0521799999999999\end{array}$

$\begin{array}{llll}0.7777799999999999 & 0.4444500000000033 & 0.0521799999999999\end{array}$

$\begin{array}{llll}0.6111099999999965 & 0.7777799999999999 & 0.0521799999999999\end{array}$

$\begin{array}{llll}0.7777799999999999 & 0.6111099999999965 & 0.0521799999999999\end{array}$

$\begin{array}{llll}0.6111099999999965 & 0.6111099999999965 & 0.0521799999999999\end{array}$

$\begin{array}{llll}0.1111099999999965 & 0.7777799999999999 & 0.0521799999999999\end{array}$

0.44445000000000330 .44445000000000330 .0521799999999999

$\begin{array}{llll}0.2777799999999999 & 0.7777799999999999 & 0.0521799999999999\end{array}$

$\begin{array}{llll}0.4444500000000033 & 0.6111099999999965 & 0.0521799999999999\end{array}$

$\begin{array}{lllll}0.2777799999999999 & 0.6111099999999965 & 0.0521799999999999\end{array}$

$\begin{array}{llll}0.7777799999999999 & 0.1111099999999965 & 0.0521799999999999\end{array}$

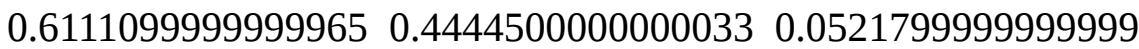

$\begin{array}{llll}0.7777799999999999 & 0.2777799999999999 & 0.0521799999999999\end{array}$

$\begin{array}{llll}0.6111099999999965 & 0.2777799999999999 & 0.0521799999999999\end{array}$

$\begin{array}{lllll}0.1111099999999965 & 0.4444500000000033 & 0.0521799999999999\end{array}$

$\begin{array}{llll}0.1111099999999965 & 0.6111099999999965 & 0.0521799999999999\end{array}$

$\begin{array}{llll}0.4444500000000033 & 0.1111099999999965 & 0.0521799999999999\end{array}$

$\begin{array}{llll}0.2780399999999972 & 0.4406099999999995 & 0.0521799999999999\end{array}$

$\begin{array}{lll}0.4444500000000033 & 0.2777799999999999 & 0.0521799999999999\end{array}$

$\begin{array}{llll}0.2777799999999999 & 0.2777799999999999 & 0.0521799999999999\end{array}$

$\begin{array}{llll}0.6111099999999965 & 0.1111099999999965 & 0.0521799999999999\end{array}$

$\begin{array}{llll}0.1111099999999965 & 0.1111099999999965 & 0.0521799999999999\end{array}$

$\begin{array}{llll}0.1111099999999965 & 0.2777799999999999 & 0.0521799999999999\end{array}$

$\begin{array}{llll}0.2777799999999999 & 0.1111099999999965 & 0.0521799999999999\end{array}$

$\begin{array}{llll}0.3885227974104628 & 0.5544306136540970 & 0.1037455936717478\end{array}$

$\begin{array}{llll}0.3867180755810479 & 0.3898238346126961 & 0.1019058820076616\end{array}$

$\begin{array}{llll}0.5532585329096608 & 0.0558475197300061 & 0.1030409998700241\end{array}$

$\begin{array}{llll}0.8863864251620549 & 0.5575356785603310 & 0.1038239590699997\end{array}$

$\begin{array}{llll}0.0535761390370923 & 0.5563090400234042 & 0.1049005272364926\end{array}$

$\begin{array}{llll}0.2190795584965791 & 0.3917049910488729 & 0.1035750458142774\end{array}$

$\begin{array}{llll}0.3866701011712401 & 0.7227091427466837 & 0.1026195685400605\end{array}$ 
$\begin{array}{llll}0.5530308517253617 & 0.7229329672690631 & 0.1026550857273924\end{array}$

$\begin{array}{llll}0.7193365202717881 & 0.7238065650964400 & 0.1026542132902981\end{array}$

$\begin{array}{llll}0.7180782724781588 & 0.5566062793988459 & 0.1033955553345799\end{array}$

$\begin{array}{llll}0.5521883773467587 & 0.5559109755182586 & 0.1038298896003899\end{array}$

$\begin{array}{llll}0.8866955910518509 & 0.3915264897560439 & 0.1028170160562154\end{array}$

$\begin{array}{llll}0.0523744890087485 & 0.3928481948526070 & 0.1032527774193139\end{array}$

$\begin{array}{llll}0.7203604380735099 & 0.3909291147688523 & 0.1041224915807025\end{array}$

$\begin{array}{llll}0.2188438179186945 & 0.5553344382076078 & 0.1035843633607105\end{array}$

$\begin{array}{llll}0.2205139178429888 & 0.0563372203772504 & 0.1037063923805012\end{array}$

$\begin{array}{llll}0.8857103146132206 & 0.0568024218390054 & 0.1037854290002576\end{array}$

$\begin{array}{llll}0.0544233069111731 & 0.0542814123834178 & 0.1029305200600179\end{array}$

$\begin{array}{llll}0.8852163513095666 & 0.2220271881370790 & 0.1027205539355151\end{array}$

$\begin{array}{llll}0.0543366771350564 & 0.2214651714343829 & 0.1028642264583573\end{array}$

$\begin{array}{llll}0.5560665520775318 & 0.3900472242668273 & 0.1036611780284415\end{array}$

$\begin{array}{llll}0.7191149770540423 & 0.0581543725865751 & 0.1042046746630302\end{array}$

$\begin{array}{llll}0.7187790398739252 & 0.2231208029759656 & 0.1046281371955378\end{array}$

$\begin{array}{llll}0.5550976544844529 & 0.2206241961812293 & 0.1033419722881838\end{array}$

$\begin{array}{llll}0.3863485357609201 & 0.2227538719874640 & 0.1026344256191789\end{array}$

$\begin{array}{llll}0.2204494401328278 & 0.7218255253977972 & 0.1028960639905278\end{array}$

$\begin{array}{llll}0.3873324900226524 & 0.0552887437858910 & 0.1027799864980714\end{array}$

$\begin{array}{llll}0.2189253070185906 & 0.2233146899873043 & 0.1033566926622117\end{array}$

$\begin{array}{llll}0.8858610307765024 & 0.7240990334972385 & 0.1030536581835760\end{array}$

$\begin{array}{llll}0.0527401561466941 & 0.7234275951615706 & 0.1030131349381834\end{array}$

$\begin{array}{llll}0.3261725026814360 & 0.5020633397207062 & 0.1530560459096691\end{array}$

$\begin{array}{lllll}0.4943178206878401 & 0.1646566067411441 & 0.1539232809845882\end{array}$

$\begin{array}{llll}0.9909230675737791 & 0.5071675934181007 & 0.1566907499906619\end{array}$

$\begin{array}{llll}0.1562984476687440 & 0.5054312162782378 & 0.1580650419682212\end{array}$

$\begin{array}{llll}0.3283625577485199 & 0.6694526221932571 & 0.1537180143883906\end{array}$

$\begin{array}{llll}0.3308508592630185 & 0.3323397121968262 & 0.1521833279376812\end{array}$

$\begin{array}{llll}0.0008215503493357 & 0.3328579566853117 & 0.1517560663544772\end{array}$

$\begin{array}{llll}0.1637756884925984 & 0.3348978210903586 & 0.1530263124230085\end{array}$

$\begin{array}{llll}0.4941509728257616 & 0.5020548408533064 & 0.1571800872748614\end{array}$

$\begin{array}{llll}0.4933155388838153 & 0.6723639943550668 & 0.1537051250393462\end{array}$

$\begin{array}{llll}0.6616171495456903 & 0.8368791974452870 & 0.1543126591886995\end{array}$

$\begin{array}{llll}0.4963456563362595 & 0.3336257762736936 & 0.1507195911529347\end{array}$

$\begin{array}{llll}0.6677248705152403 & 0.3333365528167597 & 0.1553033322293153\end{array}$

$\begin{array}{llll}0.8243548781164310 & 0.5066214476949432 & 0.1535955226578804\end{array}$

$\begin{array}{llll}0.4954966867928204 & 0.8357886744574385 & 0.1544103991373659\end{array}$

$\begin{array}{llll}0.6559627761731271 & 0.5081744465419322 & 0.1553339358658276\end{array}$

$\begin{array}{llll}0.6603879741869088 & 0.6735660770854550 & 0.1535737636316170\end{array}$

$\begin{array}{llll}0.8258070936462883 & 0.6736966295951742 & 0.1539846377964815\end{array}$

$\begin{array}{llll}0.8381253811333577 & 0.3311264504414315 & 0.1519994436767109\end{array}$

$\begin{array}{llll}0.9982354218808600 & 0.1619587924838950 & 0.1527484823398503\end{array}$

$\begin{array}{llll}0.1632610864475997 & 0.1654764974807666 & 0.1559823900045553\end{array}$

$\begin{array}{llll}0.8311713822433233 & 0.1631363542480885 & 0.1555751698409832\end{array}$

$\begin{array}{llll}0.8284298422758838 & 0.8366176560482500 & 0.1546832513317307\end{array}$

$\begin{array}{llll}0.9955499122272196 & 0.8354356212539213 & 0.1545422956436869\end{array}$ 
0.66113979796553530 .16682060775444920 .1584028496292303 $\begin{array}{llll}0.3288413174333245 & 0.1654302066886662 & 0.1534359278967753\end{array}$ $\begin{array}{llll}0.9924667947229963 & 0.6731780099968360 & 0.1544043148666953\end{array}$ $0.16081164580222190 .6701863703145052 \quad 0.1537504771815763$ $\begin{array}{llll}0.8333399999999997 & 0.8333399999999997 & 0.0000000000000000\end{array}$ $\begin{array}{llll}0.0000100000000032 & 0.8333399999999997 & 0.0000000000000000\end{array}$ $\begin{array}{llll}0.6666700000000034 & 0.8333399999999997 & 0.0000000000000000\end{array}$ $\begin{array}{llll}0.3333299999999966 & 0.8333399999999997 & 0.0000000000000000\end{array}$ $\begin{array}{llll}0.5000000000000000 & 0.8333399999999997 & 0.0000000000000000\end{array}$ $\begin{array}{lll}0.0000000000000000 & 0.8333399999999997 & 0.0000000000000000\end{array}$ $\begin{array}{lll}0.1666700000000034 & 0.8333399999999997 & 0.0000000000000000\end{array}$ $\begin{array}{llll}0.9444500000000033 & 0.9444500000000033 & 0.0521799999999999\end{array}$ $\begin{array}{llll}0.7777799999999999 & 0.9444500000000033 & 0.0521799999999999\end{array}$ $\begin{array}{lll}0.4444500000000033 & 0.9444500000000033 & 0.0521799999999999\end{array}$ 0.61110999999999650 .94445000000000330 .0521799999999999 $\begin{array}{llll}0.1111099999999965 & 0.9444500000000033 & 0.0521799999999999\end{array}$ 0.27777999999999990 .94445000000000330 .0521799999999999 $\begin{array}{llll}0.5534698603355417 & 0.8892280928162298 & 0.1029084798298173\end{array}$ $0.2211202716667784 \quad 0.8884622405485514 \quad 0.1030521393220275$ $\begin{array}{llll}0.8857200263365147 & 0.8905026463199696 & 0.1032900104283948\end{array}$ $\begin{array}{llll}0.0534971596312009 & 0.8888510186714361 & 0.1031264485732450\end{array}$ $\begin{array}{llll}0.7196602885453620 & 0.8901914576600002 & 0.1029657316188133\end{array}$ $\begin{array}{llll}0.3872409893091545 & 0.8891786756763420 & 0.1029676646360644\end{array}$ $\begin{array}{llll}0.4959684253053154 & 0.0002220804317134 & 0.1543486023386791\end{array}$ $\begin{array}{llll}0.3293857720271886 & 0.8349378514375876 & 0.1543017898889653\end{array}$ $\begin{array}{llll}0.1629075973453893 & 0.8340958011145290 & 0.1541417919113631\end{array}$ $\begin{array}{llll}0.9971606248564759 & 0.9987471236119915 & 0.1545629163884517\end{array}$ $\begin{array}{llll}0.1637779077617887 & 0.9990655317978725 & 0.1541570896770413\end{array}$ $\begin{array}{llll}0.8296069868348234 & 0.0001576479248271 & 0.1550652493300607\end{array}$ $\begin{array}{llll}0.6623247418962311 & 0.0006428434620570 & 0.1542590665636291\end{array}$ $\begin{array}{llll}0.3300947481647590 & 0.0004753535125708 & 0.1545409980011202\end{array}$ $\begin{array}{llll}0.7516762662584372 & 0.2912242861056925 & 0.2028557717106968\end{array}$ $\begin{array}{llll}0.5102280020612187 & 0.4328254948382009 & 0.2041197166847166\end{array}$ $\begin{array}{llll}0.0734418374752603 & 0.2797458258729310 & 0.2045661537268315\end{array}$ $\begin{array}{llll}0.1905892474227445 & 0.4419003446400432 & 0.2059532747011196\end{array}$ $\begin{array}{llll}0.6524831044466498 & 0.4988384144902615 & 0.2088708077683649\end{array}$ $\begin{array}{llll}0.9785921335913383 & 0.4877096527180080 & 0.2097301519765004\end{array}$ $\begin{array}{llll}0.6146274917852389 & 0.2185713489252010 & 0.2106689425480233\end{array}$ $\begin{array}{llll}0.2866902758768331 & 0.2389191914917251 & 0.2238838159585901\end{array}$ $\begin{array}{llll}0.7583163560468522 & 0.4432117531353008 & 0.2117848865806918\end{array}$ $\begin{array}{llll}0.8156575522043236 & 0.4920658705665610 & 0.2141423947433158\end{array}$ $\begin{array}{llll}0.8062555071638814 & 0.3364583070005526 & 0.2069802044099838\end{array}$ $\begin{array}{llll}0.9216465490993501 & 0.4378681669830476 & 0.2121144452180180\end{array}$ $\begin{array}{llll}0.9140706051889539 & 0.2821516607532225 & 0.2058230970442606\end{array}$ $\begin{array}{llll}0.9742708094230438 & 0.3304906585634556 & 0.2077606716567569\end{array}$ $\begin{array}{llll}0.4586061778051924 & 0.3845015268983772 & 0.2076167693224057\end{array}$ $\begin{array}{llll}0.5072320324589532 & 0.2779371503099927 & 0.2126000244371055\end{array}$ 
$\begin{array}{llll}0.3502631365641504 & 0.4378127339802124 & 0.2056745567392634\end{array}$

$\begin{array}{llll}0.4492056964737914 & 0.2306478392766478 & 0.2170084321675193\end{array}$

$\begin{array}{llll}0.2902942863589976 & 0.3904447195596299 & 0.2088287914103357\end{array}$

$\begin{array}{llll}0.3424229208831074 & 0.2838637448523648 & 0.2162006861393004\end{array}$

$\begin{array}{llll}0.6187617074170816 & 0.5689438607525824 & 0.2190770478632182\end{array}$

$\begin{array}{llll}0.6155488165866646 & 0.4599966604136184 & 0.2147215382257822\end{array}$

$\begin{array}{llll}0.9449274849573644 & 0.5568599280850306 & 0.2207122734085286\end{array}$

$\begin{array}{llll}0.7766079080781194 & 0.5732355051314865 & 0.2185497493042538\end{array}$

$\begin{array}{llll}0.9525862222597512 & 0.1999649342323701 & 0.2080890297486082\end{array}$

$\begin{array}{llll}0.0522859096335178 & 0.4451181188255576 & 0.2173442421938612\end{array}$

$\begin{array}{llll}0.6500316353077530 & 0.2592496787089015 & 0.2157684224372196\end{array}$

$\begin{array}{llll}0.4876969789526058 & 0.1502437995021415 & 0.2228260132705182\end{array}$

$\begin{array}{llll}0.3109146892626886 & 0.5203440856071961 & 0.2078026452851946\end{array}$

$\begin{array}{llll}0.6431325289636959 & 0.1558630316367809 & 0.2253148870248119\end{array}$

$\begin{array}{llll}0.2115129951957949 & 0.2767054953100452 & 0.2185322399499381\end{array}$

$\begin{array}{llll}0.3202545713244188 & 0.1626733854081207 & 0.2223037903046627\end{array}$

Zwitterion Hydrogen Bond Network Higher Coverage - VASP- DFT-D2

$\mathrm{Cu} \mathrm{O}$ N C H

1.00000000000000

$\begin{array}{llll}15.3369999999999997 & 0.0000000000000000 & 0.0000000000000000\end{array}$

$\begin{array}{llll}5.1124999999999998 & 8.8551099999999998 & 0.0000000000000000\end{array}$

$\begin{array}{llll}0.0000000000000000 & 0.0000000000000000 & 40.0000000000000000\end{array}$

$\begin{array}{lllll}100 & 4 & 4 & 12 & 12\end{array}$

Direct

$\begin{array}{llll}0.0000100000000032 & 0.7499800000000008 & 0.0000000000000000\end{array}$

$\begin{array}{llll}0.8333399999999997 & 0.4999800000000008 & 0.0000000000000000\end{array}$

$\begin{array}{lll}0.8333399999999997 & 0.7499800000000008 & 0.0000000000000000\end{array}$

$\begin{array}{lll}0.0000100000000032 & 0.4999800000000008 & 0.0000000000000000\end{array}$

$\begin{array}{lll}0.0000100000000032 & 0.2500000000000000 & 0.0000000000000000\end{array}$

$\begin{array}{llll}0.8333399999999997 & 0.0000000000000000 & 0.0000000000000000\end{array}$

$\begin{array}{llll}0.8333399999999997 & 0.2500000000000000 & 0.0000000000000000\end{array}$

$\begin{array}{lll}0.0000100000000032 & 0.0000000000000000 & 0.0000000000000000\end{array}$

0.66667000000000340 .49998000000000080 .0000000000000000

$\begin{array}{lll}0.6666700000000034 & 0.7499800000000008 & 0.0000000000000000\end{array}$

$\begin{array}{llll}0.5000000000000000 & 0.7499800000000008 & 0.0000000000000000\end{array}$

$\begin{array}{llll}0.3333299999999966 & 0.4999800000000008 & 0.0000000000000000\end{array}$

$\begin{array}{lll}0.3333299999999966 & 0.7499800000000008 & 0.0000000000000000\end{array}$

0.16667000000000340 .74998000000000080 .0000000000000000

$\begin{array}{llll}0.6666700000000034 & 0.0000000000000000 & 0.0000000000000000\end{array}$

$\begin{array}{lll}0.5000000000000000 & 0.4999800000000008 & 0.0000000000000000\end{array}$

0.66667000000000340 .25000000000000000 .0000000000000000

$\begin{array}{lll}0.5000000000000000 & 0.2500000000000000 & 0.0000000000000000\end{array}$

$\begin{array}{lll}0.0000000000000000 & 0.4999800000000008 & 0.0000000000000000\end{array}$

$\begin{array}{llll}0.0000000000000000 & 0.7499800000000008 & 0.0000000000000000\end{array}$

$\begin{array}{lll}0.3333299999999966 & 0.0000000000000000 & 0.0000000000000000\end{array}$

$\begin{array}{lll}0.1666700000000034 & 0.4999800000000008 & 0.0000000000000000\end{array}$ 
$\begin{array}{lll}0.3333299999999966 & 0.2500000000000000 & 0.0000000000000000\end{array}$

$\begin{array}{lll}0.1666700000000034 & 0.2500000000000000 & 0.0000000000000000\end{array}$

$\begin{array}{llll}0.5000000000000000 & 0.0000000000000000 & 0.0000000000000000\end{array}$

$0.0000000000000000 \quad 0.0000000000000000 \quad 0.0000000000000000$

$\begin{array}{lll}0.0000000000000000 & 0.2500000000000000 & 0.0000000000000000\end{array}$

$\begin{array}{llll}0.1666700000000034 & 0.0000000000000000 & 0.0000000000000000\end{array}$

$\begin{array}{llll}0.9444500000000033 & 0.6666499999999971 & 0.0521799999999999\end{array}$

$\begin{array}{llll}0.9444500000000033 & 0.9166299999999978 & 0.0521799999999999\end{array}$

0.94445000000000330 .16666000000000030 .0521799999999999

0.94445000000000330 .41666000000000030 .0521799999999999

$\begin{array}{llll}0.7777799999999999 & 0.6666499999999971 & 0.0521799999999999\end{array}$

$\begin{array}{llll}0.7777799999999999 & 0.9166299999999978 & 0.0521799999999999\end{array}$

$\begin{array}{llll}0.6111099999999965 & 0.9166299999999978 & 0.0521799999999999\end{array}$

$\begin{array}{llll}0.4444500000000033 & 0.6666499999999971 & 0.0521799999999999\end{array}$

$\begin{array}{llll}0.4444500000000033 & 0.9166299999999978 & 0.0521799999999999\end{array}$

$\begin{array}{llll}0.2777799999999999 & 0.9166299999999978 & 0.0521799999999999\end{array}$

$\begin{array}{llll}0.7777799999999999 & 0.1666600000000003 & 0.0521799999999999\end{array}$

$\begin{array}{llll}0.6111099999999965 & 0.6666499999999971 & 0.0521799999999999\end{array}$

$\begin{array}{llll}0.7777799999999999 & 0.4166600000000003 & 0.0521799999999999\end{array}$

$\begin{array}{llll}0.6111099999999965 & 0.4166600000000003 & 0.0521799999999999\end{array}$

$\begin{array}{llll}0.1111099999999965 & 0.6666499999999971 & 0.0521799999999999\end{array}$

$\begin{array}{llll}0.1111099999999965 & 0.9166299999999978 & 0.0521799999999999\end{array}$

0.44445000000000330 .16666000000000030 .0521799999999999

$\begin{array}{llll}0.2780399999999972 & 0.6608900000000020 & 0.0521799999999999\end{array}$

$\begin{array}{llll}0.4444500000000033 & 0.4166600000000003 & 0.0521799999999999\end{array}$

$\begin{array}{llll}0.2777799999999999 & 0.4166600000000003 & 0.0521799999999999\end{array}$

$\begin{array}{llll}0.6111099999999965 & 0.1666600000000003 & 0.0521799999999999\end{array}$

$\begin{array}{llll}0.1111099999999965 & 0.1666600000000003 & 0.0521799999999999\end{array}$

$\begin{array}{lll}0.1111099999999965 & 0.4166600000000003 & 0.0521799999999999\end{array}$

$\begin{array}{llll}0.2777799999999999 & 0.1666600000000003 & 0.0521799999999999\end{array}$

$\begin{array}{llll}0.3869201848647723 & 0.8348729418228550 & 0.1088999432523924\end{array}$

$\begin{array}{llll}0.3848240566533402 & 0.5867182409929069 & 0.1054861294382752\end{array}$

$\begin{array}{llll}0.5523316415141103 & 0.0830562071454462 & 0.1070063312750284\end{array}$

$\begin{array}{llll}0.8885096560278997 & 0.8358597232989814 & 0.1084487868326170\end{array}$

$\begin{array}{llll}0.0554043941433519 & 0.8294414340178536 & 0.1195337160836189\end{array}$

$\begin{array}{llll}0.2172987257055102 & 0.5866078664236160 & 0.1066816347564447\end{array}$

$\begin{array}{llll}0.7178597948602621 & 0.8350608882820113 & 0.1077132391782595\end{array}$

$\begin{array}{llll}0.5527989702043826 & 0.8329431513697287 & 0.1112231800934721\end{array}$

$\begin{array}{llll}0.8856918968279139 & 0.5860086081754288 & 0.1059641411855624\end{array}$

$\begin{array}{llll}0.0519022982430699 & 0.5877316826160867 & 0.1072162664920395\end{array}$

$\begin{array}{llll}0.7181075357914218 & 0.5867471388014226 & 0.1068471728160431\end{array}$

$\begin{array}{llll}0.2170790104371553 & 0.8348326605032363 & 0.1077899909443441\end{array}$

$\begin{array}{llll}0.2190484046579519 & 0.0835605411794338 & 0.1082190959873927\end{array}$

$\begin{array}{llll}0.8878199833094320 & 0.0832227361957018 & 0.1085702471760582\end{array}$

$\begin{array}{llll}0.0524408248962971 & 0.0814335944024097 & 0.1085085223264997\end{array}$

$\begin{array}{llll}0.8865711217932837 & 0.3319544338625612 & 0.1067784590189319\end{array}$

$\begin{array}{llll}0.0542387914773925 & 0.3310884796623652 & 0.1076003591230352\end{array}$ 
$\begin{array}{lll}0.5528415162892876 & 0.5861402199193985 & 0.1063891938704020\end{array}$

$\begin{array}{llll}0.7206310908197230 & 0.0833061809945078 & 0.1072162129375149\end{array}$

$\begin{array}{llll}0.7215101158805723 & 0.3323157286439281 & 0.1074599621526894\end{array}$

$\begin{array}{llll}0.5541341819798482 & 0.3318450373650275 & 0.1063498565294936\end{array}$

$\begin{array}{llll}0.3856803308550090 & 0.3329101537381035 & 0.1066114474520947\end{array}$

$\begin{array}{lllll}0.3856070686088796 & 0.0836790623761645 & 0.1073873667871238\end{array}$

$\begin{array}{llll}0.2182300609217213 & 0.3336525088189662 & 0.1076938882530907\end{array}$

$\begin{array}{llll}0.3232990368462071 & 0.7576459808435673 & 0.1610419079477671\end{array}$

$\begin{array}{llll}0.4929633333351329 & 0.2535149512905122 & 0.1619631725232297\end{array}$

$\begin{array}{llll}0.9910765955719614 & 0.7579116481328226 & 0.1734641189938373\end{array}$

$\begin{array}{llll}0.1582267465504656 & 0.7590057241261031 & 0.1770013452018943\end{array}$

$\begin{array}{llll}0.3206786968565632 & 0.0134754874478152 & 0.1645469146893822\end{array}$

$\begin{array}{llll}0.3264707217123737 & 0.5052139114927873 & 0.1587474529942889\end{array}$

$\begin{array}{llll}0.9954858343286420 & 0.5029921646177479 & 0.1586068134191014\end{array}$

$\begin{array}{lllll}0.1591836668435711 & 0.5080423472824106 & 0.1605197752598088\end{array}$

$\begin{array}{llll}0.4903543223949168 & 0.7546533304873705 & 0.1701413237006279\end{array}$

$\begin{array}{llll}0.4907401230507366 & 0.0068872863169128 & 0.1640933742619725\end{array}$

$\begin{array}{llll}0.4936510658020048 & 0.5037072549367139 & 0.1578316915199818\end{array}$

$\begin{array}{llll}0.6614947469590596 & 0.5024980133631637 & 0.1597462901691655\end{array}$

$\begin{array}{llll}0.8269611349499846 & 0.7568876452866697 & 0.1605759479494493\end{array}$

$\begin{array}{llll}0.6587616909525381 & 0.7544887775841974 & 0.1690612282004913\end{array}$

$\begin{array}{llll}0.6598050409104419 & 0.0059273303581423 & 0.1622823500478273\end{array}$

$\begin{array}{llll}0.8266204426853506 & 0.0123189766259439 & 0.1638988705360930\end{array}$

$\begin{array}{llll}0.8288728832075942 & 0.5017943600534157 & 0.1583363833837295\end{array}$

$\begin{array}{llll}0.9933456449319796 & 0.2559707265652481 & 0.1619546394102335\end{array}$

$\begin{array}{llll}0.1573519498419977 & 0.2624974818994644 & 0.1856057666380226\end{array}$

$\begin{array}{llll}0.8224120202404316 & 0.2604583231112638 & 0.1835794612905484\end{array}$

$\begin{array}{llll}0.6618201255467682 & 0.2545663007153213 & 0.1623300740621354\end{array}$

$\begin{array}{llll}0.3224386499554374 & 0.2565850179793594 & 0.1628834216576094\end{array}$

$\begin{array}{llll}0.9882214870453206 & 0.0167573098962019 & 0.1680151284020200\end{array}$

$\begin{array}{llll}0.1576198762710349 & 0.0119910003447999 & 0.1642865099028723\end{array}$

$\begin{array}{llll}0.7832151229471681 & 0.3961268027433889 & 0.2260353193530714\end{array}$

$\begin{array}{llll}0.5104185053885795 & 0.6534783635346161 & 0.2256166493184154\end{array}$

$\begin{array}{llll}0.0968460342980483 & 0.3970326832420493 & 0.2281821168506287\end{array}$

$\begin{array}{llll}0.1979657227069375 & 0.6614449844145942 & 0.2275569500790786\end{array}$

$\begin{array}{llll}0.6762767393653708 & 0.7133836855941454 & 0.2265699119012923\end{array}$

$\begin{array}{llll}0.9916786583413844 & 0.7146459894996450 & 0.2295530867934369\end{array}$

$\begin{array}{llll}0.6116426876194182 & 0.3507684065783878 & 0.2399243081875839\end{array}$

$\begin{array}{llll}0.2945881946959489 & 0.3595174569221246 & 0.2440705893536698\end{array}$

$\begin{array}{llll}0.7812725721448383 & 0.6314750477185109 & 0.2339609078236334\end{array}$

$\begin{array}{lllll}0.8354431713792124 & 0.7081102603010541 & 0.2367070084857579\end{array}$

$\begin{array}{llll}0.8342241484285926 & 0.4696485927927465 & 0.2317406308747150\end{array}$

$\begin{array}{llll}0.9409375415061650 & 0.6316949878918872 & 0.2354377309369375\end{array}$

$\begin{array}{llll}0.9406954923525499 & 0.3937850588276545 & 0.2332279376387139\end{array}$

$\begin{array}{llll}0.9964783166786813 & 0.4699403350551633 & 0.2329922442237980\end{array}$

$\begin{array}{lllll}0.4572491582733733 & 0.5911172104312190 & 0.2332990286564666\end{array}$

$\begin{array}{llll}0.5100167904859060 & 0.4255653099760311 & 0.2403142236704182\end{array}$ 
0.35088144015724440 .66849142110888460 .2323097207637697

$\begin{array}{llll}0.4543443144793369 & 0.3519582259112914 & 0.2446313834346286\end{array}$

$\begin{array}{llll}0.2940721511910914 & 0.5950017292512918 & 0.2344232738539886\end{array}$

$\begin{array}{lll}0.3488994028335062 & 0.4300060975854999 & 0.2418989093352722\end{array}$

$\begin{array}{llll}0.6396322892084361 & 0.8187937664834104 & 0.2365638957360295\end{array}$

$\begin{array}{llll}0.6377799880312945 & 0.6580565980166148 & 0.2317205184499098\end{array}$

$\begin{array}{llll}0.9566567504234158 & 0.8195081941243315 & 0.2398847190099630\end{array}$

$\begin{array}{llll}0.7944005933290242 & 0.8309414873174712 & 0.2369317402621350\end{array}$

$\begin{array}{llll}0.9815420027513885 & 0.2714764291947573 & 0.2324995006423342\end{array}$

$\begin{array}{llll}0.0663649490650356 & 0.6593939549280682 & 0.2356144370328550\end{array}$

$\begin{array}{llll}0.6523316575519614 & 0.4037077627211195 & 0.2361980209756133\end{array}$

$\begin{array}{llll}0.4937759285562384 & 0.2306020809246694 & 0.2485387739208582\end{array}$

$\begin{array}{llll}0.3113494287978907 & 0.7891962697248704 & 0.2279424122606777\end{array}$

$\begin{array}{llll}0.6509461520714798 & 0.2371944444678529 & 0.2422217106469164\end{array}$

$\begin{array}{llll}0.2187444498465124 & 0.4133760816256261 & 0.2400507339304320\end{array}$

$\begin{array}{lll}0.3295108497695439 & 0.2460945429733080 & 0.2466824553135794\end{array}$

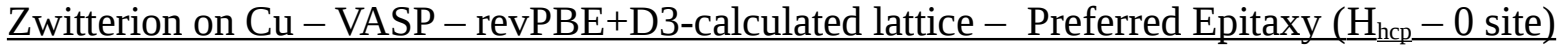

$\mathrm{Cu} \mathrm{O} \mathrm{N} \mathrm{C} \mathrm{H}$

1.00000000000000

$\begin{array}{lll}12.5239999999999991 & 0.0000000000000000 & 0.0000000000000000\end{array}$

$\begin{array}{lll}0.0000000000000000 & 13.0160000000000000 & 0.0000000000000000\end{array}$

$\begin{array}{llll}0.0000000000000000 & 0.0000000000000000 & 40.0000000000000000\end{array}$

$\begin{array}{llllll}\mathrm{Cu} & \mathrm{O} & \mathrm{N} & \mathrm{C} & \mathrm{H}\end{array}$

$\begin{array}{lllll}120 & 2 & 2 & 6 & 6\end{array}$

Direct

$0.0000000000000000 \quad 0.66664105716041890 .0000000000000000$

$\begin{array}{lll}0.2000159693388710 & 0.6666410571604189 & 0.0000000000000000\end{array}$

$\begin{array}{llll}0.3999520919833941 & 0.6666410571604189 & 0.0000000000000000\end{array}$

$\begin{array}{llll}0.2999840306611290 & 0.8332821143208378 & 0.0000000000000000\end{array}$

$\begin{array}{lllll}0.0000000000000000 & 0.3332821143208378 & 0.0000000000000000\end{array}$

$\begin{array}{llll}0.2000159693388710 & 0.3332821143208378 & 0.0000000000000000\end{array}$

$\begin{array}{llll}0.0999680613222580 & 0.4999231714812566 & 0.0000000000000000\end{array}$

$\begin{array}{llll}0.0999680613222580 & 0.8332821143208378 & 0.0000000000000000\end{array}$

$\begin{array}{lllll}0.0000000000000000 & 0.0000000000000000 & 0.0000000000000000\end{array}$

$\begin{array}{llll}0.5999680613222580 & 0.6666410571604189 & 0.0000000000000000\end{array}$

$\begin{array}{llll}0.7999840306611290 & 0.6666410571604189 & 0.0000000000000000\end{array}$

$\begin{array}{llll}0.7000159693388710 & 0.8332821143208378 & 0.0000000000000000\end{array}$

$\begin{array}{llll}0.3999520919833941 & 0.3332821143208378 & 0.0000000000000000\end{array}$

$\begin{array}{llll}0.5999680613222580 & 0.3332821143208378 & 0.0000000000000000\end{array}$

$\begin{array}{llll}0.5000000000000000 & 0.4999231714812566 & 0.0000000000000000\end{array}$

$\begin{array}{llll}0.5000000000000000 & 0.8332821143208378 & 0.0000000000000000\end{array}$

$\begin{array}{llll}0.2000159693388710 & 0.0000000000000000 & 0.0000000000000000\end{array}$

$\begin{array}{lllll}0.3999520919833941 & 0.0000000000000000 & 0.0000000000000000\end{array}$

$\begin{array}{llll}0.2999840306611290 & 0.1666410571604189 & 0.0000000000000000\end{array}$

$\begin{array}{llll}0.2999840306611290 & 0.4999231714812566 & 0.0000000000000000\end{array}$

$\begin{array}{llll}0.0999680613222580 & 0.1666410571604189 & 0.0000000000000000\end{array}$ 
$\begin{array}{lll}0.7999840306611290 & 0.3332821143208378 & 0.0000000000000000\end{array}$

$\begin{array}{llll}0.8999520919833941 & 0.4999231714812566 & 0.0000000000000000\end{array}$

$\begin{array}{llll}0.8999520919833941 & 0.8332821143208378 & 0.0000000000000000\end{array}$

$\begin{array}{lll}0.5999680613222580 & 0.0000000000000000 & 0.0000000000000000\end{array}$

$\begin{array}{lll}0.7999840306611290 & 0.0000000000000000 & 0.0000000000000000\end{array}$

$\begin{array}{llll}0.7000159693388710 & 0.1666410571604189 & 0.0000000000000000\end{array}$

$\begin{array}{llll}0.7000159693388710 & 0.4999231714812566 & 0.0000000000000000\end{array}$

$\begin{array}{llll}0.5000000000000000 & 0.1666410571604189 & 0.0000000000000000\end{array}$

$\begin{array}{lll}0.8999520919833941 & 0.1666410571604189 & 0.0000000000000000\end{array}$

$\begin{array}{llll}0.0000000000000000 & 0.8888291333743084 & 0.0511249999999990\end{array}$

$\begin{array}{llll}0.2000159693388710 & 0.8888291333743084 & 0.0511249999999990\end{array}$

$\begin{array}{llll}0.3999520919833941 & 0.8888291333743084 & 0.0511249999999990\end{array}$

$\begin{array}{llll}0.0000000000000000 & 0.5554701905347272 & 0.0511249999999990\end{array}$

$\begin{array}{llll}0.2000159693388710 & 0.5554701905347272 & 0.0511249999999990\end{array}$

$\begin{array}{llll}0.0999680613222580 & 0.7221880762138895 & 0.0511249999999990\end{array}$

$\begin{array}{llll}0.0000000000000000 & 0.2221880762138895 & 0.0511249999999990\end{array}$

$\begin{array}{llll}0.5999680613222580 & 0.8888291333743084 & 0.0511249999999990\end{array}$

$\begin{array}{llll}0.7999840306611290 & 0.8888291333743084 & 0.0511249999999990\end{array}$

$\begin{array}{llll}0.3999520919833941 & 0.5554701905347272 & 0.0511249999999990\end{array}$

$\begin{array}{llll}0.5999680613222580 & 0.5554701905347272 & 0.0511249999999990\end{array}$

$\begin{array}{llll}0.5000000000000000 & 0.7221880762138895 & 0.0511249999999990\end{array}$

$\begin{array}{llll}0.2000159693388710 & 0.2221880762138895 & 0.0511249999999990\end{array}$

$\begin{array}{llll}0.3999520919833941 & 0.2221880762138895 & 0.0511249999999990\end{array}$

$\begin{array}{llll}0.2999840306611290 & 0.3888291333743084 & 0.0511249999999990\end{array}$

$\begin{array}{llll}0.2999840306611290 & 0.7221880762138895 & 0.0511249999999990\end{array}$

$\begin{array}{llll}0.0999680613222580 & 0.0555470190534706 & 0.0511249999999990\end{array}$

$\begin{array}{llll}0.0999680613222580 & 0.3888291333743084 & 0.0511249999999990\end{array}$

$\begin{array}{lll}0.7999840306611290 & 0.5554701905347272 & 0.0511249999999990\end{array}$

$\begin{array}{llll}0.8999520919833941 & 0.7221880762138895 & 0.0511249999999990\end{array}$

$\begin{array}{llll}0.5999680613222580 & 0.2221880762138895 & 0.0511249999999990\end{array}$

$\begin{array}{llll}0.7999840306611290 & 0.2221880762138895 & 0.0511249999999990\end{array}$

$\begin{array}{llll}0.7000159693388710 & 0.3888291333743084 & 0.0511249999999990\end{array}$

$\begin{array}{llll}0.7000159693388710 & 0.7221880762138895 & 0.0511249999999990\end{array}$

$\begin{array}{llll}0.5000000000000000 & 0.0555470190534706 & 0.0511249999999990\end{array}$

$\begin{array}{llll}0.5000000000000000 & 0.3888291333743084 & 0.0511249999999990\end{array}$

$\begin{array}{llll}0.2999840306611290 & 0.0555470190534706 & 0.0511249999999990\end{array}$

$\begin{array}{llll}0.8999520919833941 & 0.0555470190534706 & 0.0511249999999990\end{array}$

$\begin{array}{llll}0.8999520919833941 & 0.3888291333743084 & 0.0511249999999990\end{array}$

$\begin{array}{llll}0.7000159693388710 & 0.0555470190534706 & 0.0511249999999990\end{array}$

$\begin{array}{llll}0.4998455594259283 & 0.6106466200336058 & 0.1014019834553253\end{array}$

$\begin{array}{llll}0.3989163823691726 & 0.7788292289739260 & 0.1017853652160241\end{array}$

$\begin{array}{llll}0.6009343184024090 & 0.7786620611371902 & 0.1017540129546575\end{array}$

$\begin{array}{llll}0.7009770461466545 & 0.6104878735380268 & 0.1027933060481629\end{array}$

$\begin{array}{llll}0.2987009087286125 & 0.6104454942756448 & 0.1025195873441689\end{array}$

$\begin{array}{llll}0.1995920873068613 & 0.4444415464402240 & 0.1023826195382753\end{array}$

$\begin{array}{llll}0.8003440228672289 & 0.4444143549642519 & 0.1022626589743894\end{array}$

$\begin{array}{llll}0.3986533976651145 & 0.4442056301725543 & 0.1023054745634582\end{array}$ 
0.60110297334659890 .44414612827325330 .1022332637159451 $\begin{array}{llll}0.9004195916964761 & 0.2774832267819320 & 0.1021690462360212\end{array}$ $\begin{array}{llll}0.0996412386328984 & 0.2775389631165694 & 0.1021921556359260\end{array}$ $\begin{array}{lll}0.4998786537502440 & 0.9452126703407799 & 0.1020668795240312\end{array}$ $\begin{array}{llll}0.8000986072466318 & 0.1109414434272011 & 0.1022773542863717\end{array}$ 0.19986960606142650 .11093660177294140 .1022884224015357 $\begin{array}{llll}0.9999883770607951 & 0.1108543235279669 & 0.1022802072376905\end{array}$ $\begin{array}{llll}0.7002398332228097 & 0.9442905049274160 & 0.1025727375371446\end{array}$ $\begin{array}{llll}0.2996300309157007 & 0.9440067026678989 & 0.1027729441492298\end{array}$ $\begin{array}{llll}0.0999048108323350 & 0.9443822461150120 & 0.1022809390507575\end{array}$ $\begin{array}{llll}0.8999611401991032 & 0.9443319897729694 & 0.1022688989595508\end{array}$ $\begin{array}{llll}0.6000625441059242 & 0.1112631136515319 & 0.1022415624128712\end{array}$ $\begin{array}{llll}0.3997457140248173 & 0.1111993563667824 & 0.1022418140407382\end{array}$ $\begin{array}{llll}0.2993802422541094 & 0.2774934824035071 & 0.1021721444167625\end{array}$ $\begin{array}{llll}0.7005246381914247 & 0.2774186346930279 & 0.1021236925788500\end{array}$ $\begin{array}{llll}0.7998744120903893 & 0.7774445991873478 & 0.1029424116893765\end{array}$ $\begin{array}{llll}0.9998603558960539 & 0.4443801623819326 & 0.1020922736945664\end{array}$ $\begin{array}{llll}0.2000981924485643 & 0.7778924848420417 & 0.1028575466767450\end{array}$ $\begin{array}{llll}0.9998444402883493 & 0.7776606992424178 & 0.1022631130600047\end{array}$ $\begin{array}{llll}0.4998045618968305 & 0.2773549503678154 & 0.1018935026055061\end{array}$ $\begin{array}{llll}0.8992743128820706 & 0.6116826423627089 & 0.1026962565980796\end{array}$ $\begin{array}{llll}0.1000005236846278 & 0.6114924210884993 & 0.1024939646106731\end{array}$ 0.59839226994611750 .66801236355341590 .1514165550721245 0.40050925601725140 .66784002999877150 .1511972686027576 $\begin{array}{llll}0.2964956495105326 & 0.4976612637572941 & 0.1548982954295823\end{array}$ $\begin{array}{llll}0.7034660026221076 & 0.4967840513631145 & 0.1546177374922948\end{array}$ $\begin{array}{llll}0.4999732227866573 & 0.4975271388662748 & 0.1520376661550742\end{array}$ $\begin{array}{llll}0.5002415274866721 & 0.8355866718600282 & 0.1525248035548188\end{array}$ $\begin{array}{llll}0.0997098008769584 & 0.1665722282411082 & 0.1545324895030262\end{array}$ $\begin{array}{llll}0.9003728462684819 & 0.1665214968869222 & 0.1545313144199980\end{array}$ $\begin{array}{llll}0.1995229982062554 & 0.0003311404739321 & 0.1545319256876567\end{array}$ $\begin{array}{llll}0.8004443945664548 & 0.0004917085156393 & 0.1544888751976288\end{array}$ $\begin{array}{llll}0.0000035252791761 & 0.0004677290044697 & 0.1544671111436102\end{array}$ $\begin{array}{llll}0.0000032050031464 & 0.3332749279967118 & 0.1544630982295843\end{array}$ $\begin{array}{llll}0.7006203176049155 & 0.8349089215965364 & 0.1553330475076322\end{array}$ $\begin{array}{lllll}0.2995726631742244 & 0.8342869177960921 & 0.1561678142034850\end{array}$ $\begin{array}{llll}0.5999862055337104 & 0.0006325508399527 & 0.1543591531482065\end{array}$ $\begin{array}{llll}0.3999473692886113 & 0.0004985558899122 & 0.1543845092408773\end{array}$ $\begin{array}{llll}0.2997469225489908 & 0.1664332670890332 & 0.1545882346378775\end{array}$ $\begin{array}{llll}0.7001601997573772 & 0.1663645725253105 & 0.1545677440121267\end{array}$ $\begin{array}{llll}0.1991359001489030 & 0.3318771148383277 & 0.1542078906568136\end{array}$ $\begin{array}{llll}0.8009530461546106 & 0.3314449768305892 & 0.1540449119140419\end{array}$ $\begin{array}{llll}0.9009898419923275 & 0.8342194572124413 & 0.1544072466463575\end{array}$ $\begin{array}{llll}0.0989508263039980 & 0.8341362434304216 & 0.1543929679874836\end{array}$ $\begin{array}{llll}0.4998315064377010 & 0.1660123426709461 & 0.1546015276006614\end{array}$ 0.40001135210606980 .33083238368419640 .1536743122357507 $\begin{array}{llll}0.5996887085407820 & 0.3303591481469752 & 0.1534978456956466\end{array}$ 
$\begin{array}{lll}0.0982416254296226 & 0.4999136368037185 & 0.1542945894344285\end{array}$

$\begin{array}{llll}0.8003967998060944 & 0.6676879016256123 & 0.1562850249744514\end{array}$

$\begin{array}{llll}0.9014341014227227 & 0.4999301706412140 & 0.1542498022988639\end{array}$

0.19850726447234020 .66732586386111590 .1551554662251320

$\begin{array}{llll}0.9997274320290686 & 0.6669222191306048 & 0.1548000987368141\end{array}$

$\begin{array}{llll}0.3115997454130195 & 0.7167178176351783 & 0.2083224740730714\end{array}$

$\begin{array}{lllll}0.7002942954802549 & 0.7080715736021475 & 0.2096120466124560\end{array}$

$\begin{array}{llll}0.3038591366645420 & 0.5064435475269349 & 0.2090648945683995\end{array}$

$\begin{array}{lll}0.6981430908875623 & 0.4982163954348025 & 0.2088568264552606\end{array}$

$\begin{array}{llll}0.5063070166584538 & 0.7174620320433860 & 0.2106680529264224\end{array}$

$\begin{array}{lllll}0.4042703800397049 & 0.5560792403711901 & 0.2132009933197878\end{array}$

$\begin{array}{lllll}0.4033959313877844 & 0.6674117076916275 & 0.2111537776190744\end{array}$

$\begin{array}{llll}0.6000564240119317 & 0.5513945347980354 & 0.2131004042378066\end{array}$

$\begin{array}{llll}0.5008158590862521 & 0.5000209473080801 & 0.2143201979470817\end{array}$

$\begin{array}{llll}0.6065127269299495 & 0.6628423278003694 & 0.2115824793691985\end{array}$

$\begin{array}{llll}0.5087282473323640 & 0.8011972625327104 & 0.2126163224729751\end{array}$

$\begin{array}{llll}0.4987682829152078 & 0.4160840186851686 & 0.2155454530796348\end{array}$

$\begin{array}{llll}0.7618640037755426 & 0.5412970344597241 & 0.2164885235096321\end{array}$

$\begin{array}{llll}0.7005474161571925 & 0.4244930026478002 & 0.2172246845744041\end{array}$

$\begin{array}{llll}0.2986450702699983 & 0.4335496540614798 & 0.2181225580760042\end{array}$

$\begin{array}{llll}0.2419699199451699 & 0.5522477943718371 & 0.2164884235323044\end{array}$

Zwitterion on Ag - VASP - revPBE+D3-calculated lattice - Preferred Epitaxy ( $\mathrm{T}$ - 30 site)

Ag O N C H

1.00000000000000

$\begin{array}{lll}14.5060000000000002 & 0.0000000000000000 & 0.0000000000000000\end{array}$

$\begin{array}{lll}0.0000000000000000 & 15.0760000000000005 & 0.0000000000000000\end{array}$

$\begin{array}{lll}0.0000000000000000 & 0.0000000000000000 & 40.0000000000000000\end{array}$

Ag $\mathrm{O} \quad \mathrm{N} \quad \mathrm{C} \quad \mathrm{H}$

$\begin{array}{lllll}120 & 2 & 2 & 6 & 6\end{array}$

Direct

$0.8999710000000007 \quad 0.16665199999999910 .0000000000000000$

$\begin{array}{lll}0.4999839999999978 & 0.1666519999999991 & 0.0000000000000000\end{array}$

$\begin{array}{llll}0.6999769999999970 & 0.4999570000000020 & 0.0000000000000000\end{array}$

$\begin{array}{llll}0.6999769999999970 & 0.1666519999999991 & 0.0000000000000000\end{array}$

$0.79997399999999890 .0000000000000000 \quad 0.0000000000000000$

$\begin{array}{llll}0.5999809999999997 & 0.0000000000000000 & 0.0000000000000000\end{array}$

$\begin{array}{llll}0.8999710000000007 & 0.8332619999999977 & 0.0000000000000000\end{array}$

$\begin{array}{llll}0.8999710000000007 & 0.4999570000000020 & 0.0000000000000000\end{array}$

$\begin{array}{llll}0.7999739999999989 & 0.3333050000000028 & 0.0000000000000000\end{array}$

$\begin{array}{llll}0.0999970000000019 & 0.1666519999999991 & 0.0000000000000000\end{array}$

$\begin{array}{llll}0.2999909999999986 & 0.4999570000000020 & 0.0000000000000000\end{array}$

$\begin{array}{llll}0.2999909999999986 & 0.1666519999999991 & 0.0000000000000000\end{array}$

$\begin{array}{llll}0.3999870000000030 & 0.0000000000000000 & 0.0000000000000000\end{array}$

$\begin{array}{llll}0.1999939999999967 & 0.0000000000000000 & 0.0000000000000000\end{array}$

$\begin{array}{lllll}0.4999839999999978 & 0.8332619999999977 & 0.0000000000000000\end{array}$

$\begin{array}{llll}0.4999839999999978 & 0.4999570000000020 & 0.0000000000000000\end{array}$ 
$\begin{array}{lll}0.5999809999999997 & 0.3333050000000028 & 0.0000000000000000\end{array}$

$\begin{array}{llll}0.3999870000000030 & 0.3333050000000028 & 0.0000000000000000\end{array}$

$\begin{array}{llll}0.6999769999999970 & 0.8332619999999977 & 0.0000000000000000\end{array}$

$\begin{array}{lll}0.7999739999999989 & 0.6666090000000011 & 0.0000000000000000\end{array}$

$\begin{array}{llll}0.5999809999999997 & 0.6666090000000011 & 0.0000000000000000\end{array}$

$\begin{array}{llll}0.0000000000000000 & 0.0000000000000000 & 0.0000000000000000\end{array}$

$\begin{array}{llll}0.0999970000000019 & 0.8332619999999977 & 0.0000000000000000\end{array}$

$\begin{array}{llll}0.0999970000000019 & 0.4999570000000020 & 0.0000000000000000\end{array}$

$\begin{array}{llll}0.1999939999999967 & 0.3333050000000028 & 0.0000000000000000\end{array}$

$\begin{array}{llll}0.0000000000000000 & 0.3333050000000028 & 0.0000000000000000\end{array}$

$\begin{array}{llll}0.2999909999999986 & 0.8332619999999977 & 0.0000000000000000\end{array}$

$\begin{array}{llll}0.3999870000000030 & 0.6666090000000011 & 0.0000000000000000\end{array}$

$\begin{array}{llll}0.1999939999999967 & 0.6666090000000011 & 0.0000000000000000\end{array}$

$\begin{array}{llll}0.0000000000000000 & 0.6666090000000011 & 0.0000000000000000\end{array}$

$\begin{array}{llll}0.6999769999999970 & 0.0555510000000012 & 0.0592070000000007\end{array}$

$\begin{array}{llll}0.8999710000000007 & 0.3888549999999995 & 0.0592070000000007\end{array}$

$\begin{array}{llll}0.8999710000000007 & 0.0555510000000012 & 0.0592070000000007\end{array}$

$\begin{array}{llll}0.2999909999999986 & 0.0555510000000012 & 0.0592070000000007\end{array}$

$\begin{array}{llll}0.4999839999999978 & 0.3888549999999995 & 0.0592070000000007\end{array}$

$\begin{array}{llll}0.4999839999999978 & 0.0555510000000012 & 0.0592070000000007\end{array}$

$\begin{array}{llll}0.6999769999999970 & 0.7221600000000024 & 0.0592070000000007\end{array}$

$\begin{array}{llll}0.6999769999999970 & 0.3888549999999995 & 0.0592070000000007\end{array}$

$\begin{array}{lll}0.7999739999999989 & 0.2222030000000004 & 0.0592070000000007\end{array}$

$\begin{array}{llll}0.5999809999999997 & 0.2222030000000004 & 0.0592070000000007\end{array}$

$\begin{array}{llll}0.8999710000000007 & 0.7221600000000024 & 0.0592070000000007\end{array}$

$\begin{array}{llll}0.7999739999999989 & 0.5555080000000032 & 0.0592070000000007\end{array}$

$\begin{array}{lll}0.0999970000000019 & 0.3888549999999995 & 0.0592070000000007\end{array}$

$\begin{array}{lll}0.0999970000000019 & 0.0555510000000012 & 0.0592070000000007\end{array}$

$\begin{array}{llll}0.2999909999999986 & 0.7221600000000024 & 0.0592070000000007\end{array}$

$\begin{array}{llll}0.2999909999999986 & 0.3888549999999995 & 0.0592070000000007\end{array}$

$\begin{array}{llll}0.3999870000000030 & 0.2222030000000004 & 0.0592070000000007\end{array}$

$\begin{array}{llll}0.1999939999999967 & 0.2222030000000004 & 0.0592070000000007\end{array}$

$\begin{array}{lll}0.4999839999999978 & 0.7221600000000024 & 0.0592070000000007\end{array}$

$\begin{array}{llll}0.5999809999999997 & 0.5555080000000032 & 0.0592070000000007\end{array}$

$\begin{array}{llll}0.3999870000000030 & 0.5555080000000032 & 0.0592070000000007\end{array}$

$\begin{array}{llll}0.7999739999999989 & 0.8888120000000015 & 0.0592070000000007\end{array}$

$\begin{array}{llll}0.5999809999999997 & 0.8888120000000015 & 0.0592070000000007\end{array}$

$\begin{array}{llll}0.0000000000000000 & 0.2222030000000004 & 0.0592070000000007\end{array}$

0.09999700000000190 .72216000000000240 .0592070000000007

$\begin{array}{llll}0.1999939999999967 & 0.5555080000000032 & 0.0592070000000007\end{array}$

$\begin{array}{llll}0.0000000000000000 & 0.5555080000000032 & 0.0592070000000007\end{array}$

$\begin{array}{llll}0.3999870000000030 & 0.8888120000000015 & 0.0592070000000007\end{array}$

$\begin{array}{lll}0.1999939999999967 & 0.8888120000000015 & 0.0592070000000007\end{array}$

$\begin{array}{llll}0.0000000000000000 & 0.8888120000000015 & 0.0592070000000007\end{array}$

$\begin{array}{llll}0.6009880562456900 & 0.4418318365017271 & 0.1174680349350012\end{array}$

$\begin{array}{llll}0.5006255901420076 & 0.6103200072035051 & 0.1177810827299979\end{array}$

$\begin{array}{llll}0.3981139405418475 & 0.4414596072897297 & 0.1180042517150000\end{array}$ 
$\begin{array}{llll}0.5000471711085055 & 0.2753645674117777 & 0.1180402787124990\end{array}$ $\begin{array}{llll}0.2987639958017354 & 0.2741723086826724 & 0.1179873226350026\end{array}$ $\begin{array}{llll}0.8009766878601923 & 0.4410624119129736 & 0.1181132862350012\end{array}$ $\begin{array}{llll}0.7004883982765762 & 0.2749557991310709 & 0.1183497655675012\end{array}$ $\begin{array}{llll}0.7000059113263504 & 0.6092364982953029 & 0.1183503851050034\end{array}$ $\begin{array}{llll}0.3998977582103933 & 0.7757614376028101 & 0.1182586036400011\end{array}$ $\begin{array}{llll}0.6004019969116214 & 0.7758198658729114 & 0.1185606311575000\end{array}$ $\begin{array}{llll}0.1993260214669803 & 0.4407112739917736 & 0.1185377727699972\end{array}$ $\begin{array}{llll}0.2997989230318510 & 0.6069362375099487 & 0.1185401007574995\end{array}$ $\begin{array}{llll}0.5000169844202418 & 0.9424028084704190 & 0.1183676991250024\end{array}$ $\begin{array}{llll}0.6001386598786738 & 0.1089227988193144 & 0.1184156425374994\end{array}$ $\begin{array}{llll}0.3998967550944457 & 0.1087710103674695 & 0.1183957805899993\end{array}$ $0.7000772297256290 \quad 0.9422620049150936 \quad 0.1183796738850020$ $0.2998114000689398 \quad 0.94189478457150240 .1183761493275028$ 0.20005722555494290 .77427867590209590 .1183623329700012 $\begin{array}{lll}0.9000899637115651 & 0.9411750136641004 & 0.1184602742374992\end{array}$ $\begin{array}{llll}0.1996123671928842 & 0.1078241540726950 & 0.1184485079649988\end{array}$ $\begin{array}{llll}0.8003498032882916 & 0.7751233355001332 & 0.1184775193025018\end{array}$ $\begin{array}{llll}0.0999071184682165 & 0.9405349554855391 & 0.1183821187700005\end{array}$ $\begin{array}{llll}0.9000111439197553 & 0.2742542050676562 & 0.1185315646649983\end{array}$ $\begin{array}{llll}0.9000139442713362 & 0.6075316806447333 & 0.1185264429274966\end{array}$ $\begin{array}{llll}0.9998616946435988 & 0.4405802440700484 & 0.1185149129374992\end{array}$ $\begin{array}{llll}0.0993883589687030 & 0.2739752740713683 & 0.1184974609674967\end{array}$ $0.99986742972563290 .1075383226121005 \quad 0.1185273498125028$ $\begin{array}{llll}0.9999588356611042 & 0.7737990523083056 & 0.1185055299599966\end{array}$ $\begin{array}{llll}0.8001122527230109 & 0.1084974528190514 & 0.1186229460450008\end{array}$ $\begin{array}{llll}0.0998961855232352 & 0.6074811922326901 & 0.1185182774899971\end{array}$ 0.49981596870949830 .49721697605465920 .1743720532600008 $\begin{array}{llll}0.7020619042878806 & 0.4971592302799124 & 0.1766087264674994\end{array}$ $\begin{array}{llll}0.3982556209361618 & 0.3274731420137940 & 0.1768541712200005\end{array}$ $\begin{array}{llll}0.5996213063284159 & 0.3309222255173765 & 0.1780279988049998\end{array}$ $\begin{array}{llll}0.3997524690886536 & 0.6639598548089651 & 0.1771291834425028\end{array}$ $\begin{array}{llll}0.6030899068385480 & 0.6662439848301958 & 0.1786380125699978\end{array}$ $\begin{array}{llll}0.2956024656418066 & 0.4946803857256583 & 0.1790637165600018\end{array}$ $\begin{array}{llll}0.4999749477457627 & 0.1638298684929680 & 0.1790354969400028\end{array}$ $\begin{array}{llll}0.4999547099682857 & 0.8307653998806046 & 0.1788283692100023\end{array}$ $\begin{array}{llll}0.1979002267268726 & 0.3284219706354463 & 0.1790650173700001\end{array}$ $0.8015233591134674 \quad 0.6644514109843485 \quad 0.1791708164199974$ $\begin{array}{llll}0.7013945121260150 & 0.8321969293977176 & 0.1789291257424992\end{array}$ $\begin{array}{llll}0.2999868533365486 & 0.8304997834571495 & 0.1788663553524970\end{array}$ $0.2991606054253424 \quad 0.16295972417749740 .1791646008724967$ $\begin{array}{llll}0.6000618251757928 & 0.9974396226916937 & 0.1788447727950029\end{array}$ 0.39996883692954550 .99721744131732490 .1788748426500035 $\begin{array}{llll}0.7996550617261846 & 0.3306634602746072 & 0.1795369043774997\end{array}$ $\begin{array}{llll}0.1997527759685624 & 0.9970625385778717 & 0.1789004383049999\end{array}$ $\begin{array}{llll}0.9999580205225413 & 0.9972173860573079 & 0.1789981280300026\end{array}$ $0.1996886845925800 \quad 0.6638310807375944 \quad 0.1789051851050019$ 
$\begin{array}{llll}0.0979828363366906 & 0.4966796519434880 & 0.1790294650449979\end{array}$

$\begin{array}{llll}0.8003173833930788 & 0.9982294454430871 & 0.1791767879425024\end{array}$

$\begin{array}{llll}0.1000860991796486 & 0.8304946620390012 & 0.1790105226300014\end{array}$

$\begin{array}{llll}0.9007220529229301 & 0.8310196921862598 & 0.1793955010450006\end{array}$

$\begin{array}{lll}0.6995596232386632 & 0.1648410004311458 & 0.1795184348450007\end{array}$

$\begin{array}{llll}0.8997316005583897 & 0.4972298168280744 & 0.1799417387049971\end{array}$

$\begin{array}{llll}0.0989827882393470 & 0.1633035135049070 & 0.1795654111675020\end{array}$

$\begin{array}{llll}0.8995850846890932 & 0.1641681148713161 & 0.1797020197549983\end{array}$

$\begin{array}{llll}0.0001295993313093 & 0.6639639926903698 & 0.1796603440425031\end{array}$

$\begin{array}{llll}0.9987974951813072 & 0.3302903746152808 & 0.1796653859925001\end{array}$

$\begin{array}{lllll}0.5986357515028260 & 0.6523089359909804 & 0.2420302526999976\end{array}$

$\begin{array}{llll}0.3129600197918094 & 0.4907532493234257 & 0.2422400926250035\end{array}$

$\begin{array}{llll}0.4029214350613515 & 0.3393885516052038 & 0.2500269842674996\end{array}$

$\begin{array}{lll}0.6914339839032095 & 0.5002231676373015 & 0.2500508520675027\end{array}$

$\begin{array}{llll}0.4512858050530824 & 0.5789991093061815 & 0.2458397787750002\end{array}$

$\begin{array}{llll}0.5493148805597698 & 0.5823632828270107 & 0.2467587438800010\end{array}$

$\begin{array}{llll}0.4004258752240446 & 0.4983599099164238 & 0.2468360900750000\end{array}$

$\begin{array}{llll}0.4541165065490134 & 0.4143247679225226 & 0.2512122578700016\end{array}$

$\begin{array}{llll}0.5984256742382428 & 0.4955427670801242 & 0.2511849878524970\end{array}$

$\begin{array}{llll}0.5504830665173017 & 0.4148342719885889 & 0.2531858707025023\end{array}$

$\begin{array}{llll}0.4132186186957085 & 0.6413598771557432 & 0.2442852563100004\end{array}$

$\begin{array}{llll}0.3331670111884719 & 0.3472056060095525 & 0.2489943186799977\end{array}$

$\begin{array}{llll}0.7202846463601276 & 0.5617033913770229 & 0.2488836866625022\end{array}$

$\begin{array}{llll}0.7321348964428509 & 0.4459182268506225 & 0.2521707580324986\end{array}$

$\begin{array}{lll}0.4303991905556330 & 0.2780548378217063 & 0.2526571287074972\end{array}$

$\begin{array}{llll}0.5883894647111561 & 0.3524169952905254 & 0.2552155738850033\end{array}$

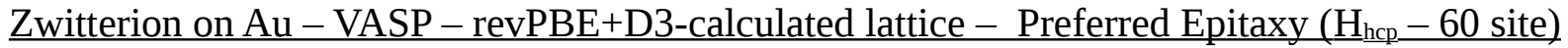

Au O N C H

1.00000000000000

$\begin{array}{llll}14.6319999999999997 & 0.0000000000000000 & 0.0000000000000000\end{array}$

$\begin{array}{lll}0.0000000000000000 & 15.2059999999999995 & 0.0000000000000000\end{array}$

$\begin{array}{lll}0.0000000000000000 & 0.0000000000000000 & 40.0000000000000000\end{array}$

$\mathrm{Au} \quad \mathrm{O} \quad \mathrm{N} \quad \mathrm{C} \quad \mathrm{H}$

$\begin{array}{lllll}120 & 2 & 2 & 6 & 6\end{array}$

Direct

$\begin{array}{lll}0.8981960877187021 & 0.5010223639813205 & 0.1849120829124971\end{array}$

$\begin{array}{llll}0.8034775154524354 & 0.6658722379060862 & 0.1843450637525024\end{array}$

$\begin{array}{llll}0.0009915891880823 & 0.6652871584440376 & 0.1843061444425018\end{array}$

$\begin{array}{llll}0.7986922618712384 & 0.3347278875115052 & 0.1840442353449987\end{array}$

$\begin{array}{llll}0.7003767188285934 & 0.4991605229646225 & 0.1838878481649999\end{array}$

$\begin{array}{llll}0.9019753804606339 & 0.8312197688675553 & 0.1833075481775026\end{array}$

$\begin{array}{llll}0.6012767942659920 & 0.3282988493029038 & 0.1832978777124978\end{array}$

$\begin{array}{llll}0.7023493905617855 & 0.1665185831185028 & 0.1830162672925013\end{array}$

$\begin{array}{llll}0.5007732564926215 & 0.1632100130014464 & 0.1828459801324982\end{array}$

0.80247355932203130 .99993800708930540 .1823782153550013

$\begin{array}{llll}0.2986269063490994 & 0.1631585359792211 & 0.1823130911649997\end{array}$ 
$\begin{array}{lll}0.0974575487629821 & 0.4996508828554482 & 0.1820432290975020\end{array}$

$\begin{array}{llll}0.7047636694983623 & 0.8339690914770514 & 0.1820343831849982\end{array}$

$\begin{array}{llll}0.6038129181793295 & 0.0018241896554017 & 0.1819729159524996\end{array}$

$\begin{array}{llll}0.1007002589871533 & 0.8299322025647768 & 0.1819308316900035\end{array}$

$\begin{array}{llll}0.2014690405002710 & 0.6637928980205174 & 0.1818221542449976\end{array}$

$\begin{array}{llll}0.1970970964461429 & 0.3276719130014456 & 0.1815237713975009\end{array}$

$\begin{array}{lllll}0.9973512688764359 & 0.3348192929369986 & 0.1814070793200031\end{array}$

$\begin{array}{llll}0.8993539481547259 & 0.1677877494607358 & 0.1812804015300031\end{array}$

$\begin{array}{llll}0.4058278697102224 & 0.6606179422267502 & 0.1812410855499991\end{array}$

$\begin{array}{llll}0.3986668806793361 & 0.9989709760423509 & 0.1812196720649979\end{array}$

0.40005143169764780 .32687828727475930 .1811291365750023

$\begin{array}{llll}0.1986046202569725 & 0.9974231925489931 & 0.1809514013825009\end{array}$

$\begin{array}{llll}0.9995513466306747 & 0.9974793079639639 & 0.1808750773374967\end{array}$

$\begin{array}{llll}0.3001250772143251 & 0.8306470167302393 & 0.1808491040974971\end{array}$

$\begin{array}{llll}0.0970718642359216 & 0.1643345836446173 & 0.1806310906500030\end{array}$

$\begin{array}{llll}0.2960588182135027 & 0.4904508576614504 & 0.1801666020825010\end{array}$

$\begin{array}{llll}0.5050961167782972 & 0.8383456542351695 & 0.1792713608150009\end{array}$

$\begin{array}{llll}0.6071663974712962 & 0.6666297093055391 & 0.1753513173900032\end{array}$

$\begin{array}{llll}0.5031693807408431 & 0.4918067634946723 & 0.1750172886800030\end{array}$

$\begin{array}{llll}0.3004906672772023 & 0.6123243114560069 & 0.1208860010500032\end{array}$

$\begin{array}{llll}0.3999341196350485 & 0.7778075147639072 & 0.1207323173450021\end{array}$

$\begin{array}{llll}0.8061617228198443 & 0.4412449012560842 & 0.1205337762149981\end{array}$

$\begin{array}{llll}0.9031653922156906 & 0.6101389366105465 & 0.1202765325650006\end{array}$

$\begin{array}{llll}0.7037627949494265 & 0.2755512228791233 & 0.1198205918449986\end{array}$

$\begin{array}{llll}0.9986557651380537 & 0.7772598406484263 & 0.1197936817999974\end{array}$

$\begin{array}{llll}0.7983812100601426 & 0.7738493474746804 & 0.1197068439075011\end{array}$

$\begin{array}{llll}0.4009202826271192 & 0.1149997877219491 & 0.1196841650300016\end{array}$

$\begin{array}{llll}0.0010549825246002 & 0.4447178795278148 & 0.1196805701374970\end{array}$

$\begin{array}{llll}0.5998359911495328 & 0.1127258864198311 & 0.1196156005024989\end{array}$

$\begin{array}{llll}0.1005148617550589 & 0.6128346097593038 & 0.1195919438775022\end{array}$

$\begin{array}{llll}0.7990277577843088 & 0.1079657998421695 & 0.1195216511800012\end{array}$

$0.6984520210975944 \quad 0.94406907383927320 .1195035291375035$

$\begin{array}{llll}0.8968713238654971 & 0.9402034566684208 & 0.1194828246599968\end{array}$

$\begin{array}{llll}0.5028816159308391 & 0.2801317116664492 & 0.1194272558299971\end{array}$

$\begin{array}{llll}0.2003576483734264 & 0.1114139349993408 & 0.1193559321424971\end{array}$

$\begin{array}{llll}0.9012196083242188 & 0.2749274021701993 & 0.1193404357150030\end{array}$

$\begin{array}{llll}0.2990756357572479 & 0.2782978950874622 & 0.1193286264674995\end{array}$

$\begin{array}{llll}0.0990948603403510 & 0.2773049943114572 & 0.1192754866600012\end{array}$

$\begin{array}{llll}0.0990386173387066 & 0.9438167176048893 & 0.1192373792874974\end{array}$

$\begin{array}{lllll}0.1977211910880285 & 0.4457067913586741 & 0.1190880634474993\end{array}$

$\begin{array}{llll}0.9985090520776367 & 0.1079510685584637 & 0.1189857300400021\end{array}$

$\begin{array}{llll}0.3003078689379421 & 0.9459002708601858 & 0.1189845379375001\end{array}$

$\begin{array}{llll}0.1996185754715682 & 0.7788035939168765 & 0.1189667343849976\end{array}$

$\begin{array}{llll}0.4984690304332986 & 0.9474919225371536 & 0.1189438320099967\end{array}$

$\begin{array}{llll}0.7062459488039892 & 0.6056166311192968 & 0.1185665616575022\end{array}$

$\begin{array}{lll}0.6080445039502465 & 0.4416126936143598 & 0.1183499418575025\end{array}$

$\begin{array}{llll}0.4998765685415520 & 0.6111377463369720 & 0.1182120422825008\end{array}$ 
0.39746207168534650 .44359488776140670 .1181596784375003

$\begin{array}{llll}0.5998926155959552 & 0.7795715729580408 & 0.1180175089900004\end{array}$

$\begin{array}{llll}0.0000000000000000 & 0.8888120000000015 & 0.0597319999999968\end{array}$

$\begin{array}{llll}0.1999939999999967 & 0.8888120000000015 & 0.0597319999999968\end{array}$

$\begin{array}{llll}0.3999870000000030 & 0.8888120000000015 & 0.0597319999999968\end{array}$

$\begin{array}{llll}0.0000000000000000 & 0.5555080000000032 & 0.0597319999999968\end{array}$

$\begin{array}{llll}0.1999939999999967 & 0.5555080000000032 & 0.0597319999999968\end{array}$

$\begin{array}{llll}0.0999970000000019 & 0.7221600000000024 & 0.0597319999999968\end{array}$

$\begin{array}{llll}0.0000000000000000 & 0.2222030000000004 & 0.0597319999999968\end{array}$

$\begin{array}{llll}0.5999809999999997 & 0.8888120000000015 & 0.0597319999999968\end{array}$

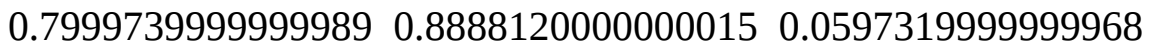

$\begin{array}{llll}0.3999870000000030 & 0.5555080000000032 & 0.0597319999999968\end{array}$

$\begin{array}{llll}0.5999809999999997 & 0.5555080000000032 & 0.0597319999999968\end{array}$

$\begin{array}{llll}0.4999839999999978 & 0.7221600000000024 & 0.0597319999999968\end{array}$

$\begin{array}{llll}0.1999939999999967 & 0.2222030000000004 & 0.0597319999999968\end{array}$

$\begin{array}{llll}0.3999870000000030 & 0.2222030000000004 & 0.0597319999999968\end{array}$

$\begin{array}{lllll}0.2999909999999986 & 0.3888549999999995 & 0.0597319999999968\end{array}$

$\begin{array}{lllll}0.2999909999999986 & 0.7221600000000024 & 0.0597319999999968\end{array}$

$\begin{array}{llll}0.0999970000000019 & 0.0555510000000012 & 0.0597319999999968\end{array}$

$\begin{array}{lll}0.0999970000000019 & 0.3888549999999995 & 0.0597319999999968\end{array}$

$\begin{array}{llll}0.7999739999999989 & 0.5555080000000032 & 0.0597319999999968\end{array}$

$\begin{array}{llll}0.8999710000000007 & 0.7221600000000024 & 0.0597319999999968\end{array}$

$\begin{array}{llll}0.5999809999999997 & 0.2222030000000004 & 0.0597319999999968\end{array}$

$\begin{array}{llll}0.7999739999999989 & 0.2222030000000004 & 0.0597319999999968\end{array}$

$\begin{array}{llll}0.6999769999999970 & 0.3888549999999995 & 0.0597319999999968\end{array}$

$\begin{array}{llll}0.6999769999999970 & 0.7221600000000024 & 0.0597319999999968\end{array}$

$\begin{array}{llll}0.4999839999999978 & 0.0555510000000012 & 0.0597319999999968\end{array}$

$\begin{array}{llll}0.4999839999999978 & 0.3888549999999995 & 0.0597319999999968\end{array}$

$\begin{array}{llll}0.2999909999999986 & 0.0555510000000012 & 0.0597319999999968\end{array}$

$\begin{array}{llll}0.8999710000000007 & 0.0555510000000012 & 0.0597319999999968\end{array}$

$\begin{array}{llll}0.8999710000000007 & 0.3888549999999995 & 0.0597319999999968\end{array}$

$\begin{array}{llll}0.6999769999999970 & 0.0555510000000012 & 0.0597319999999968\end{array}$

$\begin{array}{lll}0.0000000000000000 & 0.6666090000000011 & 0.0000000000000000\end{array}$

$\begin{array}{lll}0.1999939999999967 & 0.6666090000000011 & 0.0000000000000000\end{array}$

$\begin{array}{llll}0.3999870000000030 & 0.6666090000000011 & 0.0000000000000000\end{array}$

$\begin{array}{llll}0.2999909999999986 & 0.8332619999999977 & 0.0000000000000000\end{array}$

$\begin{array}{llll}0.0000000000000000 & 0.3333050000000028 & 0.0000000000000000\end{array}$

$\begin{array}{llll}0.1999939999999967 & 0.3333050000000028 & 0.0000000000000000\end{array}$

$\begin{array}{llll}0.0999970000000019 & 0.4999570000000020 & 0.0000000000000000\end{array}$

$\begin{array}{lllll}0.0999970000000019 & 0.8332619999999977 & 0.0000000000000000\end{array}$

$\begin{array}{llll}0.0000000000000000 & 0.0000000000000000 & 0.0000000000000000\end{array}$

0.59998099999999970 .66660900000000110 .0000000000000000

$\begin{array}{llll}0.7999739999999989 & 0.6666090000000011 & 0.0000000000000000\end{array}$

$\begin{array}{llll}0.6999769999999970 & 0.8332619999999977 & 0.0000000000000000\end{array}$

$\begin{array}{llll}0.3999870000000030 & 0.3333050000000028 & 0.0000000000000000\end{array}$

$\begin{array}{llll}0.5999809999999997 & 0.3333050000000028 & 0.0000000000000000\end{array}$

$\begin{array}{llll}0.4999839999999978 & 0.4999570000000020 & 0.0000000000000000\end{array}$ 
$\begin{array}{llll}0.4999839999999978 & 0.8332619999999977 & 0.0000000000000000\end{array}$

$\begin{array}{llll}0.1999939999999967 & 0.0000000000000000 & 0.0000000000000000\end{array}$

$\begin{array}{llll}0.3999870000000030 & 0.0000000000000000 & 0.0000000000000000\end{array}$

$\begin{array}{llll}0.2999909999999986 & 0.1666519999999991 & 0.0000000000000000\end{array}$

$\begin{array}{lll}0.2999909999999986 & 0.4999570000000020 & 0.0000000000000000\end{array}$

$\begin{array}{llll}0.0999970000000019 & 0.1666519999999991 & 0.0000000000000000\end{array}$

$\begin{array}{lll}0.7999739999999989 & 0.3333050000000028 & 0.0000000000000000\end{array}$

$\begin{array}{llll}0.8999710000000007 & 0.4999570000000020 & 0.0000000000000000\end{array}$

$\begin{array}{llll}0.8999710000000007 & 0.8332619999999977 & 0.0000000000000000\end{array}$

$\begin{array}{lll}0.5999809999999997 & 0.0000000000000000 & 0.0000000000000000\end{array}$

$\begin{array}{llll}0.7999739999999989 & 0.0000000000000000 & 0.0000000000000000\end{array}$

$\begin{array}{llll}0.6999769999999970 & 0.1666519999999991 & 0.0000000000000000\end{array}$

$\begin{array}{llll}0.6999769999999970 & 0.4999570000000020 & 0.0000000000000000\end{array}$

$\begin{array}{llll}0.4999839999999978 & 0.1666519999999991 & 0.0000000000000000\end{array}$

$\begin{array}{llll}0.8999710000000007 & 0.1666519999999991 & 0.0000000000000000\end{array}$

$\begin{array}{llll}0.3372147649398585 & 0.5217103123766904 & 0.2516010248300020\end{array}$

$\begin{array}{llll}0.5028513725669796 & 0.7913227855780605 & 0.2504553876924973\end{array}$

$\begin{array}{llll}0.6588354565062886 & 0.7045822853610417 & 0.2549628701825029\end{array}$

$\begin{array}{llll}0.4920020421405127 & 0.4316728697422079 & 0.2537060958674999\end{array}$

$\begin{array}{llll}0.5832482676325839 & 0.5636711081086432 & 0.2559974126699984\end{array}$

$\begin{array}{lllll}0.5833131662520472 & 0.6556433990201214 & 0.2545125756849984\end{array}$

$\begin{array}{lllll}0.4998286153157494 & 0.5189735391227117 & 0.2540100907449983\end{array}$

$\begin{array}{llll}0.4083557270229647 & 0.5675161335262402 & 0.2509327225849987\end{array}$

$\begin{array}{llll}0.4952674590418269 & 0.7091096241286365 & 0.2505584555375009\end{array}$

$\begin{array}{llll}0.4119352557203371 & 0.6609334175391268 & 0.2455151944050016\end{array}$

$\begin{array}{llll}0.6474719330576804 & 0.5272913153557823 & 0.2576297622349983\end{array}$

$\begin{array}{llll}0.7221063557476768 & 0.6780236443706400 & 0.2523383586500003\end{array}$

$\begin{array}{llll}0.6502762974644583 & 0.7704819274365349 & 0.2508966900999994\end{array}$

$\begin{array}{llll}0.5468534291074363 & 0.3917172985860873 & 0.2502874006350027\end{array}$

$\begin{array}{llll}0.4279198806109861 & 0.4069994058332256 & 0.2496652983625012\end{array}$

$\begin{array}{llll}0.3479203962342794 & 0.6975303059515952 & 0.2471751813899985\end{array}$

Zwitterion on $\mathrm{Cu}$ - VASP - revPBE+D3-calculated lattice - Preferred Epitaxy ( $\underline{\mathrm{H}}_{\mathrm{hcp}}-0$ site)

$\mathrm{Cu} \mathrm{O} \mathrm{N} \mathrm{C} \mathrm{H}$

1.00000000000000

$\begin{array}{lll}12.5239999999999991 & 0.0000000000000000 & 0.0000000000000000\end{array}$

$\begin{array}{lll}0.0000000000000000 & 13.0160000000000000 & 0.0000000000000000\end{array}$

$0.0000000000000000 \quad 0.0000000000000000 \quad 40.0000000000000000$

$\mathrm{Cu} O \mathrm{~N}$ C $\mathrm{H}$

$\begin{array}{lllll}120 & 2 & 2 & 6 & 6\end{array}$

Direct

$0.0000000000000000 \quad 0.66664105716041890 .0000000000000000$

$\begin{array}{lll}0.2000159693388710 & 0.6666410571604189 & 0.0000000000000000\end{array}$

$\begin{array}{llll}0.3999520919833941 & 0.6666410571604189 & 0.0000000000000000\end{array}$

$\begin{array}{llll}0.2999840306611290 & 0.8332821143208378 & 0.0000000000000000\end{array}$

$\begin{array}{llll}0.0000000000000000 & 0.3332821143208378 & 0.0000000000000000\end{array}$

$\begin{array}{llll}0.2000159693388710 & 0.3332821143208378 & 0.0000000000000000\end{array}$ 
$\begin{array}{lll}0.0999680613222580 & 0.4999231714812566 & 0.0000000000000000\end{array}$

$\begin{array}{llll}0.0999680613222580 & 0.8332821143208378 & 0.0000000000000000\end{array}$

$\begin{array}{llll}0.0000000000000000 & 0.0000000000000000 & 0.0000000000000000\end{array}$

$\begin{array}{lll}0.5999680613222580 & 0.6666410571604189 & 0.0000000000000000\end{array}$

$\begin{array}{lll}0.7999840306611290 & 0.6666410571604189 & 0.0000000000000000\end{array}$

$\begin{array}{llll}0.7000159693388710 & 0.8332821143208378 & 0.0000000000000000\end{array}$

$\begin{array}{llll}0.3999520919833941 & 0.3332821143208378 & 0.0000000000000000\end{array}$

$\begin{array}{llll}0.5999680613222580 & 0.3332821143208378 & 0.0000000000000000\end{array}$

$\begin{array}{llll}0.5000000000000000 & 0.4999231714812566 & 0.0000000000000000\end{array}$

$\begin{array}{lll}0.5000000000000000 & 0.8332821143208378 & 0.0000000000000000\end{array}$

$\begin{array}{llll}0.2000159693388710 & 0.0000000000000000 & 0.0000000000000000\end{array}$

$\begin{array}{llll}0.3999520919833941 & 0.0000000000000000 & 0.0000000000000000\end{array}$

$\begin{array}{llll}0.2999840306611290 & 0.1666410571604189 & 0.0000000000000000\end{array}$

$\begin{array}{lll}0.2999840306611290 & 0.4999231714812566 & 0.0000000000000000\end{array}$

$\begin{array}{llll}0.0999680613222580 & 0.1666410571604189 & 0.0000000000000000\end{array}$

$\begin{array}{llll}0.7999840306611290 & 0.3332821143208378 & 0.0000000000000000\end{array}$

$\begin{array}{llll}0.8999520919833941 & 0.4999231714812566 & 0.0000000000000000\end{array}$

$\begin{array}{llll}0.8999520919833941 & 0.8332821143208378 & 0.0000000000000000\end{array}$

$\begin{array}{lll}0.5999680613222580 & 0.0000000000000000 & 0.0000000000000000\end{array}$

$\begin{array}{llll}0.7999840306611290 & 0.0000000000000000 & 0.0000000000000000\end{array}$

$\begin{array}{llll}0.7000159693388710 & 0.1666410571604189 & 0.0000000000000000\end{array}$

$\begin{array}{llll}0.7000159693388710 & 0.4999231714812566 & 0.0000000000000000\end{array}$

$\begin{array}{llll}0.5000000000000000 & 0.1666410571604189 & 0.0000000000000000\end{array}$

$\begin{array}{lll}0.8999520919833941 & 0.1666410571604189 & 0.0000000000000000\end{array}$

$\begin{array}{llll}0.0000000000000000 & 0.8888291333743084 & 0.0511249999999990\end{array}$

$\begin{array}{llll}0.2000159693388710 & 0.8888291333743084 & 0.0511249999999990\end{array}$

$\begin{array}{llll}0.3999520919833941 & 0.8888291333743084 & 0.0511249999999990\end{array}$

$\begin{array}{llll}0.0000000000000000 & 0.5554701905347272 & 0.0511249999999990\end{array}$

$\begin{array}{llll}0.2000159693388710 & 0.5554701905347272 & 0.0511249999999990\end{array}$

$\begin{array}{llll}0.0999680613222580 & 0.7221880762138895 & 0.0511249999999990\end{array}$

$\begin{array}{llll}0.0000000000000000 & 0.2221880762138895 & 0.0511249999999990\end{array}$

$\begin{array}{llll}0.5999680613222580 & 0.8888291333743084 & 0.0511249999999990\end{array}$

$\begin{array}{llll}0.7999840306611290 & 0.8888291333743084 & 0.0511249999999990\end{array}$

$\begin{array}{llll}0.3999520919833941 & 0.5554701905347272 & 0.0511249999999990\end{array}$

$\begin{array}{llll}0.5999680613222580 & 0.5554701905347272 & 0.0511249999999990\end{array}$

$\begin{array}{llll}0.5000000000000000 & 0.7221880762138895 & 0.0511249999999990\end{array}$

$\begin{array}{llll}0.2000159693388710 & 0.2221880762138895 & 0.0511249999999990\end{array}$

$\begin{array}{llll}0.3999520919833941 & 0.2221880762138895 & 0.0511249999999990\end{array}$

$\begin{array}{llll}0.2999840306611290 & 0.3888291333743084 & 0.0511249999999990\end{array}$

$\begin{array}{llll}0.2999840306611290 & 0.7221880762138895 & 0.0511249999999990\end{array}$

$\begin{array}{llll}0.0999680613222580 & 0.0555470190534706 & 0.0511249999999990\end{array}$

$\begin{array}{llll}0.0999680613222580 & 0.3888291333743084 & 0.0511249999999990\end{array}$

$\begin{array}{llll}0.7999840306611290 & 0.5554701905347272 & 0.0511249999999990\end{array}$

$\begin{array}{llll}0.8999520919833941 & 0.7221880762138895 & 0.0511249999999990\end{array}$

$\begin{array}{llll}0.5999680613222580 & 0.2221880762138895 & 0.0511249999999990\end{array}$

$\begin{array}{llll}0.7999840306611290 & 0.2221880762138895 & 0.0511249999999990\end{array}$

$\begin{array}{llll}0.7000159693388710 & 0.3888291333743084 & 0.0511249999999990\end{array}$ 
$\begin{array}{lll}0.7000159693388710 & 0.7221880762138895 & 0.0511249999999990\end{array}$

$\begin{array}{llll}0.5000000000000000 & 0.0555470190534706 & 0.0511249999999990\end{array}$

$\begin{array}{llll}0.5000000000000000 & 0.3888291333743084 & 0.0511249999999990\end{array}$

$\begin{array}{llll}0.2999840306611290 & 0.0555470190534706 & 0.0511249999999990\end{array}$

$\begin{array}{llll}0.8999520919833941 & 0.0555470190534706 & 0.0511249999999990\end{array}$

$\begin{array}{llll}0.8999520919833941 & 0.3888291333743084 & 0.0511249999999990\end{array}$

$\begin{array}{llll}0.7000159693388710 & 0.0555470190534706 & 0.0511249999999990\end{array}$

$\begin{array}{llll}0.4998455594259283 & 0.6106466200336058 & 0.1014019834553253\end{array}$

$\begin{array}{llll}0.3989163823691726 & 0.7788292289739260 & 0.1017853652160241\end{array}$

$\begin{array}{llll}0.6009343184024090 & 0.7786620611371902 & 0.1017540129546575\end{array}$

$\begin{array}{llll}0.7009770461466545 & 0.6104878735380268 & 0.1027933060481629\end{array}$

$\begin{array}{llll}0.2987009087286125 & 0.6104454942756448 & 0.1025195873441689\end{array}$

$\begin{array}{llll}0.1995920873068613 & 0.4444415464402240 & 0.1023826195382753\end{array}$

$\begin{array}{llll}0.8003440228672289 & 0.4444143549642519 & 0.1022626589743894\end{array}$

$\begin{array}{llll}0.3986533976651145 & 0.4442056301725543 & 0.1023054745634582\end{array}$

$\begin{array}{llll}0.6011029733465989 & 0.4441461282732533 & 0.1022332637159451\end{array}$

$\begin{array}{llll}0.9004195916964761 & 0.2774832267819320 & 0.1021690462360212\end{array}$

$\begin{array}{lllll}0.0996412386328984 & 0.2775389631165694 & 0.1021921556359260\end{array}$

$\begin{array}{llll}0.4998786537502440 & 0.9452126703407799 & 0.1020668795240312\end{array}$

$\begin{array}{llll}0.8000986072466318 & 0.1109414434272011 & 0.1022773542863717\end{array}$

$\begin{array}{llll}0.1998696060614265 & 0.1109366017729414 & 0.1022884224015357\end{array}$

$\begin{array}{llll}0.9999883770607951 & 0.1108543235279669 & 0.1022802072376905\end{array}$

$\begin{array}{llll}0.7002398332228097 & 0.9442905049274160 & 0.1025727375371446\end{array}$

$\begin{array}{llll}0.2996300309157007 & 0.9440067026678989 & 0.1027729441492298\end{array}$

$\begin{array}{llll}0.0999048108323350 & 0.9443822461150120 & 0.1022809390507575\end{array}$

$\begin{array}{llll}0.8999611401991032 & 0.9443319897729694 & 0.1022688989595508\end{array}$

$\begin{array}{llll}0.6000625441059242 & 0.1112631136515319 & 0.1022415624128712\end{array}$

0.39974571402481730 .11119935636678240 .1022418140407382

$\begin{array}{llll}0.2993802422541094 & 0.2774934824035071 & 0.1021721444167625\end{array}$

$\begin{array}{llll}0.7005246381914247 & 0.2774186346930279 & 0.1021236925788500\end{array}$

$\begin{array}{llll}0.7998744120903893 & 0.7774445991873478 & 0.1029424116893765\end{array}$

$\begin{array}{llll}0.9998603558960539 & 0.4443801623819326 & 0.1020922736945664\end{array}$

$\begin{array}{llll}0.2000981924485643 & 0.7778924848420417 & 0.1028575466767450\end{array}$

$\begin{array}{llll}0.9998444402883493 & 0.7776606992424178 & 0.1022631130600047\end{array}$

$\begin{array}{llll}0.4998045618968305 & 0.2773549503678154 & 0.1018935026055061\end{array}$

$\begin{array}{llll}0.8992743128820706 & 0.6116826423627089 & 0.1026962565980796\end{array}$

$\begin{array}{llll}0.1000005236846278 & 0.6114924210884993 & 0.1024939646106731\end{array}$

0.59839226994611750 .66801236355341590 .1514165550721245

0.40050925601725140 .66784002999877150 .1511972686027576

$\begin{array}{llll}0.2964956495105326 & 0.4976612637572941 & 0.1548982954295823\end{array}$

$\begin{array}{llll}0.7034660026221076 & 0.4967840513631145 & 0.1546177374922948\end{array}$

$\begin{array}{llll}0.4999732227866573 & 0.4975271388662748 & 0.1520376661550742\end{array}$

$\begin{array}{llll}0.5002415274866721 & 0.8355866718600282 & 0.1525248035548188\end{array}$

$\begin{array}{llll}0.0997098008769584 & 0.1665722282411082 & 0.1545324895030262\end{array}$

$\begin{array}{llll}0.9003728462684819 & 0.1665214968869222 & 0.1545313144199980\end{array}$

$\begin{array}{llll}0.1995229982062554 & 0.0003311404739321 & 0.1545319256876567\end{array}$

$\begin{array}{llll}0.8004443945664548 & 0.0004917085156393 & 0.1544888751976288\end{array}$ 
$\begin{array}{llll}0.0000035252791761 & 0.0004677290044697 & 0.1544671111436102\end{array}$

$\begin{array}{llll}0.0000032050031464 & 0.3332749279967118 & 0.1544630982295843\end{array}$

0.70062031760491550 .83490892159653640 .1553330475076322

$\begin{array}{llll}0.2995726631742244 & 0.8342869177960921 & 0.1561678142034850\end{array}$

$\begin{array}{llll}0.5999862055337104 & 0.0006325508399527 & 0.1543591531482065\end{array}$

$\begin{array}{llll}0.3999473692886113 & 0.0004985558899122 & 0.1543845092408773\end{array}$

$\begin{array}{llll}0.2997469225489908 & 0.1664332670890332 & 0.1545882346378775\end{array}$

$\begin{array}{llll}0.7001601997573772 & 0.1663645725253105 & 0.1545677440121267\end{array}$

$\begin{array}{llll}0.1991359001489030 & 0.3318771148383277 & 0.1542078906568136\end{array}$

$\begin{array}{llll}0.8009530461546106 & 0.3314449768305892 & 0.1540449119140419\end{array}$

$\begin{array}{llll}0.9009898419923275 & 0.8342194572124413 & 0.1544072466463575\end{array}$

$\begin{array}{llll}0.0989508263039980 & 0.8341362434304216 & 0.1543929679874836\end{array}$

$\begin{array}{llll}0.4998315064377010 & 0.1660123426709461 & 0.1546015276006614\end{array}$

$0.4000113521060698 \quad 0.33083238368419640 .1536743122357507$

$\begin{array}{llll}0.5996887085407820 & 0.3303591481469752 & 0.1534978456956466\end{array}$

$\begin{array}{llll}0.0982416254296226 & 0.4999136368037185 & 0.1542945894344285\end{array}$

$\begin{array}{llll}0.8003967998060944 & 0.6676879016256123 & 0.1562850249744514\end{array}$

$\begin{array}{llll}0.9014341014227227 & 0.4999301706412140 & 0.1542498022988639\end{array}$

0.19850726447234020 .66732586386111590 .1551554662251320

$\begin{array}{llll}0.9997274320290686 & 0.6669222191306048 & 0.1548000987368141\end{array}$

$\begin{array}{llll}0.3115997454130195 & 0.7167178176351783 & 0.2083224740730714\end{array}$

$\begin{array}{llll}0.7002942954802549 & 0.7080715736021475 & 0.2096120466124560\end{array}$

$\begin{array}{llll}0.3038591366645420 & 0.5064435475269349 & 0.2090648945683995\end{array}$

0.69814309088756230 .49821639543480250 .2088568264552606

$\begin{array}{llll}0.5063070166584538 & 0.7174620320433860 & 0.2106680529264224\end{array}$

$\begin{array}{lllll}0.4042703800397049 & 0.5560792403711901 & 0.2132009933197878\end{array}$

$0.4033959313877844 \quad 0.66741170769162750 .2111537776190744$

$\begin{array}{llll}0.6000564240119317 & 0.5513945347980354 & 0.2131004042378066\end{array}$

$\begin{array}{llll}0.5008158590862521 & 0.5000209473080801 & 0.2143201979470817\end{array}$

$\begin{array}{llll}0.6065127269299495 & 0.6628423278003694 & 0.2115824793691985\end{array}$

$\begin{array}{llll}0.5087282473323640 & 0.8011972625327104 & 0.2126163224729751\end{array}$

$\begin{array}{llll}0.4987682829152078 & 0.4160840186851686 & 0.2155454530796348\end{array}$

$\begin{array}{llll}0.7618640037755426 & 0.5412970344597241 & 0.2164885235096321\end{array}$

$\begin{array}{llll}0.7005474161571925 & 0.4244930026478002 & 0.2172246845744041\end{array}$

$\begin{array}{llll}0.2986450702699983 & 0.4335496540614798 & 0.2181225580760042\end{array}$

$\begin{array}{llll}0.2419699199451699 & 0.5522477943718371 & 0.2164884235323044\end{array}$

Zwitterion on $\mathrm{Cu}$ - VASP - revPBE +D3-calculated lattice - $\left(\mathrm{H}_{\mathrm{hcp}}-30\right.$ site $)$ $\mathrm{Cu} \mathrm{O} \mathrm{N} \mathrm{C} \mathrm{H}$

1.00000000000000

$\begin{array}{lll}12.5239999999999991 & 0.0000000000000000 & 0.0000000000000000\end{array}$

$\begin{array}{lll}0.0000000000000000 & 13.0160000000000000 & 0.0000000000000000\end{array}$

$0.0000000000000000 \quad 0.0000000000000000 \quad 40.0000000000000000$

$\begin{array}{llllll}\mathrm{Cu} & \mathrm{O} & \mathrm{N} & \mathrm{C} & \mathrm{H}\end{array}$

$\begin{array}{lllll}120 & 2 & 2 & 6 & 6\end{array}$

Direct

$0.0000000000000000 \quad 0.66664105716041890 .0000000000000000$ 
$\begin{array}{lll}0.2000159693388710 & 0.6666410571604189 & 0.0000000000000000\end{array}$

$\begin{array}{llll}0.3999520919833941 & 0.6666410571604189 & 0.0000000000000000\end{array}$

$\begin{array}{llll}0.2999840306611290 & 0.8332821143208378 & 0.0000000000000000\end{array}$

$\begin{array}{llll}0.0000000000000000 & 0.3332821143208378 & 0.0000000000000000\end{array}$

$\begin{array}{llll}0.2000159693388710 & 0.3332821143208378 & 0.0000000000000000\end{array}$

$\begin{array}{llll}0.0999680613222580 & 0.4999231714812566 & 0.0000000000000000\end{array}$

$\begin{array}{llll}0.0999680613222580 & 0.8332821143208378 & 0.0000000000000000\end{array}$

$\begin{array}{llll}0.0000000000000000 & 0.0000000000000000 & 0.0000000000000000\end{array}$

$\begin{array}{lll}0.5999680613222580 & 0.6666410571604189 & 0.0000000000000000\end{array}$

$\begin{array}{lll}0.7999840306611290 & 0.6666410571604189 & 0.0000000000000000\end{array}$

$\begin{array}{llll}0.7000159693388710 & 0.8332821143208378 & 0.0000000000000000\end{array}$

$\begin{array}{llll}0.3999520919833941 & 0.3332821143208378 & 0.0000000000000000\end{array}$

$\begin{array}{llll}0.5999680613222580 & 0.3332821143208378 & 0.0000000000000000\end{array}$

$\begin{array}{llll}0.5000000000000000 & 0.4999231714812566 & 0.0000000000000000\end{array}$

$\begin{array}{llll}0.5000000000000000 & 0.8332821143208378 & 0.0000000000000000\end{array}$

$\begin{array}{llll}0.2000159693388710 & 0.0000000000000000 & 0.0000000000000000\end{array}$

$\begin{array}{llll}0.3999520919833941 & 0.0000000000000000 & 0.0000000000000000\end{array}$

$\begin{array}{llll}0.2999840306611290 & 0.1666410571604189 & 0.0000000000000000\end{array}$

$\begin{array}{llll}0.2999840306611290 & 0.4999231714812566 & 0.0000000000000000\end{array}$

$\begin{array}{llll}0.0999680613222580 & 0.1666410571604189 & 0.0000000000000000\end{array}$

$\begin{array}{llll}0.7999840306611290 & 0.3332821143208378 & 0.0000000000000000\end{array}$

$\begin{array}{llll}0.8999520919833941 & 0.4999231714812566 & 0.0000000000000000\end{array}$

$\begin{array}{llll}0.8999520919833941 & 0.8332821143208378 & 0.0000000000000000\end{array}$

$\begin{array}{llll}0.5999680613222580 & 0.0000000000000000 & 0.0000000000000000\end{array}$

$\begin{array}{lll}0.7999840306611290 & 0.0000000000000000 & 0.0000000000000000\end{array}$

$\begin{array}{llll}0.7000159693388710 & 0.1666410571604189 & 0.0000000000000000\end{array}$

$\begin{array}{llll}0.7000159693388710 & 0.4999231714812566 & 0.0000000000000000\end{array}$

$\begin{array}{llll}0.5000000000000000 & 0.1666410571604189 & 0.0000000000000000\end{array}$

$\begin{array}{lll}0.8999520919833941 & 0.1666410571604189 & 0.0000000000000000\end{array}$

$\begin{array}{llll}0.0000000000000000 & 0.8888291333743084 & 0.0511249999999990\end{array}$

$\begin{array}{llll}0.2000159693388710 & 0.8888291333743084 & 0.0511249999999990\end{array}$

$\begin{array}{llll}0.3999520919833941 & 0.8888291333743084 & 0.0511249999999990\end{array}$

$\begin{array}{llll}0.0000000000000000 & 0.5554701905347272 & 0.0511249999999990\end{array}$

$\begin{array}{llll}0.2000159693388710 & 0.5554701905347272 & 0.0511249999999990\end{array}$

$\begin{array}{llll}0.0999680613222580 & 0.7221880762138895 & 0.0511249999999990\end{array}$

$\begin{array}{llll}0.0000000000000000 & 0.2221880762138895 & 0.0511249999999990\end{array}$

$\begin{array}{llll}0.5999680613222580 & 0.8888291333743084 & 0.0511249999999990\end{array}$

$\begin{array}{llll}0.7999840306611290 & 0.8888291333743084 & 0.0511249999999990\end{array}$

$\begin{array}{llll}0.3999520919833941 & 0.5554701905347272 & 0.0511249999999990\end{array}$

$\begin{array}{llll}0.5999680613222580 & 0.5554701905347272 & 0.0511249999999990\end{array}$

$\begin{array}{llll}0.5000000000000000 & 0.7221880762138895 & 0.0511249999999990\end{array}$

$\begin{array}{llll}0.2000159693388710 & 0.2221880762138895 & 0.0511249999999990\end{array}$

$\begin{array}{llll}0.3999520919833941 & 0.2221880762138895 & 0.0511249999999990\end{array}$

$\begin{array}{llll}0.2999840306611290 & 0.3888291333743084 & 0.0511249999999990\end{array}$

$\begin{array}{llll}0.2999840306611290 & 0.7221880762138895 & 0.0511249999999990\end{array}$

$\begin{array}{llll}0.0999680613222580 & 0.0555470190534706 & 0.0511249999999990\end{array}$

$\begin{array}{llll}0.0999680613222580 & 0.3888291333743084 & 0.0511249999999990\end{array}$ 
$\begin{array}{llll}0.7999840306611290 & 0.5554701905347272 & 0.0511249999999990\end{array}$

$\begin{array}{llll}0.8999520919833941 & 0.7221880762138895 & 0.0511249999999990\end{array}$

$\begin{array}{llll}0.5999680613222580 & 0.2221880762138895 & 0.0511249999999990\end{array}$

$\begin{array}{llll}0.7999840306611290 & 0.2221880762138895 & 0.0511249999999990\end{array}$

$\begin{array}{llll}0.7000159693388710 & 0.3888291333743084 & 0.0511249999999990\end{array}$

$\begin{array}{llll}0.7000159693388710 & 0.7221880762138895 & 0.0511249999999990\end{array}$

$\begin{array}{llll}0.5000000000000000 & 0.0555470190534706 & 0.0511249999999990\end{array}$

$\begin{array}{llll}0.5000000000000000 & 0.3888291333743084 & 0.0511249999999990\end{array}$

$\begin{array}{llll}0.2999840306611290 & 0.0555470190534706 & 0.0511249999999990\end{array}$

$\begin{array}{llll}0.8999520919833941 & 0.0555470190534706 & 0.0511249999999990\end{array}$

$\begin{array}{llll}0.8999520919833941 & 0.3888291333743084 & 0.0511249999999990\end{array}$

$\begin{array}{llll}0.7000159693388710 & 0.0555470190534706 & 0.0511249999999990\end{array}$

$\begin{array}{llll}0.4993793560627502 & 0.6110178761314594 & 0.1012270534885165\end{array}$

$\begin{array}{llll}0.3997520050114942 & 0.7787457528578732 & 0.1018389776617876\end{array}$

$\begin{array}{llll}0.6006928662055658 & 0.7795753617871752 & 0.1019365148743928\end{array}$

$\begin{array}{llll}0.7014128273792191 & 0.6111106941147921 & 0.1014273213930043\end{array}$

0.29951299992517730 .61223402704030860 .1025314209234980

$\begin{array}{llll}0.1991292513435967 & 0.4442763423663661 & 0.1019377589013277\end{array}$

$0.8012714962652436 \quad 0.44371762849455690 .1017524520741465$

$0.3995333660494608 \quad 0.44410841007133690 .1022331044096492$

$0.6001282551926254 \quad 0.44350033682555620 .1015236441828002$

$\begin{array}{llll}0.9001771646703904 & 0.2771461476106681 & 0.1022426488580564\end{array}$

$\begin{array}{llll}0.0995138512668450 & 0.2772944847608235 & 0.1021685601563436\end{array}$

$\begin{array}{llll}0.4999954347796862 & 0.9454569231543631 & 0.1021565636447755\end{array}$

$\begin{array}{llll}0.8000086850441621 & 0.1107167988347808 & 0.1023118169056894\end{array}$

$\begin{array}{lll}0.1996486676408234 & 0.1110570173987319 & 0.1022703151793709\end{array}$

$\begin{array}{llll}-0.0001747564032759 & 0.1105222404833312 & 0.1022628721312184\end{array}$

0.70016299804746650 .94375735473312590 .1030046129005522

$\begin{array}{llll}0.2994265203902806 & 0.9449599134835331 & 0.1021183240529317\end{array}$

$\begin{array}{lllll}0.0997063693831101 & 0.9439698159373957 & 0.1022626780267668\end{array}$

$\begin{array}{llll}0.8997580502911687 & 0.9439119762674896 & 0.1023384808304556\end{array}$

$\begin{array}{llll}0.5999310007539208 & 0.1114007783420346 & 0.1022283616955152\end{array}$

$\begin{array}{llll}0.3997051750734635 & 0.1114189583034425 & 0.1022725324587743\end{array}$

$\begin{array}{llll}0.2999981646417798 & 0.2777271110340810 & 0.1024553318372019\end{array}$

$\begin{array}{llll}0.7003740156760110 & 0.2771242777022450 & 0.1020925804237645\end{array}$

$\begin{array}{llll}0.7993372412978481 & 0.7777956419228222 & 0.1031958711407808\end{array}$

$\begin{array}{llll}1.0001908073343910 & 0.4442805163598628 & 0.1021186305092782\end{array}$

$\begin{array}{lll}0.1996321996930029 & 0.7765050546737418 & 0.1029891802815056\end{array}$

$\begin{array}{llll}0.9998033162398942 & 0.7775001930835809 & 0.1023152935285603\end{array}$

$\begin{array}{llll}0.4990456309597545 & 0.2778653003968086 & 0.1025725050985720\end{array}$

$\begin{array}{llll}0.9005617008409815 & 0.6111763029625484 & 0.1022419250708748\end{array}$

$\begin{array}{lll}0.1008266570442813 & 0.6115956836984742 & 0.1029584550154829\end{array}$

$\begin{array}{llll}0.6011544271734574 & 0.6664899137901981 & 0.1500903571946899\end{array}$

$0.40303571011193730 .6698349272270554 \quad 0.1522876652997913$

$\begin{array}{llll}0.2967754799697349 & 0.4998940168111578 & 0.1521770321462819\end{array}$

0.70208190774792330 .49756959404229760 .1518238658727318

$\begin{array}{llll}0.5030466700652295 & 0.4994210765945769 & 0.1520207340484508\end{array}$ 
$\begin{array}{llll}0.4989984260433541 & 0.8377759776206748 & 0.1530432788508886\end{array}$

$\begin{array}{llll}0.0994044420289272 & 0.1664857642867318 & 0.1544549871265870\end{array}$

$\begin{array}{llll}0.8999788233706831 & 0.1664577143014223 & 0.1544055305022018\end{array}$

$\begin{array}{llll}0.1992894615369260 & 0.0003760598051039 & 0.1544443692351943\end{array}$

$0.8000710132545569-0.00010868204039610 .1545690998237240$

$-0.0003445340242114-0.00027408194738700 .1543901641913116$

$\begin{array}{llll}-0.0005730901679850 & 0.3328463725134504 & 0.1545215527268354\end{array}$

$\begin{array}{llll}0.6988785131350429 & 0.8324356160274565 & 0.1578403583327177\end{array}$

0.29879306515585220 .83509630812935690 .1536314033217846

$\begin{array}{llll}0.5996896191963541 & 0.0018593006508861 & 0.1542906893152615\end{array}$

$\begin{array}{llll}0.3989935088116183 & 0.0015347064749210 & 0.1543651652841656\end{array}$

$0.2993627748300904 \quad 0.16687157609894440 .1545400186159741$

$\begin{array}{llll}0.6999986181198624 & 0.1667011655457860 & 0.1544522301882520\end{array}$

$\begin{array}{llll}0.1983946707517717 & 0.3328581821061076 & 0.1540508405904225\end{array}$

$\begin{array}{llll}0.8002593127827887 & 0.3328818986515474 & 0.1544647494195033\end{array}$

$\begin{array}{llll}0.8997869978092137 & 0.8330116881939067 & 0.1547186103109345\end{array}$

$\begin{array}{llll}0.0992621168402294 & 0.8333619994086288 & 0.1544897191672928\end{array}$

0.49927836172114890 .16761216202289490 .1546543522295051

$\begin{array}{llll}0.3981552459739540 & 0.3340369783883767 & 0.1554753040432457\end{array}$

$\begin{array}{llll}0.6004702620952564 & 0.3322310706726686 & 0.1538045517007764\end{array}$

$\begin{array}{lll}0.0980555478653292 & 0.4992381306490424 & 0.1543367624480404\end{array}$

$\begin{array}{llll}0.8009242999826663 & 0.6648804982629718 & 0.1546354269004938\end{array}$

0.90050888845071720 .49922503517689290 .1543054570298643

$\begin{array}{llll}0.2001576401892961 & 0.6668024542619893 & 0.1579759455423684\end{array}$

$\begin{array}{llll}0.9998688753525058 & 0.6664946227114485 & 0.1546914811758079\end{array}$

$\begin{array}{llll}0.2767842689899402 & 0.6033159570592085 & 0.2077555002468579\end{array}$

$\begin{array}{llll}0.6152518610916459 & 0.7874734804944235 & 0.2093386756742828\end{array}$

$\begin{array}{llll}0.3808938740943725 & 0.4190392655935942 & 0.2129941991550565\end{array}$

$\begin{array}{llll}0.7243518497908551 & 0.6063087959434343 & 0.2136734121639907\end{array}$

$\begin{array}{llll}0.4432706469854935 & 0.7003133021292912 & 0.2097836218016720\end{array}$

$\begin{array}{llll}0.4409579535103698 & 0.5108229490741465 & 0.2136963578763567\end{array}$

0.38171291450855480 .60684988965011930 .2105283248516853

$\begin{array}{llll}0.6128297577494954 & 0.6039703743092304 & 0.2145724583443558\end{array}$

$\begin{array}{lll}0.5535331333833715 & 0.5110324960058329 & 0.2153557682627565\end{array}$

$\begin{array}{llll}0.5590326313443071 & 0.7023971148186626 & 0.2115111195599216\end{array}$

$\begin{array}{llll}0.4010200851340352 & 0.7734294538153146 & 0.2120079416072966\end{array}$

$\begin{array}{llll}0.5958056169512300 & 0.4376923803342211 & 0.2169181044818083\end{array}$

$\begin{array}{llll}0.7572414948252784 & 0.6748886599576792 & 0.2201798936720416\end{array}$

$\begin{array}{llll}0.7662082124831466 & 0.5422355986310839 & 0.2195953021489641\end{array}$

$\begin{array}{llll}0.4135261391500094 & 0.3558896871352900 & 0.2237761714030878\end{array}$

$\begin{array}{lll}0.3019424790088219 & 0.4300332817424254 & 0.2181042697996203\end{array}$

Zwitterion on $\mathrm{Cu}$ - VASP - revPBE $+\mathrm{D} 3$-calculated lattice $-\left(\mathrm{H}_{\mathrm{hcp}}-60\right.$ site $)$

$\mathrm{Cu} \mathrm{O} \mathrm{N} \mathrm{C} \mathrm{H}$

1.00000000000000

$\begin{array}{lll}12.5239999999999991 & 0.0000000000000000 & 0.0000000000000000\end{array}$

$\begin{array}{llll}0.0000000000000000 & 13.0160000000000000 & 0.0000000000000000\end{array}$ 

0.0000000000000000

$\mathrm{Cu} \quad \mathrm{O} \quad \mathrm{N} \quad \mathrm{C} \quad \mathrm{H}$

$\begin{array}{lllll}120 & 2 & 2 & 6 & 6\end{array}$

Direct

$\begin{array}{llll}0.0000000000000000 & 0.6666410571604189 & 0.0000000000000000\end{array}$

$\begin{array}{llll}0.2000159693388710 & 0.6666410571604189 & 0.0000000000000000\end{array}$

$\begin{array}{llll}0.3999520919833941 & 0.6666410571604189 & 0.0000000000000000\end{array}$

$\begin{array}{llll}0.2999840306611290 & 0.8332821143208378 & 0.0000000000000000\end{array}$

$\begin{array}{llll}0.0000000000000000 & 0.3332821143208378 & 0.0000000000000000\end{array}$

$\begin{array}{llll}0.2000159693388710 & 0.3332821143208378 & 0.0000000000000000\end{array}$

$\begin{array}{llll}0.0999680613222580 & 0.4999231714812566 & 0.0000000000000000\end{array}$

$\begin{array}{llll}0.0999680613222580 & 0.8332821143208378 & 0.0000000000000000\end{array}$

$\begin{array}{llll}0.0000000000000000 & 0.0000000000000000 & 0.0000000000000000\end{array}$

$\begin{array}{llll}0.5999680613222580 & 0.6666410571604189 & 0.0000000000000000\end{array}$

$\begin{array}{llll}0.7999840306611290 & 0.6666410571604189 & 0.0000000000000000\end{array}$

$\begin{array}{llll}0.7000159693388710 & 0.8332821143208378 & 0.0000000000000000\end{array}$

$\begin{array}{llll}0.3999520919833941 & 0.3332821143208378 & 0.0000000000000000\end{array}$

$\begin{array}{llll}0.5999680613222580 & 0.3332821143208378 & 0.0000000000000000\end{array}$

$\begin{array}{llll}0.5000000000000000 & 0.4999231714812566 & 0.0000000000000000\end{array}$

$\begin{array}{llll}0.5000000000000000 & 0.8332821143208378 & 0.0000000000000000\end{array}$

$\begin{array}{llll}0.2000159693388710 & 0.0000000000000000 & 0.0000000000000000\end{array}$

$\begin{array}{lllll}0.3999520919833941 & 0.0000000000000000 & 0.0000000000000000\end{array}$

$\begin{array}{lll}0.2999840306611290 & 0.1666410571604189 & 0.0000000000000000\end{array}$

$\begin{array}{lll}0.2999840306611290 & 0.4999231714812566 & 0.0000000000000000\end{array}$

$\begin{array}{llll}0.0999680613222580 & 0.1666410571604189 & 0.0000000000000000\end{array}$

$\begin{array}{llll}0.7999840306611290 & 0.3332821143208378 & 0.0000000000000000\end{array}$

$\begin{array}{llll}0.8999520919833941 & 0.4999231714812566 & 0.0000000000000000\end{array}$

$\begin{array}{llll}0.8999520919833941 & 0.8332821143208378 & 0.0000000000000000\end{array}$

$\begin{array}{llll}0.5999680613222580 & 0.0000000000000000 & 0.0000000000000000\end{array}$

$\begin{array}{llll}0.7999840306611290 & 0.0000000000000000 & 0.0000000000000000\end{array}$

$\begin{array}{llll}0.7000159693388710 & 0.1666410571604189 & 0.0000000000000000\end{array}$

$\begin{array}{llll}0.7000159693388710 & 0.4999231714812566 & 0.0000000000000000\end{array}$

$\begin{array}{llll}0.5000000000000000 & 0.1666410571604189 & 0.0000000000000000\end{array}$

$\begin{array}{llll}0.8999520919833941 & 0.1666410571604189 & 0.0000000000000000\end{array}$

$\begin{array}{lllll}0.0000000000000000 & 0.8888291333743084 & 0.0511249999999990\end{array}$

$\begin{array}{llll}0.2000159693388710 & 0.8888291333743084 & 0.0511249999999990\end{array}$

$\begin{array}{llll}0.3999520919833941 & 0.8888291333743084 & 0.0511249999999990\end{array}$

$\begin{array}{llll}0.0000000000000000 & 0.5554701905347272 & 0.0511249999999990\end{array}$

$\begin{array}{llll}0.2000159693388710 & 0.5554701905347272 & 0.0511249999999990\end{array}$

$\begin{array}{llll}0.0999680613222580 & 0.7221880762138895 & 0.0511249999999990\end{array}$

$\begin{array}{llll}0.0000000000000000 & 0.2221880762138895 & 0.0511249999999990\end{array}$

$\begin{array}{llll}0.5999680613222580 & 0.8888291333743084 & 0.0511249999999990\end{array}$

$\begin{array}{llll}0.7999840306611290 & 0.8888291333743084 & 0.0511249999999990\end{array}$

$\begin{array}{llll}0.3999520919833941 & 0.5554701905347272 & 0.0511249999999990\end{array}$

$\begin{array}{llll}0.5999680613222580 & 0.5554701905347272 & 0.0511249999999990\end{array}$

$\begin{array}{llll}0.5000000000000000 & 0.7221880762138895 & 0.0511249999999990\end{array}$

$\begin{array}{llll}0.2000159693388710 & 0.2221880762138895 & 0.0511249999999990\end{array}$ 
$\begin{array}{lll}0.3999520919833941 & 0.2221880762138895 & 0.0511249999999990\end{array}$ $\begin{array}{llll}0.2999840306611290 & 0.3888291333743084 & 0.0511249999999990\end{array}$ $\begin{array}{llll}0.2999840306611290 & 0.7221880762138895 & 0.0511249999999990\end{array}$ $\begin{array}{llll}0.0999680613222580 & 0.0555470190534706 & 0.0511249999999990\end{array}$ $\begin{array}{llll}0.0999680613222580 & 0.3888291333743084 & 0.0511249999999990\end{array}$ $\begin{array}{llll}0.7999840306611290 & 0.5554701905347272 & 0.0511249999999990\end{array}$ $\begin{array}{llll}0.8999520919833941 & 0.7221880762138895 & 0.0511249999999990\end{array}$ $\begin{array}{llll}0.5999680613222580 & 0.2221880762138895 & 0.0511249999999990\end{array}$ $\begin{array}{llll}0.7999840306611290 & 0.2221880762138895 & 0.0511249999999990\end{array}$ $\begin{array}{llll}0.7000159693388710 & 0.3888291333743084 & 0.0511249999999990\end{array}$ $\begin{array}{llll}0.7000159693388710 & 0.7221880762138895 & 0.0511249999999990\end{array}$ $\begin{array}{llll}0.5000000000000000 & 0.0555470190534706 & 0.0511249999999990\end{array}$ $\begin{array}{llll}0.5000000000000000 & 0.3888291333743084 & 0.0511249999999990\end{array}$ $\begin{array}{llll}0.2999840306611290 & 0.0555470190534706 & 0.0511249999999990\end{array}$ $\begin{array}{llll}0.8999520919833941 & 0.0555470190534706 & 0.0511249999999990\end{array}$ $\begin{array}{llll}0.8999520919833941 & 0.3888291333743084 & 0.0511249999999990\end{array}$ $\begin{array}{llll}0.7000159693388710 & 0.0555470190534706 & 0.0511249999999990\end{array}$ $\begin{array}{llll}0.4990834678766238 & 0.6116677657967771 & 0.1015107670815058\end{array}$ $\begin{array}{llll}0.4007744452293227 & 0.7784843005621861 & 0.1029254455552404\end{array}$ $\begin{array}{llll}0.5990110237240833 & 0.7791627298162105 & 0.1023404764199586\end{array}$ $\begin{array}{llll}0.7009019570578259 & 0.6109712805602407 & 0.1014605463571102\end{array}$ $\begin{array}{llll}0.2998476875394533 & 0.6102209349839425 & 0.1028722629830580\end{array}$ $\begin{array}{llll}0.2003726194457581 & 0.4445403074277831 & 0.1027659186249555\end{array}$ $\begin{array}{llll}0.8010204276947848 & 0.4438016027195141 & 0.1019370062570280\end{array}$ $\begin{array}{llll}0.3983512048744824 & 0.4444314025513489 & 0.1025381628223341\end{array}$ $\begin{array}{llll}0.6004338790121400 & 0.4435988299272410 & 0.1014090031205424\end{array}$ $\begin{array}{llll}0.9003600689121017 & 0.2772923207222818 & 0.1022497692035515\end{array}$ $\begin{array}{llll}0.0999930628573272 & 0.2775347091967650 & 0.1022248523451803\end{array}$ $\begin{array}{llll}0.4999915346921964 & 0.9440122693624001 & 0.1028348983471201\end{array}$ $\begin{array}{llll}0.8004329052421472 & 0.1109145691254398 & 0.1022531112100126\end{array}$ $\begin{array}{lllll}0.1998526156095191 & 0.1108472264365981 & 0.1022778675833154\end{array}$ $\begin{array}{llll}0.0000584123607111 & 0.1107881663733302 & 0.1022712249164071\end{array}$ $\begin{array}{llll}0.7001699741529538 & 0.9448897974485381 & 0.1021112056395563\end{array}$ $\begin{array}{llll}0.3001523334167022 & 0.9446033159138704 & 0.1022503660039489\end{array}$ $\begin{array}{llll}0.0999510311118375 & 0.9441793140891239 & 0.1022619160756522\end{array}$ $\begin{array}{llll}0.9003992151071479 & 0.9442724422518970 & 0.1022386678756686\end{array}$ $\begin{array}{llll}0.6000761325198826 & 0.1110861326050234 & 0.1021623319523064\end{array}$ $\begin{array}{llll}0.4000406379638863 & 0.1108852719916248 & 0.1022170915601026\end{array}$ $\begin{array}{llll}0.2999499185186069 & 0.2775157906036780 & 0.1023544008530381\end{array}$ $\begin{array}{llll}0.7008059623404636 & 0.2770407423112397 & 0.1019928940826390\end{array}$ $\begin{array}{llll}0.8006145299782642 & 0.7778176752923486 & 0.1018268201457493\end{array}$ $\begin{array}{llll}1.0002718909862245 & 0.4440201842884768 & 0.1022135438824196\end{array}$ $0.2001466623373495 \quad 0.77755315087214310 .1021588350894838$ $\begin{array}{llll}1.0002881524202474 & 0.7775902174376617 & 0.1022253017075166\end{array}$ $\begin{array}{llll}0.4996615921380335 & 0.2774724709692022 & 0.1019373062082410\end{array}$ $\begin{array}{llll}0.9008409857522632 & 0.6107734240744612 & 0.1020856508738715\end{array}$ $\begin{array}{llll}0.0999484889907569 & 0.6108184863182670 & 0.1022289094656111\end{array}$ 
$\begin{array}{llll}0.6020927318184343 & 0.6659192150729557 & 0.1510338116038988\end{array}$ $\begin{array}{llll}0.3996143242346149 & 0.6669533828112852 & 0.1531465486185165\end{array}$ $\begin{array}{llll}0.2954039851772098 & 0.4967364256659377 & 0.1564246572233733\end{array}$ 0.70172854272612040 .49903809939871540 .1528186116639296 $\begin{array}{llll}0.5025405640037012 & 0.4995199444231657 & 0.1510707339965447\end{array}$ $\begin{array}{llll}0.5019296526024253 & 0.8389907005791198 & 0.1566275931062762\end{array}$ $\begin{array}{llll}0.0994849062252163 & 0.1663340328390496 & 0.1544431566216032\end{array}$ $\begin{array}{llll}0.9001473221912073 & 0.1666974848922358 & 0.1544786383815664\end{array}$ 0.19937787629870490 .00043154501642940 .1544262793426933 $\begin{array}{llll}0.8008766544611791 & 0.0004458705259990 & 0.1545319389001047\end{array}$ $\begin{array}{llll}-0.0000175240807141 & 0.0000739592846220 & 0.1544229441225879\end{array}$ $\begin{array}{llll}-0.0005029707089353 & 0.3331877842933598 & 0.1543836503833164\end{array}$ $\begin{array}{llll}0.7024813437217696 & 0.8345334633665440 & 0.1529818223583601\end{array}$ $\begin{array}{llll}0.2981237515338029 & 0.8346299370566312 & 0.1538122183448065\end{array}$ $\begin{array}{llll}0.6019728758654812 & 0.0022972656785872 & 0.1542195156158556\end{array}$ $\begin{array}{llll}0.3989708864862501 & 0.0017209094032584 & 0.1542645871149511\end{array}$ $\begin{array}{llll}0.2998792106313745 & 0.1667606274217632 & 0.1545635660929667\end{array}$ $\begin{array}{llll}0.7006565305158526 & 0.1670988542094582 & 0.1545334696198961\end{array}$ $\begin{array}{llll}0.1988636236225443 & 0.3314432573567218 & 0.1542641219903744\end{array}$ $\begin{array}{llll}0.8003859708267974 & 0.3333255099822500 & 0.1545705310074050\end{array}$ $\begin{array}{llll}0.9006915362138663 & 0.8335942663776623 & 0.1544114761762728\end{array}$ $\begin{array}{llll}0.0993201610316297 & 0.8337230761122533 & 0.1546042699008853\end{array}$ $\begin{array}{llll}0.5003763304846499 & 0.1665102088924473 & 0.1541850555434433\end{array}$ $\begin{array}{llll}0.4012102360628024 & 0.3317654183173522 & 0.1539089448801869\end{array}$ $\begin{array}{llll}0.6016398285321657 & 0.3320472693998709 & 0.1528503163555180\end{array}$ $\begin{array}{llll}0.0972808307121356 & 0.5000784035093000 & 0.1542464166624750\end{array}$ $\begin{array}{llll}0.8015208960036553 & 0.6668835395490335 & 0.1533235528424593\end{array}$ $\begin{array}{llll}0.8994380405424933 & 0.5001887566088602 & 0.1545193795102395\end{array}$ $\begin{array}{llll}0.1975126603971425 & 0.6677722639531377 & 0.1538226066809581\end{array}$ $\begin{array}{llll}0.9993437059493613 & 0.6669588353798640 & 0.1546316194401912\end{array}$ $\begin{array}{llll}0.3028035099494068 & 0.5051935901574909 & 0.2086680153953756\end{array}$ $\begin{array}{lllll}0.5048786923505109 & 0.8231663806282599 & 0.2083843353615752\end{array}$ $\begin{array}{llll}0.4837916226662097 & 0.3934406241955452 & 0.2145841093995922\end{array}$ $\begin{array}{llll}0.6902698568149341 & 0.7158202108394213 & 0.2158295284778358\end{array}$ $\begin{array}{llll}0.3987129560111521 & 0.6670881965263905 & 0.2098400964369720\end{array}$ $\begin{array}{llll}0.4924188997968614 & 0.5001819013401736 & 0.2149200724778821\end{array}$ $\begin{array}{llll}0.3928244155415866 & 0.5567386535458485 & 0.2114135099739303\end{array}$ 0.59512531837856550 .66160308274836030 .2151678254137916 $\begin{array}{lllll}0.5909047194122748 & 0.5527984287823281 & 0.2167299910296861\end{array}$ $\begin{array}{llll}0.4981127274523812 & 0.7222259637678887 & 0.2113121435787962\end{array}$ $\begin{array}{llll}0.3245748333186153 & 0.7107281672310186 & 0.2123488905194356\end{array}$ $\begin{array}{llll}0.6651464962307294 & 0.5093113009148251 & 0.2191136129798507\end{array}$ $\begin{array}{llll}0.6845855311034605 & 0.7929436445529776 & 0.2191548366641942\end{array}$ $\begin{array}{lllll}0.7602244044664953 & 0.6807362380623704 & 0.2212031618540269\end{array}$ $\begin{array}{llll}0.5479312845532371 & 0.3495712433598927 & 0.2203528320248061\end{array}$ $\begin{array}{llll}0.4104477461615645 & 0.3651771026274553 & 0.2204213794011382\end{array}$ 
$\underline{\text { Zwitterion on } \mathrm{Cu} \text { - VASP - revPBE }+\mathrm{D} 3-\mathrm{calculated} \text { lattice }-\left(\mathrm{H}_{\mathrm{fcc}}-0 \text { site }\right)}$

$\mathrm{Cu} \mathrm{O} \mathrm{N} \mathrm{C} \mathrm{H}$

1.00000000000000

$\begin{array}{lll}12.5239999999999991 & 0.0000000000000000 & 0.0000000000000000\end{array}$

$\begin{array}{lll}0.0000000000000000 & 13.0160000000000000 & 0.0000000000000000\end{array}$

$\begin{array}{llll}0.0000000000000000 & 0.0000000000000000 & 40.0000000000000000\end{array}$

$\mathrm{Cu} \quad \mathrm{O} \quad \mathrm{N} \quad \mathrm{C} \quad \mathrm{H}$

$\begin{array}{lllll}120 & 2 & 2 & 6 & 6\end{array}$

Direct

$0.0000000000000000 \quad 0.66664105716041890 .0000000000000000$

$\begin{array}{llll}0.2000159693388710 & 0.6666410571604189 & 0.0000000000000000\end{array}$

0.39995209198339410 .66664105716041890 .0000000000000000

$\begin{array}{llll}0.2999840306611290 & 0.8332821143208378 & 0.0000000000000000\end{array}$

$\begin{array}{llll}0.0000000000000000 & 0.3332821143208378 & 0.0000000000000000\end{array}$

$\begin{array}{llll}0.2000159693388710 & 0.3332821143208378 & 0.0000000000000000\end{array}$

$\begin{array}{llll}0.0999680613222580 & 0.4999231714812566 & 0.0000000000000000\end{array}$

$\begin{array}{llll}0.0999680613222580 & 0.8332821143208378 & 0.0000000000000000\end{array}$

$\begin{array}{llll}0.0000000000000000 & 0.0000000000000000 & 0.0000000000000000\end{array}$

$\begin{array}{lll}0.5999680613222580 & 0.6666410571604189 & 0.0000000000000000\end{array}$

$\begin{array}{llll}0.7999840306611290 & 0.6666410571604189 & 0.0000000000000000\end{array}$

$\begin{array}{llll}0.7000159693388710 & 0.8332821143208378 & 0.0000000000000000\end{array}$

$\begin{array}{llll}0.3999520919833941 & 0.3332821143208378 & 0.0000000000000000\end{array}$

$\begin{array}{llll}0.5999680613222580 & 0.3332821143208378 & 0.0000000000000000\end{array}$

$\begin{array}{llll}0.5000000000000000 & 0.4999231714812566 & 0.0000000000000000\end{array}$

$\begin{array}{llll}0.5000000000000000 & 0.8332821143208378 & 0.0000000000000000\end{array}$

$\begin{array}{llll}0.2000159693388710 & 0.0000000000000000 & 0.0000000000000000\end{array}$

$\begin{array}{llll}0.3999520919833941 & 0.0000000000000000 & 0.0000000000000000\end{array}$

$\begin{array}{lll}0.2999840306611290 & 0.1666410571604189 & 0.0000000000000000\end{array}$

$\begin{array}{llll}0.2999840306611290 & 0.4999231714812566 & 0.0000000000000000\end{array}$

$\begin{array}{llll}0.0999680613222580 & 0.1666410571604189 & 0.0000000000000000\end{array}$

$\begin{array}{llll}0.7999840306611290 & 0.3332821143208378 & 0.0000000000000000\end{array}$

$\begin{array}{llll}0.8999520919833941 & 0.4999231714812566 & 0.0000000000000000\end{array}$

$\begin{array}{llll}0.8999520919833941 & 0.8332821143208378 & 0.0000000000000000\end{array}$

$\begin{array}{llll}0.5999680613222580 & 0.0000000000000000 & 0.0000000000000000\end{array}$

$\begin{array}{llll}0.7999840306611290 & 0.0000000000000000 & 0.0000000000000000\end{array}$

$\begin{array}{llll}0.7000159693388710 & 0.1666410571604189 & 0.0000000000000000\end{array}$

$\begin{array}{llll}0.7000159693388710 & 0.4999231714812566 & 0.0000000000000000\end{array}$

$\begin{array}{llll}0.5000000000000000 & 0.1666410571604189 & 0.0000000000000000\end{array}$

$\begin{array}{llll}0.8999520919833941 & 0.1666410571604189 & 0.0000000000000000\end{array}$

$\begin{array}{llll}0.0000000000000000 & 0.8888291333743084 & 0.0511249999999990\end{array}$

$\begin{array}{llll}0.2000159693388710 & 0.8888291333743084 & 0.0511249999999990\end{array}$

$\begin{array}{llll}0.3999520919833941 & 0.8888291333743084 & 0.0511249999999990\end{array}$

$\begin{array}{llll}0.0000000000000000 & 0.5554701905347272 & 0.0511249999999990\end{array}$

$\begin{array}{llll}0.2000159693388710 & 0.5554701905347272 & 0.0511249999999990\end{array}$

$\begin{array}{llll}0.0999680613222580 & 0.7221880762138895 & 0.0511249999999990\end{array}$

$\begin{array}{lllll}0.0000000000000000 & 0.2221880762138895 & 0.0511249999999990\end{array}$

$\begin{array}{llll}0.5999680613222580 & 0.8888291333743084 & 0.0511249999999990\end{array}$ 
$\begin{array}{llll}0.7999840306611290 & 0.8888291333743084 & 0.0511249999999990\end{array}$

$\begin{array}{llll}0.3999520919833941 & 0.5554701905347272 & 0.0511249999999990\end{array}$

$\begin{array}{llll}0.5999680613222580 & 0.5554701905347272 & 0.0511249999999990\end{array}$

$\begin{array}{llll}0.5000000000000000 & 0.7221880762138895 & 0.0511249999999990\end{array}$

$\begin{array}{llll}0.2000159693388710 & 0.2221880762138895 & 0.0511249999999990\end{array}$

$\begin{array}{llll}0.3999520919833941 & 0.2221880762138895 & 0.0511249999999990\end{array}$

$\begin{array}{llll}0.2999840306611290 & 0.3888291333743084 & 0.0511249999999990\end{array}$

$\begin{array}{llll}0.2999840306611290 & 0.7221880762138895 & 0.0511249999999990\end{array}$

$\begin{array}{llll}0.0999680613222580 & 0.0555470190534706 & 0.0511249999999990\end{array}$

$\begin{array}{llll}0.0999680613222580 & 0.3888291333743084 & 0.0511249999999990\end{array}$

$\begin{array}{llll}0.7999840306611290 & 0.5554701905347272 & 0.0511249999999990\end{array}$

$\begin{array}{llll}0.8999520919833941 & 0.7221880762138895 & 0.0511249999999990\end{array}$

$\begin{array}{llll}0.5999680613222580 & 0.2221880762138895 & 0.0511249999999990\end{array}$

$\begin{array}{llll}0.7999840306611290 & 0.2221880762138895 & 0.0511249999999990\end{array}$

$\begin{array}{llll}0.7000159693388710 & 0.3888291333743084 & 0.0511249999999990\end{array}$

$\begin{array}{llll}0.7000159693388710 & 0.7221880762138895 & 0.0511249999999990\end{array}$

$\begin{array}{llll}0.5000000000000000 & 0.0555470190534706 & 0.0511249999999990\end{array}$

$\begin{array}{llll}0.5000000000000000 & 0.3888291333743084 & 0.0511249999999990\end{array}$

$\begin{array}{llll}0.2999840306611290 & 0.0555470190534706 & 0.0511249999999990\end{array}$

$\begin{array}{llll}0.8999520919833941 & 0.0555470190534706 & 0.0511249999999990\end{array}$

$\begin{array}{llll}0.8999520919833941 & 0.3888291333743084 & 0.0511249999999990\end{array}$

$\begin{array}{llll}0.7000159693388710 & 0.0555470190534706 & 0.0511249999999990\end{array}$

$\begin{array}{llll}0.5008034405885698 & 0.6119284000951503 & 0.1026407328844104\end{array}$

$\begin{array}{llll}0.4000345741198986 & 0.7780853458365337 & 0.1021477327547329\end{array}$

$\begin{array}{llll}0.6000712818088013 & 0.7771646708122494 & 0.1029333272203092\end{array}$

$\begin{array}{llll}0.6995413781329480 & 0.6124246471182814 & 0.1023385171220990\end{array}$

$\begin{array}{llll}0.2994021477457140 & 0.6109611468374334 & 0.1022636075540678\end{array}$

$\begin{array}{llll}0.1994620053768484 & 0.4437757182318474 & 0.1027533095686981\end{array}$

$\begin{array}{llll}0.8003951697130659 & 0.4441433022186640 & 0.1022659073507649\end{array}$

$\begin{array}{llll}0.3988738916031352 & 0.4456105525100020 & 0.1014166501573419\end{array}$

$\begin{array}{llll}0.5997685585564014 & 0.4449660623781488 & 0.1021847887567521\end{array}$

$\begin{array}{llll}0.8997932476917551 & 0.2781460414235968 & 0.1026908324894492\end{array}$

$\begin{array}{llll}0.1005642188070611 & 0.2781913909382737 & 0.1029884979235632\end{array}$

$\begin{array}{llll}0.5003410430728551 & 0.9440504316728652 & 0.1021947639124786\end{array}$

$\begin{array}{llll}0.8010078385828097 & 0.1109166498581630 & 0.1021755719439863\end{array}$

$\begin{array}{llll}0.1999684282642303 & 0.1111135674647300 & 0.1025941202517992\end{array}$

$\begin{array}{llll}0.0003979656498780 & 0.1114136219053542 & 0.1022364705442224\end{array}$

$\begin{array}{llll}0.7004122947771599 & 0.9442035567960515 & 0.1022202499087256\end{array}$

$\begin{array}{llll}0.2998680420812861 & 0.9443318402202551 & 0.1022755197905440\end{array}$

$\begin{array}{llll}0.1000386821505411 & 0.9445136953572112 & 0.1022453723790004\end{array}$

$\begin{array}{llll}0.9004392006734039 & 0.9445623920228955 & 0.1022772066136465\end{array}$

$\begin{array}{llll}0.6012414092066451 & 0.1101819407971692 & 0.1016564122049835\end{array}$

$\begin{array}{llll}0.3991990265305150 & 0.1104202329593384 & 0.1019176531567697\end{array}$

$\begin{array}{llll}0.2980607108337069 & 0.2771325454434111 & 0.1021477052032755\end{array}$

$\begin{array}{llll}0.7014380328795012 & 0.2778955588479948 & 0.1023604443562747\end{array}$

$\begin{array}{llll}0.8001104545752760 & 0.7780942894816761 & 0.1022937825441359\end{array}$

$\begin{array}{llll}0.9999999964688976 & 0.4440077617240843 & 0.1022436538288408\end{array}$ 
$\begin{array}{lll}0.1997964928848295 & 0.7779182697935283 & 0.1022190189291787\end{array}$ $\begin{array}{llll}1.0000676029632285 & 0.7778153119678446 & 0.1022417550947536\end{array}$ $\begin{array}{llll}0.5004322109888186 & 0.2771720922801263 & 0.1010263702956113\end{array}$ $\begin{array}{llll}0.9000275997218125 & 0.6112042221073030 & 0.1021330061486447\end{array}$ 0.09964924886510130 .61105064179601040 .1021813924764299 $\begin{array}{llll}0.5997702584606345 & 0.6672715555519518 & 0.1571510482289347\end{array}$ $\begin{array}{llll}0.3984591471242018 & 0.6683521832390374 & 0.1535422033788731\end{array}$ $\begin{array}{llll}0.2955045084693710 & 0.5021445605144358 & 0.1541903376300208\end{array}$ $\begin{array}{llll}0.7005919956200997 & 0.5028054277880702 & 0.1522939593186780\end{array}$ $\begin{array}{llll}0.4950809232788672 & 0.4997615315652232 & 0.1526690296432790\end{array}$ $\begin{array}{llll}0.4999265489273820 & 0.8334550102751563 & 0.1545008730361342\end{array}$ $\begin{array}{llll}0.1001872578947767 & 0.1669002791274601 & 0.1547635306001768\end{array}$ $\begin{array}{llll}0.9001315907380860 & 0.1671616807339890 & 0.1545507893953011\end{array}$ $\begin{array}{llll}0.2000082934681170 & 0.0000621701688509 & 0.1546483411611009\end{array}$ $\begin{array}{llll}0.8005111326237251 & 0.0001026733286802 & 0.1544418671421341\end{array}$ $\begin{array}{llll}0.0002151224737689 & 0.0005684739696419 & 0.1544223366272360\end{array}$ $\begin{array}{llll}-0.0003668807466838 & 0.3336743497410505 & 0.1547742688074839\end{array}$ 0.70039358969380090 .83377814206415560 .1544413260115662 $0.29972169436297490 .8338524344976297 \quad 0.1544390999556687$ $0.6004036465422314-0.00046057272292410 .1544564526273644$ $0.4004094372627378-0.00083223099831860 .1542628760210426$ $\begin{array}{llll}0.3013120925041954 & 0.1655809485819590 & 0.1548983558511186\end{array}$ $\begin{array}{llll}0.7011234511119911 & 0.1654260312596031 & 0.1538019175127294\end{array}$ $\begin{array}{llll}0.2002089787118316 & 0.3333209892801198 & 0.1571157168421866\end{array}$ $\begin{array}{llll}0.7992986354973324 & 0.3349593913035374 & 0.1562142371985964\end{array}$ $\begin{array}{llll}0.9004515680812430 & 0.8339694743157491 & 0.1544259748740868\end{array}$ $\begin{array}{lllll}0.0996833261547171 & 0.8339546561308641 & 0.1543567101962486\end{array}$ $\begin{array}{lll}0.5018994748170235 & 0.1636366932431069 & 0.1516936692475300\end{array}$ $\begin{array}{llll}0.3998621910031332 & 0.3321622815066891 & 0.1498862923678715\end{array}$ $\begin{array}{llll}0.5984567754853636 & 0.3308676328628809 & 0.1520476405924352\end{array}$ $\begin{array}{llll}0.0975674651805549 & 0.5007983679287082 & 0.1543292556010817\end{array}$ $\begin{array}{llll}0.8013900435637338 & 0.6677959925661097 & 0.1541374957531236\end{array}$ $\begin{array}{llll}0.9003857369640696 & 0.5006724237497199 & 0.1541368882817675\end{array}$ $\begin{array}{llll}0.1988061882845174 & 0.6679116467172737 & 0.1542609639535288\end{array}$ $\begin{array}{llll}0.9999849005394189 & 0.6673749297143829 & 0.1544954853549412\end{array}$ $\begin{array}{llll}0.6962335067192832 & 0.4941145562238012 & 0.2096115096547892\end{array}$ $\begin{array}{llll}0.3073888721123912 & 0.4920791825587821 & 0.2083017417295731\end{array}$ $\begin{array}{llll}0.7004785932857180 & 0.2827522322896754 & 0.2124698568089392\end{array}$ $\begin{array}{llll}0.3051339621213989 & 0.2822525027589748 & 0.2134681331756759\end{array}$ $\begin{array}{llll}0.6037075437239128 & 0.4464869186598341 & 0.2113052595120859\end{array}$ $\begin{array}{llll}0.5027455699235135 & 0.2810575416775648 & 0.2152109977585783\end{array}$ $\begin{array}{llll}0.6008432975908239 & 0.3343429580973831 & 0.2137160603575920\end{array}$ $\begin{array}{llll}0.3999292020849012 & 0.4444849464590748 & 0.2112524974882019\end{array}$ $\begin{array}{llll}0.4032623336908513 & 0.3336270297382975 & 0.2144677235981623\end{array}$ $\begin{array}{llll}0.5015816818958809 & 0.4975316393574839 & 0.2098530700751097\end{array}$ $\begin{array}{lllll}0.2409622491958301 & 0.3256012431866874 & 0.2201129722985411\end{array}$ $\begin{array}{llll}0.3024426390023908 & 0.2066051414090126 & 0.2193960703947282\end{array}$ 
$\begin{array}{llll}0.7051876834028392 & 0.2134792782089237 & 0.2240075857017593\end{array}$

$\begin{array}{llll}0.7626745646594831 & 0.3309740069567483 & 0.2176156620863921\end{array}$

$\begin{array}{llll}0.5036293147049014 & 0.1971447012587504 & 0.2165383644019275\end{array}$

$\begin{array}{llll}0.4998364364869048 & 0.5811413383372381 & 0.2123292500323512\end{array}$

$\underline{\text { Zwitterion on } \mathrm{Cu}-\mathrm{VASP} \text { - revPBE}+\mathrm{D} 3 \text {-calculated lattice }-\left(\mathrm{H}_{\mathrm{fcc}}-30 \text { site }\right)}$

$\mathrm{Cu} \mathrm{O} \mathrm{N} \mathrm{C} \mathrm{H}$

1.00000000000000

$\begin{array}{lll}12.5239999999999991 & 0.0000000000000000 & 0.0000000000000000\end{array}$

$\begin{array}{lll}0.0000000000000000 & 13.0160000000000000 & 0.0000000000000000\end{array}$

$\begin{array}{llll}0.0000000000000000 & 0.0000000000000000 & 40.0000000000000000\end{array}$

$\begin{array}{llllll}\mathrm{Cu} & \mathrm{O} & \mathrm{N} & \mathrm{C} & \mathrm{H}\end{array}$

$\begin{array}{lllll}120 & 2 & 2 & 6 & 6\end{array}$

Direct

$\begin{array}{llll}0.0000000000000000 & 0.6666410571604189 & 0.0000000000000000\end{array}$

$\begin{array}{llll}0.2000159693388710 & 0.6666410571604189 & 0.0000000000000000\end{array}$

0.39995209198339410 .66664105716041890 .0000000000000000

$\begin{array}{llll}0.2999840306611290 & 0.8332821143208378 & 0.0000000000000000\end{array}$

$\begin{array}{llll}0.0000000000000000 & 0.3332821143208378 & 0.0000000000000000\end{array}$

$\begin{array}{llll}0.2000159693388710 & 0.3332821143208378 & 0.0000000000000000\end{array}$

$\begin{array}{llll}0.0999680613222580 & 0.4999231714812566 & 0.0000000000000000\end{array}$

$\begin{array}{llll}0.0999680613222580 & 0.8332821143208378 & 0.0000000000000000\end{array}$

$\begin{array}{lll}0.0000000000000000 & 0.0000000000000000 & 0.0000000000000000\end{array}$

$\begin{array}{lll}0.5999680613222580 & 0.6666410571604189 & 0.0000000000000000\end{array}$

$\begin{array}{lll}0.7999840306611290 & 0.6666410571604189 & 0.0000000000000000\end{array}$

$\begin{array}{llll}0.7000159693388710 & 0.8332821143208378 & 0.0000000000000000\end{array}$

$\begin{array}{llll}0.3999520919833941 & 0.3332821143208378 & 0.0000000000000000\end{array}$

$\begin{array}{llll}0.5999680613222580 & 0.3332821143208378 & 0.0000000000000000\end{array}$

$\begin{array}{llll}0.5000000000000000 & 0.4999231714812566 & 0.0000000000000000\end{array}$

$\begin{array}{llll}0.5000000000000000 & 0.8332821143208378 & 0.0000000000000000\end{array}$

$\begin{array}{llll}0.2000159693388710 & 0.0000000000000000 & 0.0000000000000000\end{array}$

$\begin{array}{llll}0.3999520919833941 & 0.0000000000000000 & 0.0000000000000000\end{array}$

$\begin{array}{lll}0.2999840306611290 & 0.1666410571604189 & 0.0000000000000000\end{array}$

$\begin{array}{lll}0.2999840306611290 & 0.4999231714812566 & 0.0000000000000000\end{array}$

$\begin{array}{llll}0.0999680613222580 & 0.1666410571604189 & 0.0000000000000000\end{array}$

$\begin{array}{llll}0.7999840306611290 & 0.3332821143208378 & 0.0000000000000000\end{array}$

$\begin{array}{llll}0.8999520919833941 & 0.4999231714812566 & 0.0000000000000000\end{array}$

$\begin{array}{llll}0.8999520919833941 & 0.8332821143208378 & 0.0000000000000000\end{array}$

$\begin{array}{llll}0.5999680613222580 & 0.0000000000000000 & 0.0000000000000000\end{array}$

$\begin{array}{llll}0.7999840306611290 & 0.0000000000000000 & 0.0000000000000000\end{array}$

$\begin{array}{llll}0.7000159693388710 & 0.1666410571604189 & 0.0000000000000000\end{array}$

$\begin{array}{lll}0.7000159693388710 & 0.4999231714812566 & 0.0000000000000000\end{array}$

$\begin{array}{llll}0.5000000000000000 & 0.1666410571604189 & 0.0000000000000000\end{array}$

$\begin{array}{llll}0.8999520919833941 & 0.1666410571604189 & 0.0000000000000000\end{array}$

$\begin{array}{llll}0.0000000000000000 & 0.8888291333743084 & 0.0511249999999990\end{array}$

$\begin{array}{llll}0.2000159693388710 & 0.8888291333743084 & 0.0511249999999990\end{array}$

$\begin{array}{llll}0.3999520919833941 & 0.8888291333743084 & 0.0511249999999990\end{array}$ 
$\begin{array}{llll}0.0000000000000000 & 0.5554701905347272 & 0.0511249999999990\end{array}$

$\begin{array}{llll}0.2000159693388710 & 0.5554701905347272 & 0.0511249999999990\end{array}$

$\begin{array}{llll}0.0999680613222580 & 0.7221880762138895 & 0.0511249999999990\end{array}$

$\begin{array}{llll}0.0000000000000000 & 0.2221880762138895 & 0.0511249999999990\end{array}$

$\begin{array}{llll}0.5999680613222580 & 0.8888291333743084 & 0.0511249999999990\end{array}$

$\begin{array}{llll}0.7999840306611290 & 0.8888291333743084 & 0.0511249999999990\end{array}$

$\begin{array}{llll}0.3999520919833941 & 0.5554701905347272 & 0.0511249999999990\end{array}$

$\begin{array}{llll}0.5999680613222580 & 0.5554701905347272 & 0.0511249999999990\end{array}$

$\begin{array}{llll}0.5000000000000000 & 0.7221880762138895 & 0.0511249999999990\end{array}$

$\begin{array}{llll}0.2000159693388710 & 0.2221880762138895 & 0.0511249999999990\end{array}$

$\begin{array}{llll}0.3999520919833941 & 0.2221880762138895 & 0.0511249999999990\end{array}$

$\begin{array}{llll}0.2999840306611290 & 0.3888291333743084 & 0.0511249999999990\end{array}$

$\begin{array}{lll}0.2999840306611290 & 0.7221880762138895 & 0.0511249999999990\end{array}$

$\begin{array}{llll}0.0999680613222580 & 0.0555470190534706 & 0.0511249999999990\end{array}$

$\begin{array}{llll}0.0999680613222580 & 0.3888291333743084 & 0.0511249999999990\end{array}$

$\begin{array}{llll}0.7999840306611290 & 0.5554701905347272 & 0.0511249999999990\end{array}$

$\begin{array}{llll}0.8999520919833941 & 0.7221880762138895 & 0.0511249999999990\end{array}$

$\begin{array}{llll}0.5999680613222580 & 0.2221880762138895 & 0.0511249999999990\end{array}$

$\begin{array}{llll}0.7999840306611290 & 0.2221880762138895 & 0.0511249999999990\end{array}$

$\begin{array}{llll}0.7000159693388710 & 0.3888291333743084 & 0.0511249999999990\end{array}$

$\begin{array}{llll}0.7000159693388710 & 0.7221880762138895 & 0.0511249999999990\end{array}$

$\begin{array}{llll}0.5000000000000000 & 0.0555470190534706 & 0.0511249999999990\end{array}$

$\begin{array}{llll}0.5000000000000000 & 0.3888291333743084 & 0.0511249999999990\end{array}$

$\begin{array}{llll}0.2999840306611290 & 0.0555470190534706 & 0.0511249999999990\end{array}$

$\begin{array}{llll}0.8999520919833941 & 0.0555470190534706 & 0.0511249999999990\end{array}$

$\begin{array}{lllll}0.8999520919833941 & 0.3888291333743084 & 0.0511249999999990\end{array}$

$\begin{array}{llll}0.7000159693388710 & 0.0555470190534706 & 0.0511249999999990\end{array}$

$\begin{array}{llll}0.5008034405885698 & 0.6119284000951503 & 0.1026407328844104\end{array}$

$\begin{array}{llll}0.4000345741198986 & 0.7780853458365337 & 0.1021477327547329\end{array}$

$\begin{array}{llll}0.6000712818088013 & 0.7771646708122494 & 0.1029333272203092\end{array}$

$\begin{array}{llll}0.6995413781329480 & 0.6124246471182814 & 0.1023385171220990\end{array}$

$\begin{array}{llll}0.2994021477457140 & 0.6109611468374334 & 0.1022636075540678\end{array}$

$\begin{array}{lll}0.1994620053768484 & 0.4437757182318474 & 0.1027533095686981\end{array}$

$\begin{array}{llll}0.8003951697130659 & 0.4441433022186640 & 0.1022659073507649\end{array}$

$\begin{array}{llll}0.3988738916031352 & 0.4456105525100020 & 0.1014166501573419\end{array}$

$\begin{array}{llll}0.5997685585564014 & 0.4449660623781488 & 0.1021847887567521\end{array}$

$\begin{array}{llll}0.8997932476917551 & 0.2781460414235968 & 0.1026908324894492\end{array}$

$\begin{array}{llll}0.1005642188070611 & 0.2781913909382737 & 0.1029884979235632\end{array}$

$\begin{array}{llll}0.5003410430728551 & 0.9440504316728652 & 0.1021947639124786\end{array}$

$\begin{array}{llll}0.8010078385828097 & 0.1109166498581630 & 0.1021755719439863\end{array}$

$\begin{array}{llll}0.1999684282642303 & 0.1111135674647300 & 0.1025941202517992\end{array}$

$\begin{array}{llll}0.0003979656498780 & 0.1114136219053542 & 0.1022364705442224\end{array}$

$\begin{array}{llll}0.7004122947771599 & 0.9442035567960515 & 0.1022202499087256\end{array}$

$\begin{array}{llll}0.2998680420812861 & 0.9443318402202551 & 0.1022755197905440\end{array}$

$\begin{array}{llll}0.1000386821505411 & 0.9445136953572112 & 0.1022453723790004\end{array}$

0.90043920067340390 .94456239202289550 .1022772066136465

$\begin{array}{llll}0.6012414092066451 & 0.1101819407971692 & 0.1016564122049835\end{array}$ 
$\begin{array}{lll}0.3991990265305150 & 0.1104202329593384 & 0.1019176531567697\end{array}$

$\begin{array}{llll}0.2980607108337069 & 0.2771325454434111 & 0.1021477052032755\end{array}$

$\begin{array}{llll}0.7014380328795012 & 0.2778955588479948 & 0.1023604443562747\end{array}$

$\begin{array}{llll}0.8001104545752760 & 0.7780942894816761 & 0.1022937825441359\end{array}$

$\begin{array}{llll}0.9999999964688976 & 0.4440077617240843 & 0.1022436538288408\end{array}$

$\begin{array}{llll}0.1997964928848295 & 0.7779182697935283 & 0.1022190189291787\end{array}$

$\begin{array}{llll}1.0000676029632285 & 0.7778153119678446 & 0.1022417550947536\end{array}$

$\begin{array}{llll}0.5004322109888186 & 0.2771720922801263 & 0.1010263702956113\end{array}$

$\begin{array}{llll}0.9000275997218125 & 0.6112042221073030 & 0.1021330061486447\end{array}$

0.09964924886510130 .61105064179601040 .1021813924764299

$\begin{array}{llll}0.5997702584606345 & 0.6672715555519518 & 0.1571510482289347\end{array}$

0.39845914712420180 .66835218323903740 .1535422033788731

$\begin{array}{llll}0.2955045084693710 & 0.5021445605144358 & 0.1541903376300208\end{array}$

$\begin{array}{llll}0.7005919956200997 & 0.5028054277880702 & 0.1522939593186780\end{array}$

$\begin{array}{llll}0.4950809232788672 & 0.4997615315652232 & 0.1526690296432790\end{array}$

$\begin{array}{llll}0.4999265489273820 & 0.8334550102751563 & 0.1545008730361342\end{array}$

$\begin{array}{llll}0.1001872578947767 & 0.1669002791274601 & 0.1547635306001768\end{array}$

$\begin{array}{llll}0.9001315907380860 & 0.1671616807339890 & 0.1545507893953011\end{array}$

$\begin{array}{llll}0.2000082934681170 & 0.0000621701688509 & 0.1546483411611009\end{array}$

$\begin{array}{llll}0.8005111326237251 & 0.0001026733286802 & 0.1544418671421341\end{array}$

0.00021512247376890 .00056847396964190 .1544223366272360

$\begin{array}{llll}-0.0003668807466838 & 0.3336743497410505 & 0.1547742688074839\end{array}$

0.70039358969380090 .83377814206415560 .1544413260115662

$\begin{array}{llll}0.2997216943629749 & 0.8338524344976297 & 0.1544390999556687\end{array}$

$0.6004036465422314-0.00046057272292410 .1544564526273644$

$0.4004094372627378-0.00083223099831860 .1542628760210426$

$\begin{array}{llll}0.3013120925041954 & 0.1655809485819590 & 0.1548983558511186\end{array}$

$\begin{array}{llll}0.7011234511119911 & 0.1654260312596031 & 0.1538019175127294\end{array}$

$\begin{array}{llll}0.2002089787118316 & 0.3333209892801198 & 0.1571157168421866\end{array}$

$\begin{array}{llll}0.7992986354973324 & 0.3349593913035374 & 0.1562142371985964\end{array}$

$\begin{array}{llll}0.9004515680812430 & 0.8339694743157491 & 0.1544259748740868\end{array}$

$\begin{array}{llll}0.0996833261547171 & 0.8339546561308641 & 0.1543567101962486\end{array}$

$\begin{array}{llll}0.5018994748170235 & 0.1636366932431069 & 0.1516936692475300\end{array}$

$\begin{array}{llll}0.3998621910031332 & 0.3321622815066891 & 0.1498862923678715\end{array}$

$\begin{array}{llll}0.5984567754853636 & 0.3308676328628809 & 0.1520476405924352\end{array}$

$\begin{array}{llll}0.0975674651805549 & 0.5007983679287082 & 0.1543292556010817\end{array}$

$\begin{array}{llll}0.8013900435637338 & 0.6677959925661097 & 0.1541374957531236\end{array}$

$\begin{array}{llll}0.9003857369640696 & 0.5006724237497199 & 0.1541368882817675\end{array}$

$0.19880618828451740 .6679116467172737 \quad 0.1542609639535288$

$\begin{array}{llll}0.9999849005394189 & 0.6673749297143829 & 0.1544954853549412\end{array}$

$\begin{array}{llll}0.2830229674425667 & 0.3971712786126880 & 0.2083017417072647\end{array}$

$\begin{array}{llll}0.6285257329686398 & 0.5688437438853878 & 0.2096115096416105\end{array}$

$\begin{array}{llll}0.3800153688253421 & 0.2092293259810950 & 0.2134681331468583\end{array}$

$\begin{array}{llll}0.7320342294879937 & 0.3823729145847554 & 0.2124698567822218\end{array}$

$\begin{array}{llll}0.4534774206380789 & 0.4868586644602267 & 0.2098530700205734\end{array}$

$\begin{array}{llll}0.4432085852241802 & 0.2978697641452649 & 0.2144677234961049\end{array}$

$\begin{array}{llll}0.3879331709590173 & 0.3951888798996800 & 0.2112524973578868\end{array}$ 
$\begin{array}{lll}0.6189167200187655 & 0.3848169190139718 & 0.2137160602798462\end{array}$

$\begin{array}{llll}0.5566524135318777 & 0.2944872520955427 & 0.2152109977068231\end{array}$

$\begin{array}{llll}0.5685564217373764 & 0.4859891077603875 & 0.2113052595337284\end{array}$

$\begin{array}{llll}0.4124732224481065 & 0.5605930764993140 & 0.2123292499542904\end{array}$

$\begin{array}{llll}0.5970320388560157 & 0.2201064098495797 & 0.2165383644195305\end{array}$

$\begin{array}{llll}0.7646989913873068 & 0.4525078858133270 & 0.2176156620218559\end{array}$

$\begin{array}{llll}0.7689147934348255 & 0.3227073316809295 & 0.2240075856880969\end{array}$

$\begin{array}{lllll}0.4133097229367760 & 0.1406513833174781 & 0.2193960702828738\end{array}$

$\begin{array}{llll}0.3023849203937897 & 0.2198212182533612 & 0.2201129721906944\end{array}$

Zwitterion on $\mathrm{Cu}$ - VASP - revPBE+D3-calculated lattice - $\left(\mathrm{H}_{\mathrm{fcc}}-60\right.$ site $)$ $\mathrm{Cu} \mathrm{O} \mathrm{N} \mathrm{C} \mathrm{H}$

1.00000000000000

$\begin{array}{lll}12.5239999999999991 & 0.0000000000000000 & 0.0000000000000000\end{array}$

$\begin{array}{lll}0.0000000000000000 & 13.0160000000000000 & 0.0000000000000000\end{array}$

$\begin{array}{llll}0.0000000000000000 & 0.0000000000000000 & 40.0000000000000000\end{array}$

$\mathrm{Cu} \quad \mathrm{O} \quad \mathrm{N} \quad \mathrm{C} \quad \mathrm{H}$

$\begin{array}{lllll}120 & 2 & 2 & 6 & 6\end{array}$

Direct

$0.0000000000000000 \quad 0.66664105716041890 .0000000000000000$

$\begin{array}{llll}0.2000159693388710 & 0.6666410571604189 & 0.0000000000000000\end{array}$

$\begin{array}{llll}0.3999520919833941 & 0.6666410571604189 & 0.0000000000000000\end{array}$

$\begin{array}{lll}0.2999840306611290 & 0.8332821143208378 & 0.0000000000000000\end{array}$

$\begin{array}{llll}0.0000000000000000 & 0.3332821143208378 & 0.0000000000000000\end{array}$

$\begin{array}{llll}0.2000159693388710 & 0.3332821143208378 & 0.0000000000000000\end{array}$

$\begin{array}{llll}0.0999680613222580 & 0.4999231714812566 & 0.0000000000000000\end{array}$

$\begin{array}{llll}0.0999680613222580 & 0.8332821143208378 & 0.0000000000000000\end{array}$

$\begin{array}{lll}0.0000000000000000 & 0.0000000000000000 & 0.0000000000000000\end{array}$

$\begin{array}{lll}0.5999680613222580 & 0.6666410571604189 & 0.0000000000000000\end{array}$

$\begin{array}{lll}0.7999840306611290 & 0.6666410571604189 & 0.0000000000000000\end{array}$

$\begin{array}{llll}0.7000159693388710 & 0.8332821143208378 & 0.0000000000000000\end{array}$

$\begin{array}{llll}0.3999520919833941 & 0.3332821143208378 & 0.0000000000000000\end{array}$

$\begin{array}{llll}0.5999680613222580 & 0.3332821143208378 & 0.0000000000000000\end{array}$

$\begin{array}{llll}0.5000000000000000 & 0.4999231714812566 & 0.0000000000000000\end{array}$

$\begin{array}{llll}0.5000000000000000 & 0.8332821143208378 & 0.0000000000000000\end{array}$

$\begin{array}{llll}0.2000159693388710 & 0.0000000000000000 & 0.0000000000000000\end{array}$

$\begin{array}{llll}0.3999520919833941 & 0.0000000000000000 & 0.0000000000000000\end{array}$

$\begin{array}{lll}0.2999840306611290 & 0.1666410571604189 & 0.0000000000000000\end{array}$

$\begin{array}{llll}0.2999840306611290 & 0.4999231714812566 & 0.0000000000000000\end{array}$

$\begin{array}{llll}0.0999680613222580 & 0.1666410571604189 & 0.0000000000000000\end{array}$

$\begin{array}{llll}0.7999840306611290 & 0.3332821143208378 & 0.0000000000000000\end{array}$

$\begin{array}{llll}0.8999520919833941 & 0.4999231714812566 & 0.0000000000000000\end{array}$

$\begin{array}{llll}0.8999520919833941 & 0.8332821143208378 & 0.0000000000000000\end{array}$

$\begin{array}{llll}0.5999680613222580 & 0.0000000000000000 & 0.0000000000000000\end{array}$

$\begin{array}{llll}0.7999840306611290 & 0.0000000000000000 & 0.0000000000000000\end{array}$

$\begin{array}{llll}0.7000159693388710 & 0.1666410571604189 & 0.0000000000000000\end{array}$

$\begin{array}{llll}0.7000159693388710 & 0.4999231714812566 & 0.0000000000000000\end{array}$ 
$\begin{array}{llll}0.5000000000000000 & 0.1666410571604189 & 0.0000000000000000\end{array}$

$\begin{array}{llll}0.8999520919833941 & 0.1666410571604189 & 0.0000000000000000\end{array}$

$\begin{array}{llll}0.0000000000000000 & 0.8888291333743084 & 0.0511249999999990\end{array}$

$\begin{array}{llll}0.2000159693388710 & 0.8888291333743084 & 0.0511249999999990\end{array}$

$\begin{array}{llll}0.3999520919833941 & 0.8888291333743084 & 0.0511249999999990\end{array}$

$\begin{array}{llll}0.0000000000000000 & 0.5554701905347272 & 0.0511249999999990\end{array}$

$\begin{array}{llll}0.2000159693388710 & 0.5554701905347272 & 0.0511249999999990\end{array}$

$\begin{array}{llll}0.0999680613222580 & 0.7221880762138895 & 0.0511249999999990\end{array}$

$\begin{array}{llll}0.0000000000000000 & 0.2221880762138895 & 0.0511249999999990\end{array}$

$\begin{array}{llll}0.5999680613222580 & 0.8888291333743084 & 0.0511249999999990\end{array}$

$\begin{array}{llll}0.7999840306611290 & 0.8888291333743084 & 0.0511249999999990\end{array}$

$\begin{array}{llll}0.3999520919833941 & 0.5554701905347272 & 0.0511249999999990\end{array}$

$\begin{array}{llll}0.5999680613222580 & 0.5554701905347272 & 0.0511249999999990\end{array}$

$\begin{array}{llll}0.5000000000000000 & 0.7221880762138895 & 0.0511249999999990\end{array}$

$\begin{array}{llll}0.2000159693388710 & 0.2221880762138895 & 0.0511249999999990\end{array}$

$\begin{array}{llll}0.3999520919833941 & 0.2221880762138895 & 0.0511249999999990\end{array}$

$\begin{array}{llll}0.2999840306611290 & 0.3888291333743084 & 0.0511249999999990\end{array}$

$\begin{array}{llll}0.2999840306611290 & 0.7221880762138895 & 0.0511249999999990\end{array}$

$\begin{array}{llll}0.0999680613222580 & 0.0555470190534706 & 0.0511249999999990\end{array}$

$\begin{array}{llll}0.0999680613222580 & 0.3888291333743084 & 0.0511249999999990\end{array}$

$\begin{array}{llll}0.7999840306611290 & 0.5554701905347272 & 0.0511249999999990\end{array}$

$\begin{array}{llll}0.8999520919833941 & 0.7221880762138895 & 0.0511249999999990\end{array}$

$\begin{array}{llll}0.5999680613222580 & 0.2221880762138895 & 0.0511249999999990\end{array}$

$\begin{array}{llll}0.7999840306611290 & 0.2221880762138895 & 0.0511249999999990\end{array}$

$\begin{array}{llll}0.7000159693388710 & 0.3888291333743084 & 0.0511249999999990\end{array}$

$\begin{array}{llll}0.7000159693388710 & 0.7221880762138895 & 0.0511249999999990\end{array}$

$\begin{array}{llll}0.5000000000000000 & 0.0555470190534706 & 0.0511249999999990\end{array}$

$\begin{array}{llll}0.5000000000000000 & 0.3888291333743084 & 0.0511249999999990\end{array}$

$\begin{array}{llll}0.2999840306611290 & 0.0555470190534706 & 0.0511249999999990\end{array}$

$\begin{array}{llll}0.8999520919833941 & 0.0555470190534706 & 0.0511249999999990\end{array}$

$\begin{array}{lllll}0.8999520919833941 & 0.3888291333743084 & 0.0511249999999990\end{array}$

$\begin{array}{llll}0.7000159693388710 & 0.0555470190534706 & 0.0511249999999990\end{array}$

$\begin{array}{llll}0.5001702454208007 & 0.6121224321929124 & 0.1022796685547969\end{array}$

$\begin{array}{llll}0.3998460283046569 & 0.7778495682505160 & 0.1023654377599435\end{array}$

$\begin{array}{llll}0.6002148221501220 & 0.7773795224280483 & 0.1025221158040264\end{array}$

$\begin{array}{llll}0.6997878523955416 & 0.6111608800855186 & 0.1027819169292445\end{array}$

$\begin{array}{llll}0.3000470117437208 & 0.6116648625609182 & 0.1022919579222500\end{array}$

$\begin{array}{llll}0.1995793044276556 & 0.4435586107466974 & 0.1024896030737524\end{array}$

$\begin{array}{llll}0.8002638368389141 & 0.4445253924031418 & 0.1023150933269190\end{array}$

$\begin{array}{llll}0.3996964990657076 & 0.4445734839008298 & 0.1012586036307449\end{array}$

$\begin{array}{llll}0.6014293282858710 & 0.4450928498510786 & 0.1023228143147178\end{array}$

$\begin{array}{llll}0.9008220581338690 & 0.2772338301287932 & 0.1019546161706067\end{array}$

$\begin{array}{llll}0.1002628951799752 & 0.2776850291760195 & 0.1026079562145158\end{array}$

$\begin{array}{llll}0.5000835503610298 & 0.9446612781553154 & 0.1021159444394593\end{array}$

$\begin{array}{llll}0.8005830269369261 & 0.1105996902016264 & 0.1020838109631982\end{array}$

$\begin{array}{llll}0.2000376940759597 & 0.1108899608718808 & 0.1024792033642366\end{array}$

$\begin{array}{llll}0.0000054128402889 & 0.1107725247424965 & 0.1022057761856101\end{array}$ 
0.70031194155191860 .94437549100424490 .1021858799393622 $\begin{array}{llll}0.2997160960589861 & 0.9443718552377705 & 0.1022182167130712\end{array}$ $\begin{array}{llll}0.0997599449436605 & 0.9441352169196970 & 0.1022397619478407\end{array}$ $\begin{array}{llll}0.9000726834839187 & 0.9441315767110386 & 0.1022803606693265\end{array}$ $\begin{array}{llll}0.5996193943697922 & 0.1110318324785598 & 0.1025076683404312\end{array}$ $\begin{array}{llll}0.3999747420269519 & 0.1113592702617789 & 0.1026612327298380\end{array}$ $\begin{array}{llll}0.2988936856192776 & 0.2772516619812557 & 0.1022709178556694\end{array}$ $\begin{array}{llll}0.7007078076111751 & 0.2772314242314860 & 0.1019849325481913\end{array}$ $\begin{array}{llll}0.8000491792816274 & 0.7778407758436044 & 0.1022478133258730\end{array}$ $\begin{array}{llll}1.0001276125080543 & 0.4441752129965185 & 0.1021154764653832\end{array}$ $\begin{array}{llll}0.1997032802459612 & 0.7778474282850877 & 0.1023082297548484\end{array}$ $\begin{array}{llll}0.9999745490426117 & 0.7775720784851359 & 0.1022699571890017\end{array}$ $\begin{array}{llll}0.5002010579606173 & 0.2764368128925712 & 0.1022825035578901\end{array}$ $\begin{array}{llll}0.9000601266081610 & 0.6110250622404393 & 0.1022518049079940\end{array}$ $\begin{array}{llll}0.0997664891006205 & 0.6109944201383543 & 0.1022297978154218\end{array}$ $\begin{array}{llll}0.5989847219303180 & 0.6674545692991515 & 0.1554730257183066\end{array}$ $\begin{array}{llll}0.3986500620340906 & 0.6684108220924239 & 0.1547741827575050\end{array}$ $\begin{array}{llll}0.2977937977589097 & 0.5016162357076595 & 0.1529030148004247\end{array}$ $\begin{array}{llll}0.7041918308902829 & 0.5014133437930148 & 0.1547397274138325\end{array}$ $\begin{array}{llll}0.4973726170211798 & 0.4997965469049346 & 0.1513878385596124\end{array}$ $\begin{array}{llll}0.4998275792265068 & 0.8343342242134524 & 0.1539009810368851\end{array}$ $\begin{array}{llll}0.0988766222749199 & 0.1661613611976157 & 0.1542941319882446\end{array}$ $\begin{array}{llll}0.9004976110252827 & 0.1663023412773237 & 0.1545812516210861\end{array}$ $0.1995317431535530-0.00018479460824910 .1545536004808326$ $0.8002878432317008-0.00013485976694960 .1545841142042852$ $\begin{array}{lll}-0.0001259348761488 & -0.0002194183397852 & 0.1543888272481253\end{array}$ $\begin{array}{llll}-0.0001516527296199 & 0.3333753886443359 & 0.1544817256867343\end{array}$ $\begin{array}{llll}0.7001809677876122 & 0.8333530449549390 & 0.1546152651730801\end{array}$ $\begin{array}{llll}0.2993618293684505 & 0.8337158606087631 & 0.1546019008054841\end{array}$ $0.6009836471045311-0.00111624654233360 .1541948418385343$ $0.3992109902130725-0.00113452169751780 .1540963144928240$ $\begin{array}{llll}0.2985817557272766 & 0.1660739837879047 & 0.1548096549864613\end{array}$ $\begin{array}{llll}0.7022520517348316 & 0.1656932992309301 & 0.1538329131253200\end{array}$ $\begin{array}{llll}0.1986730325121935 & 0.3334677732516883 & 0.1558950059255888\end{array}$ $\begin{array}{llll}0.8023047858098884 & 0.3319387187292707 & 0.1536159671041373\end{array}$ $\begin{array}{llll}0.9001683924431397 & 0.8336488681369174 & 0.1545403569702581\end{array}$ $\begin{array}{llll}0.0995748890593076 & 0.8335081653266894 & 0.1544667428602542\end{array}$ $\begin{array}{llll}0.5003827383778834 & 0.1630487679047717 & 0.1551037386532894\end{array}$ $\begin{array}{llll}0.3987361595739972 & 0.3344463681738054 & 0.1512138776685122\end{array}$ $\begin{array}{llll}0.6023782219202313 & 0.3323176271560085 & 0.1522502620182583\end{array}$ $\begin{array}{llll}0.0994011686780288 & 0.5003400378888403 & 0.1544872626711091\end{array}$ $\begin{array}{llll}0.8010631950579821 & 0.6680583745312975 & 0.1542905900990937\end{array}$ $\begin{array}{llll}0.9019813961159577 & 0.4999789837433578 & 0.1541975736657176\end{array}$ $\begin{array}{llll}0.1990257185479712 & 0.6672675674358022 & 0.1545301035381702\end{array}$ $\begin{array}{llll}0.9999223496325860 & 0.6669387240669007 & 0.1545812910889052\end{array}$ $\begin{array}{llll}0.3096321547613887 & 0.2874548678944476 & 0.2082718527799489\end{array}$ $\begin{array}{llll}0.5127223945985950 & 0.6066236946391312 & 0.2091526134364851\end{array}$ 
$\begin{array}{llll}0.4952836816784227 & 0.1748768477853014 & 0.2092083963961230\end{array}$

$\begin{array}{llll}0.7000220671486125 & 0.4994196851967408 & 0.2091747412410527\end{array}$

$\begin{array}{llll}0.4067011264541902 & 0.4496498469434669 & 0.2106841347457849\end{array}$

$\begin{array}{llll}0.5005962560418969 & 0.2835218753453640 & 0.2132727928522480\end{array}$

$\begin{array}{llll}0.4001110504697667 & 0.3386721364332991 & 0.2111778490101426\end{array}$

$\begin{array}{llll}0.6029091609577367 & 0.4444083819933444 & 0.2132566702315908\end{array}$

$\begin{array}{llll}0.5994143702001464 & 0.3358940554896013 & 0.2144373296018174\end{array}$

$\begin{array}{llll}0.5060882633563216 & 0.5057191392693443 & 0.2114735703692379\end{array}$

0.33243801308852130 .49341169487324690 .2127772970089744

$\begin{array}{llll}0.6738910355821660 & 0.2922063874480034 & 0.2158169083745357\end{array}$

$\begin{array}{llll}0.6929778121074317 & 0.5742318392818171 & 0.2166183049263816\end{array}$

$\begin{array}{llll}0.7673854439406953 & 0.4646816734230182 & 0.2177651962869683\end{array}$

$\begin{array}{lllll}0.5580111781774164 & 0.1346125797182928 & 0.2186678529418839\end{array}$

$\begin{array}{llll}0.4231716116473140 & 0.1463352855307780 & 0.2167286164691877\end{array}$

Zwitterion on $\mathrm{Cu}$ - VASP - revPBE+D3-calculated lattice - (B - 0 site) $\mathrm{Cu} \mathrm{O} \mathrm{N} \mathrm{C} \mathrm{H}$

1.00000000000000

$\begin{array}{lll}12.5239999999999991 & 0.0000000000000000 & 0.0000000000000000\end{array}$

$\begin{array}{lll}0.0000000000000000 & 13.0160000000000000 & 0.0000000000000000\end{array}$

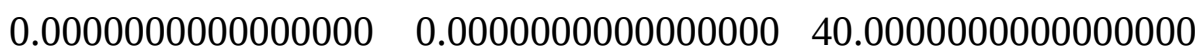

$\mathrm{Cu} \mathrm{O} \quad \mathrm{N} \quad \mathrm{C} \quad \mathrm{H}$

$\begin{array}{lllll}120 & 2 & 2 & 6 & 6\end{array}$

Direct

$\begin{array}{llll}0.0000000000000000 & 0.6666410571604189 & 0.0000000000000000\end{array}$

$\begin{array}{lllll}0.2000159693388710 & 0.6666410571604189 & 0.0000000000000000\end{array}$

$\begin{array}{llllll}0.3999520919833941 & 0.6666410571604189 & 0.0000000000000000\end{array}$

$\begin{array}{lllll}0.2999840306611290 & 0.8332821143208378 & 0.0000000000000000\end{array}$

$\begin{array}{lllll}0.0000000000000000 & 0.3332821143208378 & 0.0000000000000000\end{array}$

$\begin{array}{lllll}0.2000159693388710 & 0.3332821143208378 & 0.0000000000000000\end{array}$

$\begin{array}{lllll}0.0999680613222580 & 0.4999231714812566 & 0.0000000000000000\end{array}$

$\begin{array}{lllll}0.0999680613222580 & 0.8332821143208378 & 0.0000000000000000\end{array}$

$\begin{array}{lllll}0.0000000000000000 & 0.0000000000000000 & 0.0000000000000000\end{array}$

$\begin{array}{lllll}0.5999680613222580 & 0.6666410571604189 & 0.0000000000000000\end{array}$

$\begin{array}{lllll}0.7999840306611290 & 0.6666410571604189 & 0.0000000000000000\end{array}$

$\begin{array}{llll}0.7000159693388710 & 0.8332821143208378 & 0.0000000000000000\end{array}$

$\begin{array}{llllll}0.3999520919833941 & 0.3332821143208378 & 0.0000000000000000\end{array}$

$\begin{array}{lllll}0.5999680613222580 & 0.3332821143208378 & 0.0000000000000000\end{array}$

$\begin{array}{lllll}0.5000000000000000 & 0.4999231714812566 & 0.0000000000000000\end{array}$

$\begin{array}{lllll}0.5000000000000000 & 0.8332821143208378 & 0.0000000000000000\end{array}$

$\begin{array}{llll}0.2000159693388710 & 0.0000000000000000 & 0.0000000000000000\end{array}$

$\begin{array}{llllll}0.3999520919833941 & 0.0000000000000000 & 0.0000000000000000\end{array}$

$\begin{array}{lllll}0.2999840306611290 & 0.1666410571604189 & 0.0000000000000000\end{array}$

$\begin{array}{lllll}0.2999840306611290 & 0.4999231714812566 & 0.0000000000000000\end{array}$

$\begin{array}{llllll}0.0999680613222580 & 0.1666410571604189 & 0.0000000000000000\end{array}$

$\begin{array}{lllll}0.7999840306611290 & 0.3332821143208378 & 0.0000000000000000\end{array}$

$\begin{array}{lllll}0.8999520919833941 & 0.4999231714812566 & 0.0000000000000000\end{array}$ 
$\begin{array}{lll}0.8999520919833941 & 0.8332821143208378 & 0.0000000000000000\end{array}$

$\begin{array}{llll}0.5999680613222580 & 0.0000000000000000 & 0.0000000000000000\end{array}$

$\begin{array}{llll}0.7999840306611290 & 0.0000000000000000 & 0.0000000000000000\end{array}$

$\begin{array}{llll}0.7000159693388710 & 0.1666410571604189 & 0.0000000000000000\end{array}$

$\begin{array}{lll}0.7000159693388710 & 0.4999231714812566 & 0.0000000000000000\end{array}$

$\begin{array}{llll}0.5000000000000000 & 0.1666410571604189 & 0.0000000000000000\end{array}$

$\begin{array}{llll}0.8999520919833941 & 0.1666410571604189 & 0.0000000000000000\end{array}$

$\begin{array}{llll}0.0000000000000000 & 0.8888291333743084 & 0.0511249999999990\end{array}$

$\begin{array}{llll}0.2000159693388710 & 0.8888291333743084 & 0.0511249999999990\end{array}$

$\begin{array}{llll}0.3999520919833941 & 0.8888291333743084 & 0.0511249999999990\end{array}$

$\begin{array}{llll}0.0000000000000000 & 0.5554701905347272 & 0.0511249999999990\end{array}$

$\begin{array}{llll}0.2000159693388710 & 0.5554701905347272 & 0.0511249999999990\end{array}$

$\begin{array}{llll}0.0999680613222580 & 0.7221880762138895 & 0.0511249999999990\end{array}$

$\begin{array}{llll}0.0000000000000000 & 0.2221880762138895 & 0.0511249999999990\end{array}$

$\begin{array}{llll}0.5999680613222580 & 0.8888291333743084 & 0.0511249999999990\end{array}$

$\begin{array}{llll}0.7999840306611290 & 0.8888291333743084 & 0.0511249999999990\end{array}$

$\begin{array}{llll}0.3999520919833941 & 0.5554701905347272 & 0.0511249999999990\end{array}$

$\begin{array}{llll}0.5999680613222580 & 0.5554701905347272 & 0.0511249999999990\end{array}$

$\begin{array}{llll}0.5000000000000000 & 0.7221880762138895 & 0.0511249999999990\end{array}$

$\begin{array}{llll}0.2000159693388710 & 0.2221880762138895 & 0.0511249999999990\end{array}$

$\begin{array}{llll}0.3999520919833941 & 0.2221880762138895 & 0.0511249999999990\end{array}$

$\begin{array}{llll}0.2999840306611290 & 0.3888291333743084 & 0.0511249999999990\end{array}$

$0.2999840306611290 \quad 0.72218807621388950 .0511249999999990$

$\begin{array}{llll}0.0999680613222580 & 0.0555470190534706 & 0.0511249999999990\end{array}$

$\begin{array}{llll}0.0999680613222580 & 0.3888291333743084 & 0.0511249999999990\end{array}$

$\begin{array}{llll}0.7999840306611290 & 0.5554701905347272 & 0.0511249999999990\end{array}$

$\begin{array}{llll}0.8999520919833941 & 0.7221880762138895 & 0.0511249999999990\end{array}$

$\begin{array}{llll}0.5999680613222580 & 0.2221880762138895 & 0.0511249999999990\end{array}$

$\begin{array}{llll}0.7999840306611290 & 0.2221880762138895 & 0.0511249999999990\end{array}$

$\begin{array}{llll}0.7000159693388710 & 0.3888291333743084 & 0.0511249999999990\end{array}$

$\begin{array}{llll}0.7000159693388710 & 0.7221880762138895 & 0.0511249999999990\end{array}$

$\begin{array}{llll}0.5000000000000000 & 0.0555470190534706 & 0.0511249999999990\end{array}$

$\begin{array}{llll}0.5000000000000000 & 0.3888291333743084 & 0.0511249999999990\end{array}$

$\begin{array}{llll}0.2999840306611290 & 0.0555470190534706 & 0.0511249999999990\end{array}$

$\begin{array}{llll}0.8999520919833941 & 0.0555470190534706 & 0.0511249999999990\end{array}$

$\begin{array}{lllll}0.8999520919833941 & 0.3888291333743084 & 0.0511249999999990\end{array}$

$\begin{array}{llll}0.7000159693388710 & 0.0555470190534706 & 0.0511249999999990\end{array}$

$\begin{array}{llll}0.4999922245425804 & 0.6115483568260480 & 0.1022764435153556\end{array}$

$\begin{array}{llll}0.3998278441113269 & 0.7780826291897602 & 0.1022179546077725\end{array}$

$\begin{array}{llll}0.6000382554757158 & 0.7780637579049645 & 0.1022146909877905\end{array}$

$\begin{array}{llll}0.7002976981557387 & 0.6103452786387069 & 0.1031489835302838\end{array}$

$\begin{array}{llll}0.2996959785335253 & 0.6102885420583082 & 0.1031843784735672\end{array}$

$\begin{array}{llll}0.2004031546221677 & 0.4458248222805476 & 0.1024994424600768\end{array}$

$\begin{array}{llll}0.7996246896440812 & 0.4456313662698216 & 0.1025508958916336\end{array}$

$\begin{array}{llll}0.3988214256825859 & 0.4462751111104281 & 0.1017795255761857\end{array}$

0.60132524780856340 .44614510154480540 .1017555461237846

$\begin{array}{llll}0.9003402141701902 & 0.2775407251494083 & 0.1019527842741356\end{array}$ 
$\begin{array}{llll}0.0993829608719236 & 0.2775222487951227 & 0.1018395630967623\end{array}$ $\begin{array}{llll}0.4999221028980623 & 0.9445223186952458 & 0.1021433412539864\end{array}$ $\begin{array}{llll}0.7996797230741727 & 0.1112186603865760 & 0.1027741458257597\end{array}$ $\begin{array}{llll}0.2002093067602476 & 0.1112559474553549 & 0.1027801075180303\end{array}$ $\begin{array}{llll}-0.0000723672977466 & 0.1105922416919336 & 0.1021038233943643\end{array}$ $\begin{array}{llll}0.7001795032136295 & 0.9443333696755005 & 0.1022829443875782\end{array}$ $\begin{array}{llll}0.2997254616389429 & 0.9443843573546729 & 0.1022953102561475\end{array}$ $\begin{array}{llll}0.0999585086773676 & 0.9443528528856184 & 0.1022520901989639\end{array}$ $\begin{array}{llll}0.8999869201347848 & 0.9443363075328586 & 0.1022515034313369\end{array}$ $\begin{array}{llll}0.6014957207658546 & 0.1110784856774953 & 0.1025544926235707\end{array}$ $\begin{array}{llll}0.3984166116060196 & 0.1110826311311549 & 0.1025570108258544\end{array}$ $\begin{array}{llll}0.2988019717691395 & 0.2770355186205536 & 0.1018868578033721\end{array}$ $\begin{array}{llll}0.7011855006366807 & 0.2769645350905831 & 0.1020153313675925\end{array}$ $\begin{array}{llll}0.7998929704256721 & 0.7777861962408219 & 0.1022702092594553\end{array}$ $\begin{array}{llll}0.9999501560607235 & 0.4445646365256098 & 0.1021179710844460\end{array}$ $\begin{array}{llll}0.2000073868067578 & 0.7778319552393911 & 0.1022595440073330\end{array}$ $\begin{array}{llll}0.9999258117357323 & 0.7779263045891108 & 0.1022635816206774\end{array}$ $\begin{array}{llll}0.5000607308422956 & 0.2772785097086696 & 0.1008747132743848\end{array}$ $\begin{array}{llll}0.9000021459653084 & 0.6114404425598780 & 0.1022745263785919\end{array}$ $\begin{array}{llll}0.0999538960797471 & 0.6114505649815619 & 0.1022732143485990\end{array}$ $\begin{array}{llll}0.6001333410799692 & 0.6674099504803163 & 0.1542250922289585\end{array}$ $\begin{array}{llll}0.4001794815088907 & 0.6673501887892483 & 0.1541775993902178\end{array}$ 0.29752993041550230 .50058930650642060 .1579535889997772 $\begin{array}{llll}0.7031674810149920 & 0.5007159227578937 & 0.1578444275388337\end{array}$ 0.50073894611536120 .50095516470753040 .1525912903064872 $\begin{array}{llll}0.4999836831122612 & 0.8337769701344957 & 0.1544038173035238\end{array}$ $\begin{array}{llll}0.0984000498456676 & 0.1667516609731085 & 0.1543307682181722\end{array}$ $\begin{array}{llll}0.9013388038272311 & 0.1667729032142880 & 0.1543433364027725\end{array}$ $0.1998330260918357-0.00005479925434700 .1544875788850802$ $0.8001696265622386-0.00017143435569140 .1544577493128928$ $\begin{array}{llll}-0.0000139722045268 & 0.0000941446139300 & 0.1544173829368918\end{array}$ $\begin{array}{llll}-0.0000320883638771 & 0.3334813888755211 & 0.1544842818195324\end{array}$ $\begin{array}{llll}0.6999836149226835 & 0.8337386456319456 & 0.1545065166998758\end{array}$ 0.30001158090647690 .83373307825595330 .1545042848902376 $\begin{array}{llll}0.5999975205720409 & 0.0000312742709655 & 0.1543039688761187\end{array}$ $\begin{array}{llll}0.3998386372779489 & 0.0001757781820401 & 0.1543724470220994\end{array}$ 0.29690671993354250 .16817095014757190 .1565881710362843 $\begin{array}{llll}0.7026346073923346 & 0.1677317750116453 & 0.1565081482580461\end{array}$ $\begin{array}{llll}0.1964350676839382 & 0.3345539368792411 & 0.1522847157968626\end{array}$ $\begin{array}{llll}0.8032401406883771 & 0.3346203619876919 & 0.1526665724944657\end{array}$ $\begin{array}{llll}0.9001448989794636 & 0.8338412253929233 & 0.1544959953153106\end{array}$ $\begin{array}{llll}0.0998323488585021 & 0.8338649046413091 & 0.1544889344171586\end{array}$ 0.49968725875308290 .16592151982454540 .1522448276393498 $\begin{array}{llll}0.4003082767314159 & 0.3335949376094962 & 0.1499128307102348\end{array}$ $\begin{array}{llll}0.5997257317399718 & 0.3337379741010972 & 0.1500848277566727\end{array}$ $\begin{array}{llll}0.0982665217415647 & 0.5012198119341321 & 0.1541496044767520\end{array}$ $\begin{array}{llll}0.8007423092790200 & 0.6678077757894616 & 0.1545049719766629\end{array}$ 
$\begin{array}{llll}0.9019494387051430 & 0.5012721605050570 & 0.1541927851766693\end{array}$

$\begin{array}{llll}0.1992937296800997 & 0.6677689652945982 & 0.1544450428785679\end{array}$

$\begin{array}{llll}1.0000232444414259 & 0.6678382930258433 & 0.1544637703086854\end{array}$

$\begin{array}{llll}0.3087346786578042 & 0.4380924673573812 & 0.2077619129418716\end{array}$

$\begin{array}{lll}0.6981644197758512 & 0.4303642473432402 & 0.2092069401742122\end{array}$

$\begin{array}{llll}0.3026658177509022 & 0.2228945188447724 & 0.2103223326713084\end{array}$

$\begin{array}{llll}0.6950044224857850 & 0.2154461158443040 & 0.2101283153201409\end{array}$

$\begin{array}{llll}0.5035612711135573 & 0.4386410140148174 & 0.2100348178116418\end{array}$

$\begin{array}{llll}0.4016149877026436 & 0.2771516464020510 & 0.2134297832710157\end{array}$

$\begin{array}{llll}0.4005963552415404 & 0.3881563797947176 & 0.2106622978998386\end{array}$

$\begin{array}{llll}0.5981274033904681 & 0.2730857443097900 & 0.2133938960085834\end{array}$

$\begin{array}{llll}0.4988394024990050 & 0.2223909563610384 & 0.2151552007784495\end{array}$

$\begin{array}{llll}0.6042922246491063 & 0.3840836955882582 & 0.2111604546974381\end{array}$

$\begin{array}{lll}0.5054410098480033 & 0.5223187384948353 & 0.2130344885449073\end{array}$

$\begin{array}{llll}0.4969643799262258 & 0.1384878728056094 & 0.2170153380167590\end{array}$

$\begin{array}{llll}0.7596260316645358 & 0.2606084923805324 & 0.2154824142409074\end{array}$

$\begin{array}{llll}0.6968174788397525 & 0.1485413414812704 & 0.2232791703165628\end{array}$

$\begin{array}{llll}0.2979933506570986 & 0.1568955581044436 & 0.2238722029918190\end{array}$

$\begin{array}{llll}0.2395902567923450 & 0.2704927220667618 & 0.2152472994853129\end{array}$

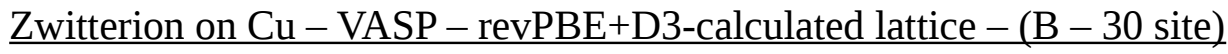

$\mathrm{Cu} \mathrm{O} \mathrm{N} \mathrm{C} \mathrm{H}$

1.00000000000000

$\begin{array}{lll}12.5239999999999991 & 0.0000000000000000 & 0.0000000000000000\end{array}$

$\begin{array}{llll}0.0000000000000000 & 13.0160000000000000 & 0.0000000000000000\end{array}$

$0.0000000000000000 \quad 0.0000000000000000 \quad 40.0000000000000000$

$\mathrm{Cu} \quad \mathrm{O} \quad \mathrm{N} \quad \mathrm{C} \quad \mathrm{H}$

$\begin{array}{lllll}120 & 2 & 2 & 6 & 6\end{array}$

Direct

$\begin{array}{llll}0.0000000000000000 & 0.6666410571604189 & 0.0000000000000000\end{array}$

$\begin{array}{llll}0.2000159693388710 & 0.6666410571604189 & 0.0000000000000000\end{array}$

$\begin{array}{llll}0.3999520919833941 & 0.6666410571604189 & 0.0000000000000000\end{array}$

$\begin{array}{lll}0.2999840306611290 & 0.8332821143208378 & 0.0000000000000000\end{array}$

$\begin{array}{llll}0.0000000000000000 & 0.3332821143208378 & 0.0000000000000000\end{array}$

$\begin{array}{llll}0.2000159693388710 & 0.3332821143208378 & 0.0000000000000000\end{array}$

$\begin{array}{llll}0.0999680613222580 & 0.4999231714812566 & 0.0000000000000000\end{array}$

$\begin{array}{llll}0.0999680613222580 & 0.8332821143208378 & 0.0000000000000000\end{array}$

$\begin{array}{lll}0.0000000000000000 & 0.0000000000000000 & 0.0000000000000000\end{array}$

$\begin{array}{lll}0.5999680613222580 & 0.6666410571604189 & 0.0000000000000000\end{array}$

$\begin{array}{llll}0.7999840306611290 & 0.6666410571604189 & 0.0000000000000000\end{array}$

$\begin{array}{lllll}0.7000159693388710 & 0.8332821143208378 & 0.0000000000000000\end{array}$

$\begin{array}{llll}0.3999520919833941 & 0.3332821143208378 & 0.0000000000000000\end{array}$

$\begin{array}{llll}0.5999680613222580 & 0.3332821143208378 & 0.0000000000000000\end{array}$

$\begin{array}{llll}0.5000000000000000 & 0.4999231714812566 & 0.0000000000000000\end{array}$

$\begin{array}{llll}0.5000000000000000 & 0.8332821143208378 & 0.0000000000000000\end{array}$

$\begin{array}{lllll}0.2000159693388710 & 0.0000000000000000 & 0.0000000000000000\end{array}$

$\begin{array}{llll}0.3999520919833941 & 0.0000000000000000 & 0.0000000000000000\end{array}$ 
$\begin{array}{lll}0.2999840306611290 & 0.1666410571604189 & 0.0000000000000000\end{array}$

$\begin{array}{llll}0.2999840306611290 & 0.4999231714812566 & 0.0000000000000000\end{array}$

$\begin{array}{llll}0.0999680613222580 & 0.1666410571604189 & 0.0000000000000000\end{array}$

$\begin{array}{llll}0.7999840306611290 & 0.3332821143208378 & 0.0000000000000000\end{array}$

$\begin{array}{llll}0.8999520919833941 & 0.4999231714812566 & 0.0000000000000000\end{array}$

$\begin{array}{lllll}0.8999520919833941 & 0.8332821143208378 & 0.0000000000000000\end{array}$

$\begin{array}{llll}0.5999680613222580 & 0.0000000000000000 & 0.0000000000000000\end{array}$

$\begin{array}{llll}0.7999840306611290 & 0.0000000000000000 & 0.0000000000000000\end{array}$

$\begin{array}{llll}0.7000159693388710 & 0.1666410571604189 & 0.0000000000000000\end{array}$

$\begin{array}{lll}0.7000159693388710 & 0.4999231714812566 & 0.0000000000000000\end{array}$

$\begin{array}{lllll}0.5000000000000000 & 0.1666410571604189 & 0.0000000000000000\end{array}$

$\begin{array}{llll}0.8999520919833941 & 0.1666410571604189 & 0.0000000000000000\end{array}$

$\begin{array}{llll}0.0000000000000000 & 0.8888291333743084 & 0.0511249999999990\end{array}$

$\begin{array}{llll}0.2000159693388710 & 0.8888291333743084 & 0.0511249999999990\end{array}$

$\begin{array}{llll}0.3999520919833941 & 0.8888291333743084 & 0.0511249999999990\end{array}$

$\begin{array}{llll}0.0000000000000000 & 0.5554701905347272 & 0.0511249999999990\end{array}$

$\begin{array}{llll}0.2000159693388710 & 0.5554701905347272 & 0.0511249999999990\end{array}$

$\begin{array}{llll}0.0999680613222580 & 0.7221880762138895 & 0.0511249999999990\end{array}$

$\begin{array}{llll}0.0000000000000000 & 0.2221880762138895 & 0.0511249999999990\end{array}$

$\begin{array}{llll}0.5999680613222580 & 0.8888291333743084 & 0.0511249999999990\end{array}$

$\begin{array}{llll}0.7999840306611290 & 0.8888291333743084 & 0.0511249999999990\end{array}$

$\begin{array}{llll}0.3999520919833941 & 0.5554701905347272 & 0.0511249999999990\end{array}$

$\begin{array}{llll}0.5999680613222580 & 0.5554701905347272 & 0.0511249999999990\end{array}$

$\begin{array}{llll}0.5000000000000000 & 0.7221880762138895 & 0.0511249999999990\end{array}$

$\begin{array}{llll}0.2000159693388710 & 0.2221880762138895 & 0.0511249999999990\end{array}$

$\begin{array}{lllll}0.3999520919833941 & 0.2221880762138895 & 0.0511249999999990\end{array}$

$\begin{array}{llll}0.2999840306611290 & 0.3888291333743084 & 0.0511249999999990\end{array}$

$\begin{array}{lll}0.2999840306611290 & 0.7221880762138895 & 0.0511249999999990\end{array}$

$\begin{array}{llll}0.0999680613222580 & 0.0555470190534706 & 0.0511249999999990\end{array}$

$\begin{array}{llll}0.0999680613222580 & 0.3888291333743084 & 0.0511249999999990\end{array}$

$\begin{array}{llll}0.7999840306611290 & 0.5554701905347272 & 0.0511249999999990\end{array}$

$\begin{array}{llll}0.8999520919833941 & 0.7221880762138895 & 0.0511249999999990\end{array}$

$\begin{array}{llll}0.5999680613222580 & 0.2221880762138895 & 0.0511249999999990\end{array}$

$\begin{array}{llll}0.7999840306611290 & 0.2221880762138895 & 0.0511249999999990\end{array}$

$\begin{array}{llll}0.7000159693388710 & 0.3888291333743084 & 0.0511249999999990\end{array}$

$\begin{array}{llll}0.7000159693388710 & 0.7221880762138895 & 0.0511249999999990\end{array}$

$\begin{array}{llll}0.5000000000000000 & 0.0555470190534706 & 0.0511249999999990\end{array}$

$\begin{array}{llll}0.5000000000000000 & 0.3888291333743084 & 0.0511249999999990\end{array}$

$\begin{array}{llll}0.2999840306611290 & 0.0555470190534706 & 0.0511249999999990\end{array}$

$\begin{array}{llll}0.8999520919833941 & 0.0555470190534706 & 0.0511249999999990\end{array}$

$\begin{array}{llll}0.8999520919833941 & 0.3888291333743084 & 0.0511249999999990\end{array}$

$\begin{array}{llll}0.7000159693388710 & 0.0555470190534706 & 0.0511249999999990\end{array}$

$\begin{array}{llll}0.5001077710712918 & 0.6115096124305027 & 0.1022564603522934\end{array}$

$\begin{array}{llll}0.3998187749307482 & 0.7780175103592836 & 0.1021586311988488\end{array}$

$\begin{array}{llll}0.6000867639287657 & 0.7777786047598871 & 0.1022566721704627\end{array}$

$\begin{array}{lllll}0.7000810761918990 & 0.6106729716095716 & 0.1028047760697857\end{array}$

$\begin{array}{llll}0.2992630272433858 & 0.6117448087893645 & 0.1020029492029755\end{array}$ 
0.19934885911898940 .44295133818732020 .1028237359846530 $\begin{array}{llll}0.7991634494352796 & 0.4441347209755014 & 0.1035230437269175\end{array}$ $\begin{array}{llll}0.3990380261764705 & 0.4452999034537318 & 0.1013335624330576\end{array}$ $\begin{array}{llll}0.6010139386107778 & 0.4456106740726358 & 0.1022057859998771\end{array}$ $\begin{array}{llll}0.8994343406606932 & 0.2783871735386394 & 0.1028207248816550\end{array}$ $\begin{array}{llll}0.1007344092279322 & 0.2782182152070214 & 0.1031647430191820\end{array}$ $\begin{array}{llll}0.5002269803360773 & 0.9439318446808376 & 0.1020347675502438\end{array}$ 0.80124504573395320 .11064231280290630 .1018586557001773 $\begin{array}{llll}0.1995909032115021 & 0.1110381881832125 & 0.1024203159455762\end{array}$ $0.0003080877257954 \quad 0.1112433647365454 \quad 0.1021670138282756$ $\begin{array}{llll}0.7005065319709537 & 0.9439746299843136 & 0.1021622645934581\end{array}$ $\begin{array}{llll}0.2993599395745498 & 0.9440631444148226 & 0.1021462653906923\end{array}$ $0.09951114499993860 .9442565070527902 \quad 0.1022083466215868$ $\begin{array}{llll}0.9001877572494466 & 0.9440925747226907 & 0.1022831490458738\end{array}$ $\begin{array}{llll}0.6003992835893519 & 0.1101173847533154 & 0.1014541802273765\end{array}$ $\begin{array}{llll}0.3992344306827458 & 0.1105252453447885 & 0.1018124220327053\end{array}$ $\begin{array}{llll}0.2983152635464688 & 0.2772371031535384 & 0.1026896783691790\end{array}$ $\begin{array}{llll}0.7016119745817605 & 0.2778709657984066 & 0.1019193502859161\end{array}$ $\begin{array}{llll}0.8000464363787969 & 0.7774930577593656 & 0.1022779249100634\end{array}$ $\begin{array}{llll}0.9995723090795511 & 0.4439400727296843 & 0.1023465305246793\end{array}$ $\begin{array}{llll}0.1995753598648831 & 0.7777630710151149 & 0.1022559257192294\end{array}$ $\begin{array}{llll}0.9999176272033875 & 0.7774472007065847 & 0.1022524470054860\end{array}$ $\begin{array}{llll}0.4993828615611791 & 0.2775410034919965 & 0.1010321362942230\end{array}$ $\begin{array}{llll}0.8998230211836874 & 0.6107139345286462 & 0.1022714093341802\end{array}$ $\begin{array}{llll}0.0995707767736601 & 0.6106744840263543 & 0.1022290960130087\end{array}$ $\begin{array}{llll}0.5985197376944752 & 0.6685280479711925 & 0.1543715221540546\end{array}$ $\begin{array}{llll}0.3989171803045535 & 0.6676980338665411 & 0.1540592589881623\end{array}$ $\begin{array}{llll}0.2972945747121321 & 0.5029815603681442 & 0.1530399616698048\end{array}$ $\begin{array}{llll}0.6992165178592975 & 0.5036323354387986 & 0.1564695063154220\end{array}$ $\begin{array}{llll}0.4964891977301215 & 0.5004847805204943 & 0.1525951461747096\end{array}$ $\begin{array}{llll}0.4997462386746180 & 0.8335164454093732 & 0.1544771073613392\end{array}$ $\begin{array}{llll}0.0999087084966034 & 0.1663383632907314 & 0.1545967574237768\end{array}$ $\begin{array}{llll}0.9005050404346318 & 0.1661830928023426 & 0.1543617053928097\end{array}$ $0.1993751980879250-0.00087100158733040 .1544233633061707$ $0.8005165965767540-0.00060428812450260 .1544963738074090$ $\begin{array}{llll}0.0000116046674495 & 0.0000180548232576 & 0.1544501311234927\end{array}$ 0.00002220060336680 .33330246628167650 .1547771427742887 $0.7000402994450405 \quad 0.83348516862202490 .1543765196217983$ $\begin{array}{llll}0.2995920097781952 & 0.8333837410530180 & 0.1544966609630127\end{array}$ $0.5999062211597398-0.00084148577875320 .1542391116035194$ $0.4004675247420382-0.00211293953293880 .1534031928253518$ $\begin{array}{lll}0.3009074998831950 & 0.1627794703332385 & 0.1541386910472420\end{array}$ $\begin{array}{llll}0.7015348307054354 & 0.1626732774095777 & 0.1522069412067308\end{array}$ $\begin{array}{llll}0.2018955475956006 & 0.3325678910493135 & 0.1590474259690240\end{array}$ $\begin{array}{llll}0.7986529309730814 & 0.3324015265186256 & 0.1568631370246137\end{array}$ $0.90032416822551650 .8335579769797806 \quad 0.1544261363668588$ $\begin{array}{llll}0.0994760385756976 & 0.8334194518023897 & 0.1544386359345446\end{array}$ 
0.50211514392268440 .16531079382974520 .1516063329642153

$\begin{array}{llll}0.4002446685636672 & 0.3345247901400445 & 0.1509538144609603\end{array}$

$\begin{array}{llll}0.5992240190165502 & 0.3313724626542481 & 0.1506013951695395\end{array}$

$\begin{array}{llll}0.0989117453546788 & 0.4999054408440300 & 0.1544371802957369\end{array}$

0.80090062301381340 .66777916950559620 .1543674900407181

$\begin{array}{llll}0.9000981478906954 & 0.5000601350830441 & 0.1545247245792502\end{array}$

$\begin{array}{llll}0.1989658059721572 & 0.6674921997251783 & 0.1543656053965679\end{array}$

$\begin{array}{llll}0.9999770707953968 & 0.6667383000649153 & 0.1544415150517860\end{array}$

0.28568976348210750 .33419204355969340 .2098145111953614

$\begin{array}{llll}0.6288588607842601 & 0.5123679937004867 & 0.2092714374107027\end{array}$

0.38380812683093130 .15130679551276540 .2128731493955267

$\begin{array}{llll}0.7360310715454383 & 0.3287849173284388 & 0.2102403267084152\end{array}$

$\begin{array}{llll}0.4542733883695653 & 0.4287816561741586 & 0.2106386645069910\end{array}$

$\begin{array}{llll}0.4480585782831857 & 0.2394356076897266 & 0.2142474943531758\end{array}$

$\begin{array}{llll}0.3909446117161883 & 0.3363398468459596 & 0.2116566905756528\end{array}$

$\begin{array}{llll}0.6215834886366360 & 0.3288234114385317 & 0.2122590937582151\end{array}$

$\begin{array}{llll}0.5610762934277507 & 0.2368938998663052 & 0.2145225337823408\end{array}$

$\begin{array}{llll}0.5699242222781272 & 0.4284867951418606 & 0.2109511043569585\end{array}$

$\begin{array}{lll}0.4128024256644502 & 0.5022482866044083 & 0.2130187517364539\end{array}$

$\begin{array}{llll}0.6024484595291384 & 0.1630897954503566 & 0.2159837338819805\end{array}$

$\begin{array}{llll}0.7659956208974400 & 0.3967039939465859 & 0.2190487009992780\end{array}$

$\begin{array}{llll}0.7720112948176177 & 0.2664604713538526 & 0.2206670564365198\end{array}$

$\begin{array}{llll}0.4174091960456022 & 0.0820895274027016 & 0.2177353207972569\end{array}$

$\begin{array}{lll}0.3076934737347453 & 0.1615175210728843 & 0.2211572662951772\end{array}$

Zwitterion on $\mathrm{Cu}$ - VASP - revPBE+D3-calculated lattice - (B - 60 site) $\mathrm{Cu} \mathrm{O} \mathrm{N} \mathrm{C} \mathrm{H}$

1.00000000000000

$\begin{array}{lll}12.5239999999999991 & 0.0000000000000000 & 0.0000000000000000\end{array}$

$\begin{array}{lll}0.0000000000000000 & 13.0160000000000000 & 0.0000000000000000\end{array}$

$\begin{array}{llll}0.0000000000000000 & 0.0000000000000000 & 40.0000000000000000\end{array}$

$\begin{array}{llllll}\mathrm{Cu} & \mathrm{O} & \mathrm{N} & \mathrm{C} & \mathrm{H}\end{array}$

$\begin{array}{lllll}120 & 2 & 2 & 6 & 6\end{array}$

Direct

$0.0000000000000000 \quad 0.66664105716041890 .0000000000000000$

$\begin{array}{llll}0.2000159693388710 & 0.6666410571604189 & 0.0000000000000000\end{array}$

$\begin{array}{llll}0.3999520919833941 & 0.6666410571604189 & 0.0000000000000000\end{array}$

$\begin{array}{lll}0.2999840306611290 & 0.8332821143208378 & 0.0000000000000000\end{array}$

$\begin{array}{llll}0.0000000000000000 & 0.3332821143208378 & 0.0000000000000000\end{array}$

$\begin{array}{llll}0.2000159693388710 & 0.3332821143208378 & 0.0000000000000000\end{array}$

$\begin{array}{llll}0.0999680613222580 & 0.4999231714812566 & 0.0000000000000000\end{array}$

$\begin{array}{llll}0.0999680613222580 & 0.8332821143208378 & 0.0000000000000000\end{array}$

$\begin{array}{lll}0.0000000000000000 & 0.0000000000000000 & 0.0000000000000000\end{array}$

$\begin{array}{lll}0.5999680613222580 & 0.6666410571604189 & 0.0000000000000000\end{array}$

$\begin{array}{lll}0.7999840306611290 & 0.6666410571604189 & 0.0000000000000000\end{array}$

$\begin{array}{llll}0.7000159693388710 & 0.8332821143208378 & 0.0000000000000000\end{array}$

$\begin{array}{llll}0.3999520919833941 & 0.3332821143208378 & 0.0000000000000000\end{array}$ 
$\begin{array}{lll}0.5999680613222580 & 0.3332821143208378 & 0.0000000000000000\end{array}$

$\begin{array}{llll}0.5000000000000000 & 0.4999231714812566 & 0.0000000000000000\end{array}$

$\begin{array}{llll}0.5000000000000000 & 0.8332821143208378 & 0.0000000000000000\end{array}$

$\begin{array}{lll}0.2000159693388710 & 0.0000000000000000 & 0.0000000000000000\end{array}$

$\begin{array}{llll}0.3999520919833941 & 0.0000000000000000 & 0.0000000000000000\end{array}$

$\begin{array}{llll}0.2999840306611290 & 0.1666410571604189 & 0.0000000000000000\end{array}$

$\begin{array}{llll}0.2999840306611290 & 0.4999231714812566 & 0.0000000000000000\end{array}$

$\begin{array}{llll}0.0999680613222580 & 0.1666410571604189 & 0.0000000000000000\end{array}$

$\begin{array}{llll}0.7999840306611290 & 0.3332821143208378 & 0.0000000000000000\end{array}$

$\begin{array}{llll}0.8999520919833941 & 0.4999231714812566 & 0.0000000000000000\end{array}$

$\begin{array}{llll}0.8999520919833941 & 0.8332821143208378 & 0.0000000000000000\end{array}$

$\begin{array}{llll}0.5999680613222580 & 0.0000000000000000 & 0.0000000000000000\end{array}$

$\begin{array}{llll}0.7999840306611290 & 0.0000000000000000 & 0.0000000000000000\end{array}$

$\begin{array}{llll}0.7000159693388710 & 0.1666410571604189 & 0.0000000000000000\end{array}$

$\begin{array}{llll}0.7000159693388710 & 0.4999231714812566 & 0.0000000000000000\end{array}$

$\begin{array}{llll}0.5000000000000000 & 0.1666410571604189 & 0.0000000000000000\end{array}$

$\begin{array}{llll}0.8999520919833941 & 0.1666410571604189 & 0.0000000000000000\end{array}$

$\begin{array}{llll}0.0000000000000000 & 0.8888291333743084 & 0.0511249999999990\end{array}$

$\begin{array}{llll}0.2000159693388710 & 0.8888291333743084 & 0.0511249999999990\end{array}$

$\begin{array}{llll}0.3999520919833941 & 0.8888291333743084 & 0.0511249999999990\end{array}$

$\begin{array}{llll}0.0000000000000000 & 0.5554701905347272 & 0.0511249999999990\end{array}$

$\begin{array}{llll}0.2000159693388710 & 0.5554701905347272 & 0.0511249999999990\end{array}$

$\begin{array}{llll}0.0999680613222580 & 0.7221880762138895 & 0.0511249999999990\end{array}$

$\begin{array}{llll}0.0000000000000000 & 0.2221880762138895 & 0.0511249999999990\end{array}$

$\begin{array}{llll}0.5999680613222580 & 0.8888291333743084 & 0.0511249999999990\end{array}$

$\begin{array}{llll}0.7999840306611290 & 0.8888291333743084 & 0.0511249999999990\end{array}$

$\begin{array}{llll}0.3999520919833941 & 0.5554701905347272 & 0.0511249999999990\end{array}$

$\begin{array}{llll}0.5999680613222580 & 0.5554701905347272 & 0.0511249999999990\end{array}$

$\begin{array}{llll}0.5000000000000000 & 0.7221880762138895 & 0.0511249999999990\end{array}$

$\begin{array}{llll}0.2000159693388710 & 0.2221880762138895 & 0.0511249999999990\end{array}$

$\begin{array}{llll}0.3999520919833941 & 0.2221880762138895 & 0.0511249999999990\end{array}$

$\begin{array}{llll}0.2999840306611290 & 0.3888291333743084 & 0.0511249999999990\end{array}$

$\begin{array}{llll}0.2999840306611290 & 0.7221880762138895 & 0.0511249999999990\end{array}$

$\begin{array}{llll}0.0999680613222580 & 0.0555470190534706 & 0.0511249999999990\end{array}$

$\begin{array}{llll}0.0999680613222580 & 0.3888291333743084 & 0.0511249999999990\end{array}$

$\begin{array}{llll}0.7999840306611290 & 0.5554701905347272 & 0.0511249999999990\end{array}$

$\begin{array}{llll}0.8999520919833941 & 0.7221880762138895 & 0.0511249999999990\end{array}$

$\begin{array}{llll}0.5999680613222580 & 0.2221880762138895 & 0.0511249999999990\end{array}$

$\begin{array}{llll}0.7999840306611290 & 0.2221880762138895 & 0.0511249999999990\end{array}$

$\begin{array}{llll}0.7000159693388710 & 0.3888291333743084 & 0.0511249999999990\end{array}$

$\begin{array}{llll}0.7000159693388710 & 0.7221880762138895 & 0.0511249999999990\end{array}$

$\begin{array}{llll}0.5000000000000000 & 0.0555470190534706 & 0.0511249999999990\end{array}$

$\begin{array}{llll}0.5000000000000000 & 0.3888291333743084 & 0.0511249999999990\end{array}$

$\begin{array}{llll}0.2999840306611290 & 0.0555470190534706 & 0.0511249999999990\end{array}$

$\begin{array}{llll}0.8999520919833941 & 0.0555470190534706 & 0.0511249999999990\end{array}$

$\begin{array}{llll}0.8999520919833941 & 0.3888291333743084 & 0.0511249999999990\end{array}$

$\begin{array}{llll}0.7000159693388710 & 0.0555470190534706 & 0.0511249999999990\end{array}$ 
$\begin{array}{llll}0.5003708223114508 & 0.6117148774940682 & 0.1022251117228553\end{array}$

$\begin{array}{llll}0.3999864615072306 & 0.7778545521562902 & 0.1022613301188244\end{array}$

$\begin{array}{llll}0.6003254262286344 & 0.7773148543595999 & 0.1023185436290339\end{array}$

$\begin{array}{llll}0.7002589677452928 & 0.6099436863781633 & 0.1028366404573032\end{array}$

$0.3000079846912495 \quad 0.61156144110731350 .1021109743022565$

$\begin{array}{llll}0.1996932416443681 & 0.4437492400750833 & 0.1021033780021855\end{array}$

$\begin{array}{llll}0.8001453995583157 & 0.4445140947792382 & 0.1022087850195820\end{array}$

$\begin{array}{llll}0.4007498659710124 & 0.4449046380055967 & 0.1018444066616125\end{array}$

$\begin{array}{llll}0.6007024876927576 & 0.4445952239024634 & 0.1027651127101875\end{array}$

$\begin{array}{llll}0.9012269745611389 & 0.2769213758751529 & 0.1017132735206517\end{array}$

$\begin{array}{llll}0.1002306394846618 & 0.2772489262451748 & 0.1023066317635678\end{array}$

$\begin{array}{lllll}0.4999549514891871 & 0.9442762011134664 & 0.1018878921622689\end{array}$

$\begin{array}{llll}0.8010395387792507 & 0.1101498571673633 & 0.1018521188694061\end{array}$

$\begin{array}{llll}0.2009178022014480 & 0.1114277476466976 & 0.1030316234792690\end{array}$

$\begin{array}{llll}0.0004479975075419 & 0.1104586368267212 & 0.1021880572346737\end{array}$

$\begin{array}{llll}0.7007822787147617 & 0.9436700034372685 & 0.1020183875966741\end{array}$

$\begin{array}{llll}0.2993730471998298 & 0.9442585706452795 & 0.1021267777860961\end{array}$

$\begin{array}{llll}0.0998844642707187 & 0.9441515892127416 & 0.1022197439782013\end{array}$

$\begin{array}{llll}0.9003210442899645 & 0.9437937539285379 & 0.1022667108727314\end{array}$

$\begin{array}{llll}0.5997939185738442 & 0.1103579795291824 & 0.1018995351535954\end{array}$

$\begin{array}{llll}0.3984608992351147 & 0.1114221093403810 & 0.1027990415619234\end{array}$

$\begin{array}{llll}0.2997481700038720 & 0.2769523010726229 & 0.1028540394802777\end{array}$

$\begin{array}{llll}0.7004736624528439 & 0.2771957655913891 & 0.1015510673824424\end{array}$

$\begin{array}{llll}0.8002205760376768 & 0.7772791974403123 & 0.1022539445371281\end{array}$

$\begin{array}{lllll}1.0001492368689009 & 0.4440855185212867 & 0.1021010770351824\end{array}$

$\begin{array}{llll}0.1996662279365870 & 0.7776261889999538 & 0.1022564004250914\end{array}$

$\begin{array}{llll}1.0000032582391263 & 0.7774287687736413 & 0.1022818190917601\end{array}$

$\begin{array}{llll}0.4993029047037874 & 0.2760792334478078 & 0.1024034844994748\end{array}$

$\begin{array}{llll}0.9001410458322688 & 0.6107817194869399 & 0.1022704954362047\end{array}$

$\begin{array}{llll}0.0997399198132415 & 0.6108127509205771 & 0.1022202908016724\end{array}$

$\begin{array}{llll}0.6017768478204693 & 0.6674269240609796 & 0.1544456865909716\end{array}$

0.39878842767880630 .66795447490409620 .1541543214537593

$\begin{array}{llll}0.2976576339125949 & 0.5015373819705401 & 0.1527594770334402\end{array}$

$\begin{array}{llll}0.7042895495106628 & 0.4977079583665504 & 0.1556886297459665\end{array}$

$\begin{array}{llll}0.5005960801852710 & 0.5041247686206695 & 0.1530880330191857\end{array}$

$\begin{array}{llll}0.5001694004011453 & 0.8330941806512318 & 0.1541247175843094\end{array}$

$\begin{array}{llll}0.0984895652101586 & 0.1663232405429052 & 0.1542545646780425\end{array}$

$\begin{array}{llll}0.9007753996204753 & 0.1657158627580606 & 0.1545269889885640\end{array}$

$0.1994951463907718-0.00046304594884380 .1544600816290426$

$0.8007122584039704-0.00075813659570770 .1545834843584190$

$\begin{array}{llll}-0.0000015489008320 & -0.0004926814850207 & 0.1543745389558986\end{array}$

$\begin{array}{llll}-0.0001081098390195 & 0.3332285810804533 & 0.1544197409772299\end{array}$

$\begin{array}{llll}0.7007087605522159 & 0.8327084075437218 & 0.1546073805477957\end{array}$

$\begin{array}{llll}0.2994290210895148 & 0.8331281270484188 & 0.1545448163572448\end{array}$

$0.6021274956810577-0.00316213280914950 .1532572277213754$

$0.3984662414787434-0.00274067802601380 .1532452868895419$

$\begin{array}{llll}0.2982600700412699 & 0.1672641308416661 & 0.1575828468519264\end{array}$ 
$\begin{array}{llll}0.7031515351726777 & 0.1642368144259765 & 0.1527925936096060\end{array}$

$\begin{array}{lllll}0.1976878370257396 & 0.3345057563364656 & 0.1543152868000883\end{array}$

$\begin{array}{lllll}0.8033035408065641 & 0.3304880034565045 & 0.1524162346711441\end{array}$

$\begin{array}{lllll}0.9002152717217071 & 0.8331402342925197 & 0.1544963081950534\end{array}$

$\begin{array}{lllll}0.0996153460737634 & 0.8332784273980077 & 0.1544432179746846\end{array}$

$\begin{array}{lllll}0.5014881697023762 & 0.1589892573511338 & 0.1538247399702531\end{array}$

$\begin{array}{lllll}0.4005785683427698 & 0.3367489209385667 & 0.1522478423831378\end{array}$

$\begin{array}{lllll}0.6019547559249769 & 0.3283043625658474 & 0.1520526623974126\end{array}$

$\begin{array}{lllll}0.0994822230347046 & 0.5004500604652519 & 0.1545299571588045\end{array}$

$\begin{array}{lllll}0.8010107814284185 & 0.6665331848859961 & 0.1545058759070396\end{array}$

$\begin{array}{lllll}0.9020503665680322 & 0.4997462197360018 & 0.1542143635886959\end{array}$

$\begin{array}{llll}0.1991512009854153 & 0.6670994498159902 & 0.1544104474041664\end{array}$

$\begin{array}{lllll}0.9999763558457163 & 0.6667892865353302 & 0.1544794179574948\end{array}$

$\begin{array}{lllll}0.3116575059174874 & 0.2288018646745778 & 0.2084543766459591\end{array}$

$\begin{array}{lllll}0.5096184509318687 & 0.5492153205114966 & 0.2091065544964472\end{array}$

$\begin{array}{lllll}0.4976851566561665 & 0.1225961733929500 & 0.2100909000738448\end{array}$

$\begin{array}{lllll}0.6983005125657689 & 0.4503489414519064 & 0.2101446937408874\end{array}$

$\begin{array}{llll}0.4054658701499495 & 0.3926112683215539 & 0.2117465270241045\end{array}$

$\begin{array}{llll}0.5023642001996705 & 0.2296088344764113 & 0.2145329669459379\end{array}$

$\begin{array}{lllll}0.4008692052006992 & 0.2827770254203438 & 0.2119589470443872\end{array}$

$\begin{array}{lllll}0.6033337647917044 & 0.3910178092911478 & 0.2137458560399629\end{array}$

$\begin{array}{lllll}0.6009216660668608 & 0.2829948049557550 & 0.2154519812689210\end{array}$

$\begin{array}{lllll}0.5046175004324662 & 0.4492145102358702 & 0.2121126995754634\end{array}$

$\begin{array}{lllll}0.3310307601091776 & 0.4360319165015961 & 0.2130252697886223\end{array}$

$\begin{array}{lllll}0.6754697227404465 & 0.2393736742275286 & 0.2162797519191349\end{array}$

$\begin{array}{lllll}0.6913765095408806 & 0.5213398617989511 & 0.2207680831366951\end{array}$

$\begin{array}{lllll}0.7673782307877985 & 0.4138118517651034 & 0.2163799942290413\end{array}$

$\begin{array}{lllll}0.5612233143911398 & 0.0796502994930947 & 0.2173429954234406\end{array}$

$\begin{array}{llll}0.4247981065829583 & 0.0921215398909799 & 0.2157716718257293\end{array}$

Zwitterion on $\mathrm{Cu}$ - VASP - revPBE +D3-calculated lattice - ( $\mathrm{T}-0$ site) $\mathrm{Cu} \mathrm{O} \mathrm{N} \mathrm{C} \mathrm{H}$

1.00000000000000

$\begin{array}{llll}12.5239999999999991 & 0.0000000000000000 & 0.0000000000000000\end{array}$

$\begin{array}{llll}0.0000000000000000 & 13.0160000000000000 & 0.0000000000000000\end{array}$

$\begin{array}{llll}0.0000000000000000 & 0.0000000000000000 & 40.0000000000000000\end{array}$

$\begin{array}{ccccc}\mathrm{Cu} & \mathrm{O} & \mathrm{N} & \mathrm{C} & \mathrm{H}\end{array}$

$\begin{array}{lllll}120 & 2 & 2 & 6 & 6\end{array}$

Direct

$\begin{array}{llll}0.0000000000000000 & 0.6666410571604189 & 0.0000000000000000\end{array}$

$\begin{array}{llll}0.2000159693388710 & 0.6666410571604189 & 0.0000000000000000\end{array}$

$\begin{array}{lllll}0.3999520919833941 & 0.6666410571604189 & 0.0000000000000000\end{array}$

$\begin{array}{lllll}0.2999840306611290 & 0.8332821143208378 & 0.0000000000000000\end{array}$

$\begin{array}{lllll}0.0000000000000000 & 0.3332821143208378 & 0.0000000000000000\end{array}$

$\begin{array}{llll}0.2000159693388710 & 0.3332821143208378 & 0.0000000000000000\end{array}$

$\begin{array}{lllll}0.0999680613222580 & 0.4999231714812566 & 0.0000000000000000\end{array}$

$\begin{array}{llll}0.0999680613222580 & 0.8332821143208378 & 0.0000000000000000\end{array}$ 
$\begin{array}{lll}0.0000000000000000 & 0.0000000000000000 & 0.0000000000000000\end{array}$

$\begin{array}{llll}0.5999680613222580 & 0.6666410571604189 & 0.0000000000000000\end{array}$

$\begin{array}{llll}0.7999840306611290 & 0.6666410571604189 & 0.0000000000000000\end{array}$

$\begin{array}{llll}0.7000159693388710 & 0.8332821143208378 & 0.0000000000000000\end{array}$

$\begin{array}{llll}0.3999520919833941 & 0.3332821143208378 & 0.0000000000000000\end{array}$

$\begin{array}{llll}0.5999680613222580 & 0.3332821143208378 & 0.0000000000000000\end{array}$

$\begin{array}{llll}0.5000000000000000 & 0.4999231714812566 & 0.0000000000000000\end{array}$

$\begin{array}{llll}0.5000000000000000 & 0.8332821143208378 & 0.0000000000000000\end{array}$

$\begin{array}{llll}0.2000159693388710 & 0.0000000000000000 & 0.0000000000000000\end{array}$

$\begin{array}{llll}0.3999520919833941 & 0.0000000000000000 & 0.0000000000000000\end{array}$

$\begin{array}{llll}0.2999840306611290 & 0.1666410571604189 & 0.0000000000000000\end{array}$

$\begin{array}{lll}0.2999840306611290 & 0.4999231714812566 & 0.0000000000000000\end{array}$

$\begin{array}{llll}0.0999680613222580 & 0.1666410571604189 & 0.0000000000000000\end{array}$

$\begin{array}{llll}0.7999840306611290 & 0.3332821143208378 & 0.0000000000000000\end{array}$

$\begin{array}{llll}0.8999520919833941 & 0.4999231714812566 & 0.0000000000000000\end{array}$

$\begin{array}{llll}0.8999520919833941 & 0.8332821143208378 & 0.0000000000000000\end{array}$

$\begin{array}{llll}0.5999680613222580 & 0.0000000000000000 & 0.0000000000000000\end{array}$

$\begin{array}{lll}0.7999840306611290 & 0.0000000000000000 & 0.0000000000000000\end{array}$

$\begin{array}{llll}0.7000159693388710 & 0.1666410571604189 & 0.0000000000000000\end{array}$

$\begin{array}{llll}0.7000159693388710 & 0.4999231714812566 & 0.0000000000000000\end{array}$

$\begin{array}{llll}0.5000000000000000 & 0.1666410571604189 & 0.0000000000000000\end{array}$

$\begin{array}{llll}0.8999520919833941 & 0.1666410571604189 & 0.0000000000000000\end{array}$

$\begin{array}{llll}0.0000000000000000 & 0.8888291333743084 & 0.0511249999999990\end{array}$

$\begin{array}{llll}0.2000159693388710 & 0.8888291333743084 & 0.0511249999999990\end{array}$

$\begin{array}{llll}0.3999520919833941 & 0.8888291333743084 & 0.0511249999999990\end{array}$

$\begin{array}{llll}0.0000000000000000 & 0.5554701905347272 & 0.0511249999999990\end{array}$

$\begin{array}{llll}0.2000159693388710 & 0.5554701905347272 & 0.0511249999999990\end{array}$

$\begin{array}{llll}0.0999680613222580 & 0.7221880762138895 & 0.0511249999999990\end{array}$

$\begin{array}{llll}0.0000000000000000 & 0.2221880762138895 & 0.0511249999999990\end{array}$

$\begin{array}{llll}0.5999680613222580 & 0.8888291333743084 & 0.0511249999999990\end{array}$

$\begin{array}{lllll}0.7999840306611290 & 0.8888291333743084 & 0.0511249999999990\end{array}$

$\begin{array}{llll}0.3999520919833941 & 0.5554701905347272 & 0.0511249999999990\end{array}$

$\begin{array}{llll}0.5999680613222580 & 0.5554701905347272 & 0.0511249999999990\end{array}$

$\begin{array}{llll}0.5000000000000000 & 0.7221880762138895 & 0.0511249999999990\end{array}$

$\begin{array}{llll}0.2000159693388710 & 0.2221880762138895 & 0.0511249999999990\end{array}$

$\begin{array}{llll}0.3999520919833941 & 0.2221880762138895 & 0.0511249999999990\end{array}$

$\begin{array}{llll}0.2999840306611290 & 0.3888291333743084 & 0.0511249999999990\end{array}$

$\begin{array}{lll}0.2999840306611290 & 0.7221880762138895 & 0.0511249999999990\end{array}$

$\begin{array}{llll}0.0999680613222580 & 0.0555470190534706 & 0.0511249999999990\end{array}$

$\begin{array}{llll}0.0999680613222580 & 0.3888291333743084 & 0.0511249999999990\end{array}$

$\begin{array}{llll}0.7999840306611290 & 0.5554701905347272 & 0.0511249999999990\end{array}$

$\begin{array}{llll}0.8999520919833941 & 0.7221880762138895 & 0.0511249999999990\end{array}$

$\begin{array}{llll}0.5999680613222580 & 0.2221880762138895 & 0.0511249999999990\end{array}$

$\begin{array}{llll}0.7999840306611290 & 0.2221880762138895 & 0.0511249999999990\end{array}$

$\begin{array}{llll}0.7000159693388710 & 0.3888291333743084 & 0.0511249999999990\end{array}$

$\begin{array}{llll}0.7000159693388710 & 0.7221880762138895 & 0.0511249999999990\end{array}$

$\begin{array}{llll}0.5000000000000000 & 0.0555470190534706 & 0.0511249999999990\end{array}$ 
$\begin{array}{llll}0.5000000000000000 & 0.3888291333743084 & 0.0511249999999990\end{array}$

$\begin{array}{llll}0.2999840306611290 & 0.0555470190534706 & 0.0511249999999990\end{array}$

$\begin{array}{llll}0.8999520919833941 & 0.0555470190534706 & 0.0511249999999990\end{array}$

$\begin{array}{llll}0.8999520919833941 & 0.3888291333743084 & 0.0511249999999990\end{array}$

$\begin{array}{llll}0.7000159693388710 & 0.0555470190534706 & 0.0511249999999990\end{array}$

$\begin{array}{llll}0.4994686768257473 & 0.6118006322301899 & 0.1017591765977110\end{array}$

$\begin{array}{llll}0.3996858234770061 & 0.7783765616315628 & 0.1022624760418191\end{array}$

$\begin{array}{llll}0.6000479045871688 & 0.7786355504030860 & 0.1021065169757534\end{array}$

$\begin{array}{llll}0.7004954962866714 & 0.6117795960816262 & 0.1021054988112051\end{array}$

0.29931606795181450 .61182496518675030 .1020590354712141

$\begin{array}{llll}0.1986874691069176 & 0.4439760287452144 & 0.1018227146390448\end{array}$

$\begin{array}{llll}0.8011435180075380 & 0.4441400946848554 & 0.1019523419444832\end{array}$

$\begin{array}{llll}0.3994183481991474 & 0.4443580581522981 & 0.1016668655866527\end{array}$

$\begin{array}{lll}0.6007257389695946 & 0.4445390304348494 & 0.1017733163259418\end{array}$

$\begin{array}{llll}0.9004975650097434 & 0.2773528833459918 & 0.1022594112020418\end{array}$

$\begin{array}{llll}0.0994621030795780 & 0.2773253336796904 & 0.1022636975994520\end{array}$

$\begin{array}{llll}0.4999138143466402 & 0.9449215400190436 & 0.1022713548939338\end{array}$

$\begin{array}{llll}0.8002003250255463 & 0.1109060413293737 & 0.1023848565305290\end{array}$

$\begin{array}{llll}0.1997766297374652 & 0.1108911887331851 & 0.1023787424177153\end{array}$

$\begin{array}{llll}-0.0000067732586053 & 0.1108023796497023 & 0.1024202227601940\end{array}$

$\begin{array}{llll}0.7001874637306778 & 0.9447982494894324 & 0.1022881190307287\end{array}$

$\begin{array}{llll}0.2997449870240932 & 0.9447740437552836 & 0.1022925452442180\end{array}$

$\begin{array}{llll}0.0998020065747025 & 0.9441874064827352 & 0.1023552117767866\end{array}$

$\begin{array}{llll}0.9001069521780920 & 0.9441461103413578 & 0.1023591128271237\end{array}$

$\begin{array}{llll}0.6001238489705613 & 0.1107858571580716 & 0.1023165685887456\end{array}$

$\begin{array}{llll}0.3998700619858341 & 0.1108098521985060 & 0.1023167626260481\end{array}$

$\begin{array}{llll}0.2992235596454850 & 0.2769690336581278 & 0.1019915766463184\end{array}$

$\begin{array}{llll}0.7007636853319255 & 0.2770007286572580 & 0.1019805252383496\end{array}$

$\begin{array}{llll}0.8002531594910721 & 0.7774968518328785 & 0.1025924054278979\end{array}$

$\begin{array}{llll}0.9999363319200202 & 0.4441551174013196 & 0.1022786475759852\end{array}$

$\begin{array}{llll}0.1995858201981423 & 0.7776185190112813 & 0.1025403603043246\end{array}$

0.99989506245564820 .77746565115666550 .1022907774551336

$\begin{array}{llll}0.5000242696419638 & 0.2768647671983976 & 0.1016175804027803\end{array}$

$\begin{array}{llll}0.8997646603426221 & 0.6110684280553194 & 0.1025967548325602\end{array}$

$\begin{array}{llll}0.0998436444485431 & 0.6109668908200998 & 0.1025116022678024\end{array}$

$\begin{array}{llll}0.5989045486322326 & 0.6684906216249810 & 0.1525920743960938\end{array}$

$\begin{array}{llll}0.3986002756507628 & 0.6681881477373378 & 0.1533587938714947\end{array}$

$\begin{array}{lll}0.2976105428982560 & 0.4990434461298095 & 0.1519183384863273\end{array}$

$\begin{array}{llll}0.7022240684781574 & 0.4997482670535449 & 0.1523764776798134\end{array}$

$\begin{array}{llll}0.4997735014804244 & 0.4994863424116037 & 0.1518306667723273\end{array}$

$\begin{array}{llll}0.4996451509768620 & 0.8347112411505982 & 0.1542009573211737\end{array}$

$\begin{array}{llll}0.0999329487621522 & 0.1668064824671178 & 0.1546545328617905\end{array}$

$\begin{array}{llll}0.8999974941616878 & 0.1668026484986155 & 0.1546559248981862\end{array}$

$\begin{array}{llll}0.1998177945723469 & 0.0003617705184700 & 0.1545319492613291\end{array}$

$\begin{array}{lllll}0.8000475711066511 & 0.0003072484640584 & 0.1545283913762268\end{array}$

$\begin{array}{llll}-0.0000572682136271 & 0.0001828994544233 & 0.1545378748167684\end{array}$

$\begin{array}{llll}-0.0000318996072834 & 0.3332245104706742 & 0.1548536497085563\end{array}$ 
$\begin{array}{llll}0.6997671130513071 & 0.8340983597505467 & 0.1542727888046113\end{array}$

$\begin{array}{llll}0.2996639411673176 & 0.8342980039728976 & 0.1543043377292832\end{array}$

$\begin{array}{llll}0.5999899256485174 & 0.0004259446094675 & 0.1545140653122374\end{array}$

$\begin{array}{llll}0.3999099838262533 & 0.0005600991009727 & 0.1544970209796075\end{array}$

$\begin{array}{llll}0.3000078247366278 & 0.1670275652660981 & 0.1548079768099519\end{array}$

$\begin{array}{llll}0.6999608824687845 & 0.1669976726719775 & 0.1547966405353019\end{array}$

$\begin{array}{llll}0.1995492273656692 & 0.3328017560240900 & 0.1540740870621170\end{array}$

$\begin{array}{lllll}0.8004034283748969 & 0.3328269550220821 & 0.1540087688559486\end{array}$

$\begin{array}{llll}0.9000717355754326 & 0.8335062548035533 & 0.1544799937785536\end{array}$

$\begin{array}{llll}0.0995180821218464 & 0.8335410700472815 & 0.1544717215963784\end{array}$

$\begin{array}{llll}0.5000612886121546 & 0.1666830517166289 & 0.1545264093848245\end{array}$

$\begin{array}{llll}0.4000948041323075 & 0.3319369058757273 & 0.1524952411142714\end{array}$

$\begin{array}{llll}0.5999024800174918 & 0.3321311291385365 & 0.1524572314721246\end{array}$

$\begin{array}{llll}0.0994024956650856 & 0.4992862288617189 & 0.1546960442229625\end{array}$

$\begin{array}{llll}0.7998524497789595 & 0.6668406578908955 & 0.1555115661640597\end{array}$

$\begin{array}{llll}0.9006951057226303 & 0.4993137456354095 & 0.1546869764612201\end{array}$

$\begin{array}{llll}0.1989562810924145 & 0.6665568522534373 & 0.1551243118738518\end{array}$

$\begin{array}{llll}0.9997571734734390 & 0.6664976068830081 & 0.1546253825720502\end{array}$

0.31513448246559240 .61069817735139090 .2099435899056032

$\begin{array}{llll}0.6998338182343895 & 0.5969835625306255 & 0.2099293649063579\end{array}$

$\begin{array}{llll}0.3058342681811093 & 0.4046319330524185 & 0.2220896741956939\end{array}$

$\begin{array}{llll}0.6948267287859970 & 0.3922663939717590 & 0.2222273287453573\end{array}$

$\begin{array}{llll}0.5077929198413397 & 0.6108015891700137 & 0.2124620241322057\end{array}$

$\begin{array}{llll}0.4038561579167829 & 0.4498477696084828 & 0.2196615032056136\end{array}$

$\begin{array}{llll}0.4049904864879632 & 0.5627097536473286 & 0.2144415098417654\end{array}$

$\begin{array}{llll}0.5999506918844093 & 0.4433215538298139 & 0.2197270357098619\end{array}$

$\begin{array}{llll}0.5000387526731778 & 0.3934588040486320 & 0.2207071875308013\end{array}$

$\begin{array}{llll}0.6066120390140836 & 0.5557442349959252 & 0.2144568215730917\end{array}$

$0.51078026711344550 .6947146590150794 \quad 0.2116381480814699$

$\begin{array}{llll}0.4969821878287014 & 0.3096048302833396 & 0.2218183855657433\end{array}$

$\begin{array}{llll}0.7607748687434467 & 0.4343239110734651 & 0.2157580834472575\end{array}$

$\begin{array}{llll}0.6981571165763114 & 0.3162535277740391 & 0.2160446034929519\end{array}$

$\begin{array}{llll}0.2980247905282949 & 0.3288108200884202 & 0.2160472930987493\end{array}$

$\begin{array}{llll}0.2423632114992224 & 0.4498823199619940 & 0.2155426823202212\end{array}$

Zwitterion on $\mathrm{Cu}$ - VASP - revPBE+D3-calculated lattice - (T - 30 site)

$\mathrm{Cu} \mathrm{O} \mathrm{N} \mathrm{C} \mathrm{H}$

1.00000000000000

$\begin{array}{lll}12.5239999999999991 & 0.0000000000000000 & 0.0000000000000000\end{array}$

$\begin{array}{llll}0.0000000000000000 & 13.0160000000000000 & 0.0000000000000000\end{array}$

$\begin{array}{llll}0.0000000000000000 & 0.0000000000000000 & 40.0000000000000000\end{array}$

$\begin{array}{lllll}\mathrm{Cu} & \mathrm{O} & \mathrm{N} & \mathrm{C} & \mathrm{H}\end{array}$

$\begin{array}{lllll}120 & 2 & 2 & 6 & 6\end{array}$

Direct

$\begin{array}{llll}0.0000000000000000 & 0.6666410571604189 & 0.0000000000000000\end{array}$

$\begin{array}{llll}0.2000159693388710 & 0.6666410571604189 & 0.0000000000000000\end{array}$

$\begin{array}{llll}0.3999520919833941 & 0.6666410571604189 & 0.0000000000000000\end{array}$ 
$\begin{array}{lll}0.2999840306611290 & 0.8332821143208378 & 0.0000000000000000\end{array}$

$\begin{array}{llll}0.0000000000000000 & 0.3332821143208378 & 0.0000000000000000\end{array}$

$\begin{array}{llll}0.2000159693388710 & 0.3332821143208378 & 0.0000000000000000\end{array}$

$\begin{array}{llll}0.0999680613222580 & 0.4999231714812566 & 0.0000000000000000\end{array}$

$\begin{array}{lll}0.0999680613222580 & 0.8332821143208378 & 0.0000000000000000\end{array}$

$\begin{array}{lllll}0.0000000000000000 & 0.0000000000000000 & 0.0000000000000000\end{array}$

$\begin{array}{llll}0.5999680613222580 & 0.6666410571604189 & 0.0000000000000000\end{array}$

$\begin{array}{llll}0.7999840306611290 & 0.6666410571604189 & 0.0000000000000000\end{array}$

$\begin{array}{llll}0.7000159693388710 & 0.8332821143208378 & 0.0000000000000000\end{array}$

$\begin{array}{llll}0.3999520919833941 & 0.3332821143208378 & 0.0000000000000000\end{array}$

$\begin{array}{llll}0.5999680613222580 & 0.3332821143208378 & 0.0000000000000000\end{array}$

$\begin{array}{llll}0.5000000000000000 & 0.4999231714812566 & 0.0000000000000000\end{array}$

$\begin{array}{llll}0.5000000000000000 & 0.8332821143208378 & 0.0000000000000000\end{array}$

$\begin{array}{llll}0.2000159693388710 & 0.0000000000000000 & 0.0000000000000000\end{array}$

$\begin{array}{llll}0.3999520919833941 & 0.0000000000000000 & 0.0000000000000000\end{array}$

$\begin{array}{llll}0.2999840306611290 & 0.1666410571604189 & 0.0000000000000000\end{array}$

$\begin{array}{llll}0.2999840306611290 & 0.4999231714812566 & 0.0000000000000000\end{array}$

$\begin{array}{llll}0.0999680613222580 & 0.1666410571604189 & 0.0000000000000000\end{array}$

$\begin{array}{llll}0.7999840306611290 & 0.3332821143208378 & 0.0000000000000000\end{array}$

$\begin{array}{llll}0.8999520919833941 & 0.4999231714812566 & 0.0000000000000000\end{array}$

$\begin{array}{llll}0.8999520919833941 & 0.8332821143208378 & 0.0000000000000000\end{array}$

$\begin{array}{llll}0.5999680613222580 & 0.0000000000000000 & 0.0000000000000000\end{array}$

$\begin{array}{lll}0.7999840306611290 & 0.0000000000000000 & 0.0000000000000000\end{array}$

$\begin{array}{llll}0.7000159693388710 & 0.1666410571604189 & 0.0000000000000000\end{array}$

$\begin{array}{llll}0.7000159693388710 & 0.4999231714812566 & 0.0000000000000000\end{array}$

$\begin{array}{llll}0.5000000000000000 & 0.1666410571604189 & 0.0000000000000000\end{array}$

$\begin{array}{llll}0.8999520919833941 & 0.1666410571604189 & 0.0000000000000000\end{array}$

$\begin{array}{llll}0.0000000000000000 & 0.8888291333743084 & 0.0511249999999990\end{array}$

$\begin{array}{llll}0.2000159693388710 & 0.8888291333743084 & 0.0511249999999990\end{array}$

$\begin{array}{llll}0.3999520919833941 & 0.8888291333743084 & 0.0511249999999990\end{array}$

$\begin{array}{llll}0.0000000000000000 & 0.5554701905347272 & 0.0511249999999990\end{array}$

$\begin{array}{llll}0.2000159693388710 & 0.5554701905347272 & 0.0511249999999990\end{array}$

$\begin{array}{llll}0.0999680613222580 & 0.7221880762138895 & 0.0511249999999990\end{array}$

$\begin{array}{llll}0.0000000000000000 & 0.2221880762138895 & 0.0511249999999990\end{array}$

$\begin{array}{llll}0.5999680613222580 & 0.8888291333743084 & 0.0511249999999990\end{array}$

$\begin{array}{llll}0.7999840306611290 & 0.8888291333743084 & 0.0511249999999990\end{array}$

$\begin{array}{llll}0.3999520919833941 & 0.5554701905347272 & 0.0511249999999990\end{array}$

$\begin{array}{llll}0.5999680613222580 & 0.5554701905347272 & 0.0511249999999990\end{array}$

$\begin{array}{llll}0.5000000000000000 & 0.7221880762138895 & 0.0511249999999990\end{array}$

$\begin{array}{llll}0.2000159693388710 & 0.2221880762138895 & 0.0511249999999990\end{array}$

$\begin{array}{llll}0.3999520919833941 & 0.2221880762138895 & 0.0511249999999990\end{array}$

$\begin{array}{llll}0.2999840306611290 & 0.3888291333743084 & 0.0511249999999990\end{array}$

$0.2999840306611290 \quad 0.72218807621388950 .0511249999999990$

$\begin{array}{llll}0.0999680613222580 & 0.0555470190534706 & 0.0511249999999990\end{array}$

$\begin{array}{llll}0.0999680613222580 & 0.3888291333743084 & 0.0511249999999990\end{array}$

$\begin{array}{llll}0.7999840306611290 & 0.5554701905347272 & 0.0511249999999990\end{array}$

$\begin{array}{llll}0.8999520919833941 & 0.7221880762138895 & 0.0511249999999990\end{array}$ 
$\begin{array}{lll}0.5999680613222580 & 0.2221880762138895 & 0.0511249999999990\end{array}$

$\begin{array}{llll}0.7999840306611290 & 0.2221880762138895 & 0.0511249999999990\end{array}$

$\begin{array}{llll}0.7000159693388710 & 0.3888291333743084 & 0.0511249999999990\end{array}$

$\begin{array}{llll}0.7000159693388710 & 0.7221880762138895 & 0.0511249999999990\end{array}$

$\begin{array}{llll}0.5000000000000000 & 0.0555470190534706 & 0.0511249999999990\end{array}$

$\begin{array}{llll}0.5000000000000000 & 0.3888291333743084 & 0.0511249999999990\end{array}$

$\begin{array}{llll}0.2999840306611290 & 0.0555470190534706 & 0.0511249999999990\end{array}$

$\begin{array}{llll}0.8999520919833941 & 0.0555470190534706 & 0.0511249999999990\end{array}$

$\begin{array}{llll}0.8999520919833941 & 0.3888291333743084 & 0.0511249999999990\end{array}$

$\begin{array}{llll}0.7000159693388710 & 0.0555470190534706 & 0.0511249999999990\end{array}$

$\begin{array}{llll}0.5012611852423318 & 0.6121468997966959 & 0.1022071092608675\end{array}$

$\begin{array}{llll}0.4001299201622154 & 0.7779524703487476 & 0.1021706663101526\end{array}$

$\begin{array}{llll}0.6002964026800075 & 0.7777162487846160 & 0.1025387393225609\end{array}$

$\begin{array}{llll}0.6999608263421540 & 0.6109724307948785 & 0.1028411232780893\end{array}$

$\begin{array}{llll}0.2997553002075218 & 0.6100064136144849 & 0.1025204856149023\end{array}$

$\begin{array}{llll}0.1996445425263099 & 0.4443759055448882 & 0.1023191045376097\end{array}$

$\begin{array}{llll}0.8005074856038125 & 0.4440652053349756 & 0.1020122113706160\end{array}$

$\begin{array}{llll}0.3982622881142756 & 0.4436024449884747 & 0.1028413948334972\end{array}$

$\begin{array}{llll}0.6012432570344183 & 0.4448764940241671 & 0.1022124771018568\end{array}$

$\begin{array}{llll}0.9004253819380633 & 0.2770519858440982 & 0.1019867583155738\end{array}$

$\begin{array}{llll}0.0994769532078115 & 0.2769905005044609 & 0.1020753157295747\end{array}$

$\begin{array}{llll}0.4999969873764936 & 0.9445309601426917 & 0.1022285171173998\end{array}$

$\begin{array}{llll}0.8001633578637076 & 0.1107754716743236 & 0.1022681343759724\end{array}$

$\begin{array}{llll}0.1994046863488267 & 0.1104715860054792 & 0.1021543153442886\end{array}$

$\begin{array}{llll}-0.0002840382279263 & 0.1105534376087442 & 0.1022674111283590\end{array}$

$\begin{array}{llll}0.6999522884919852 & 0.9447472079908714 & 0.1022200895232456\end{array}$

$\begin{array}{llll}0.2998587289674695 & 0.9442887741911140 & 0.1022619742787390\end{array}$

$\begin{array}{llll}0.0999493068121479 & 0.9440651790519351 & 0.1022740655209706\end{array}$

$\begin{array}{llll}0.9002165586940660 & 0.9442746279539735 & 0.1022484350099008\end{array}$

$\begin{array}{llll}0.6001924681095098 & 0.1109264127827176 & 0.1021089084116646\end{array}$

$\begin{array}{llll}0.3997979123948597 & 0.1106236749941013 & 0.1021509608081512\end{array}$

$\begin{array}{llll}0.2994911961449877 & 0.2771944188126124 & 0.1021385635076593\end{array}$

$\begin{array}{llll}0.7002557552714490 & 0.2771352841016506 & 0.1018529369756896\end{array}$

$\begin{array}{llll}0.8001966171541812 & 0.7779453509594135 & 0.1021524055933635\end{array}$

$\begin{array}{llll}1.0001042896915611 & 0.4438686939213970 & 0.1020145374947150\end{array}$

$\begin{array}{llll}0.1998665698861866 & 0.7775454027135733 & 0.1022496451411100\end{array}$

$\begin{array}{llll}1.0000722533955746 & 0.7775625476545135 & 0.1022166717846818\end{array}$

$\begin{array}{llll}0.4987425844699207 & 0.2776508963700591 & 0.1021931444840436\end{array}$

$\begin{array}{llll}0.9000107459045428 & 0.6108506044673740 & 0.1020196322116618\end{array}$

$\begin{array}{llll}0.0999453862670585 & 0.6109669175080209 & 0.1022239377311657\end{array}$

$\begin{array}{lllll}0.6046170578284038 & 0.6706653780947269 & 0.1552024686412457\end{array}$

$\begin{array}{llll}0.3999196004463436 & 0.6676852040564685 & 0.1527273212031620\end{array}$

$\begin{array}{llll}0.2932746674314073 & 0.4989805043893761 & 0.1553011207708823\end{array}$

$\begin{array}{llll}0.7057640636297544 & 0.4983940000152362 & 0.1543906059308004\end{array}$

$\begin{array}{llll}0.4998331060085285 & 0.5001699183181357 & 0.1514515910671396\end{array}$

$\begin{array}{llll}0.4996730120199327 & 0.8345691156206141 & 0.1542044169789500\end{array}$

$\begin{array}{llll}0.0991383890626209 & 0.1657572489641548 & 0.1545538226633164\end{array}$ 
$\begin{array}{lll}0.9001220058321781 & 0.1661490468661706 & 0.1545343516938611\end{array}$

$0.1995175692368249-0.00071521977975590 .1545898063133598$

0.80043278006525730 .00070290404120190 .1544667453432454

$-0.0000093334689183-0.00006175026854190 .1544601080453971$

$\begin{array}{llll}-0.0010304041623997 & 0.3325376644503468 & 0.1544222515729569\end{array}$

$\begin{array}{llll}0.7018948457829758 & 0.8360485135055702 & 0.1539700912450270\end{array}$

$\begin{array}{llll}0.2999536711915652 & 0.8333791675854620 & 0.1543512788647314\end{array}$

$\begin{array}{llll}0.6000339530920786 & 0.0002191165800632 & 0.1544274778522508\end{array}$

$0.3999062975156579-0.00021148235023560 .1545420251525413$

0.29837241192324580 .16373442265292760 .1538306487799626

$\begin{array}{lllll}0.6998498258906254 & 0.1666290675649824 & 0.1545510362222524\end{array}$

$\begin{array}{llll}0.1965998745928088 & 0.3311849852352149 & 0.1533489913469451\end{array}$

$\begin{array}{llll}0.8009376342390004 & 0.3308966142818097 & 0.1535190861351367\end{array}$

$\begin{array}{llll}0.9009828273962220 & 0.8341270626356344 & 0.1545278298601951\end{array}$

$\begin{array}{llll}0.0998263263973316 & 0.8336067342921801 & 0.1543917852820418\end{array}$

$\begin{array}{llll}0.5005502692467483 & 0.1645959192923671 & 0.1538855064361392\end{array}$

$\begin{array}{llll}0.3968564777374150 & 0.3279261670006788 & 0.1544806720344092\end{array}$

$\begin{array}{llll}0.6003311785445080 & 0.3316733352553231 & 0.1523965767624673\end{array}$

$\begin{array}{llll}0.0968968950804954 & 0.4999172367089742 & 0.1541154891360703\end{array}$

$\begin{array}{llll}0.8035590928783880 & 0.6681281973353717 & 0.1537188727922385\end{array}$

$\begin{array}{llll}0.9020269278107146 & 0.4996236908865228 & 0.1538174485474367\end{array}$

$\begin{array}{llll}0.1988962276757805 & 0.6678968140829314 & 0.1542494184103797\end{array}$

$\begin{array}{llll}1.0002722286763122 & 0.6670264438088434 & 0.1545312983376025\end{array}$

$\begin{array}{llll}0.2841357371101772 & 0.4984878184334081 & 0.2081146197865271\end{array}$

$\begin{array}{llll}0.6210682878142110 & 0.6768137771493745 & 0.2086558109601015\end{array}$

$\begin{array}{llll}0.3857152549461485 & 0.3154479336157512 & 0.2099655344236608\end{array}$

$\begin{array}{llll}0.7268069854384415 & 0.4956753950455549 & 0.2099407770899294\end{array}$

$\begin{array}{llll}0.4492201179977127 & 0.5933029693799803 & 0.2134523798691423\end{array}$

$\begin{array}{llll}0.4458602098074332 & 0.4047517884582335 & 0.2165817756866689\end{array}$

$\begin{array}{llll}0.3880344193899977 & 0.5008299026378255 & 0.2135793386764753\end{array}$

$\begin{array}{llll}0.6164534197439377 & 0.4946810453755727 & 0.2167028509749720\end{array}$

$\begin{array}{llll}0.5579342074705215 & 0.4025662894391059 & 0.2184348879443440\end{array}$

$\begin{array}{llll}0.5633344862845630 & 0.5934218143329010 & 0.2138027739280146\end{array}$

$\begin{array}{llll}0.4073058343263071 & 0.6670736781864618 & 0.2131784079203080\end{array}$

$\begin{array}{llll}0.5997636339190874 & 0.3287663504626658 & 0.2183270697651851\end{array}$

$\begin{array}{llll}0.7628028402394321 & 0.5635731200096631 & 0.2159242930145058\end{array}$

$\begin{array}{llll}0.7697248151365244 & 0.4324731056549609 & 0.2164444328024136\end{array}$

$\begin{array}{llll}0.4173919593540277 & 0.2469864296854977 & 0.2171006656143044\end{array}$

$\begin{array}{llll}0.3066904671605659 & 0.3233124746951486 & 0.2156619151021387\end{array}$

Zwitterion on $\mathrm{Cu}$ - VASP - revPBE+D3-calculated lattice - ( $\mathrm{T}$ - 60 site)

$\mathrm{Cu} \mathrm{O} \mathrm{N} \mathrm{C} \mathrm{H}$

1.00000000000000

$\begin{array}{llll}12.5239999999999991 & 0.0000000000000000 & 0.0000000000000000\end{array}$

$\begin{array}{llll}0.0000000000000000 & 13.0160000000000000 & 0.0000000000000000\end{array}$

$\begin{array}{llll}0.0000000000000000 & 0.0000000000000000 & 40.0000000000000000\end{array}$

$\mathrm{Cu} \quad \mathrm{O} \quad \mathrm{N} \quad \mathrm{C} \quad \mathrm{H}$ 
$\begin{array}{lllll}120 & 2 & 2 & 6 & 6\end{array}$

Direct

$0.0000000000000000 \quad 0.66664105716041890 .0000000000000000$

$\begin{array}{lll}0.2000159693388710 & 0.6666410571604189 & 0.0000000000000000\end{array}$

$\begin{array}{llll}0.3999520919833941 & 0.6666410571604189 & 0.0000000000000000\end{array}$

$\begin{array}{llll}0.2999840306611290 & 0.8332821143208378 & 0.0000000000000000\end{array}$

$\begin{array}{llll}0.0000000000000000 & 0.3332821143208378 & 0.0000000000000000\end{array}$

$\begin{array}{llll}0.2000159693388710 & 0.3332821143208378 & 0.0000000000000000\end{array}$

$\begin{array}{llll}0.0999680613222580 & 0.4999231714812566 & 0.0000000000000000\end{array}$

$\begin{array}{lll}0.0999680613222580 & 0.8332821143208378 & 0.0000000000000000\end{array}$

$\begin{array}{lllll}0.0000000000000000 & 0.0000000000000000 & 0.0000000000000000\end{array}$

$\begin{array}{llll}0.5999680613222580 & 0.6666410571604189 & 0.0000000000000000\end{array}$

$\begin{array}{llll}0.7999840306611290 & 0.6666410571604189 & 0.0000000000000000\end{array}$

$\begin{array}{llll}0.7000159693388710 & 0.8332821143208378 & 0.0000000000000000\end{array}$

$\begin{array}{llll}0.3999520919833941 & 0.3332821143208378 & 0.0000000000000000\end{array}$

$\begin{array}{llll}0.5999680613222580 & 0.3332821143208378 & 0.0000000000000000\end{array}$

$\begin{array}{llll}0.5000000000000000 & 0.4999231714812566 & 0.0000000000000000\end{array}$

$\begin{array}{llll}0.5000000000000000 & 0.8332821143208378 & 0.0000000000000000\end{array}$

$\begin{array}{llll}0.2000159693388710 & 0.0000000000000000 & 0.0000000000000000\end{array}$

$\begin{array}{llll}0.3999520919833941 & 0.0000000000000000 & 0.0000000000000000\end{array}$

$\begin{array}{llll}0.2999840306611290 & 0.1666410571604189 & 0.0000000000000000\end{array}$

$\begin{array}{llll}0.2999840306611290 & 0.4999231714812566 & 0.0000000000000000\end{array}$

$\begin{array}{llll}0.0999680613222580 & 0.1666410571604189 & 0.0000000000000000\end{array}$

$\begin{array}{llll}0.7999840306611290 & 0.3332821143208378 & 0.0000000000000000\end{array}$

$\begin{array}{llll}0.8999520919833941 & 0.4999231714812566 & 0.0000000000000000\end{array}$

$\begin{array}{llll}0.8999520919833941 & 0.8332821143208378 & 0.0000000000000000\end{array}$

$\begin{array}{llll}0.5999680613222580 & 0.0000000000000000 & 0.0000000000000000\end{array}$

$\begin{array}{lll}0.7999840306611290 & 0.0000000000000000 & 0.0000000000000000\end{array}$

$\begin{array}{llll}0.7000159693388710 & 0.1666410571604189 & 0.0000000000000000\end{array}$

$\begin{array}{llll}0.7000159693388710 & 0.4999231714812566 & 0.0000000000000000\end{array}$

$\begin{array}{lllll}0.5000000000000000 & 0.1666410571604189 & 0.0000000000000000\end{array}$

$\begin{array}{llll}0.8999520919833941 & 0.1666410571604189 & 0.0000000000000000\end{array}$

$\begin{array}{llll}0.0000000000000000 & 0.8888291333743084 & 0.0511249999999990\end{array}$

$\begin{array}{llll}0.2000159693388710 & 0.8888291333743084 & 0.0511249999999990\end{array}$

$\begin{array}{lllll}0.3999520919833941 & 0.8888291333743084 & 0.0511249999999990\end{array}$

$\begin{array}{llll}0.0000000000000000 & 0.5554701905347272 & 0.0511249999999990\end{array}$

$\begin{array}{llll}0.2000159693388710 & 0.5554701905347272 & 0.0511249999999990\end{array}$

$\begin{array}{llll}0.0999680613222580 & 0.7221880762138895 & 0.0511249999999990\end{array}$

$\begin{array}{llll}0.0000000000000000 & 0.2221880762138895 & 0.0511249999999990\end{array}$

$\begin{array}{llll}0.5999680613222580 & 0.8888291333743084 & 0.0511249999999990\end{array}$

$\begin{array}{llll}0.7999840306611290 & 0.8888291333743084 & 0.0511249999999990\end{array}$

$\begin{array}{llll}0.3999520919833941 & 0.5554701905347272 & 0.0511249999999990\end{array}$

$\begin{array}{llll}0.5999680613222580 & 0.5554701905347272 & 0.0511249999999990\end{array}$

$\begin{array}{llll}0.5000000000000000 & 0.7221880762138895 & 0.0511249999999990\end{array}$

$\begin{array}{llll}0.2000159693388710 & 0.2221880762138895 & 0.0511249999999990\end{array}$

$\begin{array}{llll}0.3999520919833941 & 0.2221880762138895 & 0.0511249999999990\end{array}$

$\begin{array}{llll}0.2999840306611290 & 0.3888291333743084 & 0.0511249999999990\end{array}$ 
$\begin{array}{lll}0.2999840306611290 & 0.7221880762138895 & 0.0511249999999990\end{array}$

$\begin{array}{llll}0.0999680613222580 & 0.0555470190534706 & 0.0511249999999990\end{array}$

$\begin{array}{llll}0.0999680613222580 & 0.3888291333743084 & 0.0511249999999990\end{array}$

$\begin{array}{llll}0.7999840306611290 & 0.5554701905347272 & 0.0511249999999990\end{array}$

$\begin{array}{llll}0.8999520919833941 & 0.7221880762138895 & 0.0511249999999990\end{array}$

$\begin{array}{llll}0.5999680613222580 & 0.2221880762138895 & 0.0511249999999990\end{array}$

$\begin{array}{llll}0.7999840306611290 & 0.2221880762138895 & 0.0511249999999990\end{array}$

$\begin{array}{llll}0.7000159693388710 & 0.3888291333743084 & 0.0511249999999990\end{array}$

$\begin{array}{llll}0.7000159693388710 & 0.7221880762138895 & 0.0511249999999990\end{array}$

$\begin{array}{llll}0.5000000000000000 & 0.0555470190534706 & 0.0511249999999990\end{array}$

$\begin{array}{llll}0.5000000000000000 & 0.3888291333743084 & 0.0511249999999990\end{array}$

$\begin{array}{llll}0.2999840306611290 & 0.0555470190534706 & 0.0511249999999990\end{array}$

$\begin{array}{llll}0.8999520919833941 & 0.0555470190534706 & 0.0511249999999990\end{array}$

$\begin{array}{llll}0.8999520919833941 & 0.3888291333743084 & 0.0511249999999990\end{array}$

$\begin{array}{llll}0.7000159693388710 & 0.0555470190534706 & 0.0511249999999990\end{array}$

$\begin{array}{lllll}0.4999220947197069 & 0.6122371150543516 & 0.1020705182383377\end{array}$

$\begin{array}{llll}0.3998462570018086 & 0.7787617996850967 & 0.1022190503346117\end{array}$

$\begin{array}{llll}0.5998402435118149 & 0.7789732234098489 & 0.1022739697646617\end{array}$

$\begin{array}{llll}0.6994939733054776 & 0.6119015490084262 & 0.1021474283688915\end{array}$

$\begin{array}{llll}0.2992229147932734 & 0.6115910534069837 & 0.1019016878140271\end{array}$

$\begin{array}{llll}0.1989100456703643 & 0.4440044482439971 & 0.1023116376223888\end{array}$

$\begin{array}{lllll}0.8004514154312987 & 0.4440324251778691 & 0.1020473707064917\end{array}$

0.39916985934290020 .44427716179057090 .1018049831099206

$\begin{array}{llll}0.5996204039488775 & 0.4447004898151996 & 0.1018562723519354\end{array}$

$\begin{array}{llll}0.9000497486702113 & 0.2776082598839613 & 0.1022806023554585\end{array}$

$\begin{array}{llll}0.0996490094446893 & 0.2777600050747887 & 0.1025847955125002\end{array}$

$\begin{array}{llll}0.4999223516538566 & 0.9449029512173043 & 0.1024414626660997\end{array}$

$0.8000718928407393 \quad 0.11109040992672980 .1023682754388222$

$\begin{array}{llll}0.1995188448853645 & 0.1108652921382138 & 0.1023178244913884\end{array}$

$\begin{array}{llll}-0.0003455610339390 & 0.1109063964922198 & 0.1022976573144504\end{array}$

$\begin{array}{llll}0.6999844575192050 & 0.9448533673758404 & 0.1023527963870349\end{array}$

$\begin{array}{llll}0.2997813809498747 & 0.9448285761146565 & 0.1023150406198677\end{array}$

$\begin{array}{llll}0.0995947485488608 & 0.9442649886018256 & 0.1022683973966828\end{array}$

$\begin{array}{llll}0.8998678247572061 & 0.9442848314398499 & 0.1023272410242980\end{array}$

$\begin{array}{llll}0.6002127724847084 & 0.1111021548594193 & 0.1022224603432613\end{array}$

$\begin{array}{llll}0.3997696415529059 & 0.1111093718543580 & 0.1021734202792386\end{array}$

$\begin{array}{llll}0.2986562963021667 & 0.2773234352888292 & 0.1021400168494600\end{array}$

$\begin{array}{llll}0.7005470944568425 & 0.2774627327014923 & 0.1021532108296264\end{array}$

$\begin{array}{llll}0.7999310477408917 & 0.7779842441941101 & 0.1022594164353479\end{array}$

$\begin{array}{llll}0.9994299256583806 & 0.4443331586885250 & 0.1022780488760730\end{array}$

$\begin{array}{llll}0.1993660098557589 & 0.7780654264341097 & 0.1022046729378651\end{array}$

$\begin{array}{llll}0.9996211991432354 & 0.7775257616794979 & 0.1023325074909532\end{array}$

$\begin{array}{llll}0.4999277461991208 & 0.2775485000859965 & 0.1019770926947078\end{array}$

$\begin{array}{llll}0.8997988165262417 & 0.6109266558408445 & 0.1022321669691362\end{array}$

$\begin{array}{llll}0.0991366100698935 & 0.6110465842648131 & 0.1022409920909310\end{array}$

$\begin{array}{llll}0.5995598293414378 & 0.6697927854984279 & 0.1536502849824228\end{array}$

$\begin{array}{llll}0.3968118086410024 & 0.6694156135412176 & 0.1528803849802348\end{array}$ 
$0.2971991469836597 \quad 0.50168581628028060 .1526348182459095$

$\begin{array}{llll}0.7000843324747285 & 0.5004027566992836 & 0.1526705997067343\end{array}$

$\begin{array}{llll}0.4980748966912711 & 0.5007168079009189 & 0.1522346625714791\end{array}$

$\begin{array}{llll}0.4992179546676340 & 0.8353658791942561 & 0.1544540345819331\end{array}$

$\begin{array}{llll}0.0994741182728852 & 0.1668533774988316 & 0.1544935292701530\end{array}$

$\begin{array}{llll}0.8995722307391694 & 0.1671090928492435 & 0.1545393646810247\end{array}$

$\begin{array}{llll}0.1993894416715223 & 0.0003316399310255 & 0.1545002113013452\end{array}$

$\begin{array}{lllll}0.7998828291235179 & 0.0005307725331892 & 0.1545298808110274\end{array}$

$\begin{array}{llll}-0.0003814069804815 & 0.0003127263889697 & 0.1544130897584409\end{array}$

$\begin{array}{llll}-0.0009966307514775 & 0.3338688114444523 & 0.1545311106087507\end{array}$

$\begin{array}{llll}0.7004127535606549 & 0.8341755062924840 & 0.1545451109251536\end{array}$

$\begin{array}{llll}0.2986222937355070 & 0.8345792678327816 & 0.1542997457440289\end{array}$

$\begin{array}{llll}0.6000772773280396 & 0.0009080329118830 & 0.1545963657841317\end{array}$

$\begin{array}{llll}0.3996288490862964 & 0.0008242939789531 & 0.1545482061008571\end{array}$

$\begin{array}{llll}0.2998290271680918 & 0.1662021993506489 & 0.1543849194440567\end{array}$

$\begin{array}{llll}0.6995467710263346 & 0.1673381304219534 & 0.1547835215654917\end{array}$

$\begin{array}{llll}0.1989584936410821 & 0.3335842481938277 & 0.1551977755997420\end{array}$

$\begin{array}{llll}0.7995355260188509 & 0.3337086363746625 & 0.1547809771397125\end{array}$

$\begin{array}{llll}0.8996642661549848 & 0.8335821647186652 & 0.1545471706646504\end{array}$

$\begin{array}{llll}0.0991250441475840 & 0.8338196453401796 & 0.1544171092018584\end{array}$

$\begin{array}{llll}0.4999914928340340 & 0.1663973395746816 & 0.1539568337612637\end{array}$

$\begin{array}{llll}0.3983658114650264 & 0.3317108690169335 & 0.1524466905946255\end{array}$

$\begin{array}{llll}0.6003604662442323 & 0.3335708148842488 & 0.1530761153931484\end{array}$

$\begin{array}{llll}0.0980980731467798 & 0.5007429454860653 & 0.1543830919153841\end{array}$

$0.79998536017417250 .6671032723744604 \quad 0.1541879192435384$

$\begin{array}{llll}0.8988297452327839 & 0.5002796595100064 & 0.1548057062456834\end{array}$

$\begin{array}{llll}0.1978305799537611 & 0.6677153535434424 & 0.1542923588676702\end{array}$

$\begin{array}{llll}0.9989090570840222 & 0.6668615741273697 & 0.1546793195928220\end{array}$

$\begin{array}{llll}0.3159340245023605 & 0.3814346518595043 & 0.2106545001414252\end{array}$

$\begin{array}{llll}0.5160282163424631 & 0.6953486669819814 & 0.2097521022230707\end{array}$

$\begin{array}{llll}0.4954786549908686 & 0.2720218333861359 & 0.2234074547148894\end{array}$

$\begin{array}{llll}0.6993134112118257 & 0.5912112159972002 & 0.2229907056434521\end{array}$

$\begin{array}{llll}0.4098700299539496 & 0.5422155525782200 & 0.2130619856897352\end{array}$

$\begin{array}{llll}0.5039493260544012 & 0.3762462822803922 & 0.2216847819726773\end{array}$

$\begin{array}{llll}0.4026923541530992 & 0.4328961243028845 & 0.2156840592232714\end{array}$

$\begin{array}{llll}0.6064164157059428 & 0.5370528135588813 & 0.2212328611519308\end{array}$

$\begin{array}{llll}0.6019048653486643 & 0.4289421967912473 & 0.2233316748247841\end{array}$

$\begin{array}{llll}0.5080663920808809 & 0.5973155645801594 & 0.2150747056671304\end{array}$

$\begin{array}{llll}0.3358896690437048 & 0.5865472000099469 & 0.2112155799387131\end{array}$

$\begin{array}{llll}0.6760661947178643 & 0.3852134636972884 & 0.2251798240863338\end{array}$

$\begin{array}{llll}0.6937282448188259 & 0.6667265383179591 & 0.2160453489284337\end{array}$

$\begin{array}{llll}0.7697082217701964 & 0.5561378566521723 & 0.2170507761620389\end{array}$

$\begin{array}{llll}0.5600524353968535 & 0.2277456173797831 & 0.2175841113784038\end{array}$

$\begin{array}{llll}0.4230311890828086 & 0.2429946088239831 & 0.2162149996543653\end{array}$

Zwitterion on Ag - VASP - revPBE+D3-calculated lattice - Preferred Epitaxy ( $\mathrm{T}$ - 30 site) Ag O N C H 
1.00000000000000

$\begin{array}{lll}14.5060000000000002 & 0.0000000000000000 & 0.0000000000000000\end{array}$

$\begin{array}{lll}0.0000000000000000 & 15.0760000000000005 & 0.0000000000000000\end{array}$

$\begin{array}{llll}0.0000000000000000 & 0.0000000000000000 & 40.0000000000000000\end{array}$

$\begin{array}{lllll}\mathrm{Ag} & \mathrm{O} & \mathrm{N} & \mathrm{C} & \mathrm{H}\end{array}$

$\begin{array}{lllll}120 & 2 & 2 & 6 & 6\end{array}$

Direct

$\begin{array}{llll}0.9987974951837949 & 0.3302903746185851 & 0.1810403859914681\end{array}$

$\begin{array}{llll}0.0001295993296473 & 0.6639639926874726 & 0.1810353440418833\end{array}$

$\begin{array}{llll}0.8995850846914140 & 0.1641681148699923 & 0.1810770197543508\end{array}$

$\begin{array}{lll}0.0989827882402483 & 0.1633035135060518 & 0.1809404111679010\end{array}$

$\begin{array}{llll}0.8997316005551099 & 0.4972298168277935 & 0.1813167387051024\end{array}$

0.69955962324058340 .16484100043209520 .1808934348457835

$\begin{array}{llll}0.9007220529254485 & 0.8310196921845017 & 0.1807705010455922\end{array}$

$\begin{array}{llll}0.1000860991816973 & 0.8304946620381962 & 0.1803855226299409\end{array}$

$\begin{array}{llll}0.8003173833929402 & 0.9982294454403497 & 0.1805517879417452\end{array}$

$\begin{array}{llll}0.0979828363360603 & 0.4966796519436636 & 0.1804044650442485\end{array}$

$\begin{array}{llll}0.1996886845953926 & 0.6638310807347194 & 0.1802801851038514\end{array}$

$\begin{array}{llll}0.9999580205227881 & 0.9972173860587656 & 0.1803731280306085\end{array}$

$\begin{array}{llll}0.1997527759702871 & 0.9970625385748811 & 0.1802754383059434\end{array}$

$\begin{array}{llll}0.7996550617288603 & 0.3306634602764969 & 0.1809119043771079\end{array}$

$\begin{array}{llll}0.3999688369275636 & 0.9972174413157495 & 0.1802498426488707\end{array}$

$\begin{array}{llll}0.6000618251735745 & 0.9974396226896471 & 0.1802197727947636\end{array}$

$\begin{array}{llll}0.2991606054286346 & 0.1629597241794496 & 0.1805396008718413\end{array}$

$\begin{array}{llll}0.2999868533378583 & 0.8304997834575161 & 0.1802413553537046\end{array}$

$\begin{array}{llll}0.7013945121237866 & 0.8321969293948174 & 0.1803041257431591\end{array}$

0.80152335911025120 .66445141098425190 .1805458164190742

$\begin{array}{llll}0.1979002267273394 & 0.3284219706327823 & 0.1804400173703491\end{array}$

$\begin{array}{llll}0.4999547099665427 & 0.8307653998809037 & 0.1802033692090242\end{array}$

$\begin{array}{llll}0.4999749477424767 & 0.1638298684922004 & 0.1804104969389780\end{array}$

$\begin{array}{llll}0.2956024656448391 & 0.4946803857226342 & 0.1804387165599036\end{array}$

$\begin{array}{llll}0.6030899068369410 & 0.6662439848317747 & 0.1800130125698754\end{array}$

$\begin{array}{llll}0.3997524690883738 & 0.6639598548119526 & 0.1785041834435405\end{array}$

$\begin{array}{llll}0.5996213063258254 & 0.3309222255191583 & 0.1794029988043213\end{array}$

$\begin{array}{llll}0.3982556209361294 & 0.3274731420140440 & 0.1782291712191730\end{array}$

$\begin{array}{llll}0.7020619042872950 & 0.4971592302818887 & 0.1779837264685649\end{array}$

$\begin{array}{llll}0.4998159687119050 & 0.4972169760519236 & 0.1757470532609012\end{array}$

$\begin{array}{llll}0.0998961855226869 & 0.6074811922342950 & 0.1198932774897566\end{array}$

$\begin{array}{llll}0.8001122527216723 & 0.1084974528181696 & 0.1199979460445354\end{array}$

$\begin{array}{llll}0.9999588356611389 & 0.7737990523097322 & 0.1198805299594752\end{array}$

$\begin{array}{llll}0.9998674297233185 & 0.1075383226149927 & 0.1199023498126857\end{array}$

$\begin{array}{llll}0.0993883589693444 & 0.2739752740728718 & 0.1198724609665231\end{array}$

$0.99986169464249990 .4405802440726636 \quad 0.1198899129376805$

$\begin{array}{llll}0.9000139442688421 & 0.6075316806443113 & 0.1199014429280588\end{array}$

$\begin{array}{llll}0.9000111439198620 & 0.2742542050676738 & 0.1199065646650427\end{array}$

$\begin{array}{llll}0.0999071184684945 & 0.9405349554869087 & 0.1197571187705756\end{array}$

$\begin{array}{llll}0.8003498032917257 & 0.7751233355009329 & 0.1198525193033517\end{array}$ 
$\begin{array}{lll}0.1996123671918198 & 0.1078241540703462 & 0.1198235079655820\end{array}$

0.90008996371240250 .94117501366594940 .1198352742376095

$\begin{array}{llll}0.2000572255581006 & 0.7742786759013748 & 0.1197373329691406\end{array}$

$\begin{array}{llll}0.2998114000656296 & 0.9418947845685617 & 0.1197511493267911\end{array}$

$\begin{array}{llll}0.7000772297281264 & 0.9422620049132862 & 0.1197546738854588\end{array}$

$\begin{array}{llll}0.3998967550971667 & 0.1087710103680138 & 0.1197707805910260\end{array}$

$\begin{array}{llll}0.6001386598752876 & 0.1089227988213332 & 0.1197906425364484\end{array}$

$\begin{array}{llll}0.5000169844196012 & 0.9424028084684708 & 0.1197426991246145\end{array}$

$\begin{array}{llll}0.2997989230319441 & 0.6069362375080242 & 0.1199151007565907\end{array}$

$\begin{array}{llll}0.1993260214651073 & 0.4407112739924297 & 0.1199127727691604\end{array}$

$\begin{array}{llll}0.6004019969108837 & 0.7758198658725767 & 0.1199356311583671\end{array}$

$\begin{array}{llll}0.3998977582108135 & 0.7757614376035041 & 0.1196336036398725\end{array}$

$\begin{array}{llll}0.7000059113265424 & 0.6092364982968111 & 0.1197253851056476\end{array}$

$\begin{array}{llll}0.7004883982759144 & 0.2749557991319938 & 0.1197247655685707\end{array}$

$\begin{array}{llll}0.8009766878621019 & 0.4410624119097837 & 0.1194882862345713\end{array}$

$\begin{array}{llll}0.2987639957995545 & 0.2741723086846306 & 0.1193623226343157\end{array}$

$\begin{array}{llll}0.5000471711087902 & 0.2753645674122602 & 0.1194152787117274\end{array}$

$\begin{array}{llll}0.3981139405426243 & 0.4414596072888461 & 0.1193792517151253\end{array}$

$\begin{array}{llll}0.5006255901425940 & 0.6103200072037470 & 0.1191560827291653\end{array}$

$\begin{array}{llll}0.6009880562469315 & 0.4418318364995699 & 0.1188430349362324\end{array}$

$\begin{array}{llll}0.0000000000000000 & 0.8888120000000015 & 0.0605819999999966\end{array}$

$\begin{array}{llll}0.1999939999999967 & 0.8888120000000015 & 0.0605819999999966\end{array}$

$\begin{array}{lll}0.3999870000000030 & 0.8888120000000015 & 0.0605819999999966\end{array}$

$\begin{array}{lll}0.0000000000000000 & 0.5555080000000032 & 0.0605819999999966\end{array}$

$\begin{array}{llll}0.1999939999999967 & 0.5555080000000032 & 0.0605819999999966\end{array}$

$\begin{array}{llll}0.0999970000000019 & 0.7221600000000024 & 0.0605819999999966\end{array}$

$\begin{array}{llll}0.0000000000000000 & 0.2222030000000004 & 0.0605819999999966\end{array}$

$\begin{array}{lll}0.5999809999999997 & 0.8888120000000015 & 0.0605819999999966\end{array}$

$\begin{array}{lll}0.7999739999999989 & 0.8888120000000015 & 0.0605819999999966\end{array}$

$\begin{array}{llll}0.3999870000000030 & 0.5555080000000032 & 0.0605819999999966\end{array}$

$\begin{array}{llll}0.5999809999999997 & 0.5555080000000032 & 0.0605819999999966\end{array}$

$\begin{array}{llll}0.4999839999999978 & 0.7221600000000024 & 0.0605819999999966\end{array}$

$\begin{array}{llll}0.1999939999999967 & 0.2222030000000004 & 0.0605819999999966\end{array}$

$\begin{array}{lllll}0.3999870000000030 & 0.2222030000000004 & 0.0605819999999966\end{array}$

$\begin{array}{llll}0.2999909999999986 & 0.3888549999999995 & 0.0605819999999966\end{array}$

$\begin{array}{lll}0.2999909999999986 & 0.7221600000000024 & 0.0605819999999966\end{array}$

$\begin{array}{llll}0.0999970000000019 & 0.0555510000000012 & 0.0605819999999966\end{array}$

0.09999700000000190 .38885499999999950 .0605819999999966

$\begin{array}{llll}0.7999739999999989 & 0.5555080000000032 & 0.0605819999999966\end{array}$

$\begin{array}{llll}0.8999710000000007 & 0.7221600000000024 & 0.0605819999999966\end{array}$

$\begin{array}{llll}0.5999809999999997 & 0.2222030000000004 & 0.0605819999999966\end{array}$

$\begin{array}{lll}0.7999739999999989 & 0.2222030000000004 & 0.0605819999999966\end{array}$

$\begin{array}{llll}0.6999769999999970 & 0.3888549999999995 & 0.0605819999999966\end{array}$

$\begin{array}{llll}0.6999769999999970 & 0.7221600000000024 & 0.0605819999999966\end{array}$

$\begin{array}{llll}0.4999839999999978 & 0.0555510000000012 & 0.0605819999999966\end{array}$

$\begin{array}{lllll}0.4999839999999978 & 0.3888549999999995 & 0.0605819999999966\end{array}$

$\begin{array}{llll}0.2999909999999986 & 0.0555510000000012 & 0.0605819999999966\end{array}$ 
$\begin{array}{llll}0.8999710000000007 & 0.0555510000000012 & 0.0605819999999966\end{array}$ $\begin{array}{llll}0.8999710000000007 & 0.3888549999999995 & 0.0605819999999966\end{array}$ $\begin{array}{llll}0.6999769999999970 & 0.0555510000000012 & 0.0605819999999966\end{array}$ $\begin{array}{lll}0.0000000000000000 & 0.6666090000000011 & 0.0000000000000000\end{array}$ 0.19999399999999670 .66660900000000110 .0000000000000000 $\begin{array}{lllll}0.3999870000000030 & 0.6666090000000011 & 0.0000000000000000\end{array}$ $\begin{array}{llll}0.2999909999999986 & 0.8332619999999977 & 0.0000000000000000\end{array}$ $\begin{array}{llll}0.0000000000000000 & 0.3333050000000028 & 0.0000000000000000\end{array}$ $\begin{array}{lll}0.1999939999999967 & 0.3333050000000028 & 0.0000000000000000\end{array}$ $\begin{array}{llll}0.0999970000000019 & 0.4999570000000020 & 0.0000000000000000\end{array}$ $\begin{array}{llll}0.0999970000000019 & 0.8332619999999977 & 0.0000000000000000\end{array}$ $0.0000000000000000 \quad 0.00000000000000000 .0000000000000000$ $\begin{array}{lll}0.5999809999999997 & 0.6666090000000011 & 0.0000000000000000\end{array}$ $\begin{array}{lll}0.7999739999999989 & 0.6666090000000011 & 0.0000000000000000\end{array}$ $\begin{array}{llll}0.6999769999999970 & 0.8332619999999977 & 0.0000000000000000\end{array}$ $\begin{array}{llll}0.3999870000000030 & 0.3333050000000028 & 0.0000000000000000\end{array}$ $\begin{array}{llll}0.5999809999999997 & 0.3333050000000028 & 0.0000000000000000\end{array}$ $\begin{array}{llll}0.4999839999999978 & 0.4999570000000020 & 0.0000000000000000\end{array}$ $\begin{array}{lllll}0.4999839999999978 & 0.8332619999999977 & 0.0000000000000000\end{array}$ $\begin{array}{llll}0.1999939999999967 & 0.0000000000000000 & 0.0000000000000000\end{array}$ $\begin{array}{llll}0.3999870000000030 & 0.0000000000000000 & 0.0000000000000000\end{array}$ $\begin{array}{lllll}0.2999909999999986 & 0.1666519999999991 & 0.0000000000000000\end{array}$ $\begin{array}{llll}0.2999909999999986 & 0.4999570000000020 & 0.0000000000000000\end{array}$ $\begin{array}{llll}0.0999970000000019 & 0.1666519999999991 & 0.0000000000000000\end{array}$ $\begin{array}{lll}0.7999739999999989 & 0.3333050000000028 & 0.0000000000000000\end{array}$ $\begin{array}{llll}0.8999710000000007 & 0.4999570000000020 & 0.0000000000000000\end{array}$ $\begin{array}{llll}0.8999710000000007 & 0.8332619999999977 & 0.0000000000000000\end{array}$ $\begin{array}{lll}0.5999809999999997 & 0.0000000000000000 & 0.0000000000000000\end{array}$ $\begin{array}{lll}0.7999739999999989 & 0.0000000000000000 & 0.0000000000000000\end{array}$ $\begin{array}{llll}0.6999769999999970 & 0.1666519999999991 & 0.0000000000000000\end{array}$ $\begin{array}{llll}0.6999769999999970 & 0.4999570000000020 & 0.0000000000000000\end{array}$ $\begin{array}{llll}0.4999839999999978 & 0.1666519999999991 & 0.0000000000000000\end{array}$ $\begin{array}{llll}0.8999710000000007 & 0.1666519999999991 & 0.0000000000000000\end{array}$ $\begin{array}{llll}0.3129600197940810 & 0.4907532493229073 & 0.2436150926253139\end{array}$ $\begin{array}{llll}0.5986357515048165 & 0.6523089359883188 & 0.2434052527000882\end{array}$ $\begin{array}{llll}0.6914339839051866 & 0.5002231676402550 & 0.2514258520685994\end{array}$ $\begin{array}{llll}0.4029214350639062 & 0.3393885516044519 & 0.2514019842669215\end{array}$ $\begin{array}{llll}0.5504830665180241 & 0.4148342719874373 & 0.2545608707016950\end{array}$ $\begin{array}{llll}0.5984256742368178 & 0.4955427670782485 & 0.2525599878527991\end{array}$ $\begin{array}{lllll}0.4541165065463614 & 0.4143247679220751 & 0.2525872578705145\end{array}$ $\begin{array}{llll}0.4004258752217534 & 0.4983599099154126 & 0.2482110900739343\end{array}$ $\begin{array}{llll}0.5493148805579132 & 0.5823632828291903 & 0.2481337438805734\end{array}$ $\begin{array}{llll}0.4512858050527726 & 0.5789991093037510 & 0.2472147787753266\end{array}$ $\begin{array}{llll}0.5883894647122568 & 0.3524169952914150 & 0.2565905738856857\end{array}$ $\begin{array}{llll}0.4303991905570871 & 0.2780548378227662 & 0.2540321287076399\end{array}$ $\begin{array}{llll}0.7321348964402695 & 0.4459182268505485 & 0.2535457580334937\end{array}$ $\begin{array}{llll}0.7202846463598802 & 0.5617033913773288 & 0.2502586866635707\end{array}$ 


\section{$\begin{array}{lll}0.3331670111861778 & 0.3472056060126492 & 0.2503693186807037\end{array}$ \\ $\begin{array}{llll}0.4132186186923569 & 0.6413598771565248 & 0.2456602563107458\end{array}$}

Zwitterion on $\mathrm{Au}$ - VASP - revPBE+D3-calculated lattice - Preferred Epitaxy ( $\underline{\mathrm{H}}_{\mathrm{hcp}}-60$ site) $\underline{\mathrm{Au} \mathrm{O} \mathrm{N} \mathrm{C} \mathrm{H}}$

1.00000000000000

$\begin{array}{lll}14.6319999999999997 & 0.0000000000000000 & 0.0000000000000000\end{array}$

$\begin{array}{lll}0.0000000000000000 & 15.2059999999999995 & 0.0000000000000000\end{array}$

$0.0000000000000000 \quad 0.0000000000000000 \quad 40.0000000000000000$

$\mathrm{Au} \quad \mathrm{O} \quad \mathrm{N} \quad \mathrm{C} \quad \mathrm{H}$

$\begin{array}{lllll}120 & 2 & 2 & 6 & 6\end{array}$

Direct

$0.0000000000000000 \quad 0.66660900000000110 .0000000000000000$

$\begin{array}{lll}0.0000000000000000 & 0.8888120000000015 & 0.0605819999999966\end{array}$

$\begin{array}{llll}0.1999939999999967 & 0.6666090000000011 & 0.0000000000000000\end{array}$

$\begin{array}{llll}0.3999870000000030 & 0.6666090000000011 & 0.0000000000000000\end{array}$

$\begin{array}{llll}0.2999909999999986 & 0.8332619999999977 & 0.0000000000000000\end{array}$

$\begin{array}{llll}0.1999939999999967 & 0.8888120000000015 & 0.0605819999999966\end{array}$

$\begin{array}{lll}0.3999870000000030 & 0.8888120000000015 & 0.0605819999999966\end{array}$

$\begin{array}{llll}0.0000000000000000 & 0.3333050000000028 & 0.0000000000000000\end{array}$

$\begin{array}{llll}0.1999939999999967 & 0.3333050000000028 & 0.0000000000000000\end{array}$

$\begin{array}{llll}0.0999970000000019 & 0.4999570000000020 & 0.0000000000000000\end{array}$

$\begin{array}{llll}0.0000000000000000 & 0.5555080000000032 & 0.0605819999999966\end{array}$

$\begin{array}{llll}0.1999939999999967 & 0.5555080000000032 & 0.0605819999999966\end{array}$

$\begin{array}{llll}0.0999970000000019 & 0.7221600000000024 & 0.0605819999999966\end{array}$

$\begin{array}{llll}0.9986557651360742 & 0.7772598406486725 & 0.1206436818003183\end{array}$

$\begin{array}{lllll}0.1996185754711897 & 0.7788035939154048 & 0.1198167343837943\end{array}$

$\begin{array}{llll}0.0999970000000019 & 0.8332619999999977 & 0.0000000000000000\end{array}$

$\begin{array}{llll}0.0990386173413185 & 0.9438167176039071 & 0.1200873792871705\end{array}$

$\begin{array}{llll}0.0000000000000000 & 0.0000000000000000 & 0.0000000000000000\end{array}$

$\begin{array}{llll}0.0000000000000000 & 0.2222030000000004 & 0.0605819999999966\end{array}$

$\begin{array}{llll}0.0010549825271116 & 0.4447178795270219 & 0.1205305701370557\end{array}$

0.00099158918714640 .66528715844211280 .1851561444413703

$\begin{array}{lll}0.5999809999999997 & 0.6666090000000011 & 0.0000000000000000\end{array}$

0.79997399999999890 .66660900000000110 .0000000000000000

$\begin{array}{lllll}0.6999769999999970 & 0.8332619999999977 & 0.0000000000000000\end{array}$

$\begin{array}{llll}0.5999809999999997 & 0.8888120000000015 & 0.0605819999999966\end{array}$

0.79997399999999890 .88881200000000150 .0605819999999966

$\begin{array}{llll}0.3999870000000030 & 0.3333050000000028 & 0.0000000000000000\end{array}$

$\begin{array}{llll}0.5999809999999997 & 0.3333050000000028 & 0.0000000000000000\end{array}$

$\begin{array}{llll}0.4999839999999978 & 0.4999570000000020 & 0.0000000000000000\end{array}$

$\begin{array}{llll}0.3999870000000030 & 0.5555080000000032 & 0.0605819999999966\end{array}$

$\begin{array}{llll}0.5999809999999997 & 0.5555080000000032 & 0.0605819999999966\end{array}$

$\begin{array}{llll}0.4999839999999978 & 0.7221600000000024 & 0.0605819999999966\end{array}$

$\begin{array}{llll}0.3999341196322133 & 0.7778075147618206 & 0.1215823173446995\end{array}$

$\begin{array}{lllll}0.5998926155949531 & 0.7795715729556655 & 0.1188675089905746\end{array}$

$\begin{array}{llll}0.4999839999999978 & 0.8332619999999977 & 0.0000000000000000\end{array}$ 
0.49846903043124620 .94749192253481730 .1197938320102054

$\begin{array}{llll}0.1999939999999967 & 0.0000000000000000 & 0.0000000000000000\end{array}$

$\begin{array}{llll}0.3999870000000030 & 0.0000000000000000 & 0.0000000000000000\end{array}$

$\begin{array}{llll}0.2999909999999986 & 0.1666519999999991 & 0.0000000000000000\end{array}$

$\begin{array}{lll}0.1999939999999967 & 0.2222030000000004 & 0.0605819999999966\end{array}$

$\begin{array}{llll}0.3999870000000030 & 0.2222030000000004 & 0.0605819999999966\end{array}$

$\begin{array}{llll}0.2999909999999986 & 0.3888549999999995 & 0.0605819999999966\end{array}$

$\begin{array}{llll}0.1977211910866756 & 0.4457067913597076 & 0.1199380634465112\end{array}$

$0.3974620716861299 \quad 0.4435948877595514 \quad 0.1190096784385471$

$\begin{array}{llll}0.2999909999999986 & 0.4999570000000020 & 0.0000000000000000\end{array}$

0.30049066727603450 .61232431145410690 .1217360010496466

0.20146904050365290 .66379289802218020 .1826721542451467

$\begin{array}{llll}0.4058278697111304 & 0.6606179422287094 & 0.1820910855495944\end{array}$

$\begin{array}{llll}0.2999909999999986 & 0.7221600000000024 & 0.0605819999999966\end{array}$

$\begin{array}{llll}0.3001250772129618 & 0.8306470167299953 & 0.1816991040981039\end{array}$

$\begin{array}{llll}0.3003078689389937 & 0.9459002708588140 & 0.1198345379382263\end{array}$

$\begin{array}{llll}0.0999970000000019 & 0.0555510000000012 & 0.0605819999999966\end{array}$

$\begin{array}{llll}0.9985090520750917 & 0.1079510685559723 & 0.1198357300393307\end{array}$

$\begin{array}{llll}0.2003576483729068 & 0.1114139349986191 & 0.1202059321416214\end{array}$

$\begin{array}{llll}0.0999970000000019 & 0.1666519999999991 & 0.0000000000000000\end{array}$

$\begin{array}{llll}0.0990948603373937 & 0.2773049943105659 & 0.1201254866599351\end{array}$

$\begin{array}{llll}0.9973512688782605 & 0.3348192929397140 & 0.1822570793197542\end{array}$

$\begin{array}{llll}0.1970970964473990 & 0.3276719130046805 & 0.1823737713970499\end{array}$

$\begin{array}{lll}0.0999970000000019 & 0.3888549999999995 & 0.0605819999999966\end{array}$

0.09745754876048730 .49965088285500240 .1828932290972775

$\begin{array}{llll}0.1005148617517964 & 0.6128346097571677 & 0.1204419438779518\end{array}$

$\begin{array}{llll}0.1007002589842590 & 0.8299322025677840 & 0.1827808316911822\end{array}$

$\begin{array}{llll}0.9995513466283871 & 0.9974793079618592 & 0.1817250773372460\end{array}$

$\begin{array}{lll}0.7999739999999989 & 0.3333050000000028 & 0.0000000000000000\end{array}$

$\begin{array}{llll}0.8999710000000007 & 0.4999570000000020 & 0.0000000000000000\end{array}$

$\begin{array}{llll}0.7999739999999989 & 0.5555080000000032 & 0.0605819999999966\end{array}$

$\begin{array}{llll}0.8999710000000007 & 0.7221600000000024 & 0.0605819999999966\end{array}$

$\begin{array}{llll}0.7983812100617519 & 0.7738493474733826 & 0.1205568439086879\end{array}$

$\begin{array}{llll}0.8999710000000007 & 0.8332619999999977 & 0.0000000000000000\end{array}$

$\begin{array}{llll}0.8968713238631270 & 0.9402034566666039 & 0.1203328246604879\end{array}$

$\begin{array}{llll}0.5999809999999997 & 0.0000000000000000 & 0.0000000000000000\end{array}$

$\begin{array}{llll}0.7999739999999989 & 0.0000000000000000 & 0.0000000000000000\end{array}$

$\begin{array}{llll}0.6999769999999970 & 0.1666519999999991 & 0.0000000000000000\end{array}$

$\begin{array}{lll}0.5999809999999997 & 0.2222030000000004 & 0.0605819999999966\end{array}$

$\begin{array}{llll}0.7999739999999989 & 0.2222030000000004 & 0.0605819999999966\end{array}$

$\begin{array}{llll}0.6999769999999970 & 0.3888549999999995 & 0.0605819999999966\end{array}$

0.60804450394845330 .44161269361500740 .1191999418564810

$\begin{array}{llll}0.8061617228219531 & 0.4412449012537086 & 0.1213837762156372\end{array}$

$\begin{array}{llll}0.6999769999999970 & 0.4999570000000020 & 0.0000000000000000\end{array}$

$\begin{array}{lllll}0.7062459488019026 & 0.6056166311187864 & 0.1194165616568341\end{array}$

$\begin{array}{llll}0.6071663974740281 & 0.6666297093081764 & 0.1762013173904502\end{array}$

$\begin{array}{llll}0.8034775154498602 & 0.6658722379036067 & 0.1851950637531846\end{array}$ 
$\begin{array}{lll}0.6999769999999970 & 0.7221600000000024 & 0.0605819999999966\end{array}$

$\begin{array}{llll}0.7047636694952412 & 0.8339690914740081 & 0.1828843831857601\end{array}$

$\begin{array}{llll}0.6984520210998659 & 0.9440690738361593 & 0.1203535291366058\end{array}$

$\begin{array}{llll}0.4999839999999978 & 0.0555510000000012 & 0.0605819999999966\end{array}$

$\begin{array}{llll}0.4009202826255302 & 0.1149997877236995 & 0.1205341650295985\end{array}$

$\begin{array}{llll}0.5998359911484896 & 0.1127258864220035 & 0.1204656005021771\end{array}$

$\begin{array}{llll}0.4999839999999978 & 0.1666519999999991 & 0.0000000000000000\end{array}$

$\begin{array}{llll}0.5028816159330219 & 0.2801317116678322 & 0.1202772558293625\end{array}$

$\begin{array}{llll}0.4000514317001939 & 0.3268782872727785 & 0.1819791365753057\end{array}$

$\begin{array}{llll}0.6012767942658489 & 0.3282988493049512 & 0.1841478777122091\end{array}$

$\begin{array}{llll}0.4999839999999978 & 0.3888549999999995 & 0.0605819999999966\end{array}$

$\begin{array}{llll}0.5031693807423869 & 0.4918067634963020 & 0.1758672886804449\end{array}$

0.49987656854406380 .61113774633654830 .1190620422817062

$\begin{array}{llll}0.5050961167762289 & 0.8383456542320751 & 0.1801213608151566\end{array}$

$\begin{array}{llll}0.1986046202586782 & 0.9974231925503794 & 0.1818014013814819\end{array}$

$\begin{array}{llll}0.3986668806823734 & 0.9989709760430668 & 0.1820696720660935\end{array}$

$\begin{array}{llll}0.2999909999999986 & 0.0555510000000012 & 0.0605819999999966\end{array}$

$\begin{array}{llll}0.2986269063468788 & 0.1631585359800950 & 0.1831630911660025\end{array}$

$\begin{array}{llll}0.2990756357571320 & 0.2782978950849978 & 0.1201786264668191\end{array}$

$\begin{array}{llll}0.2960588182146264 & 0.4904508576601443 & 0.1810166020831003\end{array}$

$\begin{array}{llll}0.0970718642363646 & 0.1643345836417998 & 0.1814810906495133\end{array}$

$\begin{array}{llll}0.8999710000000007 & 0.0555510000000012 & 0.0605819999999966\end{array}$

$\begin{array}{llll}0.7990277577832199 & 0.1079657998401659 & 0.1203716511811277\end{array}$

$\begin{array}{llll}0.8999710000000007 & 0.1666519999999991 & 0.0000000000000000\end{array}$

$\begin{array}{llll}0.9012196083246833 & 0.2749274021686526 & 0.1201904357141264\end{array}$

$\begin{array}{llll}0.7986922618727135 & 0.3347278875099710 & 0.1848942353450472\end{array}$

$\begin{array}{lllll}0.8999710000000007 & 0.3888549999999995 & 0.0605819999999966\end{array}$

$\begin{array}{llll}0.8981960877153812 & 0.5010223639839336 & 0.1857620829136178\end{array}$

$\begin{array}{llll}0.9031653922161945 & 0.6101389366127740 & 0.1211265325657706\end{array}$

$\begin{array}{llll}0.9019753804618196 & 0.8312197688674787 & 0.1841575481770872\end{array}$

$\begin{array}{llll}0.6038129181771138 & 0.0018241896541403 & 0.1828229159537302\end{array}$

$\begin{array}{llll}0.8024735593223231 & 0.9999380070861323 & 0.1832282153547448\end{array}$

$\begin{array}{llll}0.6999769999999970 & 0.0555510000000012 & 0.0605819999999966\end{array}$

$\begin{array}{lllll}0.7023493905621681 & 0.1665185831208339 & 0.1838662672927227\end{array}$

$\begin{array}{llll}0.7037627949520775 & 0.2755512228816819 & 0.1206705918446183\end{array}$

0.70037671882845390 .49916052296237540 .1847378481645622

$\begin{array}{llll}0.5007732564949565 & 0.1632100129996120 & 0.1836959801334726\end{array}$

$\begin{array}{llll}0.8993539481517953 & 0.1677877494633530 & 0.1821304015311098\end{array}$

$\begin{array}{llll}0.3372147649374562 & 0.5217103123757405 & 0.2524510248291074\end{array}$

$\begin{array}{llll}0.5028513725645455 & 0.7913227855804627 & 0.2513053876929683\end{array}$

$\begin{array}{llll}0.4920020421431832 & 0.4316728697447551 & 0.2545560958681076\end{array}$

$\begin{array}{llll}0.6588354565056680 & 0.7045822853591783 & 0.2558128701831280\end{array}$

$\begin{array}{llll}0.4119352557178326 & 0.6609334175366699 & 0.2463651944041110\end{array}$

$\begin{array}{llll}0.4998286153124677 & 0.5189735391217573 & 0.2548600907448998\end{array}$

$\begin{array}{llll}0.4083557270257060 & 0.5675161335280050 & 0.2517827225850815\end{array}$

$\begin{array}{lllll}0.5833131662534697 & 0.6556433990206837 & 0.2553625756839785\end{array}$

$\begin{array}{llll}0.5832482676300922 & 0.5636711081097476 & 0.2568474126699263\end{array}$ 
$\begin{array}{llll}0.4952674590392463 & 0.7091096241310023 & 0.2514084555373296\end{array}$

$\begin{array}{llll}0.3479203962340185 & 0.6975303059493535 & 0.2480251813901860\end{array}$

$\begin{array}{lllll}0.6474719330567450 & 0.5272913153564849 & 0.2584797622355746\end{array}$

$\begin{array}{lllll}0.6502762974637296 & 0.7704819274357940 & 0.2517466901006455\end{array}$

$\begin{array}{lllll}0.7221063557505335 & 0.6780236443717611 & 0.2531883586502596\end{array}$

$\begin{array}{lllll}0.5468534291074763 & 0.3917172985891748 & 0.2511374006343770\end{array}$

$\begin{array}{lllll}0.4279198806134640 & 0.4069994058300803 & 0.2505152983623469\end{array}$

Zwitterion on $\mathrm{Cu}-\mathrm{ADF}$ - revPBE+D3- T0 site

130

O $16.4707420 .96485 \quad 8.39774$

O $21.2887220 .78634 \quad 8.39717$

N 16.3542718 .282698 .88359

N $21.22601 \quad 18.12174 \quad 8.88909$

C $18.8836020 .96619 \quad 8.49848$

C $17.5818918 .87122 \quad 8.78646$

C $17.5961020 .34023 \quad 8.57766$

C $20.0377818 .78627 \quad 8.78908$

C $18.7864918 .13726 \quad 8.82829$

C $20.1212120 .24957 \quad 8.57827$

H 18.9210122 .058418 .46553

H $18.7482017 .04582 \quad 8.87274$

H 22.0519418 .669168 .63032

H 21.2677217 .132368 .64178

H $16.25646 \quad 17.295808 .64189$

H 15.5593618 .871678 .62171

Cu $11.27294 \quad 12.28901 \quad 4.09436$

$\begin{array}{llll}\mathrm{Cu} & 11.27100 & 22.41600 & 2.04500\end{array}$

Cu $11.27100 \quad 13.73900 \quad 2.04500$

$\begin{array}{llll}\mathrm{Cu} & 11.27100 & 18.07700 & 2.04500\end{array}$

$\mathrm{Cu} 11.2778316 .62603 \quad 4.09038$

$\mathrm{Cu} 11.27294 \quad 25.30501 \quad 4.09436$

$\mathrm{Cu} 12.5232018 .79712 \quad 4.09115$

Cu $12.5226923 .13549 \quad 4.09163$

Cu $11.2686520 .96967 \quad 4.10387$

Cu $11.27157 \quad 15.18710 \quad 6.18624$

Cu $11.27250 \quad 23.86492 \quad 6.17920$

Cu $11.28031 \quad 19.51507 \quad 6.18748$

Cu 12.5209621 .691136 .18502

$\mathrm{Cu} 18.78492 \quad 12.299104 .09085$

Cu $21.29315 \quad 12.29749 \quad 4.09152$

$\begin{array}{llll}\mathrm{Cu} & 16.27801 & 12.29718 \quad 4.09170\end{array}$

Cu $13.77392 \quad 12.28954 \quad 4.09421$

Cu $23.7969412 .28901 \quad 4.09436$

$\begin{array}{llll}\mathrm{Cu} & 12.52400 & 24.58500 & 2.04500\end{array}$

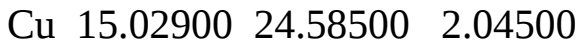


$\begin{array}{llll}\mathrm{Cu} & 17.53300 & 24.58500 & 2.04500\end{array}$

$\begin{array}{llll}\mathrm{Cu} & 12.52400 & 20.24600 & 2.04500\end{array}$

$\begin{array}{llll}\mathrm{Cu} & 15.02900 & 20.24600 & 2.04500\end{array}$

$\begin{array}{llll}\mathrm{Cu} & 13.77600 & 22.41600 & 2.04500\end{array}$

$\begin{array}{llll}\mathrm{Cu} & 12.52400 & 15.90800 & 2.04500\end{array}$

$\begin{array}{llll}\mathrm{Cu} & 20.03800 & 24.58500 & 2.04500\end{array}$

$\begin{array}{lll}\text { Cu } 22.54300 & 24.58500 & 2.04500\end{array}$

$\begin{array}{llll}\mathrm{Cu} & 17.53300 & 20.24600 & 2.04500\end{array}$

$\begin{array}{llll}\mathrm{Cu} & 20.03800 & 20.24600 & 2.04500\end{array}$

$\begin{array}{llll}\mathrm{Cu} & 18.78600 & 22.41600 & 2.04500\end{array}$

$\begin{array}{llll}\mathrm{Cu} & 15.02900 & 15.90800 & 2.04500\end{array}$

$\begin{array}{llll}\mathrm{Cu} & 17.53300 & 15.90800 & 2.04500\end{array}$

$\begin{array}{llll}\mathrm{Cu} & 16.28100 & 18.07700 & 2.04500\end{array}$

$\begin{array}{llll}\mathrm{Cu} & 16.28100 & 22.41600 & 2.04500\end{array}$

$\begin{array}{llll}\mathrm{Cu} & 13.77600 & 13.73900 & 2.04500\end{array}$

$\begin{array}{llll}\mathrm{Cu} & 13.77600 & 18.07700 & 2.04500\end{array}$

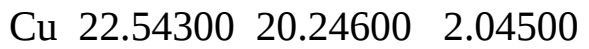

$\begin{array}{llll}\mathrm{Cu} & 23.79500 & 22.41600 & 2.04500\end{array}$

$\begin{array}{llll}\mathrm{Cu} & 20.03800 & 15.90800 & 2.04500\end{array}$

$\begin{array}{llll}\mathrm{Cu} & 22.54300 & 15.90800 & 2.04500\end{array}$

$\begin{array}{llll}\mathrm{Cu} & 21.29100 & 18.07700 \quad 2.04500\end{array}$

$\begin{array}{llll}\mathrm{Cu} & 21.29100 & 22.41600 & 2.04500\end{array}$

$\begin{array}{llll}\mathrm{Cu} & 18.78600 & 13.73900 & 2.04500\end{array}$

Cu $18.78600 \quad 18.07700 \quad 2.04500$

$\begin{array}{llll}\mathrm{Cu} & 16.28100 & 13.73900 & 2.04500\end{array}$

$\begin{array}{llll}\mathrm{Cu} & 23.79500 & 13.73900 \quad 2.04500\end{array}$

$\begin{array}{llll}\mathrm{Cu} & 23.79500 & 18.07700 & 2.04500\end{array}$

$\begin{array}{llll}\mathrm{Cu} & 21.29100 & 13.73900 & 2.04500\end{array}$

Cu $18.7793520 .97920 \quad 4.07037$

$\begin{array}{llll}\mathrm{Cu} & 17.52967 & 23.14735 & 4.09050\end{array}$

Cu $20.0390023 .15072 \quad 4.08426$

$\begin{array}{llll}\mathrm{Cu} & 21.29701 & 20.97892 \quad 4.08422\end{array}$

$\mathrm{Cu} 16.2726320 .97951 \quad 4.08236$

$\begin{array}{llll}\mathrm{Cu} & 15.01236 & 18.79479 & 4.07291\end{array}$

Cu $22.55752 \quad 18.79693 \quad 4.07809$

$\mathrm{Cu} 17.52632 \quad 18.79976 \quad 4.06667$

Cu $20.0474918 .80212 \quad 4.07093$

Cu $23.8018316 .62603 \quad 4.09038$

Cu $13.76966 \quad 16.62567 \quad 4.09055$

Cu $18.78492 \quad 25.31510 \quad 4.09085$

Cu $22.54571 \quad 14.45955 \quad 4.09539$

$\begin{array}{llll}\mathrm{Cu} & 15.02600 & 14.45936 & 4.09515\end{array}$

$\mathrm{Cu} 12.52392 \quad 14.458204 .09681$

Cu $21.29315 \quad 25.31349 \quad 4.09152$

Cu $16.2780125 .31318 \quad 4.09170$

$\mathrm{Cu} 13.77392 \quad 25.30554 \quad 4.09421$

Cu $23.7969425 .30501 \quad 4.09436$ 
Cu $20.0399514 .45799 \quad 4.09266$

$\mathrm{Cu} 17.53197 \quad 14.45830 \quad 4.09267$

$\mathrm{Cu} 16.2714816 .62103 \quad 4.07966$

Cu $21.3003616 .62144 \quad 4.07922$

Cu $22.5463723 .13590 \quad 4.10370$

Cu 25.0472018 .797124 .09115

Cu $15.0236123 .13748 \quad 4.10161$

Cu 25.04669 23.13549 4.09163

Cu $18.7863016 .61967 \quad 4.06470$

Cu $23.7926520 .96967 \quad 4.10387$

Cu 13.7744420 .968354 .10046

Cu $20.0246821 .71707 \quad 6.10368$

Cu 17.5160721 .713146 .13435

$\mathrm{Cu} 16.2512719 .511556 .07673$

Cu 21.3186519 .520726 .09506

Cu 18.7831619 .517316 .07323

Cu $18.7815623 .88060 \quad 6.16804$

$\mathrm{Cu} 13.7755615 .18715 \quad 6.18618$

Cu $23.79557 \quad 15.18710 \quad 6.18624$

Cu 15.0265213 .020716 .18128

Cu $22.5438013 .02000 \quad 6.18114$

Cu 12.5232813 .018386 .18151

Cu $12.5236017 .35325 \quad 6.19415$

Cu 21.28788 23.872626 .17091

Cu 16.2769923 .875226 .17217

Cu 20.0382713 .021546 .18056

Cu $17.53247 \quad 13.023296 .17988$

Cu 16.2813015 .190036 .19232

Cu 21.2903115 .189646 .19187

Cu $15.0231517 .34775 \quad 6.16296$

Cu 22.5482517 .348086 .16035

Cu 23.7965023 .864926 .17920

Cu $13.7703623 .86537 \quad 6.17887$

Cu $18.78677 \quad 15.18555 \quad 6.18106$

Cu $17.5347917 .33649 \quad 6.09981$

Cu 20.0371817 .339026 .09829

$\mathrm{Cu} 13.7689219 .514716 .18784$

Cu $22.5413521 .69560 \quad 6.22046$

Cu $23.8043119 .51507 \quad 6.18748$

Cu $15.0157321 .69190 \quad 6.20497$

Cu 25.0449621 .691136 .18502

Cu 25.0480024 .585002 .04500

Cu $25.0480020 .24600 \quad 2.04500$

Cu 25.0480015 .908002 .04500

Cu $25.0479214 .45820 \quad 4.09681$

Cu 25.0472813 .018386 .18151

$\mathrm{Cu} 25.0476017 .35325 \quad 6.19415 \mathrm{i}$ 
Zwitterion on $\mathrm{Cu}$ - ADF - revPBE+D3- T30 site

130

O 16.0825219 .504328 .32458

O $20.3022621 .82541 \quad 8.34623$

N $17.3547017 .12187 \quad 8.39862$

N 21.6265319 .467718 .39763

C $18.15003 \quad 20.738438 .53810$

C 18.1079518 .284258 .66327

C $17.3837419 .53480 \quad 8.54317$

C $20.2444619 .45477 \quad 8.66811$

C $19.51157 \quad 18.25580 \quad 8.73740$

C $19.5792020 .73998 \quad 8.55211$

H $17.6251021 .69863 \quad 8.52714$

H $20.0354417 .29522 \quad 8.73308$

H $22.0773420 .35147 \quad 8.63697$

H 22.1640318 .645078 .65778

H $17.7514216 .23078 \quad 8.68403$

H 16.3649917 .224248 .62648

Cu $10.0190015 .90800 \quad 2.04500$

$\mathrm{Cu} 11.2710018 .07700 \quad 2.04500$

Cu $10.0255618 .79595 \quad 4.08049$

$\mathrm{Cu} 11.2769316 .622114 .07947$

$\mathrm{Cu} \quad 8.7700016 .62319 \quad 4.07412$

Cu $12.52531 \quad 18.793394 .08058$

Cu $12.5249023 .13675 \quad 4.08867$

Cu 11.2717320 .966834 .08079

Cu $11.2731315 .17860 \quad 6.18137$

$\mathrm{Cu} 10.0309417 .322956 .14076$

Cu 11.2969919 .519106 .15270

Cu 12.5274121 .698026 .18125

Cu 17.5330011 .569002 .04500

Cu $20.0380011 .56900 \quad 2.04500$

$\mathrm{Cu} 20.0421110 .122754 .10155$

Cu 18.7859612 .294014 .08914

Cu 21.2902012 .296834 .08880

$\mathrm{Cu} 16.2794312 .290864 .09048$

$\mathrm{Cu} 18.7819010 .862756 .16818$

Cu $15.0290024 .58500 \quad 2.04500$

Cu $17.5330024 .58500 \quad 2.04500$

Cu $12.5240020 .24600 \quad 2.04500$

Cu $15.0290020 .24600 \quad 2.04500$

Cu $13.7760022 .41600 \quad 2.04500$

Cu $12.5240015 .90800 \quad 2.04500$

Cu 20.0380024 .585002 .04500

Cu $22.5430024 .58500 \quad 2.04500$

Cu $17.5330020 .24600 \quad 2.04500$ 
$\begin{array}{lll}\text { Cu } 20.03800 & 20.24600 & 2.04500\end{array}$

Cu 18.7860022 .416002 .04500

Cu 15.0290015 .908002 .04500

$\begin{array}{llll}\mathrm{Cu} & 17.53300 & 15.90800 & 2.04500\end{array}$

$\begin{array}{llll}\mathrm{Cu} & 16.28100 & 18.07700 & 2.04500\end{array}$

$\begin{array}{llll}\mathrm{Cu} & 16.28100 & 22.41600 & 2.04500\end{array}$

$\begin{array}{llll}\mathrm{Cu} & 13.77600 & 13.73900 & 2.04500\end{array}$

Cu $13.7760018 .07700 \quad 2.04500$

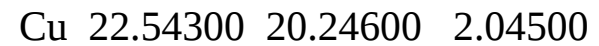

Cu $23.7950022 .41600 \quad 2.04500$

Cu $20.0380015 .90800 \quad 2.04500$

Cu $22.5430015 .90800 \quad 2.04500$

Cu $21.2910018 .07700 \quad 2.04500$

$\begin{array}{llll}\mathrm{Cu} & 21.29100 & 22.41600 & 2.04500\end{array}$

$\begin{array}{llll}\mathrm{Cu} & 18.78600 & 13.73900 & 2.04500\end{array}$

Cu $18.7860018 .07700 \quad 2.04500$

Cu 16.2810013 .739002 .04500

$\begin{array}{llll}\mathrm{Cu} & 23.79500 & 18.07700 & 2.04500\end{array}$

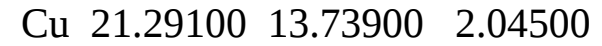

Cu $18.8018020 .98370 \quad 4.08828$

$\mathrm{Cu} 17.53523 \quad 23.14183 \quad 4.08683$

Cu $20.0421123 .13875 \quad 4.10155$

Cu $21.2903120 .96842 \quad 4.11364$

Cu $16.2781420 .95584 \quad 4.10082$

Cu $15.0243518 .80000 \quad 4.09276$

Cu 22.5495618 .795954 .08049

Cu 17.5118418 .789934 .11366

Cu $20.05397 \quad 18.80651 \quad 4.08850$

Cu 23.8009316 .622114 .07947

Cu 13.7698516 .621314 .08301

Cu $18.78596 \quad 25.310014 .08914$

Cu $22.5452514 .45785 \quad 4.09073$

$\begin{array}{llll}\mathrm{Cu} & 15.02134 & 14.45390 & 4.08617\end{array}$

Cu $12.52044 \quad 14.45496 \quad 4.09070$

Cu 21.2902025 .312834 .08880

Cu $16.27943 \quad 25.306864 .09048$

Cu $13.7757725 .30395 \quad 4.09096$

Cu 20.0408114 .459824 .08436

Cu $17.53107 \quad 14.45588 \quad 4.08604$

Cu 16.2748316 .623964 .08554

Cu 21.2940016 .623194 .07412

Cu $22.5456623 .14174 \quad 4.08610$

Cu 25.0493118 .793394 .08058

$\begin{array}{llll}\mathrm{Cu} & 15.02713 & 23.13653 & 4.08999\end{array}$

Cu 25.0489023 .136754 .08867

$\mathrm{Cu} 18.7702516 .62990 \quad 4.08773$

Cu $23.7957320 .96683 \quad 4.08079$ 
Cu $13.7757220 .96835 \quad 4.08896$

Cu $20.09622 \quad 21.74538 \quad 6.20810$

Cu $17.5325921 .70659 \quad 6.10909$

$\mathrm{Cu} 16.19697 \quad 19.51073 \quad 6.21204$

Cu 21.3629919 .503106 .17562

Cu $18.78391 \quad 19.52621 \quad 6.05806$

Cu $18.78190 \quad 23.87875 \quad 6.16818$

$\mathrm{Cu} 13.76561 \quad 15.17350 \quad 6.18215$

Cu $15.02276 \quad 13.00669 \quad 6.18359$

$\begin{array}{llll}\mathrm{Cu} & 12.51110 & 17.34431 & 6.17689\end{array}$

Cu 21.31453 23.89801 6.15880

$\begin{array}{llll}\mathrm{Cu} & 16.28062 & 23.86326 & 6.17405\end{array}$

Cu $20.03883 \quad 13.01885 \quad 6.17710$

$\begin{array}{llll}\mathrm{Cu} & 17.53243 & 13.01325 & 6.18168\end{array}$

$\begin{array}{llll}\mathrm{Cu} & 16.26082 & 15.14717 & 6.15323\end{array}$

Cu $21.28892 \quad 15.18484 \quad 6.18204$

$\begin{array}{llll}\mathrm{Cu} & 14.98622 & 17.32670 & 6.13396\end{array}$

Cu $22.55494 \quad 17.32295 \quad 6.14076$

Cu $23.8079123 .87300 \quad 6.18111$

$\begin{array}{llll}\mathrm{Cu} & 13.77422 & 23.86623 & 6.17567\end{array}$

Cu $18.7928915 .15838 \quad 6.15542$

$\begin{array}{llll}\mathrm{Cu} & 17.49423 & 17.28429 & 6.17923\end{array}$

Cu $20.0425517 .33306 \quad 6.09586$

$\begin{array}{llll}\mathrm{Cu} & 13.73754 & 19.52292 & 6.16462\end{array}$

Cu $22.5877721 .71236 \quad 6.14875$

Cu 23.8209919 .519106 .15270

Cu $15.0149821 .70934 \quad 6.16998$

$\begin{array}{llll}\mathrm{Cu} & 25.05141 & 21.69802 & 6.18125\end{array}$

$\begin{array}{llll}\mathrm{Cu} & 18.78600 & 26.75500 & 2.04500\end{array}$

$\begin{array}{llll}\mathrm{Cu} & 16.28100 & 26.75500 & 2.04500\end{array}$

$\begin{array}{llll}\mathrm{Cu} & 15.02134 & 27.46990 & 4.08617\end{array}$

$\begin{array}{llll}\mathrm{Cu} & 17.53107 & 27.47188 & 4.08604\end{array}$

$\begin{array}{llll}\mathrm{Cu} & 15.02276 & 26.02269 & 6.18359\end{array}$

Cu $20.0388326 .03485 \quad 6.17710$

$\begin{array}{llll}\mathrm{Cu} & 17.53243 & 26.02925 & 6.18168\end{array}$

$\begin{array}{llll}\mathrm{Cu} & 16.26082 & 28.16317 & 6.15323\end{array}$

$\begin{array}{lll}\mathrm{Cu} & 25.04800 & 20.24600 \\ 2.04500\end{array}$

$\begin{array}{lll}\mathrm{Cu} & 26.30000 & 22.41600 \quad 2.04500\end{array}$

Cu $26.29972 \quad 20.96835 \quad 4.08896$ 